\title{
The Surgical Infection Society Revised Guidelines on the Management of Intra-Abdominal Infection
}

\author{
John E. Mazuski, ${ }^{1}$ Jeffrey M. Tessier, ${ }^{2}$ Addison K. May, ${ }^{3}$ Robert G. Sawyer, Evan P. Nadler, \\ Matthew R. Rosengart, ${ }^{6}$ Phillip K. Chang, ${ }^{7}$ Patrick J. O'Neill, ${ }^{8}$ Kevin P. Mollen, \\ Jared M. Huston, ${ }^{10}$ Jose J. Diaz, Jr, ${ }^{11}$ and Jose M. Prince ${ }^{12}$
}

\begin{abstract}
Background: Previous evidence-based guidelines on the management of intra-abdominal infection (IAI) were published by the Surgical Infection Society (SIS) in 1992, 2002, and 2010. At the time the most recent guideline was released, the plan was to update the guideline every five years to ensure the timeliness and appropriateness of the recommendations.

Methods: Based on the previous guidelines, the task force outlined a number of topics related to the treatment of patients with IAI and then developed key questions on these various topics. All questions were approached using general and specific literature searches, focusing on articles and other information published since 2008. These publications and additional materials published before 2008 were reviewed by the task force as a whole or by individual subgroups as to relevance to individual questions. Recommendations were developed by a process of iterative consensus, with all task force members voting to accept or reject each recommendation. Grading was based on the GRADE (Grades of Recommendation Assessment, Development, and Evaluation) system; the quality of the evidence was graded as high, moderate, or weak, and the strength of the recommendation was graded as strong or weak. Review of the document was performed by members of the SIS who were not on the task force. After responses were made to all critiques, the document was approved as an official guideline of the SIS by the Executive Council.

Results: This guideline summarizes the current recommendations developed by the task force on the treatment of patients who have IAI. Evidence-based recommendations have been made regarding risk assessment in individual patients; source control; the timing, selection, and duration of antimicrobial therapy; and suggested approaches to patients who fail initial therapy. Additional recommendations related to the treatment of pediatric patients with IAI have been included.

Summary: The current recommendations of the SIS regarding the treatment of patients with IAI are provided in this guideline.
\end{abstract}

\footnotetext{
${ }^{1}$ Department of Surgery, Washington University School of Medicine, Saint Louis, Missouri.

${ }^{2}$ JPS Health System, Fort Worth, Texas.

${ }^{3}$ Department of Surgery, Vanderbilt University, Nashville, Tennessee.

${ }^{4}$ Department of Surgery, University of Virginia, Charlottesville, Virginia.

${ }^{5}$ Division of Pediatric Surgery, Children's National Medical Center, Washington, DC.

${ }^{6}$ Department of Surgery, University of Pittsburgh, Pittsburgh, Pennsylvania.

${ }^{7}$ Department of Surgery, University of Kentucky, Lexington, Kentucky.

${ }^{8}$ Trauma Department, Abrazo West Campus, Goodyear, Arizona.

${ }^{9}$ Division of Pediatric Surgery, Department of Surgery, University of Pittsburgh, Pittsburgh, Pennsylvania.

${ }^{10}$ Department of Surgery, Hofstra Northwell School of Medicine, Hempstead, New York.

${ }^{11}$ Department of Surgery, University of Maryland School of Medicine, Baltimore, Maryland.

${ }^{12}$ Departments of Surgery and Pediatrics, Hofstra-Northwell School of Medicine, Hempstead, New York.

This document is available on the Surgical Infection Society website, www.sisna.org.

(C) John E. Mazuski, et al., 2016; Published by Mary Ann Liebert, Inc. This Open Access article is distributed under the terms of the Creative Commons Attribution Noncommercial License (http://creativecommons.org/licenses/by-nc/4.0/) which permits any noncommercial use, distribution, and reproduction in any medium, provided the original author(s) and the source are credited.
} 


\section{EXECUTIVE SUMMARY}

Intra-abdominal infection (IAI) is a common disease process managed by surgical practitioners. The Surgical Infection Society (SIS) developed and disseminated guidelines for the management of these infections in 1992 [1], in 2002 [2,3], and most recently in 2010 as a joint guideline with the Infectious Diseases Society of America (IDSA) [4]. Since the 2010 guideline, additional challenges have arisen in the management of these infections, in part because of the aging of the population and the burden of chronic disease in these patients, and in part because of the increased prevalence of resistant bacteria and fungi in both the healthcare setting and the community. Nonetheless, advances in the management of these infections have also been made; newer approaches to source control are now available, as are new antibiotic agents that may meet some of the challenges posed by resistant pathogens.

To maintain the clinical relevance of the guideline, the SIS appointed a task force to revise the 2010 guideline. This task force included members of the Therapeutics and Guidelines Committee as well as additional individuals from the SIS with expertise in the subject matter. The task force selected subjects from the previous guideline for updating, developed specific questions for review, and then used the best available contemporary evidence to formulate recommendations. The task force evaluated the quality of the evidence and the strength of the recommendations using the GRADE (Grades of Recommendation Assessment, Development, and Evaluation) nomenclature, which has now become widely accepted as a standard for guidelines. Once completed, the entire document was subjected to external review by additional experts from the SIS, modified according to these reviews by consensus of the task force and sent to the Executive Council of the SIS for final approval.

All judgments regarding interpretation of this evidence and the GRADE assignments were exercised by the members of the task force and subsequent reviewers based on their individual and collective expertise, recognizing that the evidence could be interpreted differently by others. As with previous guidelines, these recommendations were designed to support clinicians in making appropriate treatment decisions and not designed to supplant the judgment of the individual practitioner [4].

\section{Risk assessment}

Assessing the risk of an adverse outcome in patients with IAI is important in optimizing selection of source control and antimicrobial therapy. Specific recommendations include:

- Use phenotypic and physiologic factors, including signs of sepsis or septic shock, extremes of age, and patient co-morbidities; the extent of abdominal infection and adequacy of initial source control; and the presence or persistence of resistant or opportunistic pathogens in assessing risk for treatment failure and mortality in patients with IAI (Grade 1-B).

- Characterize patients as being at either lower or higher risk for treatment failure or death, and as having either a community-acquired IAI (CA-IAI) or a healthcare or hospital-associated IAI (HA-IAI), including a postoperative infection, for purposes of planning source control and empiric antimicrobial therapy (Grade 2-C).
- Identify patients with IAI meeting Surviving Sepsis Campaign criteria for sepsis or septic shock and those having an Acute Physiology and Chronic Health Evaluation II score greater than or equal to 10 as higher-risk patients (Grade 1-B). Consider patients with at least two physiologic/phenotypic risk factors for an adverse outcome, those having diffuse peritonitis, and those having delayed or inadequate source control as potential higher-risk patients (Grade 2-B).

- Identify patients who have been hospitalized for at least 48 hours during the previous 90 days; those residing in a skilled nursing or long-term care facility during the previous 30 days; those who have received intravenous (IV) infusion therapy, wound care, or renal replacement therapy within the preceding 30 days; those who have received several days of broad-spectrum antimicrobial therapy within the previous 90 days; those who have postoperative infections; and those known to have been colonized or infected previously with a resistant pathogen as having HA-IAI and at potential risk for infection because of resistant or opportunistic organisms (Grade 2-B).

\section{Source control}

Source control is considered fundamental to the treatment of most patients with IAI. Specific recommendations regarding source control include:

- Routinely use a source control procedure to remove infected fluid and tissue and to prevent ongoing contamination in patients with IAI except for those patients with clinical problems for which there is clear evidence that a non-interventional approach is associated with a good clinical outcome (Grade 1-A).

- Undertake source control within 24 hours of the diagnosis of IAI, except for those infections for which clinical evidence indicates a non-interventional or delayed approach is appropriate (Grade 2-B). Undertake source control in a more urgent manner in patients with sepsis or septic shock (Grade 2-C).

- Use the least invasive approach that is able to achieve adequate source control, at least on a temporary basis, in patients with IAI (Grade 1-B).

- Consider use of alternative or temporizing approaches to source control in patients with major physiologic instability, those with diffuse infections, and those with ongoing bowel ischemia who are considered at higher risk for initial source control failure (Grade 2-B).

- Use abbreviated laparotomy and temporary abdominal closure techniques in critically ill patients with IAI if closure of the abdomen would create meaningful intraabdominal hypertension, if the patient's physiologic reserves are severely compromised, if there is an inability to achieve adequate source control with the initial procedure, or if there is a plan for a second look laparotomy because of mesenteric ischemia (Grade 1-B).

- Do not use routine planned re-laparotomy in higher-risk patients with severe peritonitis when adequate source control can be obtained at the time of the index procedure; treat such patients with on-demand rather than scheduled re-laparotomy (Grade 1-B).

- Irrigate with crystalloid fluid to remove visible debris and gross contamination before abdominal closure in patients 
undergoing laparotomy for IAI, generally limiting lavage to those areas with gross involvement (Grade 2-B).

\section{Microbiologic evaluation}

Microbiologic evaluation may be useful in selected patients, but is not necessary in most. Specific recommendations include:

- Do not routinely obtain peritoneal fluid cultures in lower-risk patients with CA-IAI for purposes of guiding antimicrobial therapy (Grade 1-B).

- Obtain cultures of peritoneal fluid or infected tissue in higher-risk patients with CA-IAI and in patients with HA-IAI to identify potential resistant or opportunistic pathogens (Grade 1-C).

- Consider obtaining cultures in all patients with IAI for epidemiologic purposes if adequate resources are available to aggregate and analyze the data and the information can be used to guide empiric antimicrobial therapy (Grade 2-C).

\section{Intravenous antimicrobial agents}

Many IV antimicrobial agents are potentially useful in the treatment of patients with IAI, as a supplement to source control. Specific recommendations regarding antimicrobial agents for the management of IAI include:

\section{A. General principles}

- Use antimicrobial regimens that have activity against the typical gram-negative Enterobacteriaceae, grampositive cocci, and obligate anaerobes involved in these infections (Grade 1-A).

\section{B. Aminoglycoside-based regimens}

- Do not use aminoglycoside-based regimens routinely for empiric therapy (Grade 1-B). Consider use of these agents for treatment of neonatal patients and for management of IAI because of resistant gram-negative organisms in all patients, if other agents are not suitable (Grade 2-B).

\section{Penicillin- $\beta$-lactamase inhibitor combinations}

- Do not use ampicillin-sulbactam routinely for empiric therapy (Grade 2-B).

- Do not use IV amoxicillin-clavulanic acid routinely for empiric therapy (Grade 2-B).

- Consider use of ticarcillin-clavulanic acid as an option for empiric therapy of lower-risk adults and children, if this agent again becomes available (Grade 2-B).

- Use piperacillin-tazobactam for empiric therapy of adults and children (Grade 1-A), but reserve this agent primarily for higher-risk patients because of its broader-spectrum antimicrobial activity (Grade 2-C).

D. Cephalosporin-based regimens and cephalosporin- $\beta$ lactamase inhibitor combinations

- Do not use cefoxitin and cefotetan routinely for empiric therapy (Grade 2-B).

- Do not use cefazolin plus metronidazole routinely for empiric therapy (Grade 2-C).
- Consider use of cefuroxime plus metronidazole as an option for empiric therapy of lower-risk adults and children (Grade 2-B).

- Use cefotaxime or ceftriaxone plus metronidazole for empiric therapy of lower-risk adults and children (Grade 1-A).

- Consider use of ceftazidime plus metronidazole as an option for empiric therapy of adults and children (Grade 2-A), but reserve this regimen primarily for higher-risk patients because of its broader-spectrum antimicrobial activity (Grade 2-C).

- Use cefepime plus metronidazole for empiric therapy of adults and children (Grade 1-A), but reserve this regimen primarily for higher-risk patients because of its broader-spectrum antimicrobial activity (Grade 2-C).

- Consider use of cefoperazone-sulbactam as an option for empiric therapy of lower-risk adults and children, in areas where this agent is available (Grade 2-B).

- Consider use of ceftolozane-tazobactam plus metronidazole as an option for empiric therapy of adults (Grade 2-A), but reserve this regimen primarily for higher-risk patients strongly suspected or proven to be infected with resistant strains of Pseudomonas aeruginosa, for which other agents are not suitable (Grade 2-C).

- Consider use of ceftazidime-avibactam plus metronidazole as an option for empiric therapy of adults (Grade 2-A), but reserve this regimen primarily for higher-risk patients strongly suspected or proven to be infected with Klebsiella pneumoniae carbapenemase (KPC)producing Enterobacteriaceae, for which other agents are not suitable (Grade 2-C).

\section{E. Aztreonam-based regimen}

- Consider use of aztreonam plus metronidazole plus vancomycin as an option for empiric therapy of adults and children (Grade 2-B), but reserve this regimen primarily for higher-risk patients, particularly those with serious $\beta$-lactam allergies, because of its broaderspectrum activity (Grade, 2-C).

\section{F. Carbapenems}

- Use ertapenem for empiric therapy of lower-risk adults and children (Grade 1-A).

- Use doripenem for empiric therapy of adults (Grade 1A), but reserve this agent primarily for higher-risk patients because of its broader-spectrum antimicrobial activity (Grade 2-C). Do not use doripenem for empiric therapy of children unless no other options are available (Grade 1-C).

- Use imipenem-cilastatin or meropenem for the empiric therapy of adults and children (Grade 1-A), but reserve these agents primarily for higher-risk patients because of their broader-spectrum antimicrobial activity (Grade 2-C).

\section{G. Fluoroquinolones and fluoroquinolone-based regimens}

- Use moxifloxacin for empiric therapy of lower-risk adults, but use with caution in areas where there is a high incidence of fluoroquinolone-resistant Escherichia coli (Grade 1-A). Do not use moxifloxacin for the 
empiric treatment of children unless no other options are available (Grade 1-C).

- Use ciprofloxacin plus metronidazole for empiric therapy of lower-risk adults with CA-IAI, but use with caution in areas where there is a high incidence of fluoroquinolone-resistant E. coli (Grade 1-A). Consider use of the regimen for empiric therapy of lower-risk children if other options are not suitable (Grade 2-B).

- Consider use of levofloxacin plus metronidazole as an option for empiric therapy of lower-risk adults, if use of a fluoroquinolone is warranted and it is the only fluoroquinolone available (Grade 2-C). Consider use of this regimen for empiric therapy of lower-risk children if other options are not suitable (Grade 2-C).

\section{H. Tigecycline}

- Do not use tigecycline for empiric therapy under most circumstances (Grade 1-B). Consider use of this agent for therapy of adult patients with resistant pathogens, particularly as a component of a combination regimen, if other agents are not suitable (Grade 2-B).

\section{Anti-anaerobic agents}

- Use metronidazole as the preferred anti-anaerobic agent in combination regimens for empiric therapy in adults and children (Grade 1-B).

- Do not use clindamycin as an anti-anaerobic agent in combination regimens for the empiric treatment in adults and children unless metronidazole cannot be used (Grade 2-B). Consider use of clindamycin in children under one month of age (Grade 2-C).

\section{J. Anti-enterococcal and anti-staphylococcal agents}

- Consider use of ampicillin for empiric or pathogendirected therapy of susceptible enterococcal strains in higher-risk adults and children (Grade 2-B).

- Consider use of vancomycin for empiric or pathogendirected therapy of vancomycin-susceptible Enterococcus faecium or methicillin-resistant Staphylococcus aureus (MRSA) in higher risk adults and children (Grade 2-B). Include vancomycin in aztreonam-based regimen for coverage of gram-positive organisms (Grade 2-B).

- Consider use of linezolid or daptomycin for empiric or pathogen-directed therapy of infections from vancomycin-resistant Enterococcus spp. (VRE) and as an alternative to vancomycin for infections from MRSA in adults and children (Grade 2-B).

\section{K. Antifungal agents}

- Do not use amphotericin B or its lipid formulations routinely for empiric or pathogen-directed management of intra-abdominal candidiasis in adults or children (Grade 2-B).

- Consider use of fluconazole for preemptive and for pathogen-directed therapy of susceptible strains of Candida albicans in non-critically ill adults and children (Grade 2-B).

- Consider use of voriconazole for empiric or pathogendirected therapy of fluconazole-non-susceptible strains of Candida in non-critically ill adults and in children older than one month of age (Grade 2-B).

- Use an echinocandin (anidulafungin, caspofungin, or micafungin) for empiric or pathogen-directed treatment of infections from Candida spp. in severely ill adults and children (Grade 1-B).

\section{Oral antimicrobial agents}

Substitution of oral for IV antibiotic agents in patients with IAI may be considered under selected circumstances. Specific recommendations are:

- Use selected oral agents with good bioavailability as a substitute for IV agents for therapy of patients with return of adequate gastrointestinal function. Use oral antibiotics only to complete a short course of treatment and not to prolong antimicrobial use beyond current recommendations (Grade 1-B).

- Consider use of oral amoxicillin-clavulanic acid as an option to complete a short course of antimicrobial therapy in adults and children (Grade 2-B).

- Consider use of oral moxifloxacin as an option to complete a short course of antimicrobial therapy in adults (Grade 2-B). Do not use oral moxifloxacin in children unless no other option is available (Grade 1-B).

- Use oral ciprofloxacin plus metronidazole to complete a short course of antimicrobial therapy in adults (Grade 1-B). Consider use of oral ciprofloxacin plus metronidazole to complete a short course of antimicrobial therapy in children if other options are not suitable (Grade 2-B).

- Consider use of oral levofloxacin plus metronidazole, an oral first-, second-, or third-generation cephalosporin plus metronidazole, or oral trimethoprim-sulfamethoxazole plus metronidazole as potential options to complete a short course of antibiotic therapy in adults and children if other oral agents are not suitable (Grade 2-C).

\section{Selection of empiric antimicrobial therapy for adult patients with CA-IAI}

The selection of specific empiric IV antimicrobial therapy for the treatment of patients with CA-IAI should be based on principles of antimicrobial stewardship, using broaderspectrum agents primarily for seriously ill patients. Specific recommendations are:

\section{A. Lower-risk patients with CA-IAI}

- Treat lower-risk patients with narrower-spectrum antimicrobial agents having activity against the usual gram-negative Enterobacteriaceae, aerobic streptococci, and obligate anaerobic micro-organisms associated with these infections (Grade 1-A). Do not routinely use broader-spectrum or additional agents to provide antipseudomonal, anti-enterococcal coverage (Grade 1-A), or antifungal therapy (Grade 2-B).

- Use cefotaxime or ceftriaxone plus metronidazole or ertapenem as the preferred agents for initial empiric therapy of lower-risk patients (Grade 1-A). Consider use of cefuroxime plus metronidazole or cefoperazonesulbactam, where available, as alternatives (Grade 2- 
B). Use ciprofloxacin plus metronidazole or moxifloxacin monotherapy for patients who have serious $\beta$ lactam allergies (Grade 1-A). Consider use of levofloxacin plus metronidazole as an alternative if no other fluoroquinolone is available (Grade 2-C).

- Use agents recommended for lower-risk patients with perforated appendicitis, unless they meet criteria as higher-risk patients or at risk for having resistant pathogens (Grade 1-A).

\section{B. Higher-risk patients with CA-IAI}

- Treat higher-risk patients with broader-spectrum empiric antimicrobial agents to ensure coverage of less common gram-negative pathogens potentially involved in these infections (Grade 2-C).

- Use piperacillin-tazobactam, doripenem, imipenemcilastatin, meropenem, or cefepime plus metronidazole as the preferred agents for initial empiric therapy of higher-risk patients (Grade 2-A). Consider use of ceftazidime plus metronidazole as an alternative regimen for these patients (Grade 2-B). Consider use of aztreonam plus metronidazole plus vancomycin as an option for higher-risk patients with a severe reaction to $\beta$-lactam agents (Grade 2-B). Do not add an adjunctive aminoglycoside or fluoroquinolone to a $\beta$-lactam agent for empiric treatment of higher-risk patients (Grade 1-B).

- Consider use of added ampicillin or vancomycin for empiric anti-enterococcal treatment in higher-risk patients if the patient is not being treated with piperacillintazobactam or imipenem-cilastatin (Grade 2-B).

- Do not use antifungal agents routinely for empiric therapy of higher-risk patients (Grade 1-B). Consider use of antifungal agents for empiric therapy of critically ill patients with an upper gastrointestinal source (Grade 2-B).

C. Other considerations for empiric antimicrobial therapy of patients with CA-IAI

- Consider use of fluoroquinolone-based regimens for initial empiric therapy of lower-risk patients who have major reactions to $\beta$-lactam antibiotics (Grade 2-B). Consider use of an aztreonam-based regimen for initial empiric therapy of higher-risk patients who have major reactions to $\beta$-lactam antibiotics (Grade 2-B). Consider use of a non-penicillin $\beta$-lactam for empiric therapy of patients for whom a severe penicillin allergy has not been documented and for whom the risk-benefit ratio is believed acceptable (Grade 2-B).

- Do not use cephalosporin-, aztreonam-, or fluoroquinolonebased regimens for empiric therapy of patients who reside in geographic areas where there is a high prevalence of extended-spectrum $\beta$-lactamase (ESBL)producing Enterobacteriaceae in the community (Grade 1-B). Use ertapenem for empiric therapy in lower-risk patients or a broad-spectrum carbapenem for higherrisk patients who reside in such areas (Grade 1-B).

- Consider use of locally available IV or oral agents having activity against common intra-abdominal pathogens for empiric therapy in patients who reside in geographic areas where there are major resource limitations (Grade 2-C).

\section{Selection of empiric antimicrobial therapy for adult patients with HA-IAI}

Because patients with HA-IAI are at risk for infection from resistant organisms, additional antimicrobial agents may lessen the risk of inadequate initial therapy and subsequent treatment failure. The recommendations include:

\section{A. General approach}

- Assess patients with respect to their separate risks of infection from Enterococcus spp., MRSA, resistant gram-negative bacilli, and Candida spp. (Grade 2-B).

- Use the broader-spectrum agents recommended for higher-risk patients with CA-IAI for initial empiric therapy of patients with HA-IAI. Consider addition of other empiric agents based on the patient's risk for an infection from Enterococcus spp., MRSA, resistant gram-negative bacilli, and Candida spp. (Grade 2-B).

\section{B. Anti-enterococcal therapy}

- Identify patients with HA-IAI who have post-operative infections, recent exposure to broad-spectrum antimicrobial therapy, signs of severe sepsis or septic shock, or known to be colonized with VRE as at risk for infection with Enterococcus spp. (Grade 2-B).

- Consider use of vancomycin or teicoplanin for empiric therapy of HA-IAI in patients at risk for infection from Enterococcus spp. Consider use of linezolid or daptomycin for empiric therapy of patients known to be colonized with or at high risk for infection with VRE (Grade 2-B).

\section{Anti-staphylococcal therapy}

- Identify patients with HA-IAI with multiple healthcareassociated risk factors for MRSA colonization, including advanced age, co-morbid medical conditions, previous hospitalization or surgery, and significant recent exposure to antibiotic agents, or known to be colonized with MRSA at risk for infection due to MRSA (Grade 2-B).

- Consider use of vancomycin or teicoplanin, where available, or linezolid or daptomycin as alternatives, for empiric therapy of patients known to be colonized or at high risk for infection with MRSA (Grade 2-B).

\section{Antibacterial therapy for resistant gram-negative organisms}

- Identify patients who have received substantial previous broad-spectrum antimicrobial therapy, had prolonged hospitalizations, undergone multiple invasive interventions, or known to have been colonized or infected with a resistant gram-negative organism at risk for infection from a resistant gram-negative pathogen (Grade 2-B). Consult local epidemiologic data and antibiograms for assistance in selecting empiric antimicrobial therapy in patients considered at risk for infection with resistant gram-negative pathogens (Grade 2-B).

- Consider use of a broad-spectrum carbapenem, or ceftolozane-tazobactam or ceftazidime-avibactam as alternatives, for empiric therapy of patients at risk for infection with ESBL-producing Enterobacteriaceae (Grade 2-B). 
- Consider use of a broad-spectrum carbapenem, with ceftazidime-avibactam as an alternative, for empiric therapy of patients at risk for infection with Amp C- $\beta$ lactamase-producing Enterobacteriaceae (Grade 2-B).

- Consider use of combinations of a carbapenem or ceftazidime-avibactam as an alternative, an aminoglycoside, a polymyxin, and/or tigecycline for empiric therapy of patients at risk for infection with carbapenem-resistant Enterobacteriaceae (Grade 2-B).

- Consider use of combinations of a $\beta$-lactam antibiotic, including ceftolozane-tazobactam, an aminoglycoside, and/or a polymyxin, for empiric therapy of patients at risk for infection with multi-drug resistant (MDR)-, extensively drug resistant (XDR)-, or pandrug resistant (PDR)-strains of $P$. aeruginosa (Grade 2-B). Consider use of combinations of a carbapenem, an aminoglycoside, a polymyxin, and/or tigecycline for empiric therapy of patients at risk for infection with MDR-, XDR-, or PDR-strains of Acinetobacter spp. (Grade 2-B).

\section{E. Antifungal therapy}

- Identify patients with HA-IAI because of upper gastrointestinal perforations, recurrent bowel perforations, surgically treated pancreatitis, those who have received prolonged courses of broad-spectrum antibiotic therapy, and those who are known to be heavily colonized with Candida at increased risk for infection from Candida spp. (Grade 2-B). Consider patients found to have yeast on a Gram stain of infected peritoneal fluid or tissue as having an infection with Candida spp. (Grade 2-B).

- Use an echinocandin (anidulafungin, caspofungin, or micafungin) for empiric therapy of severely ill patients at risk for infection with Candida spp. (Grade 1-B). Consider use of fluconazole for antifungal therapy of less severely ill patients at risk for infection with Candida spp. (Grade 2-B). Consider use of an echinocandin or voriconazole for empiric therapy of patients at risk for infection with a fluconazole-resistant strain of Candida (Grade 2-B).

\section{Timing of antimicrobial therapy}

Antimicrobial therapy for patients with IAI needs to be timed to optimize management of the infection as well as prevent secondary infections following source control.

- Initiate empiric antimicrobial therapy within one hour, if possible, once a diagnosis of IAI is made in patients presenting with sepsis or septic shock (Grade 2-B). Initiate antimicrobial therapy as soon as feasible in other patients with IAI, taking into account plans for subsequent source control (Grade 2-C).

- Re-administer an antimicrobial agent within one hour before the start of a source control procedure if two half-lives of the agent have passed at the time the intervention is initiated (Grade 1-B).

\section{Dosing of antimicrobial agents in adult patients}

Antimicrobial dosing in adult patients with IAI should be optimized based on the patient's physiologic conditions and co-morbidities. Specific recommendations related to antimicrobial dosing and administration include:
- Use standard dosages of antimicrobial agents for lowerrisk patients who are not severely obese and who do not have substantial renal or hepatic impairment (Grade 1B). Consider use of higher dosages of antimicrobial agents in selected higher-risk patients (Grade 2-B).

- Use adjusted dosages of antimicrobial agents, based on available clinical outcome data, standard pharmacokinetic parameters, and therapeutic drug monitoring, where applicable, in patients with significant renal impairment (Grade 2-B).

- Use adjusted dosages of selected, hepatically eliminated or metabolized antimicrobial agents in patients with substantial hepatic impairment (Grade 2-B).

- Consider use of adjusted dosages of selected antimicrobial agents, based on available clinical outcome data and pharmacokinetic parameters, in obese patients (Grade 2-B).

- Do not use prolonged or continuous infusion of $\beta$ lactam antibiotic agents routinely in all patients, but consider use of these approaches as options in critically ill patients and those at risk for infection with resistant gram-negative pathogens (Grade 2-B).

\section{Duration of antimicrobial therapy}

The duration of antimicrobial therapy in the patient with IAI needs to be specific for each clinical condition. Specific recommendations include:

- Do not use antibiotic agents to prevent infection in patients with severe or necrotizing pancreatitis (Grade $1-\mathrm{B})$.

- Consider deferral of antibiotic therapy in lower-risk patients with uncomplicated acute colonic diverticulitis (Grade 2-B).

- Limit antimicrobial therapy to no more than 24 hours in patients with traumatic bowel perforations operated on within 12 hours (Grade 1-A), patients with gastroduodenal perforations operated on within 24 hours (Grade 1-C), patients with acute or gangrenous appendicitis in the absence of perforation (Grade 1-A), patients with acute or gangrenous cholecystitis in the absence of perforation (Grade 1-A), and patients with ischemic, non-perforated bowel (Grade 1-C).

- Limit antimicrobial therapy to four days $(96 \mathrm{~h})$ in patients who have had adequate source control (Grade $1-\mathrm{A})$.

- Consider limiting antimicrobial to 5-7 days in patients with established IAI in whom a definitive source control procedure is not performed. Consider use of clinical parameters of fever, leukocytosis, and adequacy of gastrointestinal function to determine whether antimicrobial therapy can be discontinued sooner. Re-assess patients who do not respond fully to antimicrobial therapy within 5-7 days for a potential source control intervention (Grade 2-C).

- Consider limiting antimicrobial therapy to seven days in patients with secondary bacteremia because of IAI, who have undergone adequate source control and are no longer bacteremic (Grade 2-B).

- There are insufficient data to evaluate duration of therapy in patients receiving immunosuppressive medications (no recommendation). 


\section{Pathogen-directed antimicrobial therapy}

Changing antimicrobial therapy based on culture results should be considered in selected patients. Specific recommendations include:

- Do not change antimicrobial therapy on the basis of culture results in lower-risk patients who have had a satisfactory clinical response to source control and empiric therapy (Grade 1-B).

- Consider modification of antimicrobial therapy in higher-risk patients if culture results identify organisms resistant to the initial empiric regimen and further antimicrobial therapy is planned (Grade 2-C).

- There are insufficient data to make a recommendation regarding modification of antimicrobial therapy in patients who have a highly resistant organism isolated as a minor component of a mixed peritoneal culture (no recommendation).

- Routinely de-escalate or streamline antimicrobial therapy in higher-risk patients to the narrowest-spectrum agent or agents having activity against the isolated micro-organisms once definitive culture results are available (Grade 1-B).

\section{Treatment failure}

Treatment of patients who fail initial therapy follows the same principles used in initial management of IAI. Specific recommendations include:

\section{A. Source control for treatment failure}

- Use measures of ongoing or progressive systemic inflammation or organ system dysfunction to identify patients with likely source control failure (Grade 1-B).

- Assess patients for source control failure if there is progressive organ dysfunction within the first 2448 hours after source control, if there is no clinical improvement in organ dysfunction 48 hours or more after source control, or if there are persistent signs of inflammation 5-7 days after source control (Grade 2-C).

- Consider abdominal exploration in patients who deteriorate clinically or fail to improve within 48-72 hours of the initial procedure (Grade 2-C). Use computed tomography scanning, with percutaneous aspiration or drainage of any potentially infected fluid collections, in patients suspected of treatment failure after 48-72 hours of the initial source control procedure (Grade 1-B).

- Use the least invasive approach that will achieve definitive source control or sufficiently control the infection to allow resolution of the inflammatory response and organ dysfunction (Grade 1-B).

- Undertake further source control within 24 hours when source control failure is identified (Grade 2-C), but as soon as feasible in patients with physiologic instability or progressive organ dysfunction (Grade 2-B).

- Obtain routine peritoneal cultures in patients with source control failure so that pathogen-directed antimicrobial therapy can be utilized (Grade 1-C).

\section{B. Antimicrobial therapy for treatment failure}

- Do not routinely change antimicrobial therapy when patients have early treatment failure and undergo repeat source control within 48 hours of the initial source control intervention (Grade 2-C). Consider altering antimicrobial therapy, using an alternative antibiotic class if feasible, in patients who have late treatment failure (Grade 2-C).

- Consider discontinuation of antimicrobial therapy in patients with clinical evidence of treatment failure but negative results of imaging studies for recurrent or persistent IAI (Grade 2-B).

- Consider a trial of further antimicrobial therapy in patients with clinical evidence of treatment failure and imaging studies showing ongoing intra-abdominal inflammation; if there is no clinical response to this antimicrobial trial within a few days, discontinue antimicrobial therapy and re-instate only if there is evidence of clinical deterioration (Grade 2-C).

- Consider continuation of antimicrobial therapy in patients with clinical evidence of treatment failure and imaging studies showing recurrent or persistent IAI, in whom further source control cannot be achieved; discontinue antimicrobial therapy when clinical signs of systemic inflammation or organ dysfunction abate (Grade 2-C). Monitor these patients for resistant pathogens, adjusting antimicrobial therapy as necessary (Grade 2-C).

\section{Treatment of pediatric IAI}

Management of IAI in pediatric patients with IAI follows the same general principles as treatment of adult patients. Specific recommendations on treatment of pediatric patients with IAI include:

- Use cefotaxime or ceftriaxone plus metronidazole or ertapenem as preferred agents for empiric therapy of lower-risk pediatric patients greater than one month of age (45 wks post-conceptional age, Grade 1-A). Consider use of cefuroxime plus metronidazole or cefoperazonesulbactam, where available, as alternatives (Grade 2-B). Consider use of ciprofloxacin plus metronidazole, or levofloxacin plus metronidazole if no other fluoroquinolone is available, for empiric treatment of selected pediatric patients if other agents cannot be used, particularly for those patients with life-threatening $\beta$-lactam reactions (Grade 2-B).

- Use piperacillin-tazobactam, imipenem-cilastatin, or meropenem as the preferred agents for empiric therapy of higher-risk pediatric patients greater than one month of age (45 wks post-conceptional age) with CA-IAI (Grade 2-A) or with HA-IAI (Grade 2-B). Consider use of ceftazidime or cefepime plus metronidazole as alternatives (Grade 2-B). Consider use of aztreonam plus metronidazole plus vancomycin as an option if other agents cannot be used, particularly for those with lifethreatening $\beta$-lactam reactions (Grade 2-B). Consider addition of ampicillin or vancomycin as empiric antienterococcal therapy of higher-risk patients if the patient is not being treated with piperacillin-tazobactam or imipenem-cilastatin (Grade 2-B).

- Limit antimicrobial therapy to five days $(120 \mathrm{~h})$ in pediatric patients older than one month (45 wks postconceptional age) who have had adequate source control (Grade 1-A). 
- Use oral antimicrobial agents with good bioavailability to complete a five-day course of therapy in patients with adequate source control when feasible, but do not use oral antimicrobial agents to extend total antimicrobial duration beyond five days (Grade 1-B).

- Do not use additional outpatient IV antimicrobial therapy in patients with adequate source control unless administered to complete a total antimicrobial course of five days (Grade 1-B).

- Treat pediatric patients with failure of treatment in an analogous fashion to adult patients with treatment failure (Grade 1-C). Use the least invasive means of providing adequate source control and a standard course of IV antibiotic agents, preferably with a change in the antibiotic class, to treat these patients (Grade 2C). Do not extend a course of IV antibiotic agents beyond seven days in children with perforated appendicitis who have a post-operative abscess (Grade 1-C).

- Employ either laparotomy or peritoneal drainage as source control in addition to antimicrobial therapy for pediatric patients less than one month of age $(45 \mathrm{wks}$ post-conceptional age) with necrotizing enterocolitis or intestinal perforation (Grade 1-A).

- Use ampicillin, gentamicin, and either metronidazole or clindamycin; ampicillin, cefotaxime, and either metronidazole or clindamycin; or meropenem in pediatric patients less than one month of age ( $45 \mathrm{wks}$ post-conceptional age). Consider use of vancomycin instead of ampicillin if there is suspected infection with penicillin-resistant Enterococcus spp. or MRSA. Consider use of fluconazole or amphotericin $\mathrm{B}$ if there is a suspected infection with Candida spp. (Grade 2-C).

- Use a 7-10 day course of antimicrobial therapy in pediatric patients less than one month of age (45 wks post-conceptional age), particularly for those with necrotizing enterocolitis (Grade 2-C).

- Use standard pediatric dosages for various antimicrobial agents for lower-risk pediatric patients with CAIAI (Grade 1-B). Consider use of higher pediatric dosages, where applicable, for higher-risk patients with CA-IAI and those with HA-IAI (Grade 2-C).
$\mathbf{I}$ NTRA-ABDOMINAL INFECTION (IAI) is a common disease process managed primarily by surgical practitioners. It is associated with substantial morbidity and death, despite therapeutic advances made over the past decades. Key components of the management of these infections include expeditious diagnosis, early resuscitation of the patient, timely and appropriate source control, and adequate antimicrobial therapy directed against the micro-organisms involved in the infection.

The Surgical Infection Society (SIS) has released various evidence-based guidelines for the management of these infections. The initial guideline developed by the Antimicrobial Agents Committee (now the Therapeutics and Guidelines Committee) was published in 1992 [1]. A subsequent revision based on published evidence through early 2001 was published in 2002 [2]. Accompanying this publication was a compilation of the evidence used to develop the recommendations [3]. These initial guidelines focused primarily on appropriate antimicrobial therapy for these infections. Subsequently, in 2010, a more comprehensive document written jointly by the SIS and the Infectious Diseases Society of America (IDSA) was published, which included some recommendations regarding diagnosis, initial resuscitation, and source control for these infections, as well as treatment of children with IAI [4]. This guideline revised both the 2002 SIS guideline and a similar guideline published by the IDSA in 2003 [5].

Since the 2010 guideline, additional challenges have arisen in the management of these infections. With the advancing age and increased burden of chronic disease in the population, IAI is being diagnosed more commonly and managed in patients with impaired host defenses and limited physiologic reserves. In addition, the prevalence of resistant bacteria and fungi has been growing worldwide, with resistant microorganisms identified not only in patients whose infections arise in the healthcare setting, but also in patients in whom their infections develop in the community. This has led to declining efficacy of some antimicrobial agents traditionally used to treat these patients.

Nonetheless, advances have been made in the management of IAI as well. An increasingly standardized and successful approach to the resuscitation of patients with sepsis, including those with IAI, has been provided through the various iterations of the Surviving Sepsis Campaign guidelines [6]. More targeted and less morbid techniques for achieving source control are being increasingly used in patients with IAI. Finally, a few newer antimicrobial agents are now available that have activity against some resistant pathogens, although the problem of resistance remains a major problem for managing nearly any type of infection.

This revision of the 2010 guideline was undertaken by a task force from the SIS including members of the Therapeutics and Guidelines Committee as well as additional individuals with expertise in the subject. Because the previous guideline did not include an in-depth evidence review, the task force elected to review not only the literature related to management of IAI published since dissemination of the 2010 guideline, but to also re-analyze the literature published between 2001 and 2008 that had been used in part for the previous revision. The guideline was also constructed in a manner such that it might be amenable for use in an algorithmic format for treating patients with IAI.

Previous guidelines have used various approaches to grading the quality of evidence and the strength of the recommendations. Increasingly, the GRADE (Grades of Recommendation Assessment, Development, and Evaluation) nomenclature has been used for guideline development. The GRADE approach calls for a clearly transparent methodology to guideline development. It also separates issues related to the quality of the evidence from the overall strength and importance of the recommendation. Evidence is characterized according to the quality of the study design and its execution, but also considers problems of consistency, directness, and potential bias in grading quality [7]. The recommendations themselves are strongly influenced by the quality of the evidence behind them, but also take into consideration the balance between desirable and undesirable effects, values and preferences in different settings, and the resources needed to fully implement the recommendation [8]. 
Table 1. Class of Evidence

\begin{tabular}{|c|c|c|}
\hline $\mathrm{A}$ & $\begin{array}{l}\text { High quality } \\
\text { evidence }\end{array}$ & $\begin{array}{l}\text { The evidence was primarily obtained from RCTs, meta-analyses of such trials, } \\
\text { or methodologically sound epidemiologic studies. If the preponderance of evidence } \\
\text { is based on studies that do not directly address the question being posed, the overall } \\
\text { grade is downgraded to B or C. If there are conflicts in Class A data, the evidence grade } \\
\text { is lowered to B or C, depending on the degree of conflict. }\end{array}$ \\
\hline B & $\begin{array}{l}\text { Moderate } \\
\text { quality } \\
\text { evidence }\end{array}$ & $\begin{array}{l}\text { The evidence was obtained from lower quality prospective studies, retrospective case } \\
\text { control studies, and large observational, cohort, or prevalence studies, and was based } \\
\text { on clearly reliable data. If there are significant conflicts in Class B data, the evidence } \\
\text { grade is lowered to C. }\end{array}$ \\
\hline $\mathrm{C}$ & $\begin{array}{l}\text { Weak quality } \\
\text { evidence }\end{array}$ & $\begin{array}{l}\text { The evidence was obtained from smaller observational studies, studies relying on retrospective } \\
\text { or less reliable data, authoritative opinions expressed in reviews, or expert opinions } \\
\text { of task force members. }\end{array}$ \\
\hline None & $\begin{array}{r}\text { Insufficient } \\
\text { evidence }\end{array}$ & $\begin{array}{l}\text { There was little or no relevant evidence to address a question, or the evidence reviewed } \\
\text { was highly conflicting. }\end{array}$ \\
\hline
\end{tabular}

$\mathrm{RCT}=$ randomized controlled trial.

The current guideline uses this basic approach to grading the evidence and the resultant recommendations, as summarized in Tables 1 and 2. Because of the limited quantity of methodologically rigorous studies investigating key questions in the management of IAI, the task force did not undertake a detailed statistical analysis for most of these recommendations, but relied on a process of iterative consensus among task force members to develop the recommendations and their final grading.

\section{Methods}

The task force undertook a formalized process of guideline development and review, which is outlined here. This process was based on that outlined by the GRADE Working Group [9].

The initial selection of the task force chair and working group leaders was made by the Executive Council of the SIS. These individuals then named additional SIS members identified as content experts in the management of IAI to the task force, supplemented by the members from the Therapeutics and Guidelines Committee with an interest in the topic. An initial series of teleconferences and one face-toface meeting were held in 2014 and 2015, but much of the initial and subsequent work was done via electronic mail.

Members of the task force first agreed on the scope of the guideline and developed an outline of a series of topics for review (Table 3). Working groups then developed specific questions to be addressed under each of these topics, which were supplemented by additional suggestions from members of the task force. These questions were then refined by iterative review until a consensus was achieved on the specific questions that would be investigated.

Evidence was then collected to investigate these questions. The primary tactic employed was a systematic search of the Medline database using various strategies in an effort to identify all literature related to IAI published between 2007 and mid-2014. Abstracts of more than 8,000 articles were subjected to a first review by task force members to identify articles relevant to the current revision. These publications were combined with literature published between 2000 and 2007, which had been selected from a similar search of the Medline database performed for preparation of the previous guideline.

Secondary searches were performed to supplement the primary search when evidence was found to be lacking for examination of a specific question. The focus of such searches was prevention or management of infections other than IAI, and identification of side effects or complications related to specific antimicrobial agents or therapeutic approaches. In addition, literature relevant to the topic was identified during systematic evaluations of review articles for pertinent references, including those published as part of the Cochrane Database of Systematic Reviews [10].

\section{Table 2. Rating Scale for Recommendations}

\begin{tabular}{|c|c|c|}
\hline 1 & $\begin{array}{l}\text { Strong } \\
\quad \text { recommendation }\end{array}$ & $\begin{array}{l}\text { The task force concluded that the intervention is a desirable approach for the care of those } \\
\text { patients to whom the question applies. This rating is generally based on moderate to } \\
\text { high quality evidence. The conclusion is unlikely to be changed with future research. } \\
\text { The magnitude of the effect is also sufficient to justify the recommendation. A strong } \\
\text { recommendation was also used to describe interventions that are likely to have a } \\
\text { significant effect on patient outcome, even if based on weak evidence. These } \\
\text { recommendations are prefaced as "We recommend ...". }\end{array}$ \\
\hline 2 & $\begin{array}{l}\text { Weak } \\
\text { recommendation }\end{array}$ & $\begin{array}{l}\text { The task force concluded that the intervention is a reasonable approach for the care } \\
\text { of patients. Not all patients and clinicians, however, would necessarily want to follow } \\
\text { the recommendation. A decision not to follow the recommendation is unlikely to result } \\
\text { in a major adverse outcome. This rating was generally based on weak to moderate quality } \\
\text { evidence. Both the magnitude of the treatment effect and its direction might be altered } \\
\text { by future research. These recommendations are prefaced as "We suggest...". }\end{array}$ \\
\hline None & $\begin{array}{l}\text { No } \\
\text { recommendation }\end{array}$ & $\begin{array}{l}\text { The evidence was considered inadequate or too inconsistent to allow any meaningful } \\
\text { conclusion to be reached. }\end{array}$ \\
\hline
\end{tabular}


Table 3. Guideline Topics

Risk assessment
Source control
Microbiologic evaluation
Intravenous antimicrobial agents
Oral antimicrobial agents
Selection of empiric antimicrobial therapy for adult patients
with CA-IAI
Selection of empiric antimicrobial therapy for adult patients
with HA-IAI
Timing of antimicrobial therapy
Dosing of antimicrobial agents in adult patients
Duration of antimicrobial therapy
Pathogen-directed antimicrobial therapy
Treatment failure
Management of pediatric IAI

CA-IAI = community-acquired intra-abdominal infection; HAIAI = healthcare- or hospital-acquired intra-abdominal infection; $\mathrm{IAI}=$ intra-abdominal infection.

Before final review of the guideline, an additional comprehensive Medline search was performed to uncover relevant articles published between 2014 and early 2016 that had not been already identified during the previous or supplementary searches. Selected publications from before 2000 were added to the database when necessary. The final literature database included 778 articles identified during the primary screening process, 565 identified via secondary and supplementary searches, and 89 identified in the final search of the most recent literature.

The literature database was screened for randomized controlled trials (RCTs) related to use of antimicrobial therapies for treatment of patients with IAI published between 2000 and 2016. These trials underwent formal review; studies that enrolled patients with multiple types of infections were reviewed if outcomes in patients with IAI were reported separately from the outcomes of all patients with infections. The RCTs related to surgical or other interventional approaches were selected for formal review only if they related to strategies for treating patients with IAI. The task force elected not to study questions related to technical aspects of managing specific disease entities, such as studies comparing laparoscopic versus open approaches for acute appendicitis.

The RCTs were reviewed by at least two members of the task force, using the criteria employed during the previous evidence review [3]. The primary end point assessed was clinical cure in the clinically evaluable population at the time point (generally a test of cure visit) specified by the specific trial design; where available, clinical cure in the modified intent-to-treat population (those receiving at least one dose of study drug) was also used. Severity of illness in the study populations was gauged by overall death, the percentage of patients having an appendiceal source of infection, and Acute Physiology and Chronic Health Evaluation II (APACHE II) scores, where reported. Quality was graded on a scale of 0-5 based on adequacy of randomization, blinding, and description of patients excluded from the study, according to the system of Jadad et al. [11]. Quality was also assessed based on whether or not patients enrolled in the trial met the criteria for complicated IAI, as specified by IDSA criteria [12]. Discrepancies between the two reviewers were resolved by a third reviewer.
Working groups were obligated to use the data from all relevant RCTs when formulating recommendations for specific questions. These data were evaluated according to GRADE criteria for precision, directness, consistency, and risk of bias [7], but formal GRADE tables were not prepared. This assessment was incorporated into the final evidence grade applied to each recommendation (Table 2).

Data from RCTs were available for only a small fraction of questions developed by the subgroups. Thus, heavy reliance was placed on data from other types of studies to formulate many recommendations. Working groups used selected publications from the literature database for these questions, with the choice of specific references being based on the perceived quality and relevance of the study to the question at hand. Because no generally accepted system exists for evaluating quality of these studies, they were not objectively graded. Summaries of RCTs and selected additional literature pertinent to treatment of IAI are provided in the Supplementary Tables, which are available on the Surgical Infections website (www.liebertpub.com/overview/surgical-infections/53/).

Based on these literature reviews, individual working groups drafted and graded provisional recommendations. The GRADE criteria of quality of evidence, balance between desirable and undesirable effects, values and preferences, and resource allocation [8] were used for this, emphasizing the first two criteria (Table 3). The entire task force repeatedly reviewed and refined these recommendations until a final consensus was achieved.

The guideline was then submitted to independent reviewers from the SIS who were not members of the task force. The task force responded to these critiques by either modifying the specific recommendation or leaving it unchanged, providing a detailed rationale for its decision. After a final vote was taken on all recommendations, the guideline along with supporting materials was presented to the Executive Council of the SIS for approval as an official guideline of the SIS. Documentation regarding individual votes or recusals on recommendations by task force members as well as minutes of the SIS Executive Council deliberations are maintained by the Executive Director of the SIS.

\section{Background}

\section{Scope of the guideline}

Intra-abdominal infection refers to a wide variety of infections encountered in clinical practice. The term, however, is used generally in the context of disease processes within the abdominal cavity treated with some type of mechanical intervention, such as a surgical procedure. It has been traditional to separate IAI into uncomplicated and complicated IAI. With some pathologic entities, however, the distinction between uncomplicated and complicated IAI becomes somewhat arbitrary, as will be more fully discussed in the subsequent section.

Past IAI guidelines have focused primarily on complicated IAI. Although the vast majority of this document emphasizes treatment of patients with complicated IAI, it also includes some recommendations relevant to the treatment of patients with uncomplicated IAI and some non-infectious disease processes that can lead to IAI. When referring to uncomplicated IAI, the guideline will usually describe the specific disease entity, such as acute appendicitis, acute cholecystitis, 
or uncomplicated diverticulitis, rather than combining these disparate infections under the term "uncomplicated IAI." Throughout the document, the term "IAI" should generally be considered synonymous with the term "complicated IAI."

As with previous guidelines, management of certain disease entities occurring in the abdomen is not considered in this guideline, including primary peritonitis, catheter-related peritonitis, and infections in solid abdominal organs arising from hematogenous spread. Diseases developing as a result of transmural enteritis or inflammatory bowel disease, and diseases primarily involving the genitourinary tract have also been excluded.

Management of IAI generally involves expeditious diagnosis, initial resuscitation of the patient, source control, and antimicrobial therapy. Diagnosis of IAI is usually undertaken by other persons, such as emergency medicine physicians or primary care providers, and may use various approaches, depending on the actual disease entity being investigated. The task force elected not to review this subject. Initial resuscitation of the patient is frequently the responsibility of a number of persons, including surgeons as well as emergency medicine providers, anesthesiologists, and critical care physicians. Guidance on the care of the patient with sepsis and septic shock, including patients with IAI, is provided in the Surviving Sepsis Campaign guideline [6], which is currently being updated. The reader is referred to that guideline, which has been endorsed by the SIS, for further information on this topic.

As emphasized in the Surviving Sepsis Campaign guideline, early source control and provision of appropriate empiric antimicrobial therapy are key to the survival and recovery of seriously ill patients with infection, including those with IAI. The task force has elected to focus on these issues in this guideline, because these are the aspects of management most directly under the control of the surgical practitioner. Source control refers to a large variety of mechanical techniques for treating patients with IAI, with a goal of decreasing the bacterial inoculum and allowing the patient's host defenses to control the infection. This guideline expands the discussion of source control somewhat compared to the previous guideline. Nonetheless, because of the wide variety of potential approaches to source control, only general concepts of source control are addressed, and interventional therapies for specific disease entities have been left to be addressed by more focused guidelines. Antimicrobial therapy is likewise approached from a relatively broad perspective. With the advent of increasingly resistant microbial strains in both the hospital and community environments, however, the treatment of patients with resistant or opportunistic micro-organisms has been given further weight. Finally, although initial treatment using an evidence-based approach is frequently successful, it fails in approximately $20 \%$ of patients. Patients with initial treatment failure are at increased risk for further adverse outcomes. Thus, treatment of patients with failure of treatment has been given additional emphasis in this guideline.

The goal of this document is to provide guidance to clinicians by describing reasonable approaches to the use of therapeutic modalities. An important consideration stressed in this guideline is that most management decisions should be tempered according to an individualized assessment of the patient, taking into account not only the risk of the specific interventions being contemplated, but also the likelihood of a poor outcome if inadequate therapeutic efforts are employed. It is also important to recognize, however, that collective decisions on the use of these modalities have impacts on the healthcare system and the overall population as well. For instance, widespread use of expensive imaging or therapeutic equipment, particularly in areas of substantial resource limitations, may limit the opportunity to use other therapies of equal or greater value to the community. Hence, the task force has attempted to take into account the cost-effectiveness of certain interventions in its recommendations. Further, with regard to antimicrobial therapy, overuse of antimicrobial resources leads to the development of microbial resistance and loss of effective anti-infective agents for all. The principles of antimicrobial stewardship have therefore been stressed throughout this document.

\section{Definitions}

Much of the published literature regarding management of IAI uses the concept of uncomplicated and complicated IAI. Traditionally, infections limited to a hollow viscus were called uncomplicated IAI, whereas those that extended into a normally sterile area of the abdomen, such as the peritoneal cavity, mesentery, retroperitoneum, another abdominal organ, or the abdominal wall, were defined as complicated IAI $[4,13]$. Nonetheless, there are borderline conditions, such as localized colonic diverticulitis, which may be difficult to categorize as a complicated or uncomplicated IAI. Complicated IAI have also traditionally been described as those disorders managed with a source control procedure; in part, this has been to satisfy the requirements of regulatory bodies, such as the United States Food and Drug Administration (FDA), which require that microbiologic cultures be obtained for approval of anti-infectives for complicated IAI. Nonetheless, there are conditions, such as a peri-appendiceal phlegmon, which are clearly a complicated IAI, but may be managed without a source control procedure and microbiologic analysis.

Patients with complicated IAI may be characterized as manifesting secondary or tertiary peritonitis, single or multiple intra-abdominal abscesses, or an intra-abdominal phlegmon. Secondary peritonitis, arising as a result of perforation of a hollow viscus, is the most straightforward of these terms $[4,13]$. The term secondary peritonitis, however, does not always imply an infection, because peritoneal inflammation may be the result of chemical irritation in the absence of an overt infection, as may be found in patients with a perforated gastroduodenal ulcer. The term also does not convey important information about the extent of the infection-i.e., whether or not it is a localized or diffuse process-nor about the chronicity of the infection, both of which may affect prognosis.

There is much less agreement as to what constitutes tertiary peritonitis. One consensus group defined tertiary peritonitis as that which persisted or recurred more than 48 hours after apparently successful management of secondary peritonitis [14]. Many patients, however, such as those with a recurrent abscess after initial management of perforated appendicitis, could be included under this definition. This would result in a group of patients with a mixed severity of illness rather than a group of patients generally considered to be those most seriously ill as a result of IAI. Instead of 
attempting to specifically define tertiary peritonitis, other authors have developed the concept that there is a continuum between secondary and tertiary peritonitis; thus, describing any specific patient as having secondary versus tertiary peritonitis is somewhat arbitrary, unless the patient is clearly at one of the extremes of the spectrum $[13,15,16]$. Given these variable definitions, it is unlikely that patients described as having tertiary peritonitis in various publications are necessarily comparable. Therefore, recommendations have not been made specifically for patients with tertiary peritonitis, but have been included with the recommendations regarding the most severely ill patients with IAI.

\section{Microbiology of IAI}

The microbiology of IAI varies according to source of the infection and whether or not the patient has been exposed to the healthcare setting; exposure to previous antimicrobial therapy may be of particular importance in this regard [1719]. The microbiology of community-acquired IAI (CA-IAI) has been well characterized. These are generally mixed infections involving a number of enteric micro-organisms. The principal gram-negative micro-organisms cultured from infected abdominal fluid or tissue are Escherichia coli and, to a much lesser extent, other Enterobacteriaceae such as Klebsiella spp. or non-fermenting gram-negative aerobes such as Pseudomonas aeruginosa. Streptococci, primarily of the Streptococcus milleri group, are also isolated frequently; enterococci are identified much less frequently in patients with CA-IAI. Enteric anaerobes, with Bacteroides spp., $B$. fragilis predominating, are also common, although clinical laboratories frequently do not isolate most of anaerobic micro-organisms involved in these infections. Anaerobic micro-organisms are more prevalent for sources of infection in the distal gastrointestinal tract [18-28].

Although the prevalence of these micro-organisms in CAIAI has not changed appreciably over time, susceptibilities of these pathogens to various antibiotics have changed, particularly in certain geographic localities. This is particularly true of Enterobacteriaceae. Globally, there is significant resistance of $E$. coli to ampicillin-sulbactam, as well as to fluoroquinolones. Further, the prevalence of extended spectrum $\beta$-lactamase-producing strains of E. coli and Klebsiella spp. among patients with IAI has been increasing in Latin America, Asia, and parts of Europe. These resistant organisms are increasingly being isolated from patients with CAIAI, and not just from those with risk factors for healthcareassociated/hospital-acquired infection (HA-IAI) [28-31].

In patients with HA-IAI, the types of micro-organisms isolated vary to a much greater extent. Most of the pathogens isolated are still enteric flora, but other micro-organisms, such as staphylococci, are also encountered. The incidence of $E$. coli as a causative pathogen decreases somewhat, whereas the incidence of other Enterobacteriaceae, such as Enterobacter spp., as well as lactose-negative gram-negative bacilli, such as $P$. aeruginosa and Acinetobacter spp., have increased. Aerobic streptococci are found much less commonly, but other grampositive micro-organisms, particularly Enterococcus spp., become much more prevalent, particularly in the postoperative setting. Staphylococci, both coagulase-negative species and Staphylococcus aureus, although still uncommon, are also identified more frequently in patients with HA-IAI than those with CA-IAI. The frequency with which anaerobic micro-organisms are present in these HA-IAI may be somewhat lower than in CA-IAI, although this has been less well documented. Non-bacterial pathogens, particularly yeast such as Candida spp., are encountered more frequently in patients with HA-IAI, particularly if there has been previous exposure to broad-spectrum antibiotics [22-28].

Resistance of the micro-organisms involved in HA-IAI to various antimicrobial agents is quite common. This is particularly notable among patients who received multiple courses of antimicrobial therapy, who are frequently among those described as having tertiary peritonitis. Among the micro-organisms encountered in these patients are various multi-drug-resistant (MDR) gram-negative pathogens, such as Pseudomonas spp. and Acinetobacter spp., resistant grampositive cocci, including vancomycin-resistant Enterococcus spp. (VRE) and methicillin-resistant S. aureus (MRSA), and non-C. albicans spp. [15,32,33].

\section{General management of IAI}

Once the diagnosis is made, initial management of IAI includes physiologic stabilization of the patient using intravenous (IV) fluid therapy and other modalities where warranted, an appropriate intervention to control the source of the problem, and early initiation of antimicrobial therapy directed against the likely pathogenic microbial agents [4,34]. Source control and antimicrobial therapy, however, should be individualized to a given patient based on an assessment of that patient's risk for an adverse outcome. This concept of risk stratification of therapy was introduced in previous guidelines [3-5]. As will be detailed subsequently, this risk is influenced by many different factors, some of which may have greater importance for decisions with regard to specific source control modalities and others with regard to selection of antimicrobial therapy $[3,35]$.

After employment of source control and initiation of antimicrobial therapy, subsequent treatment of the patient with IAI will be based in large part on that patient's response to the therapeutic measures actually employed. Typically, signs and symptoms of infection will abate in patients responding favorably to initial treatment. In such patients, further interventions should be minimized, with early discontinuation of antimicrobial therapy. In contrast, patients who do not exhibit resolution of the signs and symptoms of infection within the first several days of treatment may have an ongoing or recurrent IAI, a secondary infection that is potentially the result of collateral damage from antimicrobial therapy, or a noninfectious source of inflammation. In such patients, directed diagnostic and treatment modalities are warranted and not just an extension or alteration of the current therapeutic regimen.

\section{Recommendations}

\section{Risk assessment}

Q 1.1. What are the important risk factors for treatment failure and death in patients with IAI?

$\mathrm{Q}$ 1.2. How should patient risk be categorized when making decisions regarding management of IAI, including source control and antimicrobial therapy?

Q 1.3. What specific criteria should be used to identify patients with IAI as being at lower or higher risk for treatment failure or death? 
Q 1.4. How should patients be identified as having a HAIAI or otherwise at risk for an IAI because of resistant or opportunistic pathogens?

A large number of investigators have used multivariable analyses to identify factors that predict initial treatment failure or death in patients with IAI. These publications have studied distinct patient subsets and used various definitions of treatment failure [24,26,27,36-74]. Data from individual studies are summarized in Supplementary Table A (see online supplementary material at www.liebertpub.com/overview/ surgical-infections/53/).

Not surprisingly, a wide variety of risk factors have been identified. For clarity, these risk factors have been divided into (1) those related to patient characteristics and physiologic changes associated with the infection, both at the time of presentation and after initial source control; (2) those related to the source and extent of the infection itself, as well as the adequacy and timing of source control; and (3) those related to the presence or likely presence of resistant pathogens, and whether or not initial empiric antimicrobial therapy had activity against the microbial pathogens eventually isolated. It should be emphasized that assignment of a particular clinical finding to one of these categories is somewhat arbitrary, and the categories certainly overlap to some extent. This division of risk factors, however, may have some utility in identifying specific interventions for higher-risk patients. For example, identifying the patient at risk for treatment failure because of the extensive nature of the infection within the abdominal cavity may be relevant to selecting a specific source control intervention, whereas identifying a patient at risk for an infection because of a resistant or opportunistic pathogen may suggest a different approach to antimicrobial therapy. Risk factors identified in these multivariable analyses are summarized in Table 4.

In addition to identifying patients as lower or higher risk according to these criteria, the task force has also chosen to continue the previous approach of stratifying patients as having a CA-IAI or an HA-IAI [2,4,5]. This results in a tripartite division into lower-risk patients with CA-IAI, higherrisk patients with CA-IAI, and patients with HA-IAI. This system then provides a framework for making stratified recommendations without creating undue complexity. Lower-risk patients with CA-IAI, such as those with perforated appendicitis, typically have good outcomes, with very low deaths and a $10 \%-20 \%$ incidence of treatment failure $[26,75,76]$ Outcomes in more severely ill, higher-risk patients with CA-IAI are substantially worse, with higher rates of mortality, organ system dysfunction, and treatment failure [27,38,60,77-79]. Patients with HA-IAI represent a distinct class for purposes of therapeutic recommendations. Outcomes are somewhat variable, depending on what other risk factors are present aside from having a HA-IAI, but are quite poor in a substantial number of these patients [27,38,50,52,56,58,60,77-79].

Previous guidelines suggested certain characteristics to guide patient stratification, but these had not been prospectively validated $[2,4]$. Other attempts have been made to establish evidence-based scoring systems for stratifying patients with IAI according to risk [55]; however, thus far, no system for classifying patients with IAI into different risk categories has gained widespread acceptance.
Definitions of sepsis, severe sepsis, and septic shock provided by the Surviving Sepsis Campaign have generally been well accepted and provide a means of identifying the most severely ill patients with IAI [6]. These definitions have undergone revision recently, and the term "severe sepsis" has been dropped. The new definitions of sepsis and septic shock are thought to correspond to patient groups with an estimated $10 \%$ and $40 \%$ overall death (Table 5) [80]. Thus, the task force recommends that patients with IAI meeting the criteria of severe sepsis or septic shock by the older criteria or of sepsis or septic shock by the newer criteria be considered higher-risk patients.

In various studies, APACHE II scores are frequently identified as the strongest predictors of outcome among all clinical variables collected [26,27,36,38,40,42-46,48,4951,60-62,67,78,79,81]. APACHE II scores reflect both premorbid factors, such as patient age and chronic medical conditions, and acute changes in clinical and laboratory values because of physiologic alterations as a result of the infection. The APACHE II scores, however, require access to a large number of clinical and laboratory variables, making them difficult to calculate in an urgent situation; further, they are subject to inter-rater variability. If these scores are obtained, the task force recommends that patients with a score greater than or equal to 10 be considered at higher risk, because treatment failure rates are at least $20 \%-30 \%$ in this group [45,82].

Many patients with IAI will not meet criteria for sepsis or septic shock and may not be able to be assessed by APACHE II scores. Nonetheless, these patients may have a number of the risk factors for an adverse outcome. Based on the multivariable analyses summarized previously, the task force has recognized advanced age (70 years of age or greater), presence of malignant disease, major compromise of cardiovascular, hepatic, or renal function, and hypoalbuminemia as being the most consistently identified physiologic/phenotypic risk factors predicting an adverse outcome [26,3639,42-44,48-50,55,57,59,61,62,65-67,69]. The task force has suggested that patients having two or more of these risk factors be considered higher-risk patients (Table 6).

Other characteristics related to the abdominal infection itself have also been identified as placing the patient at higher risk for an adverse outcome. The source of the infection has not been a consistent predictor of outcome in multivariable analyses $[26,56,59,60,61,63]$; the task force does not recommend taking this into consideration when stratifying patients for risk. Infections associated with diffuse peritonitis involving all abdominal quadrants, however, have more consistently been associated with an adverse outcome [24,57]. The Mannheim peritonitis index (MPI) includes this as a component, along with several other factors. Although infrequently obtained, an elevated MPI score is also associated with an adverse outcome $[15,44,46,68,69,79,83]$. A delay in source control after IAI is diagnosed $[26,49,63]$ and an inability to achieve adequate initial source control [37,48,51,61,71,84] also correlate highly with an adverse outcome. Based on these findings, the task force has suggested that patients with diffuse peritonitis, an elevated MPI score (if obtained), and those who have delayed or inadequate initial source control be considered higher-risk patients (Table 6).

Patients infected with resistant or opportunistic microorganisms have been identified as being at increased risk for 
Table 4. Risk Factors for Adverse Outcomes with Intra-Abdominal Infection

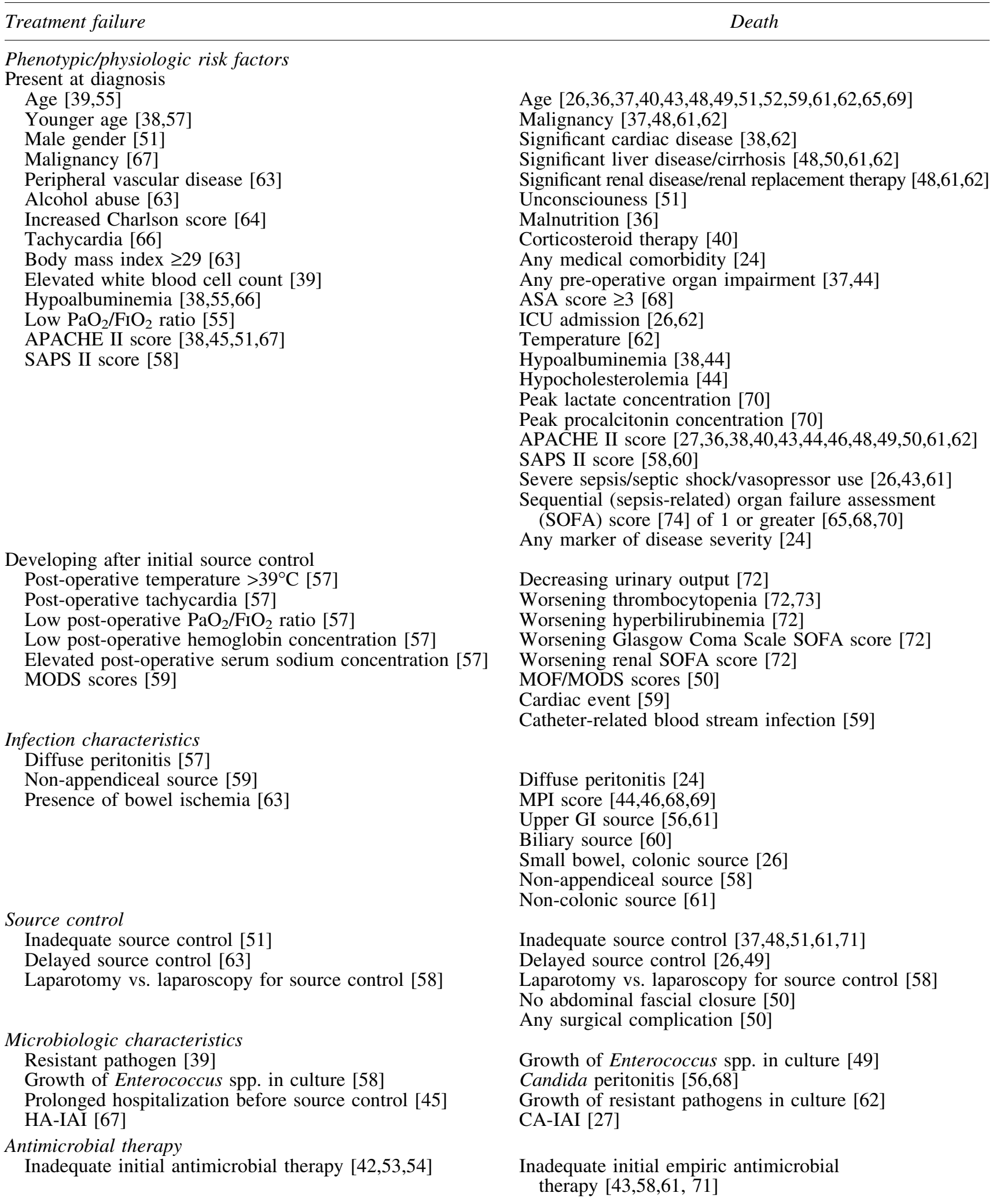

ASA = American Society of Anesthesiologists; ICU = intensive care unit; APACHE = Acute Physiology and Chronic Health Evaluation; SAPS II=Simplified Acute Physiology Score II; MOF=multiple organ failure; MODS=multiple organ dysfunction syndrome; MPI = Mannheim Peritonitis Index; GI = gastrointestinal; HA-IAI = healthcare- or hospital-associated; CA-IAI = community-acquired intraabdominal infection. 
Table 5. Surviving Sepsis Campaign Criteria for Sepsis, Severe Sepsis, and Septic Shock

\section{Criteria [8]}

Sepsis

Documented or suspected infection plus some of the following:

General variables

Fever $\left(>38.3^{\circ} \mathrm{C}\right)$

Hypothermia (core temperature $<36^{\circ} \mathrm{C}$ )

Heart rate $>90 / \mathrm{min}$ or more than two SD above the normal value for age

Tachypnea

Altered mental status

Significant edema or positive fluid balance $(>20 \mathrm{~mL} / \mathrm{kg}$ over $24 \mathrm{~h})$

Hyperglycemia (plasma glucose $>140 \mathrm{mg} / \mathrm{dL}$ or $7.7 \mathrm{mmol} / \mathrm{L}$ ) in the absence of diabetes mellitus

Inflammatory variables

Leukocytosis (WBC count $>12,000 \mu \mathrm{L}$ )

Leukopenia (WBC count $<4,000 \mu \mathrm{L}$ )

Normal WBC count with greater than $10 \%$ immature forms

Plasma C-reactive protein more than two SD above the normal value

Plasma procalcitonin more than two SD above the normal value

Hemodynamic variables

Arterial hypotension (SBP $<90 \mathrm{~mm} \mathrm{Hg}$, MAP $<70 \mathrm{~mm} \mathrm{Hg}$, or a SBP decrease $>40 \mathrm{~mm} \mathrm{Hg}$ in adults

or less than two SD below normal for age)

Organ dysfunction variables

Arterial hypoxemia $\left(\mathrm{PaO}_{2} / \mathrm{FIO}_{2}<300\right)$

Acute oliguria (urine output $<0.5 \mathrm{~mL} / \mathrm{kg} / \mathrm{h}$ for at least $2 \mathrm{~h}$ despite adequate fluid resuscitation)

Creatinine increase $>0.5 \mathrm{mg} / \mathrm{dL}$ or $44.2 \mu \mathrm{mol} / \mathrm{L}$

Coagulation abnormalities (INR $>1.5$ or aPTT $>60 \mathrm{sec}$ )

Ileus (absent bowel sounds)

Thrombocytopenia (platelet count $<100,000 \mu \mathrm{L}^{-1}$ )

Hyperbilirubinemia (plasma total bilirubin $>4 \mathrm{mg} / \mathrm{dL}$ or $70 \mu \mathrm{mol} / \mathrm{L}$ )

Tissue perfusion variables:

Hyperlactatemia $(>1 \mathrm{mmol} / \mathrm{L})$

Decreased capillary refill or mottling

Severe sepsis

Sepsis-induced tissue hypoperfusion or organ dysfunction (any of the following thought to be because of the infection):

Sepsis-induced hypotension

Lactate above upper limits laboratory normal

Urine output $<0.5 \mathrm{~mL} / \mathrm{kg} / \mathrm{h}$ for more than $2 \mathrm{~h}$ despite adequate fluid resuscitation

Acute lung injury with $\mathrm{PaO}_{2} / \mathrm{FIO}_{2}<250$ in the absence of pneumonia as infection source

Acute lung injury with $\mathrm{PaO}_{2} / \mathrm{FIO}_{2}<200$ in the presence of pneumonia as infection source

Creatinine $>2.0 \mathrm{mg} / \mathrm{dL}(176.8 \mu \mathrm{mol} / \mathrm{L})$

Bilirubin $>2 \mathrm{mg} / \mathrm{dL}(34.2 \mu \mathrm{mol} / \mathrm{L})$

Platelet count $<100,000 \mu \mathrm{L}$

Coagulopathy (INR >1.5)

Septic shock

Sepsis-induced hypotension persisting despite adequate fluid resuscitation

\section{Criteria [80]}

Sepsis

Documented or suspected infection plus either:

(1) An acute change in the total SOFA score $\geq 2$ points, or

(2) A qSOFA score of $\geq 2$ points, based on at least two of the following suspected to be because of infection:

Respiratory rate $\geq 22 / \mathrm{min}$

Altered mentation

Systolic blood pressure $\leq 100 \mathrm{~mm} \mathrm{Hg}$

Septic shock

Sepsis with persisting hypotension requiring vasopressors to maintain MAP $\geq 65 \mathrm{~mm} \mathrm{Hg}$ and having a serum lactate level $>2 \mathrm{mmol} / \mathrm{L}(18 \mathrm{mg} / \mathrm{dL})$ despite adequate volume resuscitation.

$\mathrm{SD}=$ standard deviation; $\mathrm{WBC}=$ white blood cell; $\mathrm{SBP}=$ systolic blood pressure; $\mathrm{MAP}=$ mean arterial pressure; INR $=\mathrm{International}$ Normalized Ratio; aPTT = activated partial thromboplastin time; SOFA = Sequential Organ Failure Assessment; qSOFA =quick Sequential Organ Failure Assessment. 
Table 6. Factors Potentially Identifying Patients WITH INTRA-ABDOMINAL INFECTION AT HIGHER RISK

Phenotypic/physiologic risk factors

Advanced age ( $\geq 70 \mathrm{y})$

Malignancy

Significant cardiovascular compromise

Significant liver disease or cirrhosis

Significant renal disease

Hypoalbuminemia

Extent of infection/adequacy of initial source control

Diffuse, generalized peritonitis

Elevated MPI score

Delayed initial source control

Inability to achieve adequate source control

Microbiologic characteristics

Suspected infection with resistant pathogens

MPI = Mannheim Peritonitis Index.

an adverse outcome $[24,39,42,43,45,49,58,62,68]$. There is a broad body of evidence that patients with HA-IAI, including patients with post-operative infections, are at increased risk for infection with resistant or opportunistic pathogens [15,17,22-24,26,32,33,62,77,85-90]. Patients who are not hospitalized, however, may nonetheless be at risk for IAI because of these micro-organisms. Patients at risk for infections with resistant or opportunistic pathogens, including IAI, include those who are known carriers of a resistant organism [91-106] and those who have had recent exposure to broadspectrum antimicrobial therapy [17,19,99,106-123]. The task force would include such patients in the category of patients having HA-IAI (Table 7).

Patients exposed to other healthcare settings outside the hospital may also be at risk of harboring more resistant pathogens. This concept had been applied to patients with healthcare-associated pneumonia, but this concept has recently come into question [124]. Risk factors described previously included hospitalization for greater than 48 hours within the preceding 90 days, residence in a skilled nursing or other long-term care facility, and a history of home infusion therapy, home wound care, or renal replacement therapy within the preceding 30 days [125]. Unfortunately, there are little data to determine whether these risk factors identify patients at risk for IAI because of resistant pathogens. For now, the task force would suggest classifying these patients as potentially having HA-IAI, such that a decision whether or not to direct therapy against resistant pathogens would be considered.

Table 7. Criteria for HealthCare- or HospitalACQUIRED INTRA-ABDOMINAL INFECTION

Infection developing greater than $48 \mathrm{~h}$ after initial source control.

Hospitalized for greater than $48 \mathrm{~h}$ during current admission or within the previous $90 \mathrm{~d}$.

Residence in a skilled nursing or other long-term care facility within the previous $30 \mathrm{~d}$.

Home infusion therapy, home wound care, or dialysis within the preceding $30 \mathrm{~d}$.

Use of broad-spectrum antimicrobial therapy for $5 \mathrm{~d}$ or more during the preceding $90 \mathrm{~d}$.
1.1. We recommend considering phenotypic and physiologic factors, including signs of sepsis or septic shock, extremes of age, and patient co-morbidities; the extent of abdominal infection and adequacy of initial source control; and the presence or persistence of resistant or opportunistic pathogens in assessing risk for treatment failure and death in patients with IAI (Grade 1-B).

1.2. We suggest that patients be characterized as being at either lower or higher risk for treatment failure or death and as having either CA-IAI or HA-IAI (including post-operative infection) for purposes of planning source control and empiric antimicrobial therapy (Grade 2-C).

1.3. We recommend that patients with IAI meeting Surviving Sepsis Campaign criteria for sepsis or septic shock and those having an APACHE II score greater than or equal to 10 be considered higher-risk patients (Grade 1-B). We suggest that patients having at least two physiologic/phenotypic risk factors for an adverse outcome, those having diffuse peritonitis, and those having delayed or inadequate source control also be considered higher-risk patients (Grade 2-B).

1.4. We suggest that patients who have been hospitalized for at least 48 hours during the previous 90 days; those residing in a skilled nursing or long-term care facility during the previous 30 days; those who have received IV infusion therapy, wound care, or renal replacement therapy within the preceding 30 days; those who have received several days of broad-spectrum antimicrobial therapy within the previous 90 days; those who have post-operative infections; and those known to have been colonized or infected previously with a resistant pathogen be considered as having HA-IAI and at risk for infection because of resistant or opportunistic organisms (Grade 2-B).

\section{Source control}

Q 2.1. What is the role of source control in the management of IAI?

Q 2.2. When should the initial source control procedure be undertaken?

Q 2.3. What procedures should be used to obtain source control in patients with IAI?

$\mathrm{Q}$ 2.4. What risk factors identify patients with IAI who are likely to fail initial efforts at source control?

Q 2.5. How should source control be approached in highrisk patients undergoing laparotomy?

Q 2.6. Should higher-risk patients with severe or diffuse peritonitis in whom adequate source control is achieved at the index procedure undergo planned re-laparotomy?

Q 2.7. How should intra-operative lavage be used during source control?

Source control has been summarized as drainage of infected fluid collections, debridement of necrotic infected tissue, and definitive measures to control contamination and restore normal gastrointestinal anatomy and function [34]. Source control should not only reduce bacterial and toxin load by removing the focus of infection and ongoing contamination, but also transform the local environment such that further microbial growth is impeded and host defenses can be optimized [126-130]. Studies of patients with IAI have demonstrated repeatedly that a failure to obtain adequate source control is one of the factors most strongly associated with an adverse outcome, including death (Supplementary Table B; see online supplementary 
material at www.liebertpub.com/overview/surgical-infections/ 53/) $[37,48,51,61,71,84]$. Thus, even though not definitively tested using RCTs, the magnitude of the increase in death and other adverse outcomes associated with inadequate source control makes it clear that these interventions are of prime importance in treating most patients with IAI.

Although expeditious source control is the standard of care for most patients with IAI, certain highly selected patients with a localized IAI have been treated successfully with antiinfective therapy alone. Source control has been omitted or delayed in patients with acute colonic diverticulitis, when the inflammatory process extends only into the peri-colonic tissues [131,132], in patients with perforated appendicitis and a peri-appendiceal phlegmon [133-135], in selected patients with localized upper gastrointestinal perforations [136-138], and in patients with relatively small diverticular or periappendiceal abscesses (generally less than $3-4 \mathrm{~cm}$ in diameter) $[131,132,139-142]$.

There are also some patients for whom a more delayed approach to source control is advocated. For instance, early surgical intervention is associated with increased morbidity and death in patients with infected pancreatic necrosis, and a delay in definitive therapy for days or even weeks may be warranted [128,143-145]. Outside of clinical situations in which there has been well-documented success using a noninterventional approach, however, an expeditious source control procedure is considered essential for patients with IAI. Careful clinical observation is mandatory in patients treated without source control, and patients who fail to improve on antimicrobial therapy alone should undergo a source control intervention $[81,129,131,132]$.

There is sparse evidence regarding the ideal timing of source control interventions. Short delays may be needed to mobilize technical expertise and other resources for optimal treatment of the patient with IAI. Multivariable analyses have identified the time between diagnosis and source control as a predictor of death in patients with IAI $[49,63]$. One reviewer concluded that most patients with peritonitis should undergo a source control procedure within 24 hours of the diagnosis of the infection [146]. Studies of septic patients undergoing source control for IAI and other types of infection, however, suggested that delays of only 3-6 hours were associated with increased death $[147,148]$. Based on these limited data, the recommendations found in previous guidelines and reviews, and the opinions of the members of this task force, most patients with IAI should undergo source control within 24 hours; patients with sepsis or septic shock should undergo earlier source control, although a short delay to allow for rapid resuscitation may be needed in the hemodynamically unstable patient with sepsis $[4,6,49,90,128,146]$.

Selection of a specific source control procedure for a given patient should be predicated both on the characteristics of the infection and the patient, as well as the availability of technical expertise at the local institution. Operative and other invasive procedures present additional stresses to patients whose physiologic reserves have already been challenged by the infection, so limiting the extent of the source control procedure may be advantageous under some circumstances. If source control is inadequate, however, persistence of the infectious insult may result in an adverse outcome. The balance between the competing demands of optimally managing the infection and ameliorating the ef- fects of source control on the patient's physiologic function requires individualized patient assessment and careful clinical judgment [128].

The utility of less invasive source control interventions has been confirmed for many patients with IAI. A robust body of evidence has shown that percutaneous drainage of infected intra-abdominal fluid collections provides adequate source control [128,149]. Success rates range from 82\%-91\% in various series using these techniques [150-153]. In general, minimally invasive approaches are reasonable for most patients with localized IAI, when feasible. The efficacy of less invasive approaches for patients with more diffuse IAI is uncertain, however. Although case series had demonstrated good outcomes using laparoscopic drainage without colonic resection for the treatment of selected patients with Hinchey class III or IV diverticulitis [154-157], recent prospective trials suggested that this approach was less successful than colectomy $[158,159]$. Overall, based on the available evidence, the task force concluded that the least invasive intervention that will fulfill the goal of establishing adequate source control should be used preferentially in most patients with IAI. Unless clearly shown to be effective, however, less invasive approaches may not be warranted in patients with diffuse peritonitis. This echoes the recommendations from the previous guideline and those of other authors [4, $6,128,149]$.

The identification of certain risk factors for an adverse outcome in patients with IAI might be useful in selecting alternative approaches to source control interventions. Higherrisk patients, particularly those identified as having an acute compromise of their physiologic status, are a group of patients who might benefit from such alternative approaches [4, 6,128]. Patients with diffuse peritonitis, identified directly or through an elevated MPI score, are another such group of patients $[15,24,44,46,51,57,68,69,79,81,83]$; however, the utility of such observations in modifying approaches to source control remains conjectural for the most part [160].

The use of abbreviated or damage control laparotomy with delayed fascial closure is an approach that has been used to treat severely ill patients with IAI $[161,162]$. There are observational data supporting the use of this approach in certain patients. One such group of patients are those whose physiologic reserves are exhausted, as manifested by parameters such as $\mathrm{pH}$ less than 7.2, a temperature less than $35^{\circ} \mathrm{C}$, or clinically evident coagulopathy [162-164]. The use of open abdominal techniques to manage or prevent abdominal compartment syndrome is also widely accepted [163-165]; this syndrome may occur in patients who have IAI with sepsis and have received large amounts of IV fluids for resuscitation. An inability to obtain full source control at the time of the index procedure is another indication for use of damage control laparotomy and temporary abdominal closure to facilitate early re-laparotomy for more definitive source control [149,162-164,166-169]. Patients with bowel ischemia may also benefit from abbreviated laparotomy and temporary abdominal closure to facilitate planned second-look procedures [162,163,166,168,169],

The use of planned or mandatory re-laparotomy had been suggested as an approach for treating all patients with severe, diffuse secondary peritonitis. A RCT comparing mandatory planned re-laparotomy to re-laparotomy based on clinical indications in patients with severe secondary peritonitis, 
however, showed no significant benefit of planned relaparotomy in terms of peritonitis-related morbidity or death (Supplementary Table C; see online supplementary material at www.liebertpub.com/overview/surgical-infections/53/); moreover, resource utilization was higher in patients undergoing mandatory re-laparotomy [170,171]. Additional, nonrandomized studies have also questioned the need for planned re-laparotomy in patients with severe peritonitis when adequate source control can be achieved [149,170,172-177]. Based on these data, the task force concluded that damage control laparotomy may be useful in selected patients, but that neither this approach nor mandatory re-laparotomy should be applied universally to patients with severe, diffuse peritonitis.

Irrigation or lavage of the peritoneal cavity is widely used in the management of IAI. A review of the available data concluded that there was no overall benefit for peritoneal lavage beyond that necessary for removal of gross contamination [178]. Several more recent series and one prospective trial of patients with perforated appendicitis likewise have found that aspiration and limited irrigation to remove gross contamination were as effective as lavage [179-182]. The use of continuous post-operative lavage in patients with peritonitis has not been supported by reviews of the available evidence $[178,183]$. The inclusion of antibiotic agents in lavage fluid has also been used in an effort to reduce complications related to IAI. A meta-analysis of seven older studies of diverse patient populations, not all of whom had IAI, suggested that the inclusion of antibiotic agents in lavage fluid reduced post-operative septic complications, but had no effect on death. Because of the poor methodology used in these studies, however, the authors of this meta-analysis did not believe that the data supported the efficacy of antibiotic lavage, and that this remained an open question [178].

The precise definition of what constitutes adequate source control remains elusive [126]. Approaches to source control have changed as new techniques develop and evidence accumulates regarding various management options. Even when detailed descriptions of adequate source control are provided, interobserver variation in assessing source control adequacy is quite high $[184,185]$. Local variations in practice patterns may also lead to different, but not necessarily inappropriate, approaches to source control [185], Given the complexity of defining adequate source control, the task force does not believe that assessments of source control adequacy in patients with IAI would be a useful performance measure by which to evaluate surgical competence.

2.1. We recommend routine use of a source control procedure to remove infected fluid and tissue and to prevent ongoing contamination in patients with IAI except for those patients with clinical problems for which clear evidence has shown that a non-interventional approach is associated with a good clinical outcome (Grade 1-A).

2.2. We suggest that source control be undertaken within 24 hours of the diagnosis of IAI, except for those infections for which clinical evidence indicates non-interventional or delayed management is appropriate (Grade 2-B). We suggest that patients with sepsis or septic shock undergo source control in a more urgent manner (Grade 2-C).

2.3. We recommend use of the least invasive approach that is able to achieve adequate source control, at least on a temporary basis, for patients with IAI (Grade 1-B).
2.4. We suggest that patients with major physiologic instability, those with diffuse infections, and those with ongoing bowel ischemia be considered at higher risk for failure of initial source control and for use of alternative or temporizing approaches to source control (Grade 2-B).

2.5. We recommend that an abbreviated laparotomy and temporary abdominal closure techniques be used in critically ill patients with IAI if closure of the abdomen would create meaningful intra-abdominal hypertension, if the patient's physiologic reserves are severely compromised, if there is an inability to achieve adequate source control with the initial procedure, or if there is a plan for a second-look laparotomy because of mesenteric ischemia (Grade 1-B).

2.6. We recommend against routine planned relaparotomy in high-risk patients with severe peritonitis when adequate source control can be obtained at the time of the index procedure; such patients should be treated with ondemand rather than scheduled re-laparotomy (Grade 1-B).

2.7. We suggest use of irrigation with crystalloid fluid to remove visible debris and gross contamination before abdominal closure in patients with IAI, generally limiting lavage to those areas with gross involvement as an adjunct to the source control procedure (Grade 2-B).

\section{Microbiologic evaluation}

Q 3.1. Should cultures be obtained in lower-risk patients with CA-IAI?

Q 3.2. Should cultures be obtained in higher-risk patients with CA-IAI and those with HA-IAI?

Q 3.3. Should routine peritoneal cultures be obtained from patients with IAI for epidemiologic purposes?

The value of routine culture data in guiding antimicrobial therapy for lower-risk patients with CA-IAI has not been rigorously evaluated in prospective trials [186]. Nevertheless, a number of observational studies have suggested that these cultures rarely, if ever, provide information useful to the clinician [187-191]. The majority of lower-risk patients with CA-IAI are successfully treated with standard approaches to source control and antimicrobial therapy. When treatment failure is attributed to inadequate antimicrobial therapy in such patients, it is generally because the initial empiric regimen lacked activity against the common Enterobacteriaceae or enteric anaerobes responsible for the infection [54,187,192]. Nonetheless, the increased prevalence of extended-spectrum $\beta$-lactamase (ESBL)-producing Enterobacteriaceae strains in certain geographic areas may necessitate a modification of the recommendation against obtaining routine cultures, particularly if this phenomenon leads to a substantial increase in treatment failure in lower-risk patients with CA-IAI.

There is a greater rationale for obtaining routine peritoneal cultures in higher-risk patients with CA-IAI and those with HA-IAI. In patients with sepsis, use of appropriate antimicrobial therapy is considered essential [8]; inadequate empiric antimicrobial therapy has been associated with higher deaths in patients with sepsis, including those with IAI [43,54,58,187,193-195]. Higher-risk patients with CA-IAI may be infected with somewhat more resistant pathogens than lower-risk patients [22-24,26,27], and the likelihood of encountering resistant pathogens is substantially higher in patients with HA-IAI [15,17,22-24,26,32,33,62, $77,85,87,90]$. 
To provide adequate antimicrobial therapy to higher-risk patients, use of broad-spectrum empiric antimicrobial regimens is recommended [4,6,13,127]. To avoid excessive exposure of the patient to broad-spectrum antimicrobial therapy and potential selection of further resistant microorganisms, however, de-escalation of therapy based on culture results is also recommended [4, 6,196-198]. In addition to deescalating antimicrobial therapy, culture and susceptibility results can be used to guide pathogen-directed therapy when an unexpected or resistant pathogen is encountered; even though such therapy may be delayed, it is still more likely to be successful than continued administration of an inappropriate empiric regimen [197,199,200].

There have been substantial increases in resistance of microbial pathogens involved in IAI to previously effective antimicrobial agents, particularly in Asia and Latin America [2831]. Ideally, epidemiologic investigations could identify problematic pathogens in a specific geographic locale, and the results could be used to guide selection of preferred empiric antimicrobial regimens for that region [186]. To track these epidemiologic changes, culture results from an unbiased sample of patients must be obtained. Thus, culture and susceptibility data from even lower-risk patients with CA-IAI are needed, although the culture results themselves will provide little direct benefit to the patient. For such an approach to be viable, however, adequate resources are needed for aggregation, analysis, and dissemination of this information. Given the increasing incidence of resistant pathogens in both CA-IAI and HA-IAI and the limited antimicrobial armamentarium available to treat patients with such micro-organisms, such investigations will likely be increasingly vital in the future.

Optimal techniques for obtaining peritoneal cultures were reviewed in the previous guideline [4]. At least one $\mathrm{mL}$ of peritoneal fluid or infected tissue should be collected and placed in a suitable transport system for examination by the microbiologic laboratory. If anaerobic cultures are to be obtained, these specimens need to be sent in an anaerobic transport system. Direct inoculation of fluid specimens into blood culture bottles is an additional option. In any case, use of peritoneal swabs instead of fluid or tissue for cultures is strongly discouraged.

3.1. We do not recommend routinely obtaining peritoneal fluid cultures in lower-risk patients with CA-IAI for purposes of guiding antimicrobial therapy (Grade 1-B).

3.2. We recommend obtaining cultures of peritoneal fluid in higher-risk patients with CA-IAI and in patients with HAIAI to identify potential resistant or opportunistic pathogens (Grade 1-C).

3.3. We suggest obtaining cultures in all patients with CA-IAI and HA-IAI if adequate resources are available to aggregate and analyze the epidemiologic data and the information can be used to guide empiric antimicrobial therapy (Grade 2-C).

\section{Intravenous antimicrobial agents}

\section{A. General principles}

Q 4.1. What are the general principles regarding antimicrobial therapy for patients with IAI?

Standard antimicrobial therapy for patients with IAI should include agents with activity against aerobic gram-negative Enterobacteriaceae, aerobic streptococci, and obligate enteric an- aerobic organisms found in the gastrointestinal tract, although coverage of the latter may not be absolutely essential in patients with an upper gastrointestinal source of infection. Additional antimicrobial agents, providing coverage of less common resistant or opportunistic pathogens, may be warranted in more severely ill patients. A wide variety of antimicrobial agents have activity against these various micro-organisms. This section summarizes the task force's interpretation of the utility of various antimicrobial agents in treating patients with IAI. A synopsis of these recommendations is available in Table 8. Summaries of older RCTs evaluating antimicrobial therapy for IAI are included in the previous evidence review [3]; summaries of more recent RCTs are provided in the Supplementary Tables (see online supplementary material at www.liebertpub.com/ overview/surgical-infections/53/).

The basic principle of providing empiric antimicrobial therapy effective against gram-negative Enterobacteriaceae, aerobic streptococci, and obligate enteric anaerobic organisms in patients with IAI was described several decades ago and has been a component of all previous guidelines [1,2,4,5]. Observational studies have demonstrated an increased risk of treatment failure and death when this basic principle is not followed (Supplementary Table D; (see online supplementary material at www.liebertpub.com/overview/surgicalinfections/53/) [54,58,187,201,202]. A recent large database study of more than 6000 patients confirmed that patients who received regimens lacking activity against anaerobic organisms had a significantly increased rate of treatment failure compared with patients who received agents effective against both gram-negative Enterobacteriaceae and anaerobic pathogens (Supplementary Table D) [192].

4.1. We recommend use of antimicrobial regimens having activity against the typical gram-negative Enterobacteriaceae, gram-positive cocci, and obligate anaerobes involved in these infections (Grade 1-A).

\section{B. Aminoglycoside-based regimens}

$\mathrm{Q}$ 4.2. What is the role of aminoglycoside-based regimens in the treatment of patients with IAI?

At one time, aminoglycosides (gentamicin, tobramycin, amikacin, netilmicin), in combination with an anti-anaerobic agent (clindamycin, metronidazole) and variably with an agent active against gram-positive organisms, were considered the "gold standard" for treatment of patients with IAI. These regimens were recommended in early guidelines [1,2], but not in subsequent guidelines [4,5]. Aminoglycosides were used in four RCTs published since 2000 (Supplementary Table E (see online supplementary material at www.liebertpub.com/ overview/surgical-infections/53/)). In one, the clinical efficacy of amikacin, in combination with ceftazidime and metronidazole, was found to be inferior to cefoperazone-sulbactam [203]. No significant differences in outcome were noted in the other three studies; however, one only compared once daily to multiple daily doses of amikacin [204-206].

Two meta-analyses published since 2000 found that aminoglycoside-based regimens were inferior to comparators for treating patients with IAI (Supplementary Table F; see online supplementary material at www.liebertpub.com/ overview/surgical-infections/53/) [207,208]. In the previous evidence review [3], four trials also showed higher treatment 


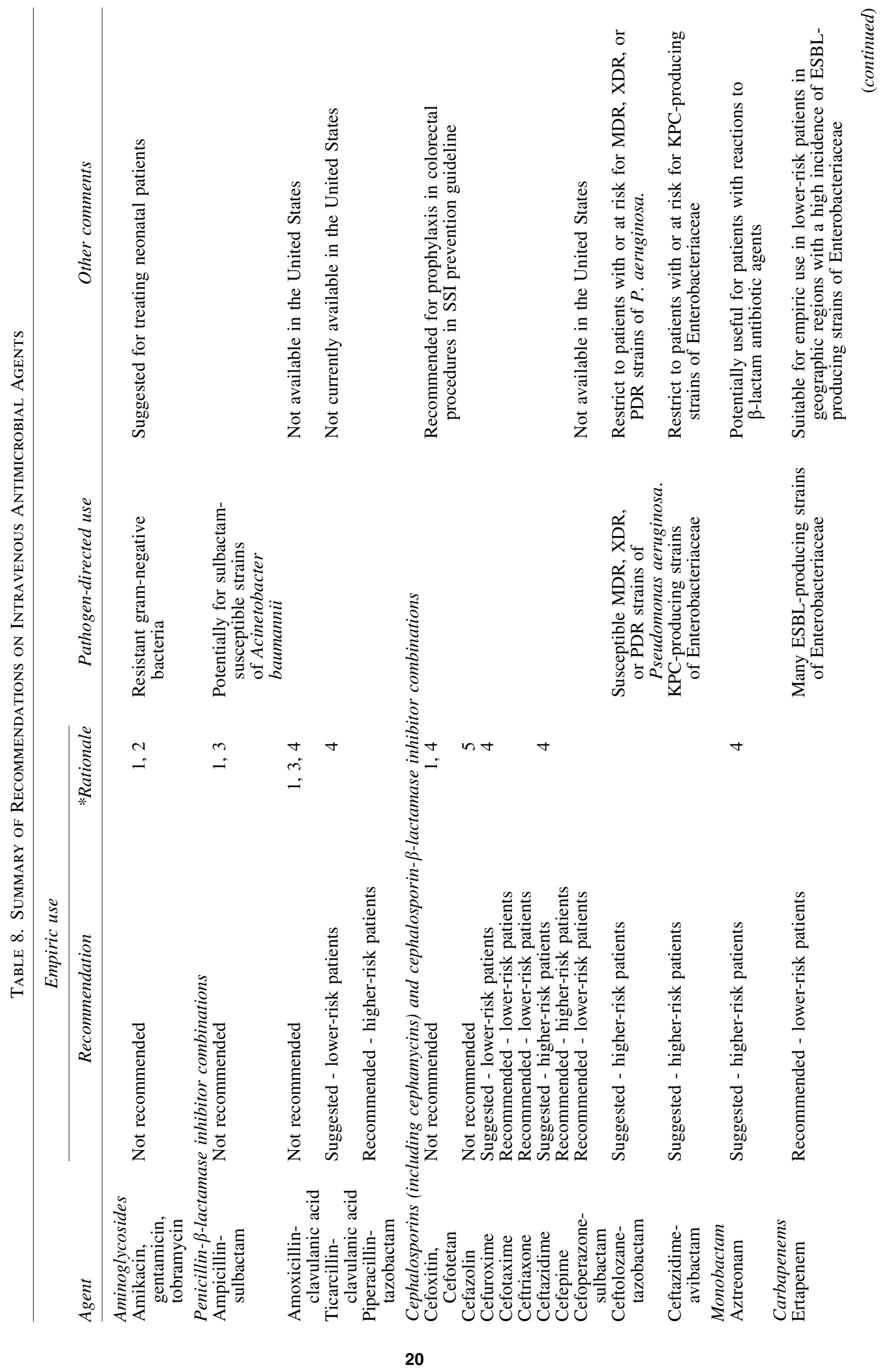




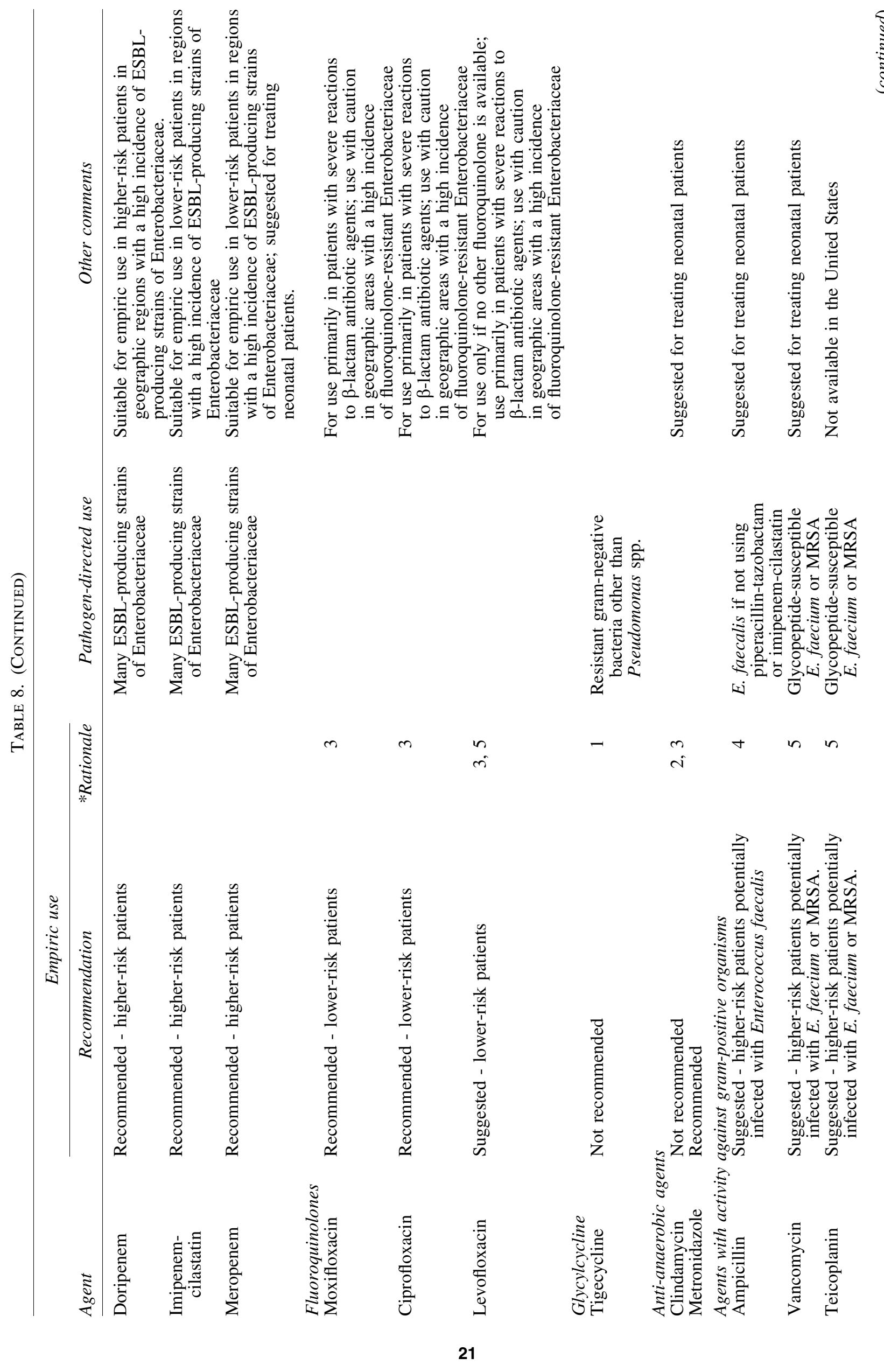




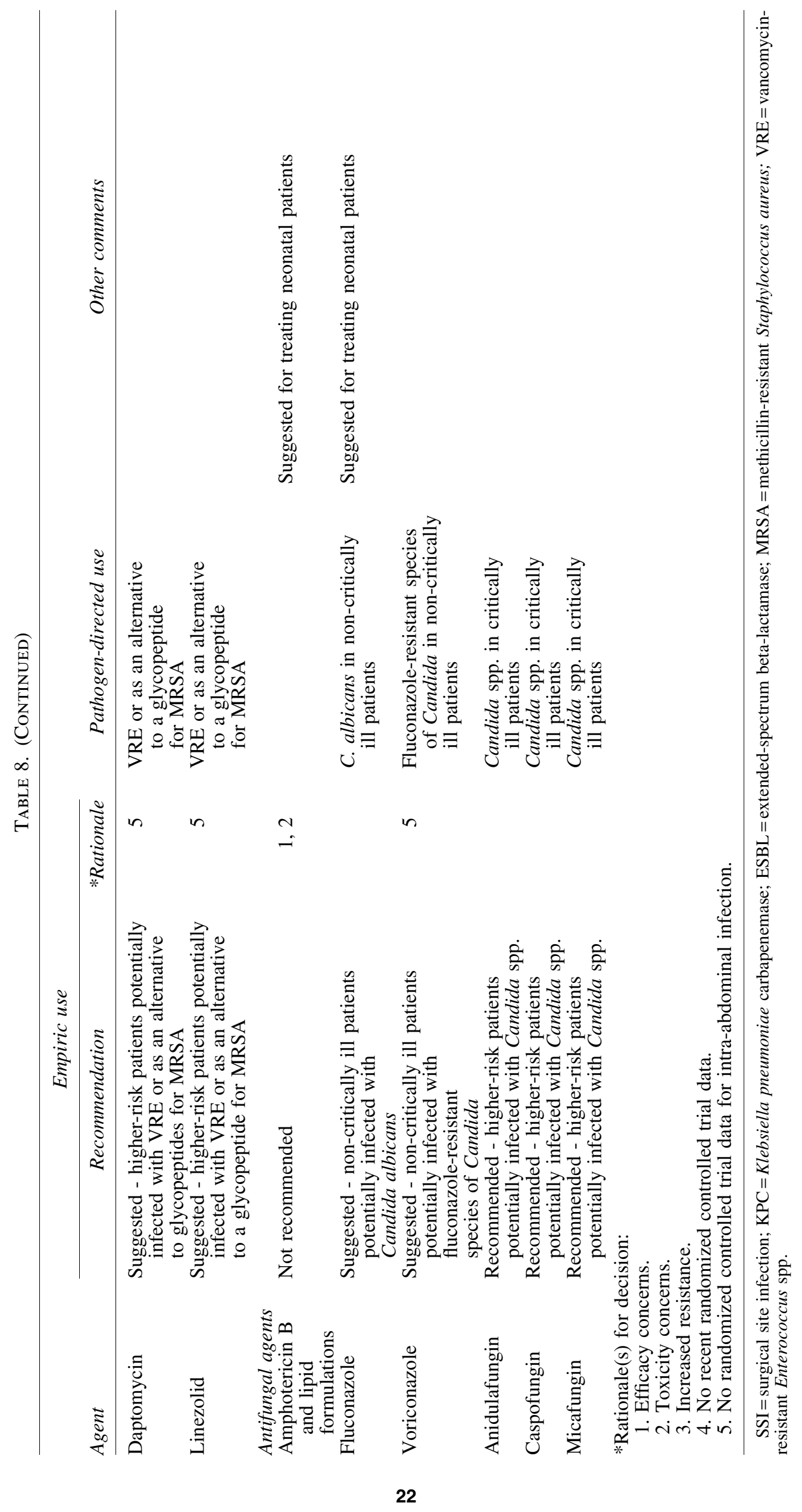


failure rates with aminoglycoside-based regimens compared with other agents [209-212], whereas one trial demonstrated superiority of an aminoglycoside-based regimen compared with ampicillin-sulbactam [213]. The nephrotoxicity and ototoxicity of these agents as well as the need for therapeutic drug monitoring further complicates their use. Given the availability of alternative agents, the task force concluded that these agents should not be used routinely for empiric therapy of patients with IAI. These agents may be useful, however, for treating patients with an infection because of a resistant gram-negative pathogen for which other classes of agents are not suitable. Unfortunately, many of these microorganisms are also resistant to aminoglycosides [28]. Aminoglycoside-based regimens are still used when treating neonatal patients with IAI, as will be discussed in Section 13.

4.2. We recommend against the routine use of aminoglycoside-based regimens for the empiric treatment of patients with IAI (Grade 1-B). We suggest that these regimens may be useful for treatment of IAI in neonatal patients and in adults and children because of resistant gram-negative organisms, if other agents are not suitable (Grade 2-B).

\section{Penicillin- $\beta$-lactamase inhibitor combinations}

Q 4.3a. What is the role of ampicillin-sulbactam in the treatment of patients with IAI?

Q 4.3b. What is the role of IV amoxicillin-clavulanic acid in the treatment of patients with IAI?

Q 4.3c. What is the role of ticarcillin-clavulanic acid in the treatment of patients with IAI?

Q 4.3d. What is the role of piperacillin-tazobactam in the treatment of patients with IAI?

Ampicillin-sulbactam was recommended for treatment of patients with IAI in an older guideline [2], but not in the more recent guidelines [4,5]. The RCTs evaluating the efficacy of ampicillin-sulbactam for the treatment of IAI are limited. Since 2008, two studies have been published using ampicillinsulbactam (Supplementary Table G; see online supplementary material at www.liebertpub.com/overview/surgical-infections/ 53/) [214,215], one of which found ampicillin-sulbactam inferior to ertapenem [214]. Three older articles were previously reviewed [3], one of which found ampicillin-sulbactam inferior to a regimen of gentamicin plus clindamycin [213]. Microbiologic data have documented substantial resistance of gramnegative Enterobacteriaceae globally to ampicillin-sulbactam; in the most recent Study for Monitoring of Antimicrobial Resistance Trends (SMART) data, only 34\% of hospitalassociated strains and $45 \%$ of community-acquired strains of E. coli were susceptible to this agent [28].

Based on the clinical and microbiologic data, the task force concluded that other agents were preferable to ampicillinsulbactam for the empiric treatment of patients with IAI. Ampicillin-sulbactam does have some activity against $A$. baumannii, primarily because of the sulbactam entity, and may have some use in pathogen-directed therapy of patients with infections from that micro-organism [216]; however, the majority of Acinetobacter strains isolated from patients with IAI are not susceptible [28].

The IV preparation of amoxicillin-clavulanic acid is not available for use in the United States but is available in Europe and other parts of the world. The literature search did not identify any RCTs published since 2000 evaluating this agent. In the previous evidence review [3], one small prospective trial comparing use of IV amoxicillin-clavulanic acid plus metronidazole to IV ciprofloxacin plus metronidazole found lower success rates with the amoxicillinclavulanic acid plus metronidazole regimen, although this difference was not statistically significant [217]. In a recent observational study of patients with peritonitis in The Netherlands, the authors concluded that amoxicillin-clavulanic acid-resistant $E$. coli was increasingly common, and that use of this agent in such patients was associated with increased death [218].

Microbiologic studies suggest that in vitro resistance of $E$. coli to amoxicillin-clavulanic acid is high; in one study, susceptibility of $E$. coli isolates from various sources to amoxicillin-clavulanic acid was $50 \%-51 \%$, which was only marginally better than that of ampicillin-sulbactam $(45 \%-$ $47 \%$ ) [219]. Because of concerns regarding efficacy and resistance of $E$. coli as well as the lack of recent RCTs on use of this agent, the task force does not support use of amoxicillinclavulanic acid for empiric treatment of patients with IAI.

Ticarcillin-clavulanic acid had been recommended for empiric treatment of patients with IAI in previous guidelines $[1,2,4,5]$. Since 2000 , only one small RCT has evaluated this agent (Supplementary Table G; see online supplementary material at www.liebertpub.com/overview/surgical-infections/ 53/). No significant differences in success rates were demonstrated comparing use of this agent with ertapenem, but only 11 clinically evaluable patients were treated with ticarcillin-clavulanic acid [220]. In the previous evidence review [3], three prospective trials demonstrated efficacy of ticarcillin-clavulanate for the treatment of lower-risk patients with IAI. Contemporary data on the activity of this agent against gram-negative pathogens found with IAI are limited. Although the activity spectrum of ticarcillin-clavulanic acid includes $P$. aeruginosa, in vitro susceptibility has declined in recent years [221]. Ticarcillin-clavulanic acid may have some activity against MDR organisms such as A. baumannii, although less than ampicillin/sulbactam [222], and some activity against ESBL-producing strains of Enterobacteriaceae, but less than piperacillin-tazobactam [223]. Ticarcillin-clavulanic acid is no longer available in the United States [224]. The task force concluded that this agent was acceptable for empiric treatment of patients with IAI, although other agents would be preferable because of the lack of contemporary data demonstrating efficacy of this agent. This recommendation is moot, however, unless production of it resumes.

Piperacillin-tazobactam is a commonly used agent for treating patients with IAI. It has been recommended for the treatment of higher-risk patients or those with higher severity infections in previous guidelines [2,4,5]. Six RCTs comparing this agent with other regimens for IAI have been published since 2000 (Supplementary Table G; see online supplementary material at www.liebertpub.com/overview/ surgical-infections/53/) [225-230], one of which was reviewed previously [3]. There were no significant differences noted in the clinical success rates between patients receiving piperacillin-tazobactam and those receiving various comparators. Eight other trials evaluating this agent were reviewed previously [3], including one in severely ill patients comparing use of piperacillin-tazobactam with the combination of this agent plus an aminoglycoside. It was also concluded that 
piperacillin-tazobactam was as effective as the various comparators [3]. In vitro susceptibility data generally document good activity of this agent against $E$. coli, although there has been some decline in this over the past decade. Piperacillintazobactam is active against many ESBL-producing Enterobacteriaceae but generally less so than carbapenems [28].

Based on the continued evidence of good efficacy of piperacillin-tazobactam, the task force recommends this agent for the treatment of patients with IAI. To avoid excessive use and potential promotion of resistance to broadspectrum agents such as piperacillin-tazobactam, the task force suggests that this agent be reserved primarily for use in higher-risk patients.

4.3a. We suggest that ampicillin-sulbactam not be used for the empiric treatment of adults and children with IAI (Grade $2-B)$.

4.3b. We suggest that IV amoxicillin-clavulanic acid not be used for the empiric treatment of adults and children with IAI (Grade 2-B).

4.3c. We suggest that ticarcillin-clavulanic acid is an acceptable agent for the empiric treatment of lower-risk adults and children greater than one month of age with CA-IAI, if it is available (Grade 2-B).

4.3d. We recommend piperacillin-tazobactam as an acceptable agent for the empiric treatment of adults and children older than one month with IAI (Grade 1-A). We suggest that this agent be reserved primarily for higher-risk patients because of its broader-spectrum antimicrobial activity (Grade 2-C).

D. Cephalosporin-based regimens and cephalosporin- $\beta$ lactamase inhibitor combinations

Q 4.4a. What is the role of cephamycin antibiotic agents (cefoxitin, cefotetan) in the treatment of patients with IAI? $\mathrm{Q}$ 4.4b. What is the role of cefazolin plus metronidazole in the treatment of patients with IAI?

Q 4.4c. What is the role of cefuroxime plus metronidazole in the treatment of patients with IAI?

Q 4.4d. What is the role of cefotaxime or ceftriaxone plus metronidazole in the treatment of patients with IAI?

$\mathrm{Q} 4.4 \mathrm{e}$. What is the role of ceftazidime plus metronidazole in the treatment of patients with IAI?

Q 4.4f. What is the role of cefepime plus metronidazole in the treatment of patients with IAI?

$\mathrm{Q} 4.4 \mathrm{~g}$. What is the role of cefoperazone-sulbactam in the treatment of patients with IAI?

Q 4.4h. What is the role of ceftolozane-tazobactam plus metronidazole in the treatment of patients with IAI?

Q 4.4i. What is the role of ceftazidime-avibactam plus metronidazole in the treatment of patients with IAI?

Cephamycin antibiotic agents have been recommended variably in previous guidelines as monotherapy for the treatment of patients with IAI $[1,2,4,5]$. The literature search did not identify any RCTs published since 2000 that evaluated the use of these agents. In the previous evidence review [3], eight trials evaluated cefoxitin in patients with IAI, the most recent published in 1996, and three trials evaluated cefotetan, the most recent published in 1994. None of these demonstrated significant differences in clinical outcomes comparing cephamycins with other agents.
Because of the lack of contemporary data on the use of these agents, recent studies analyzing use of these antibiotic agents for surgical prophylaxis in colorectal procedures were also reviewed. One RCT found cefotetan inferior to ertapenem for this indication [231]. Two recent, large database studies found that cephamycins were less efficacious than other recommended agents for prophylaxis for colorectal procedures [232,233]. Microbiologic data indicate that about $90 \%$ of E. coli strains isolated from patients with IAI in North America are susceptible to cefoxitin [234], but this is lower in other parts of the world $[31,235]$. Resistance of anaerobic micro-organisms, including $B$. fragilis, to cephamycins is increasing, however [236,237]. A concern specific to cefoxitin is its short half-life of $0.7-1.1$ hours. Because of this, intra-operative re-dosing every two hours is recommended for surgical site infection prophylaxis [238].

Overall, given the lack of contemporary data and the potential for decreased efficacy of cephamycin antibiotic agents, as reflected in the indirect evidence from studies of colorectal surgical procedure prophylaxis, the task force suggests use of regimens other than cephamycins for empiric treatment of patients with IAI. It is recognized that this conflicts with a recent guideline on surgical prophylaxis, which recommends cephamycins for appendectomy and colorectal procedures [238]. Nevertheless, because there are alternative regimens for lower-risk patients with CA-IAI, the task force believes that these should be used preferentially to cephamycins.

Unlike other cephalosporins, cephamycins have in vitro activity against many ESBL-producing Enterobacteriaceae. This suggests their potential utility in treating those resistant pathogens; however, there are virtually no data evaluating their clinical efficacy for this indication [239].

The combination of cefazolin plus metronidazole was included as a recommended regimen in some previous guidelines [4,5]. Cefazolin has been approved by the FDA for the treatment of patients with biliary tract disease, but not for management of complicated IAI. There are essentially no prospective data evaluating the efficacy of this regimen compared with others for IAI. One trial from 1989 studied this regimen in patients with complicated appendicitis but did not compare it with an alternative regimen [240]. There are two large database studies that evaluated antibiotic agents for colorectal prophylaxis, both of which suggested cefazolin plus metronidazole was efficacious for this indication $[232,233]$. Relatively recent data show that $92 \%$ of $E$. coli strains from patients with acute and perforated appendicitis are susceptible to cefazolin [241]. Nevertheless, because there is only indirect evidence supporting the use of cefazolin plus metronidazole for treatment of patients with IAI, the task force suggests using alternative regimens for empiric therapy.

The combination of cefuroxime plus metronidazole was also recommended in previous guidelines for the treatment of patients with IAI $[2,4,5]$. This recommendation was supported by three trials showing efficacy of this regimen, which were included in the previous evidence review [3]. There are no recent trials re-evaluating this regimen for IAI and relatively few recent microbiologic studies. A study of appendiceal isolates demonstrated $95 \%$ susceptibility of E. coli to cefuroxime [241], but a global survey of urinary isolates found only $82 \%$ susceptibility of E. coli to this agent [242]. The task force concluded that this regimen was acceptable for 
empiric treatment of lower-risk patients with CA-IAI but suggested preferential use of other regimens for which more recent evidence is available.

The combination of a third-generation cephalosporin plus an anti-anaerobic agent has been recommended in all previous guidelines $[1,2,4,5]$; the 2010 guideline specifically recommended use of cefotaxime or ceftriaxone plus metronidazole for lower-risk patients with CA-IAI [4]. Cefotaxime plus metronidazole was compared with piperacillintazobactam in one study published since 2000 (Supplementary Table $\mathrm{H}$; see online supplementary material at www. liebertpub.com/overview/surgical-infections/53/); this study demonstrated similar outcomes with either regimen [225]. The previous evidence review [3] included five studies comparing cefotaxime plus an anti-anaerobic agent with other agents. In one of these studies, success rates were significantly higher with cefotaxime plus metronidazole than with meropenem [243], whereas the opposite was true in a second study [244]. In one additional small study comparing a regimen of cefotaxime plus gentamicin plus metronidazole with ciprofloxacin plus metronidazole, the fluoroquinolonebased regimen appeared to be more efficacious [245].

Nine studies have been published since 2000 comparing ceftriaxone and a nitroimidazole (generally metronidazole) with other agents (Supplementary Table H; see online supplementary material at www.liebertpub.com/overview/ surgical-infections/53/) [204,246-253]. Three additional trials were cited in the previous evidence review [3]. None of the more recent studies demonstrated any significant difference in outcomes between patients receiving ceftriaxone plus a nitroimidazole and those receiving the comparator; one of the older studies found ceftriaxone plus metronidazole to be superior to an aminoglycoside-based regimen.

Approximately $90 \%$ of $E$. coli obtained from patients with IAI in North America are susceptible to cefotaxime or ceftriaxone. Susceptibility rates are appreciably lower in other parts of the world, however, particularly Asia, the Middle East, and Latin America, where there is a high prevalence of ESBL-producing Enterobacteriaceae [28,31,234,235]. Based on the evidence available, the task force recommends use of cefotaxime or ceftriaxone plus metronidazole for the treatment of lower-risk patients with CA-IAI. In areas of the world where ESBL-producing $E$. coli are prevalent, however, other agents would be preferable.

Previous guidelines recommended the combination of the third-generation anti-pseudomonal cephalosporin, ceftazidime, plus metronidazole for the treatment of higher-risk patients with IAI $[2,4,5]$. The task force did not identify any recent RCTs evaluating ceftazidime plus metronidazole only, although one recent trial compared ceftazidime plus amikacin and metronidazole with cefoperazone/sulbactam monotherapy (Supplementary Table H; see online supplementary material at www.liebertpub.com/overview/surgical-infections/ 53/); this study, performed in a region where there is a high incidence of ESBL-producing E. coli, found greater efficacy with cefoperazone-sulbactam than the ceftazidime-based regimen [203]. In the previous review [3], two studies compared ceftazidime plus an anti-anaerobic agent to other agents; one of these demonstrated superiority of ceftazidime plus metronidazole over an aminoglycoside plus metronidazole [210]. In vitro susceptibilities of $E$. coli to ceftazidime remain greater than $90 \%$ in North America and Europe; however, the susceptibility of this organism to ceftazidime is appreciably lower in Asia, the Middle East, and Latin America, where ESBL-producing strains of E. coli are prevalent [28,31,234,235].

Based on the available data, the task force suggests that ceftazidime plus metronidazole can be used for the treatment of patients with IAI, although this is graded as a weak recommendation because of the lack of contemporary data. As with most other agents with broad-spectrum activity against gram-negative organisms, this regimen should be reserved primarily for empiric treatment of higher-risk patients with IAI. A ceftazidime-based regimen should most likely be avoided in regions where there is a high prevalence of ESBLproducing Enterobacteriaceae. In general, the task force prefers use of cefepime rather than ceftazidime for those patients in whom an anti-pseudomonal cephalosporin is believed warranted.

The fourth-generation anti-pseudomonal cephalosporin, cefepime, in combination with metronidazole, was also recommended for the treatment of higher-risk patients with IAI in previous guidelines $[2,4,5]$. Cefepime plus metronidazole was evaluated in two RCTs published since 2000 (Supplementary Table $\mathrm{H}$; see online supplementary material at www .liebertpub.com/overview/surgical-infections/53/) [254,255] and in two RCTs included in the previous evidence review [3]. Two of these trials demonstrated significantly higher success rates with cefepime plus metronidazole compared with imipenem-cilastatin [45,255], although this statistical difference disappeared in one study when the results were adjusted for an imbalance in the severity of illness between the study groups [45]. As with ceftazidime, microbiologic data indicate in vitro susceptibility of $E$. coli to cefepime is greater than $90 \%$ in North America and Europe but appreciably lower in Asia, the Middle East, and Latin America [28,31,234,235]. Cefepime is less susceptible to hydrolysis by AmpC $\beta$-lactamases than are other broad-spectrum cephalosporins, so it may be a therapeutic alternative to carbapenems for patients with infections because of gram-negative bacteria expressing those enzymes [256-259].

Overall, based on the available data, the task force recommends cefepime plus metronidazole for the treatment of patients with IAI. The task force suggests that this regimen be used primarily for empiric therapy of higher-risk patients because of its potent anti-pseudomonal activity.

Several combinations of a cephalosporin with a $\beta$ lactamase inhibitor are now available that may be able to overcome some the resistance mediated by ESBL enzymes. Cefoperazone-sulbactam is one such combination. This agent is not available in the United States. In one recent trial, cefoperazone-sulbactam was superior to a regimen of amikacin, ceftazidime, and metronidazole for the treatment of patients with IAI (Supplementary Table $\mathrm{H}$; see online supplementary material at www.liebertpub.com/overview/ surgical-infections/53/); of note, this trial took place in a region with a high prevalence of ESBL-producing Enterobacteriaceae [203]. Two additional trials using cefoperazonesulbactam were described in the previous evidence review [3], one of which found cefoperazone-sulbactam superior to an aminoglycoside-based regimen [209].

Cefoperazone-sulbactam has activity against a number of ESBL-producing strains of E. coli, although this varies somewhat from country to country [260-262]. This agent has 
been tested primarily in lower-risk patients with IAI. Overall, the task force concluded that cefoperazone-sulbactam is an effective agent for the treatment patients with IAI and may be of value for use in lower-risk patients with CA-IAI who live in parts of the world where the prevalence of ESBLproducing Enterobacteriaceae is appreciable.

Two new cephalosporin- $\beta$-lactamase inhibitor combinations, ceftolozane-tazobactam and ceftazidime-avibactam, used in combination with metronidazole, have been approved by the FDA for the treatment of patients with complicated IAI. These agents have also now been approved for use by the European Commission. In phase II and phase III trials, ceftolozane-tazobactam, in combination with metronidazole, appeared to have similar efficacy to meropenem for the treatment of patients with IAI (Supplementary Table H; see online supplementary material at www.liebertpub.com/ overview/surgical-infections/53/) [263,264]. Ceftolozanetazobactam is active in vitro against many ESBL-producing Enterobacteriaceae, although not against $K$. pneumoniae carbapenemase (KPC)-producing strains or metallo- $\beta$ lactamase (MBL)-producing strains. It is also active against many strains of $P$. aeruginosa and appears to be the most potent currently available $\beta$-lactam or $\beta$-lactam- $\beta$-lactamase inhibitor combination against this organism [265-268]. Of particular note is the substantial activity of ceftolozanetazobactam against many MDR and extremely drug resistant (XDR) strains of $P$. aeruginosa, including strains that are resistant to ceftazidime and carbapenems [269-273].

Ceftazidime-avibactam also has an expanded, albeit somewhat different spectrum of activity against gramnegative micro-organisms. As with ceftolozane-tazobactam, the clinical efficacy of ceftazidime-avibactam in combination with metronidazole was compared with meropenem for the treatment of patients with IAI in phase II and phase III trials (Supplementary Table H; see online supplementary material at www.liebertpub.com/overview/surgical-infections/53/). Success rates in patients treated with this agent were noninferior to those observed in patients treated with meropenem [274,275]. Ceftazidime-avibactam has activity against most strains of Enterobacteriaceae, including ESBL-producing strains and AmpC $\beta$-lactamase-producing strains. Ceftazidimeavibactam is the only currently available $\beta$-lactam- $\beta$ lactamase inhibitor combination with substantial in vitro activity against KPC-producing Enterobacteriaceae but is not active against MBL-producing micro-organisms [268,276278]. Ceftazidime-avibactam also has good in vitro activity against $P$. aeruginosa, including a number of MDR and XDR strains [277,279].

Based on the clinical and microbiologic data, the task force concluded that both ceftolozane-tazobactam plus metronidazole and ceftazidime-avibactam plus metronidazole are acceptable regimens for the treatment of patients with IAI. Of some concern, however, was the finding that both agents were less effective than meropenem in subgroups of patients with renal impairment $[275,280]$. Further research will be needed to clarify the role of these agents in the treatment of patients with IAI. For now, the task force suggests that these regimens be reserved for the treatment of higher-risk patients with CAIAI or HA-IAI who are either known or strongly suspected of being infected with one of the resistant pathogens that are uniquely susceptible to one of these agents, when other antibiotics are not suitable. 4.4a. We suggest that cefoxitin and cefotetan not be used for the empiric treatment of adults and children with IAI (Grade 2-B).

4.4b. We suggest that cefazolin plus metronidazole not be used for the empiric treatment of adults and children with IAI (Grade 2-C).

4.4c. We suggest that cefuroxime plus metronidazole is an acceptable regimen for the empiric treatment of lower-risk adults and children older than one month with CA-IAI (Grade 2-B).

4.4d. We recommend cefotaxime or ceftriaxone plus metronidazole for the empiric treatment of lower-risk adults and children older than one month with CA-IAI (Grade 1-A).

4.4e. We suggest that ceftazidime plus metronidazole is an acceptable agent for the empiric treatment of adults and children older than one month with IAI (Grade 2-A). We suggest that this regimen be reserved primarily for higherrisk patients because of its broader-spectrum antimicrobial activity (Grade 2-C).

4.4f. We recommend cefepime plus metronidazole for the empiric treatment of adults and children older than one month with IAI (Grade 1-A). We suggest that this regimen be reserved primarily for higher-risk patients because of its broader-spectrum antimicrobial activity (Grade 2-C).

4.4g. We suggest that cefoperazone-sulbactam, where available, is an acceptable agent for the empiric treatment of lower-risk adults and children older than one month with CAIAI (Grade 2-B).

4.4h. We suggest that ceftolozane-tazobactam plus metronidazole is an acceptable regimen for the empiric treatment of adults with IAI (Grade 2-A). Because of the unique spectrum of activity of ceftolozane-tazobactam against certain ESBL-producing Enterobacteriaceae and against resistant strains of $\mathrm{P}$. aeruginosa, we suggest this regimen be used primarily for selected patients with IAI strongly suspected or proven to be caused by one of those resistant pathogens, for whom other agents are not suitable (Grade 2-C).

4.4i. We suggest that ceftazidime/avibactam plus metronidazole is an acceptable regimen for the empiric treatment of adults with IAI (Grade 2-A). Because of the unique spectrum of activity of ceftazidime-avibactam against many ESBL-producing Enterobacteriaceae, particularly KPCproducing Enterobacteriaceae, we suggest this regimen be used primarily for selected patients with IAI strongly suspected or proven to be caused by one of those resistant pathogens, for whom other agents are not suitable (Grade 2-C).

\section{E. Aztreonam-based regimen}

$\mathrm{Q}$ 4.5. What is the role of an aztreonam-based regimen in the treatment of patients with IAI?

Previous guidelines recommended an aztreonam-based regimen for the treatment of patients with IAI $[1,2,4,7]$. There have been no RCTs published since 2000 evaluating use of this agent for the treatment of such patients. The previous evidence review [3] identified three RCTs evaluating aztreonam plus clindamycin for the treatment of patients with IAI, all of which demonstrated non-inferiority of this regimen. Aztreonam has no anti-anaerobic activity and is almost devoid of activity against gram-positive organisms [281]. The current and some previous guidelines recommend use of metronidazole rather than clindamycin as the preferred anti-anaerobic agent when 
combination regimens are needed. If metronidazole is used instead of clindamycin in conjunction with aztreonam, however, the regimen lacks activity against most gram-positive cocci. Therefore, for use in patients with IAI, aztreonam should be combined with metronidazole and with an agent that has activity against aerobic gram-positive cocci, such as vancomycin.

Although aztreonam has good in vitro activity against many gram-negative bacteria, it has relatively poor activity against ESBL-producing strains of $E$. coli and $K$. pneumoniae [282]. Aztreonam appears to be safe for use in patients with serious allergic reactions to other $\beta$-lactams [283]; thus, an aztreonam-based regimen may be of utility in such patients when other $\beta$-lactam agents cannot be used.

Overall, the task force believes that the regimen of aztreonam in combination with metronidazole and vancomycin is acceptable for treatment of patients with IAI, particularly those patients with serious $\beta$-lactam allergies for whom other alternatives may not be suitable. Use of alternative agents would be preferable, however, when such an option is available, because of the lack of contemporary data on the efficacy of this combination regimen. The task force also suggests that this aztreonam-based regimen be reserved primarily for higher-risk patients, because of its broad-spectrum activity against gram-negative pathogens.

4.5. We suggest that the regimen of aztreonam plus metronidazole plus vancomycin is acceptable for the empiric treatment of adults and children older than one month with IAI (Grade 2-B). We suggest this regimen be reserved primarily for higher-risk patients, particularly those with serious $\beta$-lactam allergies, because of its broader-spectrum activity against gram-negative pathogens (Grade 2-C).

\section{F. Carbapenem}

Q 4.6a. What is the role of ertapenem in the treatment of patients with IAI?

Q 4.6b. What is the role of the broad-spectrum carbapenem, doripenem, in the treatment of patients with IAI?

$\mathrm{Q}$ 4.6c. What is the role of the broad-spectrum carbapenems, imipenem-cilastatin, and meropenem, in the treatment of patients with IAI?

Ertapenem was been recommended for the treatment of patients with IAI in previous guidelines $[2,4,5]$. The task force identified nine RCTs published since 2000 evaluating use of this agent as monotherapy for IAI (Supplementary Table I; see online supplementary material at www.liebertpub.com/ overview/surgical-infections/53/) [205,214,220,226,228,230, $246,247,284]$, one of which [226] was reviewed before publication in the previous evidence review [3]. Success rates with ertapenem have been similar to those of comparators except in one trial, in which ertapenem was found to be superior to ampicillin-sulbactam [214]. Ertapenem is active against the most common strains of ESBL-producing Enterobacteriaceae but is not active against KPC-producing and MBL-producing strains [265,285]. Increased use of carbapenems may lead to selection of gram-negative pathogens expressing these resistance genes. Thus far, little decline in the susceptibilities of intraabdominal isolates of $E$. coli to carbapenems has been detected, but there have been some decrease in $K$ pneumoniae susceptibilities [28]. Ertapenem does not have appreciable antibacterial activity against Enterococcus spp. or P. aeruginosa [286,287].
The task force concluded that ertapenem is an acceptable agent for monotherapy for lower-risk patients with CA-IAI. Because its spectrum of activity is narrower than that of other carbapenems, the task force recommends use of broaderspectrum carbapenems for empiric treatment of higher-risk patients with CA-IAI and those with HA-IAI, although treatment could be de-escalated to ertapenem if resistant pathogens were not identified in definitive cultures. Ertapenem is also a good option for treating patients in areas of the world where there is a high prevalence of ESBL-producing E. coli in the community.

The broad-spectrum carbapenems-doripenem, imipenemcilastatin, and meropenem-have been recommended for the treatment of patients with IAI in previous guidelines as they have become available $[1,2,4,5]$. Doripenem was compared with meropenem for the treatment of adult patients with IAI in two large RCTs (Supplementary Table I; see online supplementary material at www.liebertpub.com/overview/ surgical-infections/53/) [288,289]. No significant differences were observed in clinical success rates between the two carbapenems. An aborted clinical trial was also reported comparing doripenem with meropenem in pediatric patients with IAI, nearly all of whom had perforated appendicitis [290]; both agents appeared to be effective in the limited number of patients completing this trial before it was terminated. Doripenem, however, has not been approved for use in pediatric patients [291].

Imipenem-cilastatin has been evaluated in seven RCTs published since 2000 (Supplementary Table I; see online supplementary material at www.liebertpub.com/overview/ surgical-infections/53/) [227,255,292-296] and in 19 RCTs cited in the previous evidence review [3]. In one small recent study, success rates in patients treated with imipenemcilastatin were significantly lower than in patients treated with cefepime plus metronidazole [255]. Among studies reviewed previously, one found imipenem-cilastatin superior to comparators [211]; however, two found imipenemcilastatin inferior to comparators [45,297], although a higher severity of illness in patients receiving imipenem-cilastatin may have accounted for the decreased efficacy observed in one trial [45].

Meropenem has also been evaluated extensively in RCTs, including eight published since 2000 (Supplementary Table I; see online supplementary material at www.liebertpub.com/ overview/surgical-infections/53/) $\quad[263,264,274,275,288-$ $290,292]$. There were no statistically significant differences in outcomes between meropenem and comparators in these recent trials. In 11 RCTs reviewed previously [3], two found significant differences between meropenem and comparators, with one favoring meropenem and one favoring the comparator [243,244].

As with ertapenem, the broad-spectrum carbapenems generally retain activity against the common ESBL-producing strains of gram-negative bacteria, but not against KPC- or MBL-producing strains [286,287]. Increased resistance to broad-spectrum carbapenems has been observed for some gram-negative microorganisms, however, particularly $P$. aeruginosa and $A$. baumannii, which may be a reflection of increased reliance on carbapenems in areas of high prevalence of ESBL-producing bacteria [298]. Based on the available data, the task force recommends the use of doripenem, imipenem-cilastatin, or meropenem for the 
treatment of adult patients with IAI, and imipenem-cilastatin or meropenem for the treatment of pediatric patients with IAI. Because of their broad-spectrum activity, their use as empiric therapy should be restricted to higher-risk patients with CAIAI or HA-IAI.

4.6a. We recommend ertapenem for the empiric treatment of lower-risk adults and children older than one month with CA-IAI (Grade 1-A).

4.6b. We recommend doripenem for the empiric treatment of adults with IAI (Grade 1-A). We do not recommend the use of doripenem for empiric treatment of children older than one month with IAI unless no other options are available (Grade 1-C). We suggest that this agent be reserved primarily for higher-risk patients because of its broader-spectrum antimicrobial activity (Grade 2-C).

4.6c. We recommend imipenem-cilastatin and meropenem for the empiric treatment of adults and children older than one month with IAI (Grade 1-A). We suggest that these agents be reserved for higher-risk patients because of their broaderspectrum antimicrobial activity (Grade 2-C).

\section{G. Fluoroquinolones and fluoroquinolone-based regimens}

$\mathrm{Q}$ 4.7a. What is the role of moxifloxacin in the treatment of patients with IAI?

Q 4.7b. What is the role of ciprofloxacin plus metronidazole in the treatment of patients with IAI?

Q 4.7c. What is the role of levofloxacin plus metronidazole in the treatment of patients with IAI?

The previous guideline recommended moxifloxacin monotherapy for the treatment of patients with IAI [4]. Moxifloxacin has been evaluated in five RCTs published since 2000 (Supplementary Table J; see online supplementary material at www.liebertpub.com/overview/surgicalinfections/53/) [215,229,250,251,284]. No significant differences were observed in success rates using moxifloxacin compared with other agents in any of these trials. A metaanalysis also found moxifloxacin monotherapy to be noninferior to treatment with comparators (Supplementary Table K; see online supplementary material at www. liebertpub.com/overview/surgical-infections/53/) [299]. Moxifloxacin has not been evaluated in pediatric patients. Concerns have been raised about resistance of anaerobic micro-organisms to moxifloxacin, but a recent analysis showed no correlation between anaerobic susceptibility data and clinical outcome with use of moxifloxacin [300]. Similarly, the decreased susceptibility of E. coli and other gram-negative organisms to ciprofloxacin and levofloxacin [28] would be expected to apply to moxifloxacin as well. These decreases in susceptibility, however, have not led to obvious increases in failure rates in recent clinical trials.

Based on the available data, the task force still recommends use of moxifloxacin as monotherapy for lower-risk patients with CA-IAI, particularly for patients who have serious or life-threatening reactions to $\beta$-lactam antibiotic agents. Other agents would be preferable, however, if there is an appreciable prevalence of fluoroquinolone-resistant E. coli in the local environment.

The regimen of ciprofloxacin plus metronidazole was recommended for use in patients with IAI in previous guidelines [2.4.5]. Two RCTs evaluating ciprofloxacin plus metronidazole have been published since 2000 (Supplementary Table J; see online supplementary material at www. liebertpub.com/overview/surgical-infections/53/) [247,249] these are in addition to four studies included in the previous evidence review [3]. Success rates using ciprofloxacin plus metronidazole have been equivalent to comparators in all of these studies, with two of the older studies reporting a significantly higher success rate with this regimen [245,301]. A meta-analysis of RCTs reported higher success rates with use of ciprofloxacin plus metronidazole than comparators for treatment of patients with IAI (Supplementary Table K; see online supplementary material at www.liebertpub.com/ overview/surgical-infections/53/) [302]. Although ciprofloxacin has been approved for certain pediatric indications, concern has been expressed about potential side effects in pediatric patients. Recent reviews have concluded that this agent is relatively safe to use in children [303,304]. The increasing world-wide resistance of $E$. coli to ciprofloxacin is also of concern [28], although it has not been clearly associated with decreased clinical responses to fluoroquinolones in patients with IAI.

Based on all data, the task force concludes that ciprofloxacin plus metronidazole is a reasonable option for the treatment of lower-risk patients with CA-IAI. In geographic localities with a high prevalence of fluoroquinolone-resistant E. coli, however, a non-fluoroquinolone-based regimen would be preferable. For higher-risk patients, the task force believes that ciprofloxacin should be used primarily in a pathogen-directed fashion, only if peritoneal cultures reveal susceptible gram-negative micro-organisms. As with other fluoroquinolones, use of ciprofloxacin plus metronidazole may be an appropriate option in patients who cannot receive $\beta$-lactam antibiotic agents.

Levofloxacin plus metronidazole has been used for the treatment of patients with IAI, but its use has not been reported in any published RCT. Levofloxacin has not received FDA approval for treatment of patients with IAI. Based on its spectrum of activity, it would be expected to have efficacy similar to that of other recommended fluoroquinolones. Similar to ciprofloxacin, levofloxacin has been approved by the FDA for certain pediatric indications. As with other fluoroquinolones, resistance of E.coli to levofloxacin is increasing worldwide [28]. Because levofloxacin may be the sole fluoroquinolone on many hospital formularies, the task force has included levofloxacin plus metronidazole as an acceptable regimen for the treatment of lower-risk patients with CA-IAI. Because of the lack of data documenting its efficacy for treating patients with IAI, however, its use should be limited to situations in which patients cannot be treated with alternative agents, and levofloxacin is the only fluoroquinolone available because of formulary restrictions.

4.7a. We recommend moxifloxacin as an acceptable agent for the empiric treatment of lower-risk adults with CA-IAI, although it should be used with caution in areas of the world where there is a high incidence of fluoroquinolone-resistant E. coli (Grade 1-A). We do not recommend the use of moxifloxacin for empiric treatment of children with IAI unless no other options are available (Grade 1-C).

4.7b. We recommend ciprofloxacin plus metronidazole as an acceptable regimen for the empiric treatment of lower-risk adults with CA-IAI, although it should be used with caution in 
areas of the world where there is a high incidence of fluoroquinolone-resistant E. coli (Grade 1-A). We suggest that ciprofloxacin plus metronidazole may be used for empiric treatment of children older than one month CA-IAI, if other options are not suitable (Grade 2-B).

4.7c. We suggest that levofloxacin plus metronidazole is an acceptable regimen for the empiric treatment of lower-risk adults with CA-IAI, if use of a fluoroquinolone is warranted and it is the only fluoroquinolone available for use (Grade 2C). We suggest that levofloxacin plus metronidazole may be used for empiric treatment of children older than one month with IAI, if other options are not suitable (Grade 2-C).

\section{H. Tigecycline}

Q 4.8. What is the role of tigecycline in the treatment of patients with IAI?

Tigecycline is a glycylcycline that is less susceptible to some of the common mechanisms producing resistance of bacteria to tetracyclines [305]. It was recommended for empiric use in lower-risk patients with CA-IAI in the previous guideline [4]. Use of tigecycline in the treatment of patients with IAI has been described in five RCTs published since 2000 (Supplementary Table L; see online supplementary material at www.liebertpub.com/overview/surgical-infections/53/) [252, 253,294-296]. None of these demonstrated any statistically significant difference in success rates between tigecycline and other agents. In meta-analyses of these RCTs, there was a trend toward lower clinical success rates with tigecycline, which did not reach statistical significance (Supplementary Table M; see online supplementary material at www.liebertpub .com/overview/surgical-infections/53/) [306,307]. Aggregated data from clinical trials using tigecycline for any indication, however, revealed higher deaths in tigecycline-treated patients, which was significant in some of the analyses [306-310]. These data resulted in a black box warning from the FDA indicating that tigecycline should be reserved for situations in which other agents are not suitable.

Tigecycline has in vitro activity against many ESBLproducing strains of Enterobacteriaceae and A. baumannii, although not against $P$. aeruginosa $[311,312]$. In observational studies, tigecycline has been successful in the treatment of patients with IAI likely to harbor resistant micro-organisms [313-315]; however, tigecycline was frequently administered in conjunction with other effective agents in these studies, so its efficacy as a single agent remains uncertain [316-318]. Tigecycline could also offer a carbapenem-sparing option for the treatment of ESBL-producing strains of Enterobacteriaceae.

Overall, the task force concluded that the possibility of lower efficacy and higher deaths argued against a continued recommendation for the routine use of tigecycline as an empiric agent to treat patients with IAI. There are clinical scenarios in which pathogen-directed use of tigecycline for the management of resistant bacteria could be an option, particularly in patients who cannot be treated with other agents, however.

4.8a. We do not recommend tigecycline for the empiric treatment of patients with IAI under most circumstances (Grade 1-B). We suggest that tigecycline may be of use in the treatment of adult patients with resistant pathogens, particularly as a component of a combination regimen, when other agents are not suitable (Grade 2-B).

\section{Anti-anaerobic agents}

Q 4.9a. What is the role of metronidazole as an antianaerobic agent in combined regimens for the treatment of patients with IAI?

Q 4.9b. What is the role of clindamycin as an antianaerobic agent in combined regimens for the treatment of patients with IAI?

Metronidazole is commonly used for the treatment of patients with IAI to provide anti-anaerobic activity, if the agent being used to treat gram-negative micro-organisms lacks such activity. Nearly all recent RCTs have used metronidazole as the anti-anaerobic agent in combination regimens [203-205,225,246,247,249-255,263,264,274,275,319]. Metronidazole was also used extensively in studies reviewed previously [3]. There are no current data and only limited older data from RCTs comparing the efficacy of metronidazole with other anti-anaerobic agents; however, the breadth of studies using this agent attests to its efficacy. The development of major resistance of anaerobic micro-organisms to metronidazole has yet to be observed [236]. Overall, the task force concluded that metronidazole remains the preferred agent for management of anaerobic bacteria when combination regimens are used for the treatment of patients with IAI.

The use of clindamycin as an anti-anaerobic agent to treat patients with IAI has fallen into disfavor. Since 2000, only three RCTs using clindamycin have been published [204,206,320], all in pediatric patients with perforated appendicitis. Clindamycin was used much more frequently in studies published before that date [3]. Use of clindamycin for treatment of patients with IAI has been curtailed because of increased in vitro resistance of anaerobic bacteria, particularly $B$. fragilis, to this agent $[236,321]$ and the heightened concern for the development of Clostridium difficileassociated disease after exposure to clindamycin [322,323]. Based on these considerations and the availability of alternative agents, the task force considers clindamycin to be a second-line anti-anaerobic agent in combination regimens, although it is an option if metronidazole cannot be used.

4.9a. We recommend metronidazole as the preferred antianaerobic agent to be used in combination regimens for the empiric treatment of IAI in adults and children older than one month (Grade 1-B).

4.9b. We suggest that clindamycin not be used as an antianaerobic agent in combination regimens for the empiric treatment of IAI in adults and children older than one month (Grade 2-B) unless other agents cannot be used. We suggest use of this agent is acceptable in children under one month of age (Grade 2-C).

\section{J. Anti-enterococcal and anti-staphylococcal agents}

Q 4.10a. What is the role of ampicillin in the treatment of patients with IAI?

$\mathrm{Q} 4.10 \mathrm{~b}$. What is the role of vancomycin in the treatment of patients with IAI?

$Q$ 4.10c. What are the roles of linezolid and daptomycin in the treatment of patients with IAI?

Treatment of Enterococcus spp. should be considered in higher-risk patients with CA-IAI and those with HA-IAI. Nearly all strains of E. faecalis, including some strains of 
vancomycin-resistant $E$. faecalis, are susceptible to ampicillin $[89,324]$. This agent is used frequently for the treatment of patients with serious infections because of E. faecalis, such as endocarditis or bacteremia [325,326]. Ampicillin has been used frequently also as a component of combination ("triple") antibiotic therapy for IAI, particularly in pediatric patients, although its necessity for that has not been demonstrated [204,327,328]. The task force has concluded that ampicillin is an acceptable agent for managing a proven or suspected IAI because of E. faecalis in higher-risk patients, if the selected regimen lacks activity against that micro-organism.

In IAI, E. faecium is increasingly encountered as a pathogen, particularly in patients with HA-IAI. In contrast to $E$. faecalis, nearly all strains of E. faecium are resistant to ampicillin [89,324,329-332]. Although the incidence of VRE is increasing, vancomycin-susceptible strains of E. faecium can be treated with vancomycin or other glycopeptides such as teicoplanin [324,329,331,332]. The efficacy of empiric vancomycin or teicoplanin as anti-enterococcal agents in patients with IAI has not been evaluated definitively. Broad experience has been gained with use of glycopeptides to manage serious enterococcal infections, however. Therefore, the task force considers vancomycin (or teicoplanin where available) to be the first-line agent for treating most patients with HAIAI proven or suspected to be because of vancomycinsusceptible strains of E. faecium.

Vancomycin should not be used as empiric antienterococcal therapy in patients known to be infected or considered to be at high risk for infection with VRE. Ampicillin may be an option for patients with IAI from vancomycinresistant strains of $E$. faecalis, although susceptibilities should be monitored [333,334]. For patients with vancomycinresistant E. faecium, linezolid or daptomycin are the preferred agents. Both linezolid and daptomycin have good in vitro activity against vancomycin-resistant $E$. faecium [322,329,333,335], and linezolid has been approved by the FDA for the treatment of patients with infections from this resistant pathogen. Observational studies have reported that both agents have been used successfully to treat patients with various types of infections because of VRE, including HAIAI [115,334-337]. Recent meta-analyses of patients with VRE bacteremia suggested lower mortality rates after treatment with linezolid rather than daptomycin; however, the component studies used in these analyses were all retrospective in nature [338,339]. Because of the lack of prospective data, the task force did not attempt to indicate a preference between linezolid and daptomycin for the treatment of IAI from VRE.

Although relatively uncommon, MRSA strains are encountered in patients with HA-IAI, particularly those with post-operative HA-IAI [23,24,26,62,340-342]. There are no published studies specifically evaluating antimicrobial therapy for the treatment of patients with IAI involving MRSA. For patients with serious infections from MRSA, a glycopeptide is frequently described as the preferred agent [334,343-345]. Thus, vancomycin or teicoplanin (where available) would be appropriate choices for empiric therapy of HA-IAI when MRSA is a suspected or proven pathogen. Concerns have been expressed, however, about the efficacy of glycopeptides for managing MRSA infections [345-349] and the nephrotoxicity of these agents [350-352]. Among other agents with activity against MRSA [345,353-355], the task force considered linezolid and daptomycin to be the most reasonable alternatives. Linezolid is at least as effective as vancomycin for the management of infections from MRSA, with some data suggesting superiority [344,348,355-358]. There are some observational data describing successful use of linezolid to treat patients with IAI from MRSA [336]. Daptomycin, likewise, appears to be equivalent to vancomycin for the management of serious MRSA infections [337,354,359] and could be used to treat patients with IAI from MRSA.

4.10a. We suggest that ampicillin may be used for treatment of IAI in adults and children to provide pathogen-directed therapy against susceptible enterococcal strains (Grade 2-B).

4.10b. We suggest that vancomycin may be used for treatment of IAI in adults and children to provide empiric or pathogen-directed therapy for suspected or proven infections from vancomycin-susceptible E. faecium or methicillinresistant $S$. aureus (MRSA) (Grade 2-B). We suggest inclusion of vancomycin in an aztreonam-based regimen for coverage of gram-positive organisms (Grade 2-B).

4.10c. We suggest that linezolid and daptomycin may be used for management of IAI in adults and children to provide empiric or pathogen-directed therapy for suspected or proven infections from vancomycin-resistant Enterococcus spp., and as an alternative to vancomycin for suspected or proven infections because of MRSA (Grade 2-B).

\section{K. Antifungal agents}

Q 4.11a. What is the role of amphotericin B and its lipid formulations in the treatment of patients with IAI?

$\mathrm{Q}$ 4.11b. What is the role of fluconazole in the treatment of patients with IAI?

$\mathrm{Q}$ 4.11c. What is the role of voriconazole in the treatment of patients with IAI?

Q 4.11d. What is the role of echinocandins (anidulafungin, caspofungin, micafungin) in the treatment of patients with IAI?

The antifungal agents most commonly used to treat patients with HA-IAI because of Candida spp. include the polyene amphotericin B and its various formulations; azoles, including fluconazole and voriconazole; and echinocandins, including anidulafungin, caspofungin, and micafungin. Most RCTs evaluating these agents have enrolled primarily patients with candidemia and only limited numbers of patients with candidal IAI. In a number of these RCTs, outcomes of these latter patients have not been reported separately.

Use of amphotericin B has fallen into disfavor because of toxicity, despite the development of alternative formulations designed to combat that problem [360]. Two RCTs published since 2000 reported lower success rates in small numbers of adult patients with intra-abdominal candidiasis treated with either amphotericin B or liposomal amphotericin B versus those treated with caspofungin or micafungin (Supplementary Table N; see online supplementary material at www. liebertpub.com/overview/surgical-infections/53/) [361,362]. In a previously reviewed study [3], success rates in treating a confirmed candidal IAI appeared higher with amphotericin B than with fluconazole, but only very small numbers of patients were treated with either agent [363]. Among all patients with invasive candidal infections, a meta-analysis found no overall difference in treatment failure comparing amphotericin B 
formulations with echinocandins or azoles, but a better safety profile with echinocandins and less nephrotoxicity with fluconazole [364].

Fluconazole has been used extensively to treat patients with intra-abdominal candidal infections, but there are only limited new prospective data regarding this use. One RCT found higher success rates with anidulafungin compared with fluconazole in patients with invasive candidiasis, primarily from an abdominal source (Supplementary Table N; see online supplementary material at www.liebertpub.com/ overview/surgical-infections/53/) [365]. In the previous evidence review [3], one RCT showed that pre-emptive administration of fluconazole was more effective than placebo for preventing Candida peritonitis [366]; however, as indicated above, another RCT suggested fluconazole was less effective than amphotericin B in a very limited number of patients with abdominal candidiasis [363]. In patients with any type of candidal infection, the meta-analysis by GafterGvili et al. [364] identified a trend toward higher treatment failure rates with use of fluconazole, but this did not reach statistical significance.

There are few data available regarding management of intra-abdominal candidiasis with other azoles. Voriconazole has better in vitro activity than fluconazole against $C$. glabrata and $C$. krusei, although not all strains are susceptible [367369]. Voriconazole appeared to be equivalent to amphotericin B followed by fluconazole for the management of candidal infections in non-neutropenic patients, but the results in the very small number of patients with invasive candidiasis rather than candidemia were not reported separately [370].

Several RCTs have suggested that echinocandins are at least as effective as other agents for the treatment of patients with IAI from Candida spp.[361,362,365,371]. An uncontrolled trial of pre-emptive caspofungin suggested that this prevented an overt clinical infection from Candida in nearly all patients [372]. For the management of candidal infections in general, caspofungin and micafungin appear to be noninferior to amphotericin B formulations, and anidulafungin may be superior to fluconazole [364,373]. Adverse events appear to be lower with echinocandins than with polyenes or azoles. A cost-effectiveness analysis has suggested lower overall costs with use of an echinocandin compared with other agents, because of reduced adverse events, including persistent infection [374]. Guidelines by the IDSA recommend use of echinocandins over fluconazole in patients with moderately severe to severe illness from Candida [375].

Overall, the task force concluded that echinocandins can be recommended for the treatment of severely ill patients suspected or proven to have an IAI from Candida. Fluconazole can be used to treat less severely ill patients infected with $C$. albicans, but voriconazole or an echinocandin would be preferred for the treatment of patients infected with fluconazoleresistant species of Candida. Because less toxic alternatives are available, the task force does not recommend routine use of polyenes for treatment of patients with candidal IAI.

4.11a. We do not recommend routine use of amphotericin $B$ or its lipid formulations for empiric or pathogen-directed treatment of adults or children with intra-abdominal candidiasis (Grade 2-B).

$4.11 \mathrm{~b}$. We suggest use of fluconazole for pre-emptive management of IAI in non-critically ill adults and children who are at high risk for intra-abdominal candidiasis, and for pathogen-directed treatment of non-critically ill patients infected with susceptible strains of $\mathrm{C}$. albicans (Grade 2-B).

4.11c. We suggest use of voriconazole for empiric or pathogen-directed management of IAI in non-critically ill adults and children older than one month who are suspected or proven to be infected with strains of Candida that are not susceptible to fluconazole (Grade 2-B).

4.11d. We recommend an echinocandin (anidulafungin, caspofungin, or micafungin) for empiric or pathogendirected management of IAI in severely ill adults and children older than one month who are suspected or proven to be infected with Candida spp. (Grade 1-B).

\section{Oral antimicrobial agents}

Q 5.1. When can oral antimicrobial agents be used in the treatment of patients with IAI?

$\mathrm{Q}$ 5.2a. What is the role of oral amoxicillin-clavulanic acid in the treatment of patients with IAI?

Q 5.2b. What is the role of oral moxifloxacin in the treatment of patients with IAI?

$Q$ 5.2c. What is the role of oral ciprofloxacin plus metronidazole in the treatment of patients with IAI?

Q 5.2d. What other oral antimicrobial agents can potentially be used for the treatment of patients with IAI?

An accepted principle of antimicrobial stewardship is conversion from IV to oral anti-infective agents when the patient's condition permits [196]. This principle can be applied to patients with IAI as well and was recommended in previous guidelines $[2,4,5]$. As with IV antimicrobial agents, oral agents should have activity against the common aerobic gram-negative bacilli, gram-positive cocci, and anaerobic micro-organisms involved in these infections. Although the use of oral agents in patients with IAI is frequently not feasible immediately after a source control procedure because of gastrointestinal intolerance, it is often possible to switch the patient to oral agents later, once the patient has return of gastrointestinal function.

A number of RCTs have permitted use of oral antibiotics as a substitute for IV antibiotics. Only a few of these have directly tested the hypothesis that a switch to oral agents is equivalent to use of exclusive IV therapy for patients with IAI. In two studies, pediatric subjects were randomized to a regimen of IV antibiotics only or to a regimen of IV antibiotics in which a switch to oral amoxicillin-clavulanic acid was allowed after three or four days of IV therapy (Supplementary Table O; see online supplementary material at www. liebertpub.com/overview/surgical-infections/53/) [319,320]. An older study, reviewed previously [3], randomized one group of patients to an arm in which oral ciprofloxacin plus metronidazole was permitted as continuation therapy [376]. Outcomes were similar in patients allowed the oral switch and those who received IV antibiotics only.

Indirect evidence in support of a switch to oral antibiotic agents has come from additional RCTs in which patients were allowed to receive oral agents [215,217,229,245$247,249,251,288,289,301,377]$. It should be noted that many of these trials allowed for prolonged antimicrobial therapy, well beyond the previous recommendation of a maximum four to seven days of total antimicrobial therapy [2,4] or the current recommendation of a maximum of four days in adult 
patients or five days in pediatric patients (Sections 10 and 13). Thus, much of these data on use of oral antimicrobial therapy are irrelevant to current recommendations. Patients in these RCTs were frequently switched to oral agents different from the IV ones they had received, and in some cases to oral agents from an entirely different class of antibiotic. Thus, there does not appear to be an obligatory need to continue the same agent or agents when making a switch to oral therapy. Overall, the task force concluded that use of oral antimicrobial therapy is a valid option, but only if it shortens the course of IV therapy, and not if it is used to prolong the total duration of antimicrobial therapy beyond current recommendations.

Oral amoxicillin-clavulanic acid was directly evaluated as step-down therapy in two small RCTs of pediatric patients with perforated appendicitis [319,320], and allowed as stepdown therapy in a number of other trials [217,229,251, $288,301,320,377]$. No increase in treatment failure was observed in patients who were switched to this oral agent. There is a relatively high prevalence of $E$. coli resistant to amoxicillin-clavulanic acid throughout the world, however [219]. Thus, the task force would recommend some caution when using this agent for oral step-down therapy, and it should not be used if culture results reveal a resistant organism.

A switch from IV to oral moxifloxacin was permitted in several RCTs testing this agent $[214,228,250]$. There was no indication of any adverse events associated with a switch to oral moxifloxacin therapy. No trials directly compared patients permitted oral therapy with those assigned to receive IV therapy only, however. Use of oral moxifloxacin has not been studied in pediatric patients with IAI.

One relatively large RCT included in the previous evidence review [3] directly compared patients permitted a switch to oral ciprofloxacin plus metronidazole with patients who received IV therapy only with ciprofloxacin plus metronidazole or with imipenem-cilastatin. No significant differences in outcome were demonstrated between any of the study groups [376]. Additional trials also permitted a switch to oral ciprofloxacin plus metronidazole as an option [217,245247,249,301]. Clinical outcomes were not adversely affected by this switch to oral agents. Use of oral ciprofloxacin in pediatric patients appears to be relatively safe [303,304] and can be considered when other agents are not suitable.

There is relatively scant evidence with regard to the use of other oral agents for the treatment of patients with IAI. Oral first-, second-, and third-generation cephalosporins, including cephalexin, cefadroxil, cephradine, cefuroxime, cefaclor, cefprozil, cefdinir, and cefpodoxime, in combination with metronidazole, are potential options, as they have reasonable activity against non-ESBL-producing strains of E. coli [378385]. One small RCT found no adverse effect of a change from IV therapy to oral cephalexin, but this switch occurred fairly late during a prolonged course of antibiotic treatment [386]. Oral levofloxacin in combination with metronidazole is also a potential option, but there are no published RCTs describing use of either IV or oral levofloxacin in patients with IAI. Use of oral trimethoprim-sulfamethoxazole plus metronidazole as continuation therapy appeared to be successful in two observational studies in pediatric patients, but this switch also occurred fairly late in the antibiotic course [387,388].

5.1. We recommend use of selected oral agents with good bioavailability as a substitute for IV agents in the treatment of patients with IAI when the patient has return of adequate gastrointestinal function. Oral antibiotics should only be used to complete a short course of treatment and not to prolong antimicrobial use beyond current recommendations for duration of therapy (Grade 1-B).

5.2a. We suggest use of oral amoxicillin-clavulanic acid as an acceptable regimen to complete a short course of antimicrobial therapy for the treatment of IAI in adults and children older than one month with IAI (Grade 2-B).

$5.2 b$. We suggest use of oral moxifloxacin as an acceptable regimen to complete a short course of antimicrobial therapy for the treatment of IAI in adults (Grade 2-B). We do not recommend the use of oral moxifloxacin for the treatment of IAI in children, unless no other option is available (Grade 1-B).

$5.2 c$. We recommend use of oral ciprofloxacin plus metronidazole as an acceptable regimen to complete a short course of antimicrobial therapy for the treatment of IAI in adults (Grade 1-B). We suggest that oral ciprofloxacin plus metronidazole may be used to complete a short course of antimicrobial therapy for the treatment of IAI in children older than one month, if other options are not suitable (Grade 2-B).

$5.2 d$. We suggest that oral levofloxacin plus metronidazole, an oral first-, second-, or third-generation cephalosporin plus metronidazole, or oral trimethoprim-sulfamethoxazole plus metronidazole are potential regimens that could be used selectively to complete a short course of antibiotic therapy for the treatment of IAI in adults and children older than one month, if other oral agents are not suitable (Grade 2-C).

\section{Selection of empiric antimicrobial therapy for adult patients with CA-IAI}

\section{A. Lower-risk patients with CA-IAI}

Q 6.1. What are the general principles for selection of empiric antimicrobial therapy of CA-IAI in lower-risk patients?

Q 6.2a. What are the preferred agents for initial empiric antimicrobial therapy of CA-IAI in lower-risk patients?

Q 6.2b. Should patients with perforated appendicitis be treated with different empiric antimicrobial agents than other lower-risk patients with CA-IAI?

The established principle of antimicrobial therapy for patients with IAI has been to administer agents with activity against gram-negative Enterobacteriaceae, aerobic streptococci, and obligate enteric anaerobes $[1,2,4,5]$. As detailed in Section 4, a large number of antimicrobial agents or regimens meet these criteria, with efficacy information about many of them available from RCTs. In these clinical trials, lower-risk patients with CA-IAI have predominated, even in those evaluating broad-spectrum antimicrobial regimens [3,389]. Based on these trials, the breadth of antimicrobial coverage does not appear to have a major impact on clinical outcomes in lowerrisk patients with CA-IAI. Several RCTs published since 2000 have compared regimens with and without anti-pseudomonal activity [204,205,220,225,228-230,294-296] and with or without anti-enterococcal activity [204,205,225,228-230,252, 255,274].

By and large, these trials have not demonstrated significant differences in outcome when comparing narrow-spectrum and broad-spectrum regimens. A detailed meta-analysis concluded that antimicrobial regimens used for secondary peritonitis were all essentially equivalent [390]. A large database study 
did not identify any major differences in outcome comparing narrow-spectrum regimens with broad-spectrum regimens, as long as the regimen covered standard gram-negative Enterobacteriaceae and obligate anaerobes [192].

There are relatively little data available regarding use of antifungal agents in lower-risk patients with CA-IAI. Typically, RCTs have not permitted concomitant use of antifungal therapy. Treatment failures attributed to a lack of antifungal coverage, however, have rarely been evident in these trials. Moreover, microbiologic studies indicate that isolation of Candida spp. is quite uncommon in patients with CA-IAI [21,24,391-394].

Based on these data, the task force supports previous guidelines on avoiding use of broad-spectrum antimicrobial therapy, including antifungal therapy, in lower-risk patients with CA-IAI. Limiting the exposure of lower-risk patients with CA-IAI to broad-spectrum antimicrobial therapy would be consistent with the goals of antimicrobial stewardship programs [196,395,396]. In keeping with the goal of using narrow-spectrum agents for lower-risk patients with CA-IAI, the task force recommends use of agents that may not have much activity against Pseudomonas spp. or Enterococcus spp. These include ertapenem as monotherapy, and cefotaxime or ceftriaxone with metronidazole as combination therapy. Where available, cefoperazone-sulbactam is also an option. The use of cefuroxime plus metronidazole is reasonable but has been less well supported by contemporary data. Ticarcillin-clavulanic acid would be acceptable but will now only be an option if production resumes.

Use of fluoroquinolone-based regimens requires additional consideration. The finding that nearly $30 \%$ of $E$. coli strains isolated from patients with IAI in North America are resistant to fluoroquinolones is of concern [28], although the prevalence of resistant strains in lower-risk patients with CA-IAI may be lower than this. Recent clinical studies have described good results using moxifloxacin alone or ciprofloxacin plus metronidazole in patients with CA-IAI, despite being performed while there was increasing in vitro resistance of Enterobacteriaceae to fluoroquinolones [215,229,247,249$251,284]$. Thus, the task force recommends moxifloxacin or ciprofloxacin plus metronidazole for use in lower-risk patients with CA-IAI, but would prefer that such use be restricted primarily to patients with contraindications to the use of $\beta$-lactam antibiotics. A paucity of data exists supporting use of levofloxacin plus metronidazole for treatment of patients with IAI, and it has not been approved by the FDA for this indication. Because it may be the only fluoroquinolone available on some hospital formularies, however, the regimen of levofloxacin plus metronidazole is acceptable under those circumstances.

Table 9 outlines these recommendations for selection of empiric antimicrobial therapy in lower-risk patients with CAIAI. In regions of the world where there is a much higher prevalence of fluoroquinolone-resistant and ESBL-producing strains of Enterobacteriaceae in the community [28,235,397], these recommendations would likely need to be modified, as will be discussed further in Section $\mathrm{C}$ under recommendations 6.6.

Perforated appendicitis is usually the most common cause of IAI among participants in clinical trials [3]. Overall deaths are lower in these patients than in patients with IAI from other sources, even after adjusting for age and co-morbidities [59,75,187,398]. Post-operative infections, however, including intra-abdominal abscesses, occur in a substantial number
TABle 9. Recommended Empiric Antimicrobial REgimens FOR PATIENTS WITH COMMUNITYACQUIRED INTRA-ABDOMINAL INFECTION

\begin{tabular}{|c|c|}
\hline Lower-risk patients ${ }^{\mathrm{a}, \mathrm{b}}$ & Higher-risk patients \\
\hline \multicolumn{2}{|l|}{ Single agents } \\
\hline Ertapenem & Piperacillin-tazobactam \\
\hline \multirow{3}{*}{ Moxifloxacin ${ }^{c}$} & Doripenem $^{\mathrm{f}}$ \\
\hline & Imipenem-cilastatin \\
\hline & Meropenem ${ }^{\mathrm{f}}$ \\
\hline \multicolumn{2}{|l|}{ Combination regimens } \\
\hline $\begin{array}{l}\text { Cefotaxime or ceftriaxone } \\
\text { plus metronidazole }\end{array}$ & $\begin{array}{l}\text { Cefepime plus } \\
\text { metronidazole }\end{array}$ \\
\hline $\begin{array}{l}\text { Ciprofloxacin plus } \\
\text { metronidazole } e^{\mathrm{c}, \mathrm{e}}\end{array}$ & $\begin{array}{l}\text { Aztreonam plus } \\
\text { metronidazole plus } \\
\text { vancomycin }\end{array}$ \\
\hline
\end{tabular}

${ }^{a}$ Ticarcillin-clavulanate is no longer available in the United States.

${ }^{\mathrm{b}}$ Cefoperazone-sulbactam is also an option, where available.

${ }^{\mathrm{c}}$ Use of fluoroquinolones is suggested primarily for patients with significant reactions to $\beta$-lactam antibiotic agents.

${ }^{\mathrm{d}}$ Cefuroxime plus metronidazole is also an option, but is less well supported by contemporary data.

${ }^{\mathrm{E}}$ If levofloxacin is the only fluoroquinolone available on a formulary, it may be substituted for ciprofloxacin. There is little evidence with regard to its efficacy, and it is not approved by the Food and Drug Administration for treatment of patients with complicated intra-abdominal infection.

${ }^{\mathrm{f}}$ Use of an agent such as ampicillin or vancomycin effective against Enterococcus spp. can be considered in patients with severe sepsis-septic shock and other higher-risk patients who receive doripenem or meropenem, and should be added to a cephalosporinbased regimen.

${ }^{\mathrm{g}}$ Ceftazidime plus metronidazole is also an option, but is less well supported by contemporary data.

${ }^{\mathrm{h}}$ Aztreonam plus metronidazole plus vancomycin is an option for patients with significant reactions to $\beta$-lactam antibiotic agents, but is less well supported by contemporary data.

of patients with appendiceal perforations [187,399-401]. These treatment failures are associated with longer hospitalizations and increased use of resources [192]. Thus, the task force believes that the same antimicrobial regimens outlined for use in lower-risk patients with CA-IAI apply to lower-risk patients with perforated appendicitis, and that potentially lower cost but inferior regimens should not be substituted.

6.1. We recommend treatment of CA-IAI in lower-risk patients with narrower-spectrum antimicrobial agents having activity against the usual gram-negative Enterobacteriaceae, aerobic streptococci, and obligate anaerobic microorganisms associated with these infections (Grade 1-A). We recommend against the use of broaderspectrum or additional agents specifically to provide antipseudomonal or anti-enterococcal coverage (Grade 1-A). We suggest that antifungal coverage is unnecessary for management of CA-IAI in lower-risk patients (Grade 2-B).

$6.2 a$. We recommend cefotaxime or ceftriaxone plus metronidazole or ertapenem as the preferred antimicrobial agents for the management of CA-IAI in lower-risk patients (Grade 1-A). We recommend ciprofloxacin plus metronidazole or moxifloxacin monotherapy for the management of CA-IAI in lower-risk patients who have serious $\beta$-lactam allergies (Grade 1-A), and suggest levofloxacin plus metronidazole as an alternative if no other fluoroquinolone is available (Grade 2-C). We suggest use of cefuroxime plus metronidazole (Grade 
2-B), or cefoperazone-sulbactam, where available (Grade 2$B)$, as alternative empiric antimicrobial regimens for the management of CA-IAI in lower-risk patients.

$6.2 b$. We recommend treatment of patients with perforated appendicitis with the same agents or regimens recommended for other lower-risk patients with CA-IAI, unless they meet criteria identifying them as higher-risk patients or at risk for having resistant pathogens (Grade 1-A).

\section{B. Higher risk patients with CA-IAI}

Q 6.3. What are the general principles for selection of empiric antimicrobial therapy of CA-IAI in higher-risk patients?

Q 6.4a. What are the preferred agents for initial empiric antimicrobial therapy of CA-IAI in higher-risk patients? Q 6.4b. Should empiric anti-enterococcal therapy be used to treat CA-IAI in higher-risk patients?

Q 6.4c. Should empiric antifungal therapy be used to treat CA-IAI in higher-risk patients?

Inadequate antimicrobial therapy has been associated with higher deaths in patients with sepsis and septic shock from any source [193-195]. A number of studies have also demonstrated an association of inadequate antimicrobial therapy with worse outcomes in patients with IAI [43,54,58,71,187,194], although this finding has not been replicated by all investigators $[18,402,403]$. The infecting flora of higher-risk patients with CA-IAI are generally similar to those of lower-risk patients. Some patients with CA-IAI, however, are infected with pathogens such as Enterobacter spp., P. aeruginosa and Enterococcus spp., which are resistant to the narrower-spectrum agents recommended for use in lower-risk patients [22$24,26,27]$. Higher-risk patients infected with these pathogens might therefore receive inadequate antimicrobial therapy if narrower-spectrum agents were used.

A limited number of observational studies have suggested that the failure to treat seriously ill patients infected with micro-organisms such as Enterococcus spp. and $P$. aeruginosa does result in higher deaths [24,111,330,404]. The task force therefore suggests that higher-risk patients with CAIAI, particularly those with sepsis or septic shock, receive broader-spectrum empiric antimicrobial therapy to minimize the risk of inadequate initial therapy. The agents that have been included in this category are piperacillin-tazobactam, the broad-spectrum carbapenems, imipenem-cilastatin, meropenem, and doripenem, and cefepime plus metronidazole. The use of ceftazidime plus metronidazole is an option, although less well supported by contemporary data. Similarly, the use of aztreonam plus metronidazole plus vancomycin can be considered, particularly for patients with severe $\beta$ lactam reactions, although it too has not been evaluated in recent publications (Table 9).

Previous guidelines did not support the use of combination gram-negative therapy when treating higher-risk patients with CA-IAI. MDR gram-negative pathogens are uncommon in higher-risk patients with CA-IAI [24]. Two RCTs published since 2000 did not find the combination of an aminoglycoside with a cephalosporin to be any more efficacious than use of a cephalosporin-based regimen alone, although different agents were used in each arm of these trials [203,254]. Another large RCT did not identify any improvement in outcome with addition of moxifloxacin to meropenem for treat- ment of patients with sepsis; this study included a large number of patients with IAI, but did not report the results of those patients separately [405]. A recent meta-analysis did not find any benefit of combination regimens on outcomes in patients with bacteremia because of $P$. aeruginosa [406]. Two randomized trials included in the previous evidence review [3] did not demonstrate any improvement in outcome with addition of an aminoglycoside to a $\beta$-lactam antibiotic agent in severely ill patients with IAI $[22,407]$. The task force, therefore, does not believe that combination gram-negative therapy is necessary for empiric treatment of higher-risk patients with CA-IAI.

Although there is strong evidence that anti-enterococcal therapy is unnecessary in lower-risk patients with CA-IAI, it is less clear if this applies to higher-risk patients with CA-IAI. Most of the evidence is derived from observational studies. The frequency with which Enterococcus spp. is isolated appears to be increased in higher-risk as compared with lowerrisk patients with CA-IAI [27,89,408]. Isolation of Enterococcus spp. has also been associated with worse clinical outcomes, including death, in some studies of higher-risk patients with CA-IAI [24,27,111]. Thus, the task force concluded that there is rationale for providing empiric antienterococcal coverage in seriously ill patients with CA-IAI.

Although both E. faecalis and E. faecium have been isolated from peritoneal cultures of patients with CA-IAI, E. faecalis predominates [24,26]. Thus, coverage of $E$. faecalis is likely to be the primary concern when potentially using an anti-enterococcal agent to treat higher-risk patients with CA-IAI. Ampicillin and piperacillin generally have good activity against $E$. faecalis in vitro [89,324,409]. Among the broad-spectrum carbapenems, imipenem-cilastatin also has good in vitro activity, but doripenem and particularly meropenem have somewhat less activity [329,332,409-411]. Based on these data, the task force judged piperacillintazobactam or imipenem-cilastatin, and the regimen of aztreonam, metronidazole, and vancomycin as adequate for empiric treatment of Enterococcus spp. in higher-risk patients with CA-IAI. With use of other broad-spectrum carbapenems, addition of ampicillin or vancomycin could be considered. Supplemental ampicillin or vancomycin for coverage of E. faecalis should be added routinely to combinations of cefepime or ceftazidime with metronidazole, because these regimens lack anti-enterococcal coverage. Routine therapy directed against E. faecium was not considered necessary for higher-risk patients with CA-IAI, but if there were a high suspicion that this organism was a component of the infecting flora, supplemental vancomycin should be used.

It is also uncertain whether the recommendation against use of empiric antifungal therapy in lower-risk patients with CA-IAI should apply to higher-risk patients with CA-IAI. Observational studies have provided somewhat contradictory findings as to whether or not isolation of Candida is associated with increased deaths in critically ill patients with CA-IAI [56,412]. One RCT, which included critically ill patients with both CA-IAI and HA-IAI, did not find empiric use of fluconazole associated with any improvement in outcome [413]. Retrospective studies have also failed to document a benefit to routine use of empiric antifungal therapy in patients with CA-IAI $[27,414]$. Thus, in agreement with other authorities $[56,415]$, the task force believes that routine antifungal therapy is unnecessary for most higher-risk patients with CA-IAI. 
Critically ill patients with CA-IAI from upper gastrointestinal perforations, however, have a particularly high incidence of Candida peritonitis [110,416,417], and the overall deaths in these patients is quite high $[412,418]$. A retrospective study of patients with gastroduodenal perforations suggested that delayed antifungal therapy contributed to increased deaths [416]. Based on these limited data, the task force suggested that empiric antifungal therapy be considered for critically ill patients with CA-IAI because of upper gastrointestinal perforations.

6.3. We suggest treatment of CA-IAI in higher-risk patients with broader-spectrum empiric antimicrobial agents to ensure coverage of less common gram-negative pathogens potentially involved in these infections (Grade 2-C).

6.4a. We suggest piperacillin-tazobactam, imipenemcilastatin, meropenem, doripenem, or cefepime plus metronidazole as the preferred antimicrobial agents for empiric treatment of CA-IAI in higher-risk patients (Grade 2-A). We suggest ceftazidime plus metronidazole as an alternative regimen for these patients (Grade 2-B). We suggest aztreonam plus metronidazole plus vancomycin for empiric treatment of patients with a severe reaction to $\beta$-lactam agents (Grade 2-B). We do not recommend addition of an adjunctive aminoglycoside or fluoroquinolone to a $\beta$-lactam agent for empiric management of CA-IAI in higher-risk patients (Grade 1-B).

6.4b. We suggest addition of ampicillin or vancomycin for empiric anti-enterococcal management of CA-IAI in higherrisk patients, if the patient is not being treated with piperacillin-tazobactam or imipenem-cilastatin (Grade 2-B).

$6.4 c$. We do not recommend routine use of empiric antifungal therapy for management of CA-IAI in higher-risk patients (Grade 1-B). We suggest empiric use of antifungal therapy for management of CA-IAI in critically ill patients with an upper gastrointestinal source (Grade 2-B).

C. Other considerations for empiric antimicrobial therapy of patients with CA-IAI

Q 6.5. What agents can be used for initial empiric antimicrobial therapy of CA-IAI in patients with major reactions to $\beta$-lactam antibiotics?

Q 6.6. What agents can be used for initial empiric antimicrobial therapy of CA-IAI in patients who reside in geographic areas with a high prevalence of ESBLproducing Enterobacteriaceae in the community?

Q 6.7. What agents can be used for initial empiric antimicrobial therapy of CA-IAI in patients who reside in geographic areas where there are major resource limitations precluding use of recommended antimicrobial agents?

Antibiotic selection may be problematic in patients with hypersensitivity reactions to $\beta$-lactam antibiotic agents. Although $5 \%-10 \%$ of patients report a history of a reaction to penicillin, anaphylactic reactions are uncommon. Moreover, the majority of patients reporting a penicillin allergy do not in actuality have one $[283,419,420]$. Cross-reactivity between various $\beta$-lactam agents is relatively uncommon, and monobactams and carbapenems are generally safe to use in almost all patients with reactions to penicillins or cephalosporins, even those with serious reactions [283,421,422]. Nonetheless, frequently there is a reluctance on the part of clinicians to prescribe a $\beta$-lactam antibiotic agent to any patient with even a very vague history of a penicillin or other $\beta$-lactam allergy.

Based on the review of this evidence and expert opinion, the task force has identified a fluoroquinolone-based regimen as a reasonable option for initial empiric treatment of lowerrisk patients with CA-IAI who have a serious reaction to $\beta$ lactam antibiotic agents. If the patient cannot receive a fluoroquinolone antibiotic agent, a $\beta$-lactam antibiotic agent with a low potential for cross-reaction with the agent producing the allergy could be considered. Non- $\beta$-lactam alternatives such as an aminoglycoside plus metronidazole or tigecycline are also options, but the potential for decreased efficacy of these alternative agents is a concern.

For higher-risk patients with CA-IAI, alternatives to $\beta$ lactam antibiotic agents may be quite limited because of efficacy concerns. The task force has selected aztreonam plus metronidazole plus vancomycin as a reasonable alternative for patients with severe $\beta$-lactam reactions, because aztreonam has almost no cross-reactivity with other $\beta$-lactam antibiotic agents. A $\beta$-lactam from a different class could also be considered, given the relatively low cross-reactivity between $\beta$-lactams. Use of a fluoroquinolone-based regimen is a less-attractive option, because of the relatively widespread prevalence of fluoroquinolone-resistant Enterobacteriaceae. The potential for decreased efficacy of aminoglycoside-based regimens and tigecycline would also argue against their use as monotherapy for management of CA-IAI in higher-risk patients with severe $\beta$-lactam reactions.

The increasing prevalence of ESBL-producing Enterobacteriaceae in parts of Latin America, Asia, and Southern Europe makes selection of empiric antimicrobial therapy for patients with CA-IAI in those regions more problematic. Common ESBL-producing strains should be considered resistant to aztreonam and most cephalosporins other than cephamycins regardless of in vitro testing. Moreover, ESBL-producing Enterobacteriaceae are frequently resistant to cephamycins and to fluoroquinolones through other mechanisms [29,235,397,423,424-428]. Susceptibilities of ESBL-producing strains of Enterobacteriaceae to tigecycline are generally high, although susceptibilities to piperacillin-tazobactam and amikacin are somewhat more variable [28,235,241,429,430]. Carbapenems have reliable activity against most ESBL-producing Enterobacteriaceae except for those producing carbapenemases [28,29,235,431].

Based on these data, the task force considers ertapenem to be the best option for empiric treatment of lower-risk patients with CA-IAI in regions where there is a high likelihood that ESBL-producing Enterobacteriaceae are components of the infection. Tigecycline or an aminoglycoside-based regimen is an alternative, although less preferable because of efficacy concerns. Where available, cefoperazone-sulbactam is another potential option, especially since one RCT demonstrated its efficacy in managing IAI in a region where there is a high prevalence of ESBL-producing Enterobacteriaceae [203]. Based on in vitro susceptibility patterns, most cephalosporins, including cephamycins, aztreonam, and fluoroquinolones, would not be good options [423,424,432]. Nonetheless, recent RCTs have not generally demonstrated higher clinical failure rates in patients with IAI who were treated with agents that have poorer activity against ESBLproducing micro-organisms [203,205,214,220,246,252,253, 255,284,293]. 
Based on the principle of ensuring adequate initial empiric antimicrobial therapy, the task force would select a broadspectrum carbapenem as the first choice in treating higher-risk patients with CA-IAI who reside in regions with a high prevalence of ESBL-producing Enterobacteriaceae in the community $[423,424,432]$. Another option could be piperacillin-tazobactam [309], although many authorities advise caution with its use $[423,424,432,433]$. The potential for decreased efficacy of aminoglycosides and tigecycline for treating higher-risk patients with CA-IAI argues against their use as empiric therapy. Based on in vitro susceptibility profiles and limited clinical data, ceftolozane-tazobactam and ceftazidimeavibactam could be options for empiric therapy [263, 264,266,274-276], but these agents are not yet available in many parts of the world where there is a high prevalence of ESBL-producing Enterobacteriaceae. Because heavy use of carbapenems has been associated with selection of carbapenemase-producing gram-negative bacilli [298,432, 434,435], de-escalation from empiric carbapenem therapy should be undertaken, whenever possible.

Limitations on the availability of antimicrobial resources in many parts of the world may preclude use of recommended antibiotic regimens to treat patients with IAI. Although the task force recommendations are based primarily on evidence of efficacy, the absolute risk of a worse outcome with use of a non-recommended regimen compared with a recommended regimen may be relatively small, as illustrated in metaanalyses comparing use of aminoglycoside-based regimens to other regimens [207,208]. Inadequate source control is much more likely than selection of a specific empiric antimicrobial regimen to lead to an adverse outcome in patients with IAI $[51,61,71,436]$. Thus, ensuring access to appropriate source control may have a larger impact than providing ideal, but expensive antimicrobial agents in areas of resource limitations.

Non-recommended agents or regimens such as ampicillinsulbactam, amoxicillin-clavulanate, cefoxitin, cefotetan, or cefazolin or aminoglycosides in combination with metronidazole or clindamycin have an appropriate spectrum of activity for treating patients with IAI and could be used if available. Recommended oral agents would also be lower-cost alternatives to more expensive IV agents. Nonetheless, the potential for decreased efficacy or increased side effects related to use of non-recommended agents could negate any of the cost benefits from using less expensive pharmaceuticals.

6.5. We suggest use of a fluoroquinolone-based regimen for initial empiric antimicrobial therapy of CA-IAI in lowerrisk patients who have had major reactions to $\beta$-lactam antibiotic agents (Grade 2-B). We suggest use of an aztreonambased regimen for initial empiric therapy of CA-IAI in higher-risk patients who have had major reactions to $\beta$ lactam antibiotic agents (Grade 2-B). We suggest that a nonpenicillin $\beta$-lactam may be used for empiric antimicrobial therapy of CA-IAI in patients for whom a severe penicillin allergy has not been documented and for whom the riskbenefit ratio is believed acceptable (Grade 2-B).

6.6. We recommend against empiric use of most cephalosporin-, aztreonam-, or fluoroquinolone-based regimens for empiric antimicrobial therapy of CA-IAI in patients who reside in geographic areas where there is a high prevalence of ESBL-producing Enterobacteriaceae in the community
(Grade 1-B). We recommend use of ertapenem for empiric antimicrobial therapy of CA-IAI in lower-risk patients or a broad-spectrum carbapenem (doripenem, imipenemcilastatin, or meropenem) for CA-IAI in higher-risk patients who reside in such areas (Grade 1-B).

6.7. We suggest that locally available IV or oral agents having activity against common intra-abdominal pathogens be used for empiric antimicrobial therapy of CA-IAI in patients who reside in geographic areas where there are major resource limitations (Grade 2-C).

\section{Selection of empiric antimicrobial therapy for adult patients with $H A-I A I$}

\section{A. General approach}

Q 7.1a. How should patients with HA-IAI be assessed with respect to their risk of infection with resistant or opportunistic pathogens?

Q 7.1b. What agents can be used for initial empiric antimicrobial therapy of HA-IAI in patients who are identified as being at risk of resistant pathogens?

The criteria for identifying patients with HA-IAI were discussed in Section 1 and are listed in Table 7. Epidemiologic studies have identified a number of risk factors identifying patients with HA-IAI at risk for harboring resistant or opportunistic pathogens $[15,17,19,22-24,26,32,33,62,77,79$, 85-90,110,340,417,418]. The task force believes that Enterococcus spp., MRSA, resistant gram-negative bacilli, and Candida spp. are the resistant or opportunistic pathogens of primary concern for purposes of prescribing antimicrobial therapy. It is therefore suggested that a patient be assessed individually as to his or her risk of infection with each of these distinct micro-organisms. Knowledge of the local microbial ecology as well as the patient's exposure to previous antimicrobial therapy will also influence antimicrobial selection.

Because of the wide variety of infecting micro-organisms in patients with HA-IAI and the frequency with which these pathogens are resistant to antimicrobial agents, these patients are at risk for a worse outcome because of inadequate empiric antimicrobial therapy $[43,54,58,187,193-195]$. At a minimum, the task force believes that patients with HA-IAI should receive the empiric antimicrobial regimens described for use in higher-risk patients with CA-IAI. The recommended agents include piperacillin-tazobactam, cefepime plus metronidazole, or a broad-spectrum carbapenem; ceftazidime plus metronidazole or aztreonam plus metronidazole plus vancomycin are potential alternatives (Table 10). The potential pathogenic role of enterococci in postoperative infections probably warrants use of an empiric antienterococcal agent in most of these patients as well. Addition of other agents to this basic regimen should be predicated on the individualized assessment of the patient's risk for resistant gram-positive, resistant gram-negative, or fungal pathogens $[17,18,33,62,197,241,437,438]$.

7.1a. We suggest that all patients with HA-IAI be assessed with respect to their separate risks of infection from Enterococcus spp., MRSA, resistant gram-negative bacilli, and Candida spp. (Grade 2-B).

7.1b. We suggest that broader-spectrum agents or regimens described for use in higher-risk patients with CA-IAI be used for the initial empiric antimicrobial therapy of patients 
Table 10. Summary of Empiric Antimicrobial Therapy for Patients with Healthcare- Or Hospital-Acquired Intra-Abdominal Infection

General approach

Piperacillin-tazobactam, doripenem, imipenem-cilastatin, meropenem, or cefepime plus metronidazole, with ceftazidime plus metronidazole and aztreonam plus metronidazole plus vancomycin as potential alternatives

\section{Supplemental agents}

Potential pathogen

Enterococcus faecalis

Enterococcus faecium

Vancomycin-resistant Enterococcus spp.

MRSA

ESBL-producing or AmpC- $\beta$-lactamaseproducing Enterobacteriaceae

KPC-producing Enterobacteriaceae

MDR strains of Pseudomonas aeruginosa

MDR strains of Acinetobacter baumannii

Candida albicans

Non-C. albicans spp.
Recommendations

Addition of ampicillin or vancomycin if not using piperacillintazobactam or imipenem-cilastatin

Vancomycin or teicoplanin

Daptomycin or linezolid

Vancomycin, teicoplanin, daptomycin, or linezolid

Use of a broad-spectrum carbapenem

Combination therapy with a broad-spectrum carbapenem plus an aminoglycoside, polymyxin, or tigecycline; or ceftazidime-avibactam

Combination therapy with an aminoglycoside plus colistin, or ceftolozane-tazobactam or ceftazidime-avibactam

Combination therapy with a broad-spectrum carbapenem plus an aminoglycoside, polymyxin, or tigecycline

An echinocandin (anidulafungin, caspofungin, micafungin)

for critically ill patients, fluconazole for less critically ill patients An echinocandin

MRSA = methicillin-resistant Staphylococcus aureus $; \mathrm{ESBL}=$ extended-spectrum beta-lactamase $; \mathrm{KPC}=$ Klebsiella pneumoniae carbapenemase.

with HA-IAI. Other empiric agents should be added to the regimen according to the assessment of the patient's risk for an infection from Enterococcus spp., MRSA, resistant gramnegative bacilli, and Candida spp. (Grade 2-B).

\section{B. Anti-enterococcal therapy}

Q 7.2a. How should patients with HA-IAI be assessed with respect to their risk of infection because of Enterococcus spp.?

Q 7.2b. Which agents can be used for initial empiric antimicrobial therapy of HA-IAI in patients considered at risk for infection with Enterococcus spp.?

Enterococci are common opportunistic micro-organisms isolated from patients with HA-IAI, although their pathogenic role in IAI is still a matter of some debate. Isolation of enterococci has been associated with a higher risk for an adverse outcome in many, but not all studies [24,27,49, $58,86,88,89,119,403,408]$. Patients in whom HA-IAI develops after an abdominal operation appear to be at particular risk for infection from Enterococcus spp. [26,86,88,89,439], as are patients who have received previous broad-spectrum antibiotic therapy [111,119]. Other risk factors for isolation of enterococci in patients with IAI include co-morbid conditions such as heart disease, chronic obstructive pulmonary disease, and malignancy; higher APACHE II, Simplified Acute Physiology Score (SAPS) II, or Sequential Organ Failure Assessment (SOFA) scores; previous hepatobiliary instrumentation; a history of solid organ transplantation, particularly liver transplantation; and immunosuppressive therapy [88,89,111,119,439].

Both E. faecalis and E. faecium are encountered relatively frequently in patients with HA-IAI, with the latter microorganism accounting for a substantial proportion of these enterococcal isolates [24,26,106,119,408,439,440]. Moreover, VRE is being identified increasingly in patients with enterococcal infections, including HA-IAI, in several areas of the world [26,115,441,442]. Recent reports suggest that VRE accounts for more than one-third of enterococcal infections in the United States, with most of these because of vancomycinresistant E. faecium [102,106]. The risk of an infection from VRE is much higher in patients already colonized with VRE $[93,96,102,103,106]$. Risk factors for colonization and infection with VRE also include a prolonged hospital length of stay, particularly in the intensive care unit, previous surgical procedures, a high severity of illness or extensive comorbidities, and substantial previous exposure to broadspectrum antibiotic therapy [102,103,106,115]. Solid organ transplant patients, especially liver transplant patients, are a particularly high-risk group of patients for VRE colonization and infection [103,115,443].

There are conflicting data as to whether or not timely antienterococcal therapy improves outcomes in patients infected with enterococci $[49,86,89,111,119,408,411]$. Methodologically robust trials of anti-enterococcal therapy in higher-risk patients are lacking. Because of the potential for poor outcomes with a failure to provide adequate antimicrobial therapy, however, the task force supports use of antienterococcal therapy in patients with HA-IAI identified as being at risk for infection with Enterococcus spp. This therapy should be selected on the basis of the likely species and strains that will be encountered.

Because of the higher risk that E. faecium is a pathogen in patients with HA-IAI, empiric anti-enterococcal therapy should be directed against both E. faecalis and E. faecium. Glycopeptides generally have good activity against non-VRE strains of E. faecalis and E. faecium, whereas $\beta$-lactam antibiotic agents typically lack activity against E. faecium $[324,329,331,332,409,444]$. Therefore, the task force suggests use of vancomycin or teicoplanin for patients with HA-IAI at risk for infection with Enterococcus spp. For patients with HAIAI considered at high risk for infection from VRE, however, the task force suggests use of empiric linezolid or daptomycin. 
These agents generally have good activity against VRE, with linezolid having been approved by the FDA for management of such infections [93,106,115,333,335,445]. Tigecycline also has good in vitro activity against VRE, but there are only limited clinical data regarding use of this agent for patients with HA-IAI because of VRE $[443,446,447]$. These suggestions for anti-enterococcal therapy in patients with HA-IAI are summarized in Table 10. With empiric use of any type of antienterococcal agent, treatment should be discontinued or deescalated if a resistant enterococcal strain is not identified in definitive cultures.

7.2a. We suggest that patients with HA-IAI who have a post-operative infection, have had substantial recent exposure to broad-spectrum antimicrobial therapy, who manifest signs of severe sepsis or septic shock, or who are known to be colonized with VRE be considered at risk for infection from Enterococcus spp. (Grade 2-B).

7.2b. We suggest vancomycin or teicoplanin for empiric antimicrobial therapy of HA-IAI in patients considered at risk for an infection from Enterococcus spp. We suggest use of linezolid or daptomycin for management of HA-IAI in patients known to be colonized with VRE or considered at high risk for infection from this organism (Grade 2-B).

\section{Anti-staphylococcal therapy}

Q 7.3a. How should patients with HA-IAI be assessed with respect to their risk of infection with MRSA?

Q 7.3b. Which agents can be used for initial empiric antimicrobial therapy of HA-IAI in patients considered to be at risk for infection with MRSA?

Staphylococci are not common pathogens in IAI. Coagulase-negative staphylococci are most often described in the context of tertiary peritonitis or recurrent IAI, although their pathogenic role is uncertain $[32,85,90,448]$. $S$. aureus is rarely isolated from peritoneal cultures of patients with CAIAI [23,24,27,62], but is found somewhat more frequently in cultures from patients with HA-IAI; many of these isolates are resistant to methicillin [23,24,27,62,340,341,342].

Patients colonized with MRSA are at risk for invasive MRSA infections [94,97,98,100], including post-operative HA-IAI [340]. Risk factors for MRSA colonization, which overlap with those for HAI in general, include advanced age, female gender, major medical co-morbidities, previous exposure to healthcareassociated pathogens, residence in a long-term care facility, recent hospitalization, recent surgery, and a history of recent exposure to antibiotic agents [100,449-451]. The task force suggests that patients known to be colonized with MRSA and those who have several risk factors for MRSA colonization be considered at risk for HA-IAI because of MRSA.

There are no published studies specifically evaluating antimicrobial therapy for IAI related to MRSA. Glycopeptides are the agents prescribed most commonly for patients with serious infections from MRSA [334,343-345,358]. Thus, vancomycin or teicoplanin (where available) could be used for empiric therapy of HA-IAI if MRSA is a suspected pathogen. The higher dosages of vancomycin advocated for managing infections from MRSA [344,452] may be leading to higher rates of nephrotoxicity with this agent, however [350-352]. In addition, the overall efficacy of vancomycin for serious MRSA infections has been called into question [345-348].
Thus, use of different agents for first-line therapy of MRSA infections should also be considered [353-355] Both linezolid and daptomycin appear to have efficacy at least equivalent to vancomycin for managing serious infections from this organism and have been used successfully in patients with IAI [344,348,354-359]. Based on these data, the task force believes that vancomycin, teicoplanin (where available), linezolid, and daptomycin are options for the empiric treatment of patients with HA-IAI when MRSA is a suspected pathogen (Table 10). The task force did not encounter sufficient published data regarding management of serious MRSA infections with other agents active against this organism, including ceftaroline, tigecycline, telavancin, dalbavancin, and oritavancin, to make any recommendation regarding use of these agents.

7.3a. We suggest that patients with HA-IAI known to be colonized with MRSA and those with multiple healthcareassociated risk factors for MRSA colonization, including advanced age, co-morbid medical conditions, previous hospitalization or surgery, and significant recent exposure to antibiotic agents, be considered at risk for infection from MRSA (Grade 2-B).

7.3b. We suggest vancomycin or teicoplanin, where available, or linezolid or daptomycin as alternatives, for empiric antimicrobial therapy of HA-IAI in patients known to be colonized with MRSA or considered at high risk for infection from this organism (Grade 2-B).

D. Antibacterial therapy for resistant gram-negative organisms

Q 7.4a. Which patients with HA-IAI should be considered at risk for infection from resistant gram-negative pathogens?

Q 7.4b. What other factors should be considered in selecting empiric antimicrobial therapy for patients with HAIAI from suspected resistant gram-negative pathogens?

Q 7.4c. What agents can be used for empiric antimicrobial therapy of HA-IAI in patients considered at risk for resistant Enterobacteriaceae?

Q 7.4d. What agents can be used for empiric antimicrobial therapy of HA-IAI in patients considered at risk for MDR, or XDR, or pan-drug-resistant (PDR) strains of P. aeruginosa or Acinetobacter spp.?

Compared with patients with CA-IAI, those with HA-IAI are infected with a much wider variety of gram-negative pathogens, many of which are resistant to various antibiotic agents [437,453]. Although E. coli is still the most common gram-negative pathogen identified in HA-IAI, other Enterobacteriaceae, such as Enterobacter spp., and non-lactosefermenting gram-negative pathogens, such as $P$. aeruginosa and $A$. baumannii, are encountered with some frequency [22$28,33,62,454]$. These pathogens develop resistance to antibiotic agents through a variety of mechanisms, including production of an ESBL or an aminoglycoside-modifying enzyme, elaboration of efflux pumps, loss of porins, or target site modifications. Simultaneous resistance to several classes of antibiotic agents is relatively common with many of these gram-negative bacteria [239,256,285,317,432,455-458].

The risk factors for HA-IAI because of resistant gramnegative pathogens mirror those for HA-IAI in general. Hospitalization, recent medical interventions, and pre-existing 
medical co-morbidities are all associated with infection from these resistant microorganisms [17-19,62,104,439]. Numerous studies have also closely linked previous broad-spectrum antimicrobial therapy with colonization or infection because of resistant gram-negative organisms [17-19,99,112-114,116118,120-123]. Unfortunately, these risk factors are quite nonspecific and are probably only useful in the extreme for identifying patients with HA-IAI likely to be infected with one of these pathogens. Patients known to be previously colonized or infected with a resistant gram-negative organism, however, can be suspected of having HA-IAI because of that agent. Routine surveillance for resistant gram-negative organisms, however, is rarely performed [91,92,95,99,101,104,105], so this information will only be available for a minority of patients with HA-IAI.

The local microbial ecology may be the most important aspect to consider in evaluating the risk that a patient with HAIAI is infected with a resistant gram-negative micro-organism. Strains of these resistant bacteria are endemic in certain geographic locales $[239,432,434,435,437,453,455,459,460]$; this phenomenon may extend to the individual institution or even to a specific unit within that institution [461-463]. Thus, updated hospital- or unit-specific antibiograms, where available, may help determine when a patient with HA-IAI is at risk for infection with a specific resistant gram-negative pathogen [194,462,463]. Overall, the task force believes that risk factors such as prolonged hospitalization, multiple medical interventions, previous broad-spectrum antimicrobial therapy, or a history of colonization or infection with resistant microorganisms should be considered along with local epidemiologic information in identifying patients with HA-IAI at risk for infection from resistant gram-negative pathogens.

Selection of empiric antimicrobial therapy for HA-IAI will be based not only on the general risk that the patient is infected with a resistant gram-negative micro-organism, but also on the specific risk for a given pathogen or pathogens. The goal is to select an empiric regimen likely to be active against the resistant pathogens the patient may carry, so that the risk of inadequate antimicrobial therapy is minimized. The task force has concluded that the agents with broadspectrum activity against gram-negative micro-organisms that were recommended for treatment of higher-risk patients (Table 10) form the basis for empiric therapy of patients with HA-IAI. Alternative or additional agents for gram-negative coverage may be warranted, however, if the patient is at risk for an MDR- or XDR-pathogen.

Patients with HA-IAI are at risk for infections with ESBLproducing Enterobacteriaceae, although the risk of this is highest in certain geographic regions of the world, such as Asia and Latin America [28,29,31]. Carbapenems are generally considered the empiric agents of choice for treating patients with the most common ESBL-producing Enterobacteriaceae (Table 10) [309,464-466], To avoid excessive carbapenem use, however, de-escalation to other agents, such as piperacillin-tazobactam, cefepime, an aminoglycoside, a cephamycin, a fluoroquinolone, or tigecycline, can be considered once susceptibilities are known. Unfortunately, with the exception of tigecycline, ESBL-producing Enterobacteriaceae are frequently resistant to these other agents [206,257,423,424,432,467]. Both ceftolozane-tazobactam and ceftazidime-avibactam have shown efficacy in treating patients with IAI caused by ESBL-producing Enterobacteriaceae, par- ticularly $E$. coli, but clinical experience with these agents is still limited [263,264,274,275].

Patients with HA-IAI who have had extensive exposure to the hospital environment and broad-spectrum antimicrobial therapy may be infected with AmpC- $\beta$-lactamase-producing strains of Enterobacter spp. or other Enterobacteriaceae. There is relatively little published experience regarding management of these organisms in patients with HA-IAI. Broad-spectrum carbapenems are generally considered the treatment of choice for patients with bacteremia and other infections because of AmpC- $\beta$-lactamase-producing gramnegative pathogens, and would likely be effective in patients with HA-IAI from these resistant strains (Table 10) [256$259,315,468]$. Cefepime has been described as an option to treat patients with these pathogens, despite the recognized propensity of other cephalosporins to serve as inducers of these enzymes [257-259,468]. Other agents, including tigecycline, aminoglycosides, and fluoroquinolones, may have activity against AmpC- $\beta$-lactamase-producing organisms, but little information is available regarding their clinical efficacy [256,257]. Ceftazidime-avibactam has good in vitro activity against AmpC- $\beta$-lactamase-producing Enterobacteriaceae $[277,469,470]$, and ceftolozane-tazobactam has activity against $70 \%$ of these strains [265], but clinical experience using these agents to treat AmpC- $\beta$-lactamaseproducing gram-negative pathogens is quite limited.

Carbapenemase-producing gram-negative bacteria present an even greater challenge to the clinician. There is a paucity of evidence regarding treatment of patients with HA-IAI who are infected with these pathogens. Most of the available literature relates to the treatment of patients with bacteremia from carbapenemase-producing Enterobacteriaceae, particularly $K$. pneumoniae. For treatment of KPC-producing strains, the combination of a carbapenem with an aminoglycoside, a polymyxin, or tigecycline has been associated with the highest efficacy, at least when the minimum inhibitory concentration (MIC) to carbapenems is not too high $[316,317,471,472]$. Ceftazidime-avibactam has activity against KPC-producing strains of Enterobacteriaceae and thus may provide an option for treating patients with HA-IAI because of this organism (Table 10) [276,469]. Treatment of patients with MBL-producing Enterobacteriaceae is more problematic, because these bacteria are resistant to nearly all $\beta$-lactam antibiotic agents, although aztreonam may be an exception. Combination therapy, using agents such as a polymyxin, tigecycline, or fosfomycin, among others, is generally advocated [434,473].

Some patients with HA-IAI may be infected with MDR, XDR, or PDR strains of gram-negative bacteria such as Pseudomonas spp. and Acinetobacter spp. [474]. These highly resistant micro-organisms may be found in patients characterized as having tertiary peritonitis $[33,87,475]$. Selection of empiric therapy in these patients is individualized, based on previous culture and susceptibility data, the patient's history of previous antimicrobial exposure, and the type of resistant pathogens encountered in the local environment. For these MDR, XDR, and PDR pathogens, combination regimens are generally used. Depending on the suspected micro-organism, this may or may not include a broad-spectrum $\beta$-lactam antibiotic agent, an aminoglycoside, a polymyxin, tigecycline (not applicable for Pseudomonas), rifampin, or fosfomycin (Table 10) [318,476-481]. 
Ceftolozane-tazobactam has activity against many MDR and XDR strains of Pseudomonas resistant to other $\beta$-lactam antibiotic agents [265,266]; ceftazidime-avibactam may also have some enhanced activity against these strains as well $[277,279]$. These newer agents, however, are not particularly active against Acinetobacter spp. Experience with these agents in critically ill patients is still quite limited. Overall, no ideal regimen has been identified for managing HA-IAI in patients infected with these highly resistant pathogens.

7.4a. We suggest that patients with HA-IAI who have received substantial previous broad-spectrum antimicrobial therapy, have had prolonged hospitalizations, have undergone multiple invasive interventions, or are known to have been colonized or infected with a resistant gram-negative organism be considered at risk for infection from a resistant gram-negative pathogen (Grade 2-B).

7.4b. We suggest use of local epidemiologic data and antibiograms for selecting empiric antimicrobial therapy of HA-IAI in patients considered at risk for infection with resistant gram-negative pathogens (Grade 2-B).

7.4c. We suggest a broad-spectrum carbapenem, with ceftolozane-tazobactam or ceftazidime-avibactam as potential alternatives, for empiric antimicrobial therapy of HA-IAI in patients considered at risk for infection with ESBLproducing Enterobacteriaceae (Grade 2-B). We suggest a broad-spectrum carbapenem, with ceftazidime-avibactam as a potential alterative, for empiric antimicrobial therapy of HA-IAI in patients considered at risk for infection with AmpC- $\beta$-lactamase-producing Enterobacteriaceae (Grade $2-B)$. We suggest combinations of a carbapenem with an aminoglycoside, a polymyxin, and/or tigecycline, or ceftazidimeavibactam as an alternative, for empiric antimicrobial therapy of HA-IAI in patients considered at risk for infection with carbapenem-resistant Enterobacteriaceae (Grade 2-B).

7.4d. We suggest combinations of a $\beta$-lactam, including ceftolozane-tazobactam, an aminoglycoside, and/or a polymyxin, for empiric antimicrobial therapy of HA-IAI in patients considered at risk for infection from MDR, XDR, or PDR strains of $\mathrm{P}$. aeruginosa (Grade 2-B). We suggest combinations of a carbapenem, an aminoglycoside, a polymyxin, and/or tigecycline for empiric antimicrobial therapy of HA-IAI in patients considered at risk for infection from $M D R, X D R$, or PDR strains of Acinetobacter spp. (Grade 2-B).

\section{E. Antifungal therapy}

Q 7.5a. Which patients with HA-IAI should be considered at risk for infection from Candida spp.?

Q 7.5b. What agents can be used for empiric antifungal therapy of HA-IAI in patients considered at risk for a candidal infection?

Risk factors for invasive candidal infections in hospitalized patients, particularly critically ill patients, include a previous surgical procedure, a history of broad-spectrum antimicrobial therapy, pancreatitis, use of parenteral nutrition, presence of invasive catheters, medical co-morbidities including diabetes mellitus, cardiac disease, renal failure, or immunosuppression, and multiple sites of colonization with Candida spp. [416,482-489]. These risk factors are generally derived from studies of patients with candidemia and may not be directly applicable to patients with HA-IAI. Studies that have more closely examined specific risk factors for Candida peritonitis have identified recurrent gastrointestinal perforations [490], upper gastrointestinal perforations [110,413,416,417], surgically treated pancreatitis [490], and previous receipt of antimicrobial therapy [107-110] as predisposing factors for HA-IAI from Candida spp. Colonization with Candida at multiple sites has been used to identify patients at high risk of an invasive candidal infection [490-493,], but the utility of this observation has been disputed [494].

Mortality rates of $20 \%-64 \%$ have been recorded in patients with HA-IAI from Candida spp., with at least some of the deaths being directly attributed to the candidal infection itself [56,109,412.413,418,483,495,496]. Delays in effective antifungal therapy have been associated with increased deaths in patients with candidemia and other invasive candidal infections, particularly those with septic shock [56,375,412,415,489].

Antifungal agents applicable to the empiric treatment of patients with HA-IAI potentially because of Candida spp. include the polyenes (amphotericin B in its various formulations), triazoles (fluconazole and voriconazole), and echinocandins (anidulafungin, caspofungin, and micafungin). Evidence from RCTs and a meta-analysis suggest that echinocandins are more efficacious than triazoles and less toxic than polyenes when treating patients with candidal infections, particularly those who are critically ill [361,362,364,365,371-373].

Although these clinical trials primarily enrolled subjects with candidemia, not Candida peritonitis, the task force believes these findings should apply to severely ill patients with HA-IAI potentially from Candida. Thus, an echinocandin is recommended for empiric treatment of these patients with HAIAI. Nonetheless, fluconazole was effective at preventing Candida peritonitis when used preemptively in patients at high risk for an infection with this organism [366]. For patients who are less critically ill, the task force suggests that empiric fluconazole should suffice in most patients. Historically, amphotericin B was the primary antifungal agent used to treat patients with intra-abdominal candidiasis; however, use of this agent has fallen into disfavor because of its toxicity [360,364]. It is not currently recommended except when other, less toxic, agents cannot be used to manage the infection [497-501].

Although C. albicans is the most common yeast found with IAI, non-C. albicans species, including $C$. glabrata, $C$. parapsilosis, and $C$. tropicalis, are isolated with increasing frequency [375,502-504]. Candida glabrata may be intrinsically resistant or demonstrate dose-dependent susceptibility to fluconazole; voriconazole has much better activity against this organism [505]. The echinocandins are highly active against nearly all Candida spp. Thus, voriconazole or an echinocandin are options in patients at risk for or known to be colonized or infected with a fluconazole-resistant species of Candida [367-369,505].

7.5a. We suggest that patients with HA-IAI because of upper gastrointestinal perforations, recurrent bowel perforations, or surgically treated pancreatitis, those who have received prolonged courses of broad-spectrum antibiotic therapy, and those who are known to be heavily colonized with Candida be considered at increased risk for infection from Candida spp. (Grade 2-B). We suggest that patients with HA-IAI found to have yeast on a Gram stain of infected peritoneal fluid or tissue be considered to have a candidal infection (Grade 2-B). 
7.5b. We recommend an echinocandin (anidulafungin, caspofungin, or micafungin) for empiric antifungal therapy of HA-IAI in severely ill patients considered at risk for infection from Candida spp. (Grade 1-B). We suggest fluconazole can be used for empiric antifungal therapy in less severely ill patients with HA-IAI considered at risk for infections from Candida spp. (Grade 2-B). We suggest an echinocandin or voriconazole for empiric antifungal therapy of patients with HA-IAI considered at risk for infection because of a fluconazole-resistant non-C. albicans strain (Grade 2-B).

\section{Timing of antimicrobial therapy}

Q 8.1. When should antimicrobial therapy be initiated in patients with a diagnosis of IAI?

Q 8.2. Should additional antimicrobial therapy be administered to patients undergoing source control procedures for IAI who are already receiving empiric antimicrobial therapy?

There is little definitive evidence on the timing of antimicrobial therapy for patients with IAI. In patients with sepsis or septic shock, a substantial proportion of whom present with IAI, several cohort and retrospective studies have demonstrated increased deaths if antimicrobial therapy is delayed [506-508]. Based on these data, the Surviving Sepsis Campaign recommends initiation of antimicrobial therapy within one hour of identifying a patient with sepsis or septic shock [6]. Nonetheless, some studies have failed to find an association between a delay of antimicrobial therapy and death for up to five hours in such patients [509-512]. Although these conflicting data question whether a one-hour time window should be considered a rigid standard for delivering antiinfective therapy once the diagnosis of IAI with sepsis has been made, the task force believes that early administration of antimicrobial agents represents a reasonable goal. It also needs to be recognized, however, that there are additional priorities in the acute management of IAI with sepsis, such as preparing the patient for definitive source control.

There are little data with regard to antimicrobial timing for patients with IAI who do not meet sepsis criteria. In general, the task force believes it is reasonable to initiate antimicrobial therapy as soon as feasible in these patients, as had been recommended in the previous guideline [4]. Withholding antimicrobial therapy until definitive peritoneal cultures can be obtained is not justified, because of the prolonged delay in effective antimicrobial therapy that could entail.

There are also important considerations with regard to timing of antimicrobial delivery relative to a source control procedure. Even though an established infection is already present in patients with IAI, the general principles of antimicrobial prophylaxis for surgical procedures still apply. Guidelines for prevention of surgical site infection specify administration of most prophylactic antibiotics within one hour of the surgical incision to ensure that adequate blood and tissue concentrations are present [238,513-515]. A number of observational studies have provided evidence in support of this recommendation [516-520].

A patient with IAI may have had an antimicrobial agent administered several hours before a source control procedure, however. Under those circumstances, adequate serum and tissue concentrations of the agent may not be present during that procedure. Therefore, the task force recommends re- administration of an anti-infective agent at the time of a source control intervention if more than two half-lives have elapsed since the previous dose. This is based on surgical site infection prevention guidelines recommending re-dosing of prophylactic agents intra-operatively if the length of the procedure exceeds two half-lives of the agent in question [238,514,521,522]. Because dissemination of pathogenic micro-organisms may occur during less-invasive source control procedures as well, it seems reasonable to adhere to this recommendation for source control interventions other than operative procedures. The task force also endorses readministration of antibiotics intra-operatively during source control if two antibiotic half-lives have elapsed since the previous dose.

8.1. We suggest initiation of empiric antimicrobial therapy within one hour or as soon as possible thereafter of the time the diagnosis of IAI is made in patients presenting with sepsis or septic shock (Grade 2-B). We also suggest initiation of antimicrobial therapy as soon as feasible in other patients with IAI, taking into account plans for subsequent source control (Grade 2-C).

8.2. We recommend re-administration of an antimicrobial agent within one hour before the start of a source control procedure for IAI if two half-lives of the agent will have passed at the time the intervention is initiated (Grade 1-B).

\section{Dosing of antimicrobial agents in adult patients}

Q 9.1. What dosages of antimicrobial agents should be used for management of IAI in adult patients?

Q 9.2. Should adjusted dosages of antimicrobial agents be used for management of IAI in adult patients who have substantial renal impairment?

Q 9.3. Should adjusted dosages of antimicrobial agents be used for management of IAI in adult patients who have substantial hepatic impairment?

Q 9.4. Should adjusted dosages of antimicrobial agents be used for management of IAI in obese patients?

Q 9.5. Should alternative dosing schedules, such as prolonged or continuous infusion of selected agents, be used for management of IAI in critically ill patients or those infected with resistant pathogens?

Standard dosing recommendations for antimicrobial agents used in the treatment of patients with IAI are listed in Table 11. These dosing recommendations have been derived from the information published in RCTs (see Supplementary Tables; see online supplementary material at www.liebertpub .com/overview/surgical-infections/53/) and supplemental publications describing individual agents, from the prescribing information for specific antimicrobials approved by the FDA [523], and from a standard pharmacology text [524]. For lower-risk patients with CA-IAI who do not have substantial renal or hepatic impairment or severe obesity, the task force believes these dosage recommendations should be fairly accurate, because they reflect dosing from clinical trials primarily enrolling lower-risk subjects without major organ impairment [3,389].

Critically ill patients may benefit from adjusted dosing of many antimicrobial agents. The evidence supporting this for patients with IAI is mostly indirect, however. Critical illness may impact the volume of distribution $(\mathrm{Vd})$ and clearance of 
Table 11. Dosing of Antimicrobial Agents

\begin{tabular}{|c|c|c|}
\hline Agent & Standard dose & Renal dose \\
\hline \multicolumn{3}{|l|}{ Aminoglycosides } \\
\hline Amikacin & $7.5 \mathrm{mg} / \mathrm{kg}$ IV q8-12h & $7.5 \mathrm{mg} / \mathrm{kg}$ IV q12-72h based on serum drug levels \\
\hline Gentamicin & $\begin{array}{l}1.5-2.5 \mathrm{mg} / \mathrm{kg} \text { IV } \\
\text { q8h-12h; } \\
4-7 \mathrm{mg} / \mathrm{kg} \text { IV q24h }\end{array}$ & $1.5-2.5 \mathrm{mg} / \mathrm{kg}$ IV q12-48h based on serum drug levels \\
\hline Tobramycin & $1.5-2 \mathrm{mg} / \mathrm{kg}$ IV q8h; $4-7 \mathrm{mg} / \mathrm{kg}$ IV q24h & $1.5-2 \mathrm{mg} / \mathrm{kg}$ IV q12-72h based on serum drug levels \\
\hline \multicolumn{3}{|c|}{ Penicillin/ $\beta$-lactamase inhibitor combinations } \\
\hline Ampicillin-sulbactam & $1.5-3 \mathrm{~g}$ IV q6h & $1.5-3 \mathrm{~g}$ IV q12-24h \\
\hline Amoxicillin-clavulanate & $1.2 \mathrm{~g} \mathrm{IV} \mathrm{q6-8h}$ & $1.2 \mathrm{~g} \mathrm{IV}$, then $0.6 \mathrm{~g} \mathrm{IV} \mathrm{q12-24h}$ \\
\hline $\begin{array}{l}\text { Ticarcillin-clavulanate } \\
\quad \text { (not currently available) }\end{array}$ & $3.1 \mathrm{~g} \mathrm{IV} \mathrm{q6h}$ & $1-2 \mathrm{~g}$ IV q12-24h \\
\hline Piperacillin-tazobactam & $3.375-4.5 \mathrm{~g}$ IV q6h & $2.25 \mathrm{~g}$ IV q6-8h \\
\hline \multicolumn{3}{|c|}{ Cephalosporins (including cephamycins) and cephalosporin- $\beta$-lactamase inhibitor combinations } \\
\hline Cefoxitin & $1-2 \mathrm{~g}$ IV q6h & $0.5-1 \mathrm{~g}$ IV q8-48h \\
\hline Cefotetan & $1-2 \mathrm{~g}$ IV q12h & $0.5-1 \mathrm{~g}$ IV q12-24h \\
\hline Cefazolin & 1-2 g IV q8h & $1 \mathrm{~g} \mathrm{q} 12-24 \mathrm{~h}$ \\
\hline Cefuroxime & $1.5 \mathrm{~g}$ IV q8h & $0.75 \mathrm{~g}$ IV q12-24h \\
\hline Cefotaxime & $1-2 \mathrm{~g}$ IV q6-8h & $0.5-1 \mathrm{~g}$ IV q8-24h \\
\hline Ceftriaxone & $1-2 \mathrm{~g}$ IV q24h & $\begin{array}{l}\text { No dosing adjustment; check blood } \\
\text { levels with dialysis }\end{array}$ \\
\hline Ceftazidime & $1-2 \mathrm{~g}$ IV q8h & $0.5-1$ g IV q12-24h \\
\hline Cefepime & $1-2 \mathrm{~g}$ IV q12h & $0.5-2 \mathrm{~g}$ IV q24h \\
\hline \multicolumn{2}{|l|}{ Cefoperazone-sulbactam: } & No dosing adjustment; maximum daily \\
\hline $1: 1$ ratio & 1-2 g IV q12h & sulbactam dosage of $1-2 \mathrm{~g}$ depending \\
\hline $2: 1$ ratio & $1.5-3 \mathrm{~g}$ IV q12h & on degree of renal impairment \\
\hline Ceftolozane-tazobactam & $1.5 \mathrm{~g}$ IV q8h & $\begin{array}{l}750 \mathrm{mg} \text { IV q8h or } 375 \mathrm{mg} \text { IV q8h depending } \\
\text { on degree of renal impairment }\end{array}$ \\
\hline Ceftazidime-avibactam & $2.5 \mathrm{~g} \mathrm{IV} \mathrm{q} 8 \mathrm{~h}$ & $\begin{array}{l}0.94-1.25 \mathrm{~g} \mathrm{IV} \mathrm{q} 8-48 \mathrm{~h} \text { depending } \\
\text { on degree of renal impairment }\end{array}$ \\
\hline $\begin{array}{l}\text { Monobactam } \\
\text { Aztreonam }\end{array}$ & $1-2 \mathrm{~g}$ IV q8h & $1-2 \mathrm{~g}$ IV, then $0.5-1 \mathrm{~g}$ IV q6-12h \\
\hline \multicolumn{3}{|l|}{ Carbapenems } \\
\hline Ertapenem & $1 \mathrm{~g} \mathrm{IV} \mathrm{q24h}$ & $0.5 \mathrm{~g} \mathrm{IV} \mathrm{q} 24 \mathrm{~h}$ \\
\hline Doripenem & $0.5 \mathrm{~g}$ IV q8h & $0.25-0.5 \mathrm{~g}$ IV q8-12h \\
\hline Imipenem-cilastatin & $0.5-1$ g IV q6-8h & $0.125-0.5 \mathrm{~g}$ IV q6-12h \\
\hline Meropenem & $1-2 \mathrm{~g} \mathrm{IV} \mathrm{q} 8 \mathrm{~h}$ & $0.5-1 \mathrm{~g}$ IV q12-24h \\
\hline \multicolumn{3}{|l|}{ Fluoroquinolones } \\
\hline Moxifloxacin & $400 \mathrm{mg}$ IV q24h & No dosing adjustment \\
\hline Ciprofloxacin & $400 \mathrm{mg}$ IV q12h & 200-400 mg IV q18-24h \\
\hline Levofloxacin & $500-750 \mathrm{mg}$ IV q24h & $250-750 \mathrm{mg}$ IV q24-48h \\
\hline Glycylcycline & $100 \mathrm{mg}$ IV, then $50 \mathrm{mg}$ IV q12h & No dosing adjustment \\
\hline \multicolumn{3}{|l|}{ Anti-anaerobic agents } \\
\hline Clindamycin & 600-900 mg IV q6-12h & No dosing adjustment \\
\hline Metronidazole & $1 \mathrm{~g}$ IV, then $0.5 \mathrm{~g}$ IV q6-8h & No dosing adjustment \\
\hline \multicolumn{3}{|c|}{ Agents with activity against gram-positive organisms } \\
\hline Ampicillin & $1-2 \mathrm{~g}$ IV q4-6h & $\begin{array}{l}\text { Reduce frequency of dosing to } \mathrm{q} 6 \mathrm{~h}-\mathrm{q} 24 \mathrm{~h} \\
\text { depending on degree of renal impairment }\end{array}$ \\
\hline Daptomycin & $4-6 \mathrm{mg} / \mathrm{kg} \mathrm{q} 24 \mathrm{~h}$ & $4-6 \mathrm{mg} / \mathrm{kg} \mathrm{q} 48 \mathrm{~h}$ \\
\hline Linezolid & $600 \mathrm{mg}$ IV q12h & No dosing adjustment \\
\hline Teicoplanin & $\begin{array}{l}400 \mathrm{mg} \text { IV } \mathrm{q} 12 \mathrm{~h} \times 3 \text { doses, } \\
\text { then } 400 \mathrm{mg} \text { IV q24h }\end{array}$ & $\begin{array}{l}\text { Reduction of daily dosage to one-half to one-third } \\
\text { depending on degree of renal impairment }\end{array}$ \\
\hline Vancomycin & $10-20$ mg/kg IV q12h & $\begin{array}{l}10-20 \mathrm{mg} / \mathrm{kg} \text { IV q24h with subsequent dosing } \\
\text { based on serum drug levels }\end{array}$ \\
\hline \multicolumn{3}{|l|}{ Antifungal agents: Azoles } \\
\hline Fluconazole & $400 \mathrm{mg}$ IV q24h, then $200 \mathrm{mg}$ IV q24h & $\begin{array}{l}200 \mathrm{mg} \text { IV q24h, then } 100 \mathrm{mg} \text { IV q24h } \\
\text { or after hemodialysis }\end{array}$ \\
\hline Voriconazole & $\begin{array}{l}6 \mathrm{mg} / \mathrm{kg} \mathrm{q} 12 \mathrm{~h} \times 2 \text { doses, } \\
\text { then } 3-4 \mathrm{mg} / \mathrm{kg} \mathrm{q} 12 \mathrm{~h}\end{array}$ & $\begin{array}{l}\text { IV formulation not recommended because of } \\
\text { accumulation of SBECD (sulfobutyl ether } \\
\text { beta-cyclodextrin sodium) vehicle }\end{array}$ \\
\hline \multicolumn{3}{|l|}{ Antifungal agents: Echinocandins } \\
\hline Anidulafungin & $200 \mathrm{mg}$ IV, then $100 \mathrm{mg}$ IV q24h & No dosing adjustment \\
\hline Caspofungin & $70 \mathrm{mg}$ IV, then $50 \mathrm{mg}$ IV q $24 \mathrm{~h}$ & No dosing adjustment \\
\hline Micafungin & $100 \mathrm{mg}$ IV q24h & No dosing adjustment \\
\hline \multicolumn{3}{|l|}{ Antifungal agents: Polyenes } \\
\hline Amphotericin B deoxycholate & $1-1.5 \mathrm{mg} / \mathrm{kg}$ IV q24h & $1-1.5 \mathrm{mg} / \mathrm{kg}$ IV q48h \\
\hline $\begin{array}{l}\text { Amphotericin B lipid complex, } \\
\text { liposomal amphotericin B }\end{array}$ & $5 \mathrm{mg} / \mathrm{kg}$ IV q24h & No dosing adjustment \\
\hline
\end{tabular}

$\mathrm{IV}=$ intravenous. 
antimicrobial agents. The Vd for hydrophilic antibiotic agents, such as $\beta$-lactams, aminoglycosides, and glycopeptides, may be expanded as a result of administration of large volumes of IV fluids, and further exacerbated by fluid shifts into the extravascular space [525-527]. Increased renal clearance of antibiotic agents may also occur in some critically ill patients [525,526,528]. These alterations could lead to suboptimal blood or tissue concentrations, with the potential for treatment failure if the infection is because of less susceptible pathogens [525-529]. For $\beta$-lactams, aminoglycosides, tigecycline, vancomycin, and polymyxins, some clinical studies have found improved outcomes in selected critically ill patients treated with higher dosages or shorter dosing intervals, particularly in those patients infected with less susceptible organisms [478480,525,526,530,531].

Thus, the task force believes that altered antimicrobial dosing regimens, including higher initial loading doses, may be useful in selected patients with IAI. Critically ill patients, however, may also have impaired renal function, leading to decreased antibiotic clearance, and a risk for antibiotic toxicity [525-528,532]. Therefore, use of higher dosages or altered dosing intervals in critically ill patients with IAI needs to be individualized.

Dosing adjustments of antimicrobial agents cleared primarily by renal elimination are frequently necessary in patients with IAI who have impaired kidney function. Most antimicrobial agents do not require dosage adjustments until the glomerular filtration rate (GFR) decreases below $60 \mathrm{~mL} / \mathrm{min}$. The GFR is typically estimated according to the Cockcroft-Gault formula $[533,534]$, which relies on serum creatinine measurements. Other methods for determining estimated GFR (eGFR) are available, which may be preferable to the Cockcroft-Gault method; however, these also lack accuracy and precision, particularly in critically ill patients [535-537]. Because the eGFR heavily influences dosing of antimicrobial agents in patients with renal impairment, the treating clinician should be aware of pitfalls associated with these calculations of eGFR.

In general, the initial dosage of most antimicrobial agents should not be altered in patients with impaired renal function, because initial drug concentrations will primarily reflect $\mathrm{Vd}$ and not clearance [526,527,538,539]. Subsequent dosing of antimicrobial agents will typically be altered according to the eGFR [539]. Table 11 provides general recommendations for antimicrobial dosing adjustments in patients with impaired kidney function [523,526,539].

Antimicrobial dosing adjustments in patients with renal dysfunction can be expedited using therapeutic drug moni- toring [527,539]. Commercial assays are commonly available for the measurement of blood concentrations of vancomycin and aminoglycosides, although not for most other antibiotic agents. Therapeutic drug monitoring may be of particular value when the GFR fluctuates during the course of antimicrobial therapy. Antimicrobial dosing in patients with renal dysfunction can also be facilitated by consultation with a clinical pharmacist familiar with use of antimicrobial agents. This may be particularly important in patients undergoing various forms of renal replacement therapy, such as intermittent hemodialysis or continuous veno-venous hemofiltration-dialysis. Specific recommendations for antimicrobial dosing in patients undergoing renal replacement therapy are beyond the scope of this guideline, but several reviews provide information on this subject [527,536,538-540].

There is far less information available regarding antimicrobial dosage adjustments in patients with hepatic dysfunction. Liver disease has an impact primarily on the dosing of antibiotic agents cleared by hepatic metabolism; thus, hepatic dysfunction can lead to accumulation of certain anti-infective agents and a risk of associated toxicity [528,541,542]. Table 12 summarizes recommendations made by Halilovic and Heintz [542] for dosing of some hepatically cleared antibiotic agents used in the treatment of patents with IAI.

Alternative dosing regimens for obese patients should also be considered. Obesity may have a major impact on the $\mathrm{Vd}$ of antimicrobial agents. In general, the $\mathrm{Vd}$ of hydrophilic antibiotic agents correlates better with the lean body mass or ideal body weight, whereas the $\mathrm{Vd}$ of lipophilic antibiotic agents correlates better with total body weight. For many antibiotic agents of interest, an adjusted body weight calculation, which adds a percentage of the excess body weight to the ideal body weight, has been used to predict Vd [543-548]. Obese patients with a higher Vd may benefit from a higher initial dose of a given antimicrobial agent.

Renal clearance of antibiotic agents can also be affected by obesity. In the absence of intrinsic renal disease, obesity generally produces an increase in creatinine clearance. Standard equations for creatinine clearance, however, such as the Cockcroft-Gault formula [533], may produce misleading values [545,549], and corrective formulas, such as the Salazar-Corcoran equations [550], have not been fully validated [551]. Calculations of eGFR may be particularly prone to error in critically ill obese patients [535,543,545], making dosage adjustments in these patients quite problematic.

Recommendations based on pharmacokinetic principles have been developed for the dosing of individual antibiotic

Table 12. Antimicrobial Dosage Adjustments in Patients with Cirrhosis

\begin{tabular}{ll}
\hline Agent & \\
\hline Cefotaxime & No dosage adjustment \\
Ceftriaxone & Consider $50 \%$ reduction in patients with Child-Pugh class C cirrhosis \\
Moxifloxacin & No dosage adjustment \\
Ciprofloxacin & No dosage adjustment \\
Tigecycline & Decrease maintenance dosage to $25 \mathrm{mg}$ q12h in patients with Child-Pugh class C cirrhosis; \\
& no change in initial loading dosage \\
Metronidazole & Decrease dosage to $500 \mathrm{mg}$ q12-24h in patients with Child-Pugh class A, B, or C cirrhosis \\
Clindamycin & Decrease dosage by $50 \%$ in patients with Child-Pugh class C cirrhosis \\
Linezolid & No dosage adjustment; use cautiously in patients with Child-Pugh class C cirrhosis \\
\hline
\end{tabular}


Table 13. Antimicrobial Dosage Adjustments in Obese Patients

\begin{tabular}{|c|c|}
\hline Agent & Suggested Adjustment \\
\hline Aminoglycosides & $\begin{array}{l}\text { Base dosage on adjusted body weight (ideal body weight plus } 40 \% \text { of the excess weight } \\
\text { above ideal weight) }\end{array}$ \\
\hline $\begin{array}{l}\beta \text {-lactam } / \beta \text {-lactamase } \\
\text { inhibitor combination }\end{array}$ & $\begin{array}{l}\text { Use dosages at the higher end of treatment range, or use standard dosages with therapeutic } \\
\text { drug monitoring in critically ill obese patients }\end{array}$ \\
\hline Cephalosporin & $\begin{array}{l}\text { Use of dosages at the higher end of treatment ranges, or use standard dosages with } \\
\text { therapeutic drug monitoring in critically ill obese patients }\end{array}$ \\
\hline Fluoroquinolones & $\begin{array}{l}\text { Use dosages at the higher end of treatment ranges, or base dosage on adjusted body weight } \\
\text { (ideal body weight plus } 45 \% \text { of the excess over ideal body weight) }\end{array}$ \\
\hline Clindamycin & Use higher dosages up to $4.8 \mathrm{~g} / \mathrm{d}$ in patients with a body mass index greater than $40 \mathrm{~kg} / \mathrm{m}^{2}$ \\
\hline Vancomycin & Base dosage on actual body weight, with limitation of the total dose to $4 \mathrm{~g} / \mathrm{d}$ \\
\hline Linezolid & Dosage adjustment for obesity not currently recommended \\
\hline Daptomycin & Base dosage on total body weight \\
\hline
\end{tabular}

agents or classes in obese patients. These include aminoglycosides [543-545,548,552-556], various $\beta$-lactam antibiotic agents [531,543-546,556-563], fluoroquinolones [543,544,548,556,564-569], clindamycin [548,569], vancomycin [344,452,544,545,548,556], linezolid [570,571], and daptomycin [545,548,556,572,573]. Suggestions for dosing of these antibiotic agents in obese patients are summarized in Table 13. As of yet, however, there are little data documenting improvements in clinical outcomes with use of these alternative dosing strategies. In fact, obesity has not been uniformly identified as a significant risk factor for an adverse outcome in patients with IAI [574,575],

Continuous or prolonged (over 3-4 h) antibiotic infusion, as opposed to intermittent infusion (over 30-60 min), is a therapeutic tactic designed to take advantage of the pharmacokinetic and pharmacodynamic properties of specific antibiotic agents to maximize their clinical efficacy. The primary class of antibiotic agents that has been assessed using continuous or prolonged infusion are $\beta$-lactams. The main pharmacodynamic parameter believed to be important in a bacteriologic response to $\beta$-lactams is the time that the local concentration is above the MIC of the specific bacterium (ideally, $40 \%$ or more of the dosing interval). This time is enhanced by continuous or prolonged infusion [576-580]. One study in patients with IAI found that continuous infusion of cefotaxime resulted in peritoneal fluid concentrations of the antibiotic at least five times greater than the MICs of the infecting Enterobacteriaceae [581].

Meta-analyses comparing continuous or prolonged antibiotic infusion to conventional intermittent infusion in a broad range of patients with various types of infections have come to conflicting conclusions with regard to the efficacy of this approach, however [579,580,582]. A recent large RCT of patients with severe sepsis, $25 \%$ of whom had an abdominal source, did not demonstrate any advantage to continuous infusion; results in patients with IAI were not reported separately [583]. A single RCT comparing continuous versus intermittent infusion of piperacillin-tazobactam in patients with IAI (Supplementary Table P; see online supplementary material at www.liebertpub.com/overview/surgical-infections/ 53/) also did not demonstrate any advantage to the continuous infusion approach [584]. A separate analysis of pharmacokinetic and pharmacodynamic parameters and the MICs of the isolated pathogens in this trial suggested that either ap- proach would likely have sufficed in these patients, however [585]. Thus, while continuous or prolonged antibiotic infusion appears safe and effective for patients with IAI, it remains unknown if there is a demonstrable clinical benefit to its use. The task force believes that this approach could be considered when treating potentially less susceptible strains of gram-negative pathogens in critically ill patients with IAI.

9.1. We recommend use of standard dosages of antimicrobial agents for lower-risk patients with CA-IAI who are not severely obese and who do not have substantial renal or hepatic impairment (Grade 1-B). We suggest use of higher dosages of antimicrobial agents for selected higher-risk patients with CA-IAI or HA-IAI (Grade 2-B).

9.2. We suggest use of adjusted dosages of antimicrobial agents, based on available clinical outcome data, standard pharmacokinetic parameters, and therapeutic drug monitoring, where applicable, for management of IAI in patients with significant renal impairment (Grade 2-B).

9.3. We suggest use of adjusted dosages of selected, hepatically cleared antimicrobial agents for management of IAI in patients who have substantial hepatic impairment (Grade 2-B).

9.4. We suggest use of adjusted dosages of selected antimicrobial agents, based on available clinical outcome data and pharmacokinetic parameters, for management of IAI in obese patients (Grade 2-B).

9.5. We suggest that prolonged or continuous infusion of $\beta$ lactam antibiotic agents does not need to be used routinely for treatment of patients with IAI, but is an option for management of IAI in critically ill patients and those at risk for infection with resistant gram-negative pathogens (Grade 2-B).

\section{Duration of antimicrobial therapy}

Q 10.1a. Should antibiotic agents be given to patients with severe or necrotizing pancreatitis who do not have a documented peri-pancreatic infection?

Q 10.1b. Should antibiotic agents be given to patients with uncomplicated acute colonic diverticulitis?

Q 10.2. Which patients should be considered to have intra-abdominal contamination only, and receive antimicrobial therapy for no greater than 24 hours?

Q 10.3a. What should the duration of antimicrobial therapy be for patients with established IAI? 
Q 10.3b. What should the duration of antimicrobial therapy be for patients with established IAI in whom a definitive source control was not performed?

Q 10.4. Should patients with secondary bacteremia from IAI receive a longer course of antimicrobial therapy?

Q 10.5. Should immunosuppressed patients with IAI receive longer courses of antimicrobial therapy?

Limiting the duration of antimicrobial therapy in patients with IAI and other infections is an important antimicrobial stewardship measure. Short duration therapy should decrease the development of antimicrobial resistance as well as lessen the chance that the patient will have an adverse reaction to an antimicrobial agent [197,529,586]. Antimicrobial agents have been given to patients with or at risk for IAI for a number of reasons, including prevention of infection in patients with a non-infectious inflammatory process such as acute pancreatitis, peri-operatively for an uncomplicated IAI such as non-perforated appendicitis, for intra-abdominal contamination that has occurred as a result of trauma or iatrogenic injury, or for an established complicated IAI.

The use of antibiotic agents to prevent infection in patients with severe or necrotizing pancreatitis had been advocated previously. Six RCTs of antibiotic prophylaxis for patients with severe pancreatitis have been published since 2000 (Supplementary Table Q; see online supplementary material at www.liebertpub.com/overview/surgical-infections/53/) [587-592]. None showed any impact on death or on most measures of morbidity, although two showed a decrease in the incidence of infection, mainly extra-pancreatic infections $[588,590]$. Recent meta-analyses of these and earlier studies have, for the most part, demonstrated little benefit to the use of antimicrobial agents in patients with severe or necrotizing pancreatitis who do not have an established infection (Supplementary Table R; see online supplementary material at www .liebertpub.com/overview/surgical-infections/53/) [593-598].

One meta-analysis showed a survival benefit with use of prophylactic antibiotic agents, but only if cohort studies were included along with RCTs [599]. Other meta-analyses have suggested some reductions in rates of secondary pancreatic infection, need for operative intervention, occurrence of nonpancreatic infections, or hospital length of stay, but without changes in deaths or other global outcomes [600-603]. These benefits, however, were not detected when analyzing only higher quality or more recent studies [598,604]. Concerns have also been raised about increased bacterial resistance as a result of treating patients with severe pancreatitis with prophylactic antimicrobials $[109,588,589]$. Based on the lack of clear efficacy and the potential risk of increased bacterial resistance, the task force recommends against use of antibiotic agents to prevent infection in patients with severe or necrotizing pancreatitis.

The need for antibiotic agents in the management of uncomplicated acute sigmoid diverticulitis has come into question. Two retrospective studies found that many patients with acute colonic diverticulitis could be treated entirely without antibiotic agents $[605,606]$. A subsequent large RCT of this hypothesis (Supplementary Table S; see online supplementary material at www.liebertpub.com/overview/ surgical-infections/53/) found no differences in outcomes in patients with uncomplicated, left-sided diverticulitis who received antibiotic agents and IV hydration versus those who received IV hydration alone [607]. A recent Cochrane review suggests that antibiotic agents may not be necessary, although calling for additional high quality trials [608]. Thus, omitting use of antimicrobial therapy in selected lower-risk patients with acute, uncomplicated sigmoid diverticulitis appears to be a reasonable option. It would be premature to conclude, however, that this approach should be used in higher-risk patients, such as those receiving immunosuppressive therapy.

Many patients, such as patients with penetrating abdominal trauma, present with intra-abdominal contamination without having an established IAI at the time of a surgical procedure. Antimicrobial use for such patients falls into a gray area between surgical prophylaxis and treatment for IAI. Guidelines for surgical prophylaxis generally recommend no more than 24 hours of antibiotic therapy to prevent surgical site infection [238,515]; in fact, the need for any postoperative antibiotic administration has been questioned $[609,610]$.

Several lines of evidence support limiting peri-operative antibiotic therapy to no more than 24 hours in patients with intra-abdominal contamination, even though such patients are at increased risk for infection. In three RCTs included in the previous evidence review [3], infectious outcomes were similar in patients with abdominal contamination as a result of traumatic injury treated with 24 hours compared with five days of antibiotic therapy post-operatively [611-613]. The duration of intra-abdominal contamination previously defined as differentiating contamination from an established infection was 24 hours for an upper gastrointestinal (stomach, duodenal, biliary) source and 12 hours for a lower gastrointestinal (small bowel, colon) source $[2,4]$. There is also evidence that post-operative antibiotic therapy can be limited to no more than $24 \mathrm{~h}$ in patients with uncomplicated IAI.

Recent RCTs (Supplementary Table T; see online supplementary material at www.liebertpub.com/overview/ surgical-infections/53/) have found no benefit to more than 24 hours of antibiotic therapy in patients with non-perforated appendicitis and patients undergoing cholecystectomy for acute cholecystitis [614,615]. Older, allocation-based studies reviewed previously [3] demonstrated similar rates of postoperative infection in patients with non-perforated or ischemic gastrointestinal processes treated with peri-operative antibiotic agents only compared with historical controls receiving more prolonged courses of therapy [616,617]. Therefore, for most patients undergoing source control for intra-abdominal contamination without an established infection or for an uncomplicated IAI in which the source control procedure can completely eradicate the infection, the task force concluded that antimicrobial use should be limited to no more than 24 hours.

There are increasingly strong data that duration of antimicrobial therapy for patients with established IAI can be somewhat shorter than had been used in the past. Older studies suggested no differences in outcome comparing five days with longer courses of antimicrobial therapy for patients with gastrointestinal perforations who had adequate source control [616-618]. More recent retrospective cohort studies have also found no significant differences in the rates of postoperative infections in patients with complicated appendicitis who received shorter duration of antimicrobial therapy 
(generally less than five days) versus longer duration therapy (five days or more) $[619,620]$. In fact, some retrospective studies suggested that patients receiving prolonged antimicrobial therapy for IAI were at increased risk of secondary infections and death [621,622].

Two recent RCTs (Supplementary Table U, see online supplementary material at www.liebertpub.com/overview/ surgical-infections/53/) also support a decreased duration of therapy for IAI. A smaller study found three days of antibiotic therapy was as effective as five or more days of therapy in patients with mild to moderate IAI, $50 \%$ of whom had perforated appendicitis [623]. A larger RCT supported by the SIS (the STOP-IT trial) demonstrated that a fixed four-day course of antimicrobial therapy was as effective as a longer, symptom-based duration of therapy (average of eight days of antimicrobial therapy). Importantly, this latter trial restricted the number of patients with complicated appendicitis to $15 \%$. In addition, the mean APACHE II score was approximately 10, suggesting the patients in this trial were more severely ill than those in many prospective trials of antimicrobial therapy that have used a longer duration of therapy [82]. Overall, the results of this and other studies suggest that a 4-day duration of therapy is adequate for most patients with IAI.

The duration of antimicrobial therapy for patients with IAI treated without a source control procedure (such as those with complicated diverticulitis or a peri-appendiceal phlegmon managed non-operatively) is open to question. Most patients selected for non-operative management are likely lower-risk patients who have robust host defenses, which allows them to locally control the infection. There are very little data on standardized approaches to antimicrobial therapy in such patients; even when antibiotic duration is described, the rationale for a given duration is rarely discussed. Previous studies have shown that resolution of fever, leukocytosis, and paralytic ileus is associated with a high likelihood of treatment success in patients with IAI who undergo definitive source control $[624,625]$, making it reasonable to discontinue anti-infective agents at that time. The consensus of the task force was that lower risk-patients who did not undergo definitive source control could have antimicrobial therapy discontinued at the time of resolution of these symptoms and did not have to undergo a prolonged course of therapy. If signs of infection persisted after five to seven days, however, those patients should be considered to have had treatment failure and undergo imaging and other studies to determine whether or not a definitive source control should be undertaken.

There is a wide variation in the duration of antimicrobial therapy for treatment of patients with secondary bacteremia, including that from an abdominal source [626]. Recent studies suggest that patients with transient secondary blood stream infections do not need more than seven days of antibiotic treatment. One study of critically ill patients with secondary blood stream infections, including those from a peritoneal source, found no difference in outcomes among patients receiving shorter (seven days or fewer) versus longer courses of antimicrobial therapy [627]. A systematic review and meta-analysis of duration of antibiotic therapy among critically ill patients with secondary bacteremia also found that patients who received shorter courses of anti-infective therapy, generally defined as seven or fewer days, had out- comes similar to patients receiving longer courses of therapy [628]. Thus, the task force has suggested that patients with transient bacteremia because of IAI can have antimicrobial therapy limited to seven days.

There are some patients with IAI for whom shorter-course antimicrobial therapy might not necessarily be appropriate. One group of patients at higher risk for treatment failure are those who receive immunosuppressive medications, such as patients who have undergone solid organ transplant procedures. These patients are frequently treated with prolonged antimicrobial therapy for any infection, including IAI. Definitive data are not available to draw any conclusions as to the efficacy of shorter courses of antimicrobial therapy in these patients. It is also uncertain if short course therapy should be used in critically ill patients with severe sepsis and septic shock. Although the STOP-IT trial did not demonstrate any benefit of prolonged antimicrobial therapy in any subgroup of patients, including those with an APACHE II score greater than 10 [82], relatively few severely ill patients with sepsis or septic shock were enrolled in the trial. There is anecdotal evidence that stopping antimicrobial therapy in critically ill patients with uncontrolled tertiary peritonitis may lead to increased deaths [629]; however, such patients may actually be experiencing treatment failure, for which further antiinfective treatment would generally be warranted. Overall, the task force has concluded that shorter course antimicrobial therapy can be used in most patients with IAI, but that some caution may be needed when applying this principle to immunosuppressed patients or those with ongoing signs of sepsis or septic shock.

10.1a. We recommend against the use of antibiotic agents to prevent infection in patients with severe or necrotizing pancreatitis (Grade 1-B).

10.1b. We suggest that antibiotic therapy may not be necessary for the treatment of lower-risk patients with uncomplicated acute colonic diverticulitis (Grade 2-B).

10.2. We recommend that antimicrobial therapy be limited to 24 hours in patients with traumatic bowel perforations operated on within 12 hours (Grade 1-A), patients with gastroduodenal perforations operated on within 24 hours (Grade 1-C), patients with acute or gangrenous appendicitis in the absence of perforation (Grade 1-A), patients with acute or gangrenous cholecystitis in the absence of perforation (Grade 1-A), or patients with ischemic, non-perforated bowel (Grade 1-C).

10.3a. We recommend no more than four full days (96 h) of antimicrobial therapy for patients with IAI who had an adequate source control procedure (Grade 1-A).

10.3b. We suggest that no more than 5-7 days of antimicrobial therapy be provided to patients with established IAI in whom a definitive source control procedure is not performed. We suggest that clinical parameters, including fever, leukocytosis, and adequacy of gastrointestinal function, be assessed periodically to determine whether antimicrobial therapy can be discontinued sooner. We suggest that patients who do not respond fully to antimicrobial therapy within 57 days be reassessed for a potential source control intervention (Grade 2-C).

10.4. We suggest that most patients with secondary bacteremia because of IAI who have undergone adequate source control and are no longer bacteremic can have antimicrobial therapy discontinued after seven days (Grade 2-B). 
10.5. There are insufficient data to evaluate duration of therapy in patients receiving immunosuppressive medications (no recommendation).

\section{Pathogen-directed antimicrobial therapy}

Q 11.1a. Should lower-risk patients with CA-IAI have antimicrobial therapy changed on the basis of culture results?

Q 11.1b. Should higher-risk patients with CA-IAI and those with HA-IAI have antimicrobial therapy changed on the basis of culture results?

Q 11.1c. Can treatment of patients with highly resistant organisms, isolated as part of a mixed peritoneal culture in patients with HA-IAI, be deferred?

Q 11.2. Should antimicrobial therapy be de-escalated or streamlined on the basis of culture results in patients with IAI?

The use of culture data to guide antimicrobial therapy is an established principle for the treatment of patients with infectious diseases $[6,197,200]$. It is unclear, however, whether this principle should be applied routinely to patients with IAI. Indirect evidence provides little support for routine pathogendirected therapy in lower-risk patients with CA-IAI. As discussed in Section 3, routine cultures in these patients have been found to provide little benefit, suggesting that altering therapy on the basis of those results would have little impact [186,188-191,630]. Further, recent RCTs have not identified higher failure rates in lower-risk patients who receive a narrower-spectrum versus a broader-spectrum antimicrobial regimen, despite the occasional isolation of micro-organisms resistant to a narrower-spectrum agent [215,225,226,228230,249-253,294,295,300]. Finally, the use of shorter courses of antimicrobial therapy [82,623], as recommended in the previous Section, would make use of pathogen-directed therapy moot in many lower-risk patients with IAI. Therefore, the task force does not believe that routine pathogendirected antimicrobial therapy is necessary in lower-risk patients who are making a satisfactory response to empiric therapy. Alteration of the antimicrobial regimen is appropriate, however, in patients who do not respond to initial therapy and are classified as having treatment failure, discussed further in Section 12.

There may be a greater rationale for providing pathogendirected therapy to higher-risk patients with CA-IAI and HAIAI. Altering an initially inadequate antimicrobial regimen according to culture results appeared to be important in patients with pneumonia or bacteremia, although results were not as good in those patients as they were in those whose initial empiric regimen provided adequate coverage $[197,199,200]$. There is relatively little direct evidence as to whether this approach also applies to higher-risk patients with IAI. One retrospective study found that altering an initially inadequate antimicrobial regimen did not improve outcomes in patients with IAI, although not all of the patients in the study would be considered at higher risk [49]. Nonetheless, based on the potential deleterious consequences of inadequate antimicrobial therapy in higher-risk patients, the task force believes it is reasonable to adjust that therapy on the basis of culture results in higher-risk patients with CA-IAI or HA-IAI. As with lower-risk patients, however, this is probably unnecessary in patients who are nearing completion of a course of therapy and have already exhibited satisfactory clinical responses.

Peritoneal cultures from some higher-risk patients may reveal a highly resistant micro-organism along with larger numbers of less resistant pathogens. It is uncertain whether a definitive antimicrobial regimen must be active against all of the isolated micro-organisms or just against the predominant ones. Published information specifically examining this question does not exist. The task force has therefore not made any recommendation on this question.

De-escalation or streamlining of antimicrobial therapy may be an option for higher-risk patients with CA-IAI and those with HA-IAI once definitive culture results are available. Deescalation may include conversion from a broader-spectrum to a narrower-spectrum antibiotic agent, eliminating duplicative agents if a single agent will suffice to manage the isolated organisms, and discontinuation of agents effective against gram-positive, gram-negative, or fungal pathogen if those micro-organisms are not isolated. In selected patients, changing to an oral rather than an IV agent may also be an option [197]. Several studies have described de-escalation in critically ill patients with sepsis from an abdominal source, although not all have reported the results in patients with IAI separately [146,198,631-636]. Importantly, none of these studies have identified any adverse effects of de-escalation, and some have suggested decreased deaths in patients who underwent deescalation [146,198,635]. Based on the available data, the task force recommends that de-escalation or streamlining of antimicrobial therapy be employed whenever feasible.

11.1a. We recommend against changing antimicrobial therapy on the basis of culture results in lower-risk patients with CA-IAI who have a satisfactory clinical response to source control and empiric antimicrobial therapy (Grade $1-B)$.

11.1b. We suggest modifying antimicrobial therapy in higher-risk patients with CA-IAI and HA-IAI if culture results identified organisms resistant to the initial empiric regimen and further antimicrobial therapy is planned (Grade 2-C).

11.1c. There are insufficient data to make any recommendation regarding modification of antimicrobial therapy in patients with HA-IAI who have a highly resistant organism isolated as a minor component of a mixed peritoneal culture (no recommendation).

11.2. We recommend routinely de-escalating or streamlining antimicrobial therapy in higher-risk patients with CAIAI and HA-IAI to the narrowest-spectrum agent or agents having activity against the isolated micro-organisms, once definitive culture results are available (Grade 1-B).

\section{Treatment failure}

\section{A. Source control for treatment failure}

$\mathrm{Q}$ 12.1a. What are the clinical indicators of source control failure in patients with IAI?

Q 12.1b. When should patients with IAI be assessed for source control failure?

$Q$ 12.1c. What diagnostic maneuvers should be undertaken in patients who are suspected of having source control failure?

Q 12.1d. What therapeutic approach should be undertaken with patients with failure of initial source control? 
$\mathrm{Q}$ 12.1e. When should further source control procedures be undertaken in patients considered to have a treatment failure?

Q 12.1f. Should abdominal fluid or tissue cultures be obtained in patients with treatment failure?

After initial source control, the clinician may be faced with the task of recognizing and developing a therapeutic plan for patients who fail that intervention. The diagnosis of source control failure may be problematic. Symptoms and signs of recurrent or ongoing IAI, such as abdominal pain, rebound tenderness, ileus, fever, or leukocytosis, are not specific, being quite common in all post-operative patients. Further, these signs may not be present in some patients with recurrent or ongoing IAI. Use of risk factors to identify patients with source control failure, such as those outlined in Section 1, are also insufficient to make a diagnosis in patients with source control failure. Source control failure will actually develop in only a fraction of these higher-risk patients, particularly when the initial procedure was considered adequate [172].

Several studies have demonstrated that changes in physiologic parameters and other clinical problems that become manifest at or beyond post-operative day two are far more predictive of source control failure than the patient status at the time of the initial source control procedure. Adverse trends in individual measures of heart rate, temperature, the ratio of the arterial partial pressure of oxygen to the fractional inspired oxygen concentration $\left(\mathrm{PaO}_{2}: \mathrm{F}_{\mathrm{I}} \mathrm{O}_{2}\right)$, C-reactive protein level, procalcitonin level, SAPS II or multiple organ failure (MOF) or multiple organ dysfunction syndrome (MODS) scores, the development of a fascial dehiscence, or the finding of inadequacy of empiric antimicrobial coverage are all associated with a higher risk of source control failure $[15,32,50,57,70,81,637,638]$. Thus, the task force would recommend using these temporal signals to help identify patients with a high likelihood of failed source control.

Models combining trends in various risk factors or physiologic scores may have greater potential for predicting source control failure, although none of the current models provide high certainty. Serial MODS scores were used as triggers for on-demand re-laparotomy in one RCT, but there was still a relatively high $31 \%$ rate of negative re-laparotomy [170]. A related study found that the combination of changes in several physiologic parameters predicted a positive relaparotomy, but even the best model had an overall accuracy of approximately $80 \%$ [57]. Thus, although the decision to intervene in a patient with presumed source control failure may be aided by use of such models, clinical acumen still needs to be exercised to accurately identify patients with source control failure.

Death is significantly higher in patients with IAI who have a failure of the index source control procedure [48,51,61,71, $84,126,637,639]$. Moreover, the time to re-operation may be a further determinate of outcome, with therapeutic delays greater than 24-48 hours being associated with increased deaths $[51,81]$. Thus, early identification of the patient with source control failure through diagnostic imaging or relaparotomy would be beneficial. Negative diagnostic studies or re-laparotomies, however, may lead to complications as well as increases in resource utilization. The opinion of the task force is that diagnostic investigation or therapeutic interventions should be strongly considered in patients who have progressive deterioration or no improvement in inflammatory markers or signs of organ function 48 hours after the index intervention, and in those patients who show evidence of ongoing inflammation 5-7 days after the index procedure.

In most patients, the imaging study of choice to investigate treatment failure is contrast-enhanced computed tomographic (CT) imaging [640-642]. The CT scans are generally quite sensitive in detecting infected intra-abdominal fluid collections, particularly when compared with physical examination, laboratory studies, and other imaging modalities [643-645]. The CT scans, however, do not necessarily differentiate infected from uninfected post-operative fluid collections [646-648]. The CT scans may also be less useful for detecting infections because of early anastomotic leaks $[649,650]$. It has generally been accepted that the utility of contrast-enhanced CT scans increases the longer it is after initial source control. One report suggested, however, that a good diagnostic yield is obtained even at 3-7 days after the index procedure with current generation CT scanners [651]. Nonetheless, because of the risk of false negative imaging in patients with IAI who have early treatment failure, excessive reliance should not be placed solely on CT scanning to determine the need for re-intervention.

Optimal re-intervention in patients with source control failure, as with the index source control procedure, should include measures directed at the elimination, drainage, or control of infected fluid and tissue collections and the prevention of ongoing contamination. Operative morbidity, however, is generally high in patients with source control failure, particularly in those who have already undergone extensive abdominal operations. Thus, when feasible, use of less invasive procedures should be considered. For localized fluid collections, percutaneous drainage is generally recommended [128,149]. Even when complete source elimination is not technically possible, however, less invasive techniques may still be beneficial as temporizing measures, as has been demonstrated for patients with widespread infected pancreatitis [143-145,652]. Overall, the task force supports the concept that some attempt at source control should be undertaken in patients with recurrent or ongoing IAI, because outcomes in these patients are poor in the absence of source control, even with continuation of antimicrobial therapy. The nature of that source control procedure, however, needs to be individually tailored to the patient, taking into consideration both the risks and benefits of the proposed intervention.

There are scant data regarding timing of source control for IAI in patients with treatment failure. The task force believes that undertaking source control within 24 hours of diagnosis is appropriate based on the limited data available $[49,146]$. In critically ill patients with treatment failure following an index procedure, however, the time to re-operation appears to be a determinate of outcome $[51,81,147,148]$. Thus, when treatment failure is established in a critically ill patient, efforts to achieve source control should be undertaken as expeditiously as possible.

Patients with source control failure are typically at risk for infections with more resistant pathogens than those who have CA-IAI and may be at risk for highly resistant micro-organisms if they have had significant exposure to the healthcare environment and particularly previous antimicrobial therapy 
[17,22-24,26,27,32,43,62,86,88,89]. Inadequate empiric antimicrobial therapy has been independently associated with death in these patients [43,54,58,187,193-195]. Thus, there is a rationale for obtaining peritoneal cultures in patients with source control failure, just as there is generally for any higherrisk patient with IAI. These data are essential for pathogendirected therapy $[42,197,199,200]$, and may provide the basis for de-escalation of initially broad-spectrum antimicrobial therapy [4,6,196-198].

12.1a. We recommend using measures of ongoing or progressive systemic inflammation or organ system dysfunction to identify patients with likely source control failure (Grade 1-B).

12.1b. We suggest that patients be assessed for source control failure if there is progressive organ dysfunction within the first 24-48 hours after source control, if there is no clinical improvement in organ dysfunction 48 hours or more after source control, or if there are persistent signs of inflammation 5-7 days after source control (Grade 2-C).

12.1c. We suggest that patients who clinically deteriorate or fail to improve within 48-72 hours of the initial procedure be considered for abdominal exploration (Grade 2-C). We recommend that patients suspected of treatment failure after 48-72 hours of the initial source control procedure undergo CT scanning, with percutaneous aspiration or drainage of any potentially infected fluid collections (Grade 1-B).

$12.1 d$. We recommend utilization of the least invasive approach that will either achieve definitive source control or will sufficiently control the infection such that there is resolution of the inflammatory response and organ dysfunction (Grade 1-B).

12.1e. We suggest that further source control be undertaken within 24 hours when failure of source control is identified (Grade 2-C). We suggest that patients with physiologic instability or progressive organ dysfunction should undergo further source control as soon as feasible (Grade 2-B).

12.1f. We recommend obtaining peritoneal cultures routinely in patients with source control failure, such that pathogen-directed antimicrobial therapy can be utilized (Grade 1-C).

\section{B. Antimicrobial therapy for treatment failure}

Q 12.2a. How should antimicrobial therapy be approached in patients with IAI who have treatment failure?

Q 12.2b. How should antimicrobial therapy be approached in patients with IAI who have clinical evidence of treatment failure but have negative imaging studies for persistent or recurrent IAI?

Q 12.2c. How should antimicrobial therapy be approached in patients with IAI who have clinical evidence of treatment failure and imaging studies showing inflammation but no discrete intra-abdominal source of infection amenable to source control?

Q 12.2d. How should antimicrobial therapy be approached in patients with IAI who have clinical evidence of treatment failure and imaging studies showing persistent or recurrent IAI, but in whom adequate source control cannot be achieved?

Early treatment failure in patients with IAI, occurring within 48 hours of source control, is typically the result of an unsuccessful initial intervention and not to a failure of anti- microbial therapy. The limited amount of anti-infective agents these patients have received is unlikely to have exerted much selection pressure on the pathogens that predominated at the time of the index procedure. In contrast, patients with late treatment failure, occurring after 48 hours, are likely to have a somewhat more resistant microbial flora because of longer-duration antimicrobial therapy. The differences between the micro-organisms associated with CA-IAI and HAIAI attest to this selection pressure [15,22-28,32,33]. The consensus of the task force is that continuation of previous antimicrobial therapy is appropriate in most patients with IAI who experience early treatment failure. Broader-spectrum empiric antimicrobial therapy, however, tailored to the expected risk of resistant pathogens, should be provided to patients with late treatment failure; if feasible, a switch in the class of antibiotic agents being administered should be considered.

Although successful management of IAI is usually heralded by resolution of fever, leukocytosis, and gastrointestinal dysfunction, these signs may persist in some patients, even in the absence of ongoing infection [653,654]. Patients who have persistent signs of inflammation should undergo diagnostic investigations to determine whether there is recurrent or persistent IAI, or an alternate source of infection. If diagnostic studies have negative results, further antimicrobial therapy for IAI is not generally warranted. Retrospective analyses have indicated that use of prolonged antibiotic courses, even in patients with ongoing fever or leukocytosis, does not provide any benefit [618,621,622].

Prospective studies using procalcitonin measurements to determine length of antimicrobial therapy also provided indirect evidence that treatment could be safely discontinued, even if not all signs of inflammation had resolved $[655,656]$. Moreover, the recent STOP-IT trial found that patients with IAI randomized to antimicrobial therapy continued two days beyond resolution of fever, leukocytosis, and ileus fared no better than patients randomized to having antibiotic agents stopped at four days, whether or not these signs were still present; this finding applied even to patients with higher APACHE II scores [82]. Taken together, these data support the tasks force's suggestion that antimicrobial therapy be discontinued in patients with IAI who have some clinical signs of treatment failure but whose imaging studies show no clear infectious source.

Imaging studies may be equivocal in some patients who have ongoing fever, leukocytosis, or ileus after an initial course of antimicrobial therapy for IAI. These studies may not identify a discrete source of infection, but may show areas of ongoing inflammation or small fluid collections within the abdomen, which cannot be accessed percutaneously. The importance of these findings is obscure. The task force did not identify any substantiative body of literature that addresses therapeutic approaches for such patients. Antimicrobial therapy alone, however, has been used for treating patients with some localized inflammatory processes because of IAI, such as a peri-appendiceal phlegmon or a small intraabdominal abscess related to diverticular or appendiceal disease [131-135,140-142]. Based on these considerations, the task force suggests that selected patients with clinical signs of treatment failure whose diagnostic studies show ongoing inflammation may receive a trial course of repeat antimicrobial therapy, preferably using agents distinct from those administered initially. Once this brief course of 
antimicrobial therapy is completed, however, the patient should not be given further anti-infective agents unless there is clear evidence of clinical deterioration or an imaging study documents progression of the inflammatory process.

Further source control should be the first consideration for treating patients with recurrent or persistent IAI identified by imaging studies. Further intervention may not be feasible, however, if the risk of surgical re-exploration is excessive and less invasive techniques cannot address the source of the infection. A decision made by the patient or a surrogate may also preclude further attempts at source control. The only option for these patients, then, is further antimicrobial therapy. There are few data regarding optimal anti-infective treatment of these patients. The task force suggested approach is based, in part, on current management practices for patients with infected pancreatitis. If at all possible, antimicrobial therapy should be guided by microbiologic data; potentially, cultures can be obtained by diagnostic techniques such as a fine needle aspiration when definitive source control is not an option $[657,658]$. Patients should be followed clinically and undergo repeat imaging studies while on antimicrobial therapy. If the patient's clinical examination improves substantially or if imaging studies reveal that the infection has abated, antimicrobial therapy can be discontinued. If subsequent imaging studies or a change in the patient's condition make source control a viable option, a procedure should be attempted. Periodic cessation of antimicrobial therapy should be attempted at intervals, even in the setting of persistent systemic inflammation, because indefinite treatment with antimicrobial agents will likely lead to development of resistant organisms. Nonetheless, antimicrobial therapy should not be discontinued in certain patients with IAI, particularly critically ill patients with uncontrolled tertiary peritonitis or worsening sepsis with organ failure $[8,629]$.

12.2a. We suggest that patients with IAI who have early treatment failure and undergo repeat source control within 48 hours of the initial source control intervention do not need to have antimicrobial therapy changed (Grade 2-C). We suggest that patients with IAI who have late treatment failure should have antimicrobial therapy changed to agents appropriate for HA-IAI; iffeasible, the class of antibiotic being provided should be switched (Grade 2-C).

12.2b. We suggest that patients with IAI who have clinical evidence of treatment failure but negative imaging studies for recurrent or persistent IAI have antimicrobial therapy discontinued (Grade 2-B).

12.2c. We suggest that patients with IAI who have clinical evidence of treatment failure and imaging studies showing ongoing intra-abdominal inflammation undergo a trial of additional antimicrobial agents appropriate for HA-IAI, using a different class of agents if feasible; if there is no clinical response to this antimicrobial trial within a few days, antimicrobial therapy should be discontinued, and only reinstated if there is evidence of clinical deterioration (Grade 2-C).

12.2d. We suggest that patients with IAI who have clinical evidence of treatment failure and imaging studies showing recurrent or persistent IAI, but in whom further source control cannot be achieved, have antimicrobial therapy continued; antimicrobial therapy should be discontinued when clinical signs of systemic inflammation or organ dysfunction abate
(Grade 2-C). We suggest that these patients be monitored for resistant pathogens, with antimicrobial therapy adjusted as necessary to treat such pathogens (Grade 2-C).

\section{Treatment of pediatric IAI}

$\mathrm{Q}$ 13.1a. What are the preferred agents for initial empiric antimicrobial therapy of lower-risk pediatric patients older than one month (45 wks post-conceptional age) with CA- IAI?

Q 13.1b. What are the preferred agents for initial empiric antimicrobial therapy of higher-risk pediatric patients older than one month (45 wks post-conceptional age) with CA-IAI and those with HA-IAI?

Q 13.2a. What should the duration of antimicrobial therapy be for pediatric patients older than one month (45 wks post-conceptional age) with IAI?

Q 13.2b. Should supplemental oral antimicrobial therapy be provided to pediatric patients with IAI after they have received IV therapy?

Q 13.2c. Should outpatient IV antimicrobial therapy be provided to pediatric patients with IAI who can be discharged from the hospital?

$Q$ 13.3. How should treatment failure be managed in pediatric patients with IAI?

Q 13.4a. How should pediatric patients less than one month old (45 wks post-conceptional age) with necrotizing enterocolitis (NEC) or intestinal perforation be treated?

Q 13.4b. What are the preferred antimicrobial agents for initial empiric therapy of pediatric patients less than one month old (45 wks post-conceptional age) with IAI?

Q 13.4c. What should be the duration of antimicrobial therapy for pediatric patients with IAI less than one month old (45 wks post-conceptional age)?

$\mathrm{Q}$ 13.5. What dosages of antimicrobial agents should be used for treatment of pediatric patients with IAI?

The most common IAI encountered in children outside of the neonatal age group is complicated appendicitis; IAI from other sources is observed much less frequently [659]. As with adults, empiric antimicrobial therapy directed against gramnegative Enterobacteriaceae and anaerobic bacteria is appropriate for children with perforated appendicitis and other types of IAI [660,661]. Previously, a combination of ampicillin, an aminoglycoside, and an anti-anaerobic agent was considered the gold standard in treating pediatric patients with IAI. Numerous studies, however, have demonstrated efficacy of other antimicrobial regimens for managing IAI in these patients [328,661]. RCTs published before 2000 are included in the previous evidence review [3], and newer studies are summarized in Supplementary Table V; see online supplementary material at www.liebertpub.com/overview/ surgical-infections/53/. These RCTs support use of ticarcillinclavulanic acid [220,662,663], piperacillin-tazobactam [225,327], ertapenem [205,214,220], imipenem-cilastatin [664,665], meropenem [290,666], cefoperazone-sulbactam [203], and combinations of cefotaxime [225,667], ceftriaxone [204,212,319,668], or ceftazidime [210] with metronidazole or clindamycin. In addition, cefuroxime has been approved for use in pediatric patients [523], and cefepime [669] and aztreonam [670] have efficacy for managing 
serious pediatric infections, although studies have not specifically addressed their use in pediatric IAI.

Age-based restrictions to use of fluoroquinolone antibiotic agents in pediatric patients were advocated in the past. Investigational studies documented cartilage injury in weightbearing joints of juvenile canines [671,672], although the importance of these findings was uncertain. A systematic review of the literature suggested that any musculoskeletal adverse events developing in pediatric patients were reversible [303]. The American Association of Pediatrics Committee on Infectious Diseases has recommended the use of fluoroquinolones in certain clinical scenarios, and many centers have adopted this practice [304]. The task force has therefore suggested that ciprofloxacin with metronidazole can be used as an alternative regimen for treating pediatric patients with IAI when other regimens are not suitable, particularly in patients who have major reactions to $\beta$-lactam antibiotic agents.

As with adult patients, data demonstrating superiority of any specific antimicrobial regimen for the management of IAI in pediatric patients are lacking [328]. Thus, the task force recommendations regarding selection of specific antimicrobial agents are based on factors such as toxicity, convenience, and cost, with particular emphasis placed on considerations of antimicrobial stewardship. For CA-IAI in lower-risk pediatric patients older than one month, monotherapy with ertapenem or combination therapy with cefotaxime or ceftriaxone plus metronidazole are recommended. An alternative regimen, not nearly as well investigated, is cefuroxime plus metronidazole. The use of ciprofloxacin plus metronidazole can be considered in patients with severe $\beta$-lactam reactions (Table 14). The use of aminoglycosidebased regimens is discouraged, because of evidence of decreased efficacy $[207,208]$ as well as the toxicity associated with these agents.

Although recommended by some, the task force does not advocate use of routine anti-pseudomonal coverage for

TABle 14. ReCOMMENDED EMPIRIC ANTIMICROBIAL Regimens for Pediatric Patients Older Than One Month With COMMUnity-ACQuired INTRA-ABDOMINAL INFECTION*

\begin{tabular}{|c|c|}
\hline Lower-risk patients & Higher-risk patients \\
\hline \multicolumn{2}{|l|}{ Preferred regimens } \\
\hline $\begin{array}{l}\text { Cefotaxime or ceftriaxone } \\
\text { plus metronidazole }\end{array}$ & Piperacillin-tazobactam \\
\hline Ertapenem & $\begin{array}{l}\text { Imipenem-cilastatin } \\
\text { Meropenem }\end{array}$ \\
\hline Alternative regimens & \\
\hline $\begin{array}{l}\text { Cefuroxime plus } \\
\text { metronidazole }\end{array}$ & $\begin{array}{l}\text { Ceftazidime or cefepime } \\
\text { plus metronidazole } \\
\text { Aztreonam plus metronidazole } \\
\text { plus vancomycin }\end{array}$ \\
\hline $\begin{array}{l}\text { Optional regimens } \\
\text { Ciprofloxacin or } \\
\text { levofloxacin plus } \\
\text { metronidazole }\end{array}$ & \\
\hline
\end{tabular}

\footnotetext{
*More than 45 weeks post-conceptual age.

a Use of an agent effective against Enterococcus spp. is suggested in patients with severe sepsis/septic shock who receive a cephalosporin-based regimen.
}

lower-risk pediatric patients with CA-IAI. Pseudomonas spp. has been isolated with variable frequency from peritoneal cultures of pediatric patients with complicated appendicitis. One series identified $P$. aeruginosa in $31 \%$ of such cultures [673], but the incidence was substantially lower in other series $[20,393,674]$. In addition, RCTs have not demonstrated improved outcomes in pediatric patients receiving regimens with anti-pseudomonal coverage compared with patients receiving regimens without that coverage $[203,205,220,226,675]$.

There is minimal evidence with regard to antimicrobial therapy of higher-risk children with CA-IAI or those with HA-IAI. The task force suggests that recommendations for higher-risk pediatric patients parallel those for higher-risk adult patients. Broader-spectrum empiric therapy should be provided initially in pediatric patients with severe sepsis or septic shock, because of the potential for inadequate therapy if a more resistant organism, such as Pseudomonas spp., is encountered. Use of piperacillin-tazobactam, imipenemcilastatin, or meropenem is suggested in these patients. Alternative regimens, not as well studied in pediatric patients, include ceftazidime or cefepime plus metronidazole. Additional coverage of Enterococcus spp., MRSA, MDR gramnegative bacilli, or yeast should be considered in pediatric patients with HA-IAI, if they have risk factors for infection with those resistant micro-organisms.

Previously, pediatric patients were frequently prescribed seven or more days of IV antibiotic therapy after source control for perforated appendicitis or other types of IAI $[387,673]$. A recent RCT indicated that five days of IV antimicrobial therapy was sufficient for these patients (Supplementary Table W; see online supplementary material at www.liebertpub.com/overview/surgical-infections/53/) [319]. Other authorities have advocated earlier discontinuation of antimicrobial therapy in pediatric patients with IAI, either based on a fixed duration of therapy or resolution of clinical signs of infection [328,661,676,677]. Although RCTs have found that a 3-4 day course of antimicrobial therapy is adequate in adult patients $[82,214,623]$, the task force recommends a five-day total duration of antimicrobial therapy in pediatric patients with IAI, because of the lack of evidence on shorter-duration therapy in those patients.

There is increasing acceptance of the option to transition pediatric patients to oral antibiotic agents once they are afebrile and tolerating a regular diet. Two RCTs in pediatric patients found a switch to oral amoxicillin-clavulanic acid equivalent to use of IV therapy only (Supplementary Table $\mathrm{O}$; see online supplementary material at www.liebertpub. com/overview/surgical-infections/53/) [319,320]. Other retrospective studies suggested this agent or a combination of trimethoprim-sulfamethoxazole plus metronidazole were adequate to complete a course of antimicrobial therapy [387,388].

There are no data, however, indicating that administering oral antibiotic agents after completing a full course of IV antibiotic agents provides any additional benefit $[319,677]$. Thus, oral antibiotic agents should be used only to complete a recommended five-day course of antimicrobial therapy and not to extend therapy beyond that $[328,678]$. Similarly, because there are no data suggesting a benefit of additional IV antibiotic agents beyond five days $[319,661,678]$, the use of outpatient IV antimicrobial therapy would generally be 
unnecessary for most pediatric patients with IAI. Outpatient IV therapy to complete a five-day course could be considered in the infrequent patient with IAI because of a resistant organism for which no oral agent was suitable. Overall, in the absence of treatment failure, the task force recommends against use of additional oral or IV antibiotic agents beyond five days in pediatric patients with IAI.

In pediatric IAI, complications developing after management of perforated appendicitis are the most common examples of treatment failure. One large observational study documented a $13 \%$ risk of an intra-abdominal abscess in pediatric patients with perforated appendicitis [677]. Percutaneous drainage of an abscess provides adequate source control, although small abscesses may be treatable with antibiotic agents alone $[679,680]$. Further antimicrobial therapy should follow the principles outlined previously for adult patients with treatment failure. There is no evidence that prolonged antimicrobial therapy benefits pediatric patients with a post-operative abscess. In the presence of adequate source control, the task force suggests no more than seven days of antimicrobial therapy for treatment failure in children. These patients may be converted to oral therapy to complete their course of therapy [319], as is done for children with primary IAI. In the absence of adequate source control, however, such as in patients with continued soilage of the abdomen from an appendiceal stump leak, more prolonged antimicrobial therapy may be warranted, with the proviso that definitive source control should be attempted whenever feasible.

IAI in neonatal patients develops most commonly as a result of NEC or spontaneous intestinal perforation; other etiologies, such as post-operative IAI, are also encountered [659]. Source control in premature infants is generally considered imperative. Both laparotomy with resection and peritoneal drainage have been used, however, to treat patients with spontaneous and NEC-induced intestinal perforations [681]. Two RCTs (Supplementary Table X; see online supplementary material at www.liebertpub.com/overview/surgical-infections/53/) comparing these two approaches showed no differences in mortality; however, considerably different rates of salvage laparotomy were observed in patients treated with initial peritoneal drainage in the two studies $[682,683]$.

There is little prospective evidence regarding optimal antimicrobial therapy for infants with IAI. A few RCTs comparing antibiotic regimens for patients with NEC have been published, but these have evaluated primarily treatment of patients with clinical evidence of NEC without overt perforation; the studies have not addressed directly antimicrobial therapy of patients with established IAI due to intestinal perforation [684]. Thus, recommendations for antimicrobial therapy are based primarily on indirect evidence from studies of NEC and expert opinion [685-687]. In general, the task force believes that antimicrobial therapy in neonatal patients with IAI should conform to the general principles outlined for treatment of HA-IAI. Multi-drug combinations have frequently been used in this high-risk population [688], but monotherapy providing equivalent broad-spectrum gramnegative coverage is acceptable. Selective addition of agents with activity against enterococci, MRSA, and yeast should be considered in patients with risk factors for these resistant organisms, most commonly previous broad-spectrum antibiotic use. Vancomycin has been used for infections suspected to be from MRSA or ampicillin-resistant enterococci.
Table 15. Summary of Empiric Antimicrobial Therapy for Pediatric Patients Less than One MONTH OLD WITH INTRA-ABDOMINAL INFECTION*

General recommendation

Ampicillin, gentamicin, plus metronidazole Ampicillin, cefotaxime, plus metronidazole Meropenem

Additional agents

\begin{tabular}{ll}
\hline Potential pathogen & \multicolumn{1}{c}{ Suggestion } \\
\hline Enterococcus spp. & $\begin{array}{l}\text { Use of ampicillin if E. faecalis } \\
\text { is suspected, or use of vancomycin } \\
\text { instead of ampicillin if a penicillin- } \\
\text { resistant Enterococcus spp. }\end{array}$ \\
is suspected & Use of vancomycin if MRSA suspected \\
Candida spp. & Use of amphotericin B or fluconazole
\end{tabular}

*Less than 45 weeks post-conceptual age.

MRSA = methicillin-resistant Staphylococcus aureus .

Amphotericin B and fluconazole have been prescribed for fungal peritonitis. Once source control has been achieved, the task force suggests that antimicrobial therapy be continued for 7-10 days. These consensus recommendations for antimicrobial treatment of neonatal patients up to one month of age with IAI are shown in Table 15.

Dosing recommendations for pediatric patients both younger and older than one month are listed in Table 16. Where possible, these dosing recommendations are derived from RCTs (Supplementary Table X; see online supplementary material at www.liebertpub.com/overview/surgicalinfections/53/) performed in pediatric patients. Other sources include the prescribing information for specific antimicrobial agents approved by the FDA [523] and a standard pharmacology text [524]. As with adult patients, standard dosing is appropriate for nearly all lower-risk children with CA-IAI. Higher doses, however, may be reasonable in critically ill pediatric patients, who may have an increased volume of distribution and/or accelerated clearance of antimicrobial agents. Optimal dosing of many antimicrobial agents in the neonatal age group is extrapolated from data on older children, because pharmacokinetic studies on these younger patients are frequently lacking.

13.1a. We recommend ertapenem or a combination of cefotaxime or ceftriaxone plus metronidazole for empiric antimicrobial therapy of CA-IAI in lower-risk pediatric patients older than one month (45 wks post-conceptional age) (Grade 1-A). We suggest cefuroxime plus metronidazole as an alternative regimen for empiric treatment of these pediatric patients (Grade 2-B). We suggest ciprofloxacin or levofloxacin plus metronidazole as acceptable regimens for empiric treatment of selected pediatric patients with IAI if other agents cannot be used, particularly for those pediatric patients with life-threatening $\beta$-lactam reactions (Grade 2$B$ ). We suggest cefoperazone-sulbactam, where it is available, as an acceptable option for empiric therapy of CA-IAI in lower-risk pediatric patients (Grade 2-B).

13.1b. We suggest piperacillin-tazobactam, imipenemcilastatin, or meropenem for empiric antimicrobial therapy of CA-IAI in higher-risk pediatric patients older than one month (45 wks post-conceptional age) (Grade 2-A). We also 
Table 16. Dosages of Antimicrobial Agents for Pediatric Patients

\begin{tabular}{|c|c|c|}
\hline \multirow[b]{2}{*}{ Agent } & \multicolumn{2}{|l|}{ Age } \\
\hline & $\begin{array}{l}\text { Children older than } \\
\text { one month of age }\end{array}$ & $\begin{array}{l}\text { Term infants to } \\
\text { one month of age }\end{array}$ \\
\hline \multicolumn{3}{|l|}{ Aminoglycosides } \\
\hline Amikacin & $5-7.5 \mathrm{mg} / \mathrm{kg}$ IV q8h & \multirow{3}{*}{ 4-5 mg/kg IV q24-48h } \\
\hline Gentamicin & $2.5 \mathrm{mg} / \mathrm{kg}$ IV q8h & \\
\hline Tobramycin & $2-2.5 \mathrm{mg} / \mathrm{kg}$ IV q $8 \mathrm{~h}$ & \\
\hline \multicolumn{3}{|l|}{ Aminopenicillin } \\
\hline Ampicillin & $100-400 \mathrm{mg} / \mathrm{kg} / \mathrm{d} \mathrm{IV}$ in divided doses q6h & $50 \mathrm{mg} / \mathrm{kg}$ IV q6-12h \\
\hline \multicolumn{3}{|c|}{$\beta$-lactamase/ $\beta$-lactamase inhibitor combinations } \\
\hline Ampicillin-sulbactam & $\begin{array}{l}100-200 \mathrm{mg} \text { ampicillin/kg/d IV } \\
\text { in divided doses } \mathrm{q} 6 \mathrm{~h}\end{array}$ & \multirow{11}{*}{$50 \mathrm{mg} / \mathrm{kg}$ IV q8-12h } \\
\hline $\begin{array}{l}\text { Ticarcillin-clavulanate } \\
\text { (Not currently available) }\end{array}$ & $200-300 \mathrm{mg} / \mathrm{kg} / \mathrm{d} \mathrm{IV}$ in divided doses q4-6h & \\
\hline Piperacillin-tazobactam & $240-300 \mathrm{mg} / \mathrm{kg} / \mathrm{d}$ IV in divided doses $\mathrm{q} 6-8 \mathrm{~h}$ & \\
\hline \multicolumn{3}{|l|}{ Cephalosporins } \\
\hline Cefoxitin & $80-160 \mathrm{mg} / \mathrm{kg} / \mathrm{d}$ IV in divided doses q6-8h & \\
\hline Cefotetan & $20-40 \mathrm{mg} / \mathrm{kg}$ IV q12h & \\
\hline Cefazolin & $25-50 \mathrm{mg} / \mathrm{kg}$ in $3-4$ divided doses $\mathrm{q} 6-8 \mathrm{~h}$ & \\
\hline Cefuroxime & $75-200 \mathrm{mg} / \mathrm{kg} / \mathrm{d}$ IV in divided doses $\mathrm{q} 6-8 \mathrm{~h}$ & \\
\hline Cefotaxime & $\begin{array}{l}50-180 \mathrm{mg} / \mathrm{kg} / \mathrm{d} \text { IV in divided doses } \mathrm{q} 4-6 \mathrm{~h} \\
\text { for patients }<50 \mathrm{~kg} \text {; adult dosages } \\
\text { for patients }>50 \mathrm{~kg}\end{array}$ & \\
\hline Ceftriaxone & $50-100 \mathrm{mg} / \mathrm{kg} / \mathrm{d}$ IV in $1-2$ divided doses & \\
\hline Ceftazidime & $30-50$ mg/kg IV q8h & \\
\hline Cefepime & $\begin{array}{l}50 \mathrm{mg} / \mathrm{kg} \text { IV } \mathrm{q} 12 \mathrm{~h}(\leq 16 \mathrm{y} \text { and } \leq 40 \mathrm{~kg}) \\
\text { adult dosages for }>16 \text { y or }>40 \mathrm{~kg})\end{array}$ & \\
\hline \multicolumn{3}{|c|}{ Cephalosporin/ $\beta$-lactamase inhibitor combination } \\
\hline \multicolumn{3}{|l|}{ Cefoperazone-sulbactam: } \\
\hline $2: 1$ ratio & 40-80 mg/kg/a divided qo-1 $2 \mathrm{n}$ & \\
\hline \multirow{2}{*}{\multicolumn{3}{|c|}{ Monobactam }} \\
\hline & & \\
\hline Aztreonam & $30 \mathrm{mg} / \mathrm{kg}$ IV q6-8h (>9 mo) & \\
\hline \multicolumn{3}{|l|}{ Carbapenems } \\
\hline Ertapenem & $15 \mathrm{mg} / \mathrm{kg}$ IV q12h $(\leq 12 \mathrm{y}) ;$ adult dosages for $>12 \mathrm{y}$ & \\
\hline Imipenem/cilastatin & $15-25 \mathrm{mg} / \mathrm{kg} \mathrm{IV} \mathrm{q6h}$ & \\
\hline Meropenem & $30-120 \mathrm{mg} / \mathrm{kg} / \mathrm{d}$ IV in divided doses $\mathrm{q} 8 \mathrm{~h}$ & $20 \mathrm{mg} / \mathrm{kg}$ IV q8-12h \\
\hline \multicolumn{3}{|l|}{ Fluoroquinolones } \\
\hline Ciprofloxacin & $20-30 \mathrm{mg} / \mathrm{kg} / \mathrm{d} \mathrm{IV}$ in divided doses q12h & \\
\hline Levofloxacin & $\begin{array}{l}10 \mathrm{mg} / \mathrm{kg} \text { IV q12h }(6 \mathrm{mo} \text { to } 5 \mathrm{y}) \\
10 \mathrm{mg} / \mathrm{kg} \mathrm{IV} \mathrm{q} 24 \mathrm{~h}(\geq 5 \mathrm{y})\end{array}$ & \\
\hline \multicolumn{3}{|l|}{ Anti-anaerobic agents } \\
\hline Clindamycin & $20-40 \mathrm{mg} / \mathrm{kg} / \mathrm{d}$ IV in 3-4 divided doses & $5-7.5 \mathrm{mg} / \mathrm{kg}$ IV q8-12h \\
\hline Metronidazole & $22.5-40 \mathrm{mg} / \mathrm{kg} / \mathrm{d} \mathrm{IV}$ in divided doses $\mathrm{q} 8 \mathrm{~h}$ & $15 \mathrm{mg} / \mathrm{kg}$ IV q12-24h \\
\hline \multicolumn{3}{|c|}{ Agents with activity against resistant gram-positive organisms } \\
\hline Vancomycin & $10 \mathrm{mg} / \mathrm{kg}$ IV q6h & $10-15 \mathrm{mg} / \mathrm{kg}$ IV g8-12h \\
\hline Teicoplanin & $\begin{array}{l}10 \mathrm{mg} / \mathrm{kg} \text { IV q8} 8-12 \mathrm{~h} \times 3 \text { doses, } \\
\text { then } 6-10 \mathrm{mg} / \mathrm{kg} \text { IV q24h }\end{array}$ & \\
\hline Linezolid & $10 \mathrm{mg} / \mathrm{kg}$ IV q8h $(<12 \mathrm{y})$ & \\
\hline \multicolumn{3}{|l|}{ Antifungal agents } \\
\hline Amphotericin B deoxycholate & $1-1.5 \mathrm{mg} / \mathrm{kg}$ IV q24h & $\begin{array}{l}1 \mathrm{mg} / \mathrm{kg} / \mathrm{d} \text { IV or } \\
1.5 \mathrm{mg} / \mathrm{kg} \text { IV } \mathrm{q} 48 \mathrm{~h}\end{array}$ \\
\hline $\begin{array}{l}\text { Amphotericin B lipid complex, } \\
\text { amphotericin B, colloidal } \\
\text { dispersion, liposomal } \\
\text { amphotericin B }\end{array}$ & $5 \mathrm{mg} / \mathrm{kg}$ IV q24h & $5 \mathrm{mg} / \mathrm{kg}$ IV q24h \\
\hline Caspofungin & $70 \mathrm{mg} / \mathrm{m}^{2} \mathrm{IV} \times 1$ dose, then $50 \mathrm{mg} / \mathrm{m}^{2} \mathrm{q} 24 \mathrm{~h}$ & \\
\hline Fluconazole & $3-12 \mathrm{mg} / \mathrm{kg}$ IV q24h & $3-12 \mathrm{mg} / \mathrm{kg}$ IV q24h \\
\hline
\end{tabular}

${ }^{a}$ Older or younger than 45 weeks post-conceptual age.

$\mathrm{IV}=$ intravenous. 
suggest these agents for empiric therapy of HA-IAI in pediatric patients (Grade 2-B). We suggest ceftazidime or cefepime plus metronidazole as alternative regimens for empiric treatment of CA-IAI or HA-IAI in these pediatric patients (Grade 2-B). We suggest aztreonam plus metronidazole plus vancomycin as an acceptable regimen for empiric treatment of selected pediatric patients if other agents cannot be used, particularly for pediatric patients with life-threatening $\beta$ lactam reactions (Grade 2-B). We suggest addition of ampicillin or vancomycin as empiric anti-enterococcal therapy of CA-IAI in higher-risk patients and those with HA-IAI if the patient is not being treated with piperacillin-tazobactam or imipenem-cilastatin (Grade 2-B).

13.2a. We recommend no more than five full days $(120 \mathrm{~h})$ of antimicrobial therapy of IAI in pediatric patients older than one month (45wks post-conceptional age) who have had adequate source control (Grade 1-A).

13.2b. We recommend against use of additional oral antimicrobial therapy for IAI in pediatric patients who have had adequate source control, unless given to complete a total antimicrobial course of five days (Grade 1-B).

13.2c. We recommend against use of additional outpatient IV antimicrobial therapy for IAI in pediatric patients who have had adequate source control, unless given to complete a total antimicrobial course of five days (Grade 1-B).

13.3. We recommend that pediatric patients with treatment failure be managed in an analogous fashion to adult patients with treatment failure (Grade 1-C). We suggest using the least invasive means of providing adequate source control and using a standard course of IV antibiotic agents; a change in antibiotic class may be considered in these patients (Grade 2-C). We recommend against use of IV antibiotic agents beyond seven days for children with perforated appendicitis who have a post-operative abscess (Grade 1-C).

13.4a. We recommend either laparotomy or peritoneal drainage as source control in addition to antimicrobial therapy for pediatric patients less than one month of age (45 wks post-conceptional age) with NEC or intestinal perforation (Grade 1-A).

13.4b. We suggest use of ampicillin, gentamicin, and metronidazole or clindamycin; ampicillin, cefotaxime, and metronidazole or clindamycin; or meropenem in pediatric patients less than one month of age (45 wks post-conceptional age) with IAI. Vancomycin may be used instead of ampicillin if there is suspected infection with penicillin-resistant Enterococcus spp. or MRSA. Fluconazole or amphotericin B can be added if there is a suspected infection with Candida spp. (Grade 2-C).

13.4c. We suggest a 7-10 day course of antimicrobial therapy for pediatric patients less than one month of age (45 wks post-conceptional age), particularly for those with NEC (Grade 2-C).

13.5. We recommend use of standard pediatric dosages for various antimicrobial agents for lower-risk pediatric patients with CA-IAI (Grade 1-B). We suggest use of higher pediatric dosages, where applicable, for higher-risk patients with CAIAI and those with HA-IAI (Grade 2-C).

\section{Recommendations for Future Investigations}

The development of this revised guideline for the management of IAI has uncovered numerous problems for which data are inadequate to allow firm conclusions. Key questions remain both with respect to optimal source control and to utilization of antimicrobial therapy in patients with IAI.

Many recommendations in these guidelines are stratified based on the perceived risk that a given patient will have an adverse outcome. An adverse outcome has been defined variably in the literature and may refer to treatment failure or to death. Treatment failure, in turn, has been variably defined, ranging from persistent signs of infection to a need for further invasive interventions for source control. At present, a simple tool for calculating risk of either treatment failure or death does not yet exist.

One goal of future investigations would be to construct and validate simple, reliable tools for assessing risk of source control failure and death, such that they can be utilized for planning source control and antimicrobial therapy at the point of care. Relatively simple, revised criteria for identifying patients with sepsis or septic shock have been proposed by the Society of Critical Care Medicine and the European Society of Intensive Care Medicine [80], which may simplify the identification of patients with IAI who have sepsis or septic shock. For purposes of treating patients with IAI, however, additional measures are needed, because not all higher-risk patients with IAI meet these revised criteria for sepsis or septic shock.

Options for source control in patients with IAI continue to evolve. Relatively few new procedures have been evaluated prospectively. There is little doubt that less invasive procedures can provide adequate source control for patients with IAI, and that extensive procedures performed to maximize source control may produce greater morbidity and death than less extensive procedures. Nonetheless, less invasive source control measures may not lead to improved patient outcomes. New procedures should be adequately investigated and not adopted prematurely for patient care. An example is the current controversy over the use of less invasive procedures for managing Hinchey Stage III and IV complicated diverticulitis [154-159].

Given the wide variety of potential source control interventions that could be tested, it is unrealistic to expect RCTs can be used to analyze even a small fraction of these approaches. Non-randomized cohort studies, however, can provide valuable information, particularly if care is taken to ensure that control patients are carefully matched with regard to prognostic factors. The use of large, multi-institutional databases, such as that obtained from the Complicated IntraAbdominal Infection Observational Study, can facilitate these observations and make them more generalizable [26,438]. The SIS and similar organizations should consider developing similar databases to analyze best practices with regard to source control.

The principles of antimicrobial therapy for patients with IAI are well established. The increasing prevalence of ESBLproducing and fluoroquinolone-resistant strains of $E$. coli and other gram-negative organisms in patients with IAI, however, could have a major impact on antimicrobial selection [28]. These resistance concerns extend to patients with CA-IAI as well as those with HA-IAI. As of yet, it is unclear whether the outcomes of lower-risk patients with CA-IAI will significantly worsen because of these resistant organisms. Although data from a number of RCTs suggest that narrower-spectrum empiric antimicrobial agents are still effective in these patients [204,205,220,225,228-230,252,255,294-296], larger-scale, contemporary investigations are needed to determine the impact of this increasing resistance on outcomes in patients with IAI. 
Future investigations should focus on preventing the number of deaths in higher-risk patients, both those with CAIAI and those with HA-IAI. In general, the adequacy of initial empiric antimicrobial therapy has been thought to have a major impact on the deaths and morbidity of higher-risk patients [43,54,58,71,187,193-195]. This guideline recommends use of empiric anti-enterococcal and antifungal therapy in many of these patients, and also recommends use of empiric agents that have broader-spectrum activity against gram-negative pathogens. The increase in resistant microorganisms makes the selection of appropriate empiric antimicrobial agents for these patients more difficult, but overuse of broad-spectrum agents may lead to even greater resistance problems. The hypothesis that outcomes in severely ill patients with IAI are improved by using broader-spectrum empiric antimicrobial effective against all the expected pathogens should be evaluated prospectively through a methodologically sound trial to determine whether empiric use of broad-spectrum antimicrobial agents, as well as antienterococcal, anti-MRSA, and antifungal agents, is actually necessary. To better examine this hypothesis, RCTs of new antimicrobial agents with activity against resistant microorganisms should include adequate numbers of severely ill patients likely to harbor such resistant pathogens.

There are relatively few new anti-infective agents on the horizon that are likely to overcome these resistance problems, and none is likely to be a panacea. Among newer agents undergoing investigation, published phase II data are available only for the use of eravacycline and imipenem-cilastatin with relabactam in patients with IAI $[689,690]$. Most patients with IAI will therefore continue to be treated with conventional antimicrobial agents. The application of the principles of antimicrobial stewardship will be of utmost importance in conserving these antimicrobial resources. Stratification of empiric antimicrobial therapy according to patient risk and de-escalation of broad-spectrum therapy once culture results have been obtained have been emphasized in this guideline as approaches to antimicrobial stewardship. There is a strong potential, however, that newer diagnostic microbiologic modalities, which provide information on the presence of important pathogens within hours rather than days, will allow earlier deployment of pathogen-directed antimicrobial therapy. As these methodologies mature, prospective studies should be undertaken to document their potential benefits not only in improving outcomes in patients with IAI, but also in decreasing use of broad-spectrum empiric anti-infective therapy.

Other means of promoting antimicrobial stewardship have also been endorsed with these recommendations. Decreasing the duration of antimicrobial therapy to the minimum necessary to achieve a maximal clinical benefit is another fundamental principle of antimicrobial stewardship. The SIS STOP-IT trial has provided valuable data indicating that duration of therapy can be safely limited in adult patients with IAI [82]. Limiting duration of antimicrobial therapy even further, for example, to two days in patients with perforated appendicitis, has been proposed [617]; this hypothesis could be evaluated rigorously in future studies.

Optimizing dosing of antimicrobial agents provides another means of conserving antimicrobial resources. Altered pharmacokinetic parameters are common in critically ill patients, including those with IAI, with resultant underdosing and overdosing of anti-infective agents. The use of extended infusions to optimize pharmacodynamics of these agents is an attractive approach, but has not yet shown to have clear clinical benefits in patients with IAI. Optimization of antimicrobial dosing for patients at the extremes of age, obese patients, and those with renal or hepatic impairment has not been investigated sufficiently; such studies should be considered, because these are common risk factors in the most severely ill patients with IAI.

Patients with treatment failure are at substantially increased risk for morbidity and death. The ideal means of providing source control in these patients has not been fully elucidated. There are clearly trade-offs between less aggressive approaches, which may lead to incomplete source control, and more invasive approaches, which may lead to excess procedural morbidity and death. The development of more standardized approaches to source control in these patients could potentially improve the outcomes of these severely ill patients.

There are also numerous questions related to the treatment of pediatric patients with IAI. The use of less invasive procedures for source control in pediatric patients with IAI is becoming widespread. Careful evaluation as to the efficacy of these interventions should be ongoing. Although there is good evidence that non-aminoglycoside-based antimicrobial regimens provide equivalent if not superior results in most pediatric patients with IAI, aminoglycoside-based regimens continue to be widely used in neonatal patients. Studies of alternative regimens are needed to avoid the potential side effects of these agents. Another important question is whether or not antimicrobial therapy can be limited further in pediatric patients with IAI; extended duration therapy is still frequently used in these patients.

Although this guideline has focused on the management of patients with IAI, ongoing investigations into the pathophysiology of IAI should not be discounted. These investigations may provide an avenue toward novel ways of treating patients with these infections. The pioneering studies performed nearly 50 years ago on the importance of both aerobic gram-negative bacilli and anaerobic organisms in the pathophysiology of IAI led directly to the principles used today for providing antimicrobial therapy to these patients. Currently, some of the most exciting work relates to the role of the gastrointestinal microbiome as a contributor to both illness and health. With the advent of increasingly sophisticated tools to identify the wide variety of micro-organisms associated with gastrointestinal tract pathology, our understanding of the intricate relationship between the resident microbial population and the host will be greatly amplified. It would not be unexpected that antimicrobial therapy and potentially source control approaches for patients with IAI will be modified through the use of this knowledge. It is even possible that future therapy of IAI could involve use of probiotic agents to restore a healthy internal ecosystem within the gastrointestinal tract, rather than the antibiotic agents currently used.

\section{Acknowledgments}

The guideline was prepared by a Task Force of the Surgical Infection Society, including members of the Therapeutics and Guidelines Committee, and approved by the Council of the Surgical Infection Society as an official guideline developed by the organization. 
The task force would like to acknowledge the careful review, suggestions for changes, and editing of this document provided by Phil Barie, William Cheadle, and Don Fry, and the assistance of Lynn Hydo in coordinating task force activities.

\section{Author Disclosure Statement}

During the preparation of the guideline, JEM has received research support from AstraZeneca, Bayer HealthCare Pharmaceuticals, and Merck \& Co., and served as an advisory board member, consultant, or speaker for Allergan, Bayer Pharmaceuticals, and Merck \& Co. AKM has received research support from Cubist Pharmaceuticals, Inc., Fresenius Kabi, Bayer HealthCare Pharmaceuticals Inc., and Atox Bio Ltd., and served as a consultant or speaker to Dr. Reddy's Laboratory, Inc., Bayer HealthCare Pharmaceuticals, Inc., Atox Bio, Ltd., and Pfizer Inc. RGS has served as a consultant to Merck \& Co. and 3M Co. For the remaining authors, no competing financial interests exist.

\section{References}

1. Bohnen JM, Solomkin JS, Dellinger EP, et al. Guidelines for clinical care: Anti-infective agents for intra-abdominal infection. A Surgical Infection Society policy statement. Arch Surg 1992;127:83-89.

2. Mazuski JE, Sawyer RG, Nathens AB, et al. The Surgical Infection Society guidelines on antimicrobial therapy for intra-abdominal infections: An executive summary. Surg Infect (Larchmt) 2002;3:161-173.

3. Mazuski JE, Sawyer RG, Nathens AB, et al. The Surgical Infection Society guidelines on antimicrobial therapy for intra-abdominal infections: Evidence for the recommendations. Surg Infect (Larchmt) 2002;3:175-233.

4. Solomkin JS, Mazuski JE, Bradley JS, et al. Diagnosis and management of complicated intra-abdominal infection in adults and children: Guidelines by the Surgical Infection Society and the Infectious Diseases Society of America. Surg Infect (Larchmt) 2010;11:79-109.

5. Solomkin JS, Mazuski JE, Baron EJ, et al. Guidelines for the selection of anti-infective agents for complicated intraabdominal infections. Clin Infect Dis 2003;37:997-1005.

6. Dellinger RP, Levy MM, Rhodes A, et al. Surviving Sepsis Campaign: International guidelines for management of severe sepsis and septic shock: 2012. Crit Care Med 2013;41:580-637.

7. Guyatt GH, Oxman AD, Kunz R, et al. GRADE: What is "quality of evidence" and why is it important to clinicians? BMJ 2008;336:995-998.

8. Guyatt GH, Oxman AD, Kunz R, et al. Going from evidence to recommendations. BMJ 2008;336:1049-1051.

9. Atkins D, Best D, Briss PA, et al. Grading quality of evidence and strength of recommendations. BMJ 2004; 328:1490.

10. Bero L, Rennie D. The Cochrane Collaboration: Preparing, maintaining, and disseminating systematic reviews of the effects of health care. JAMA 1995;274:1935-1938.

11. Jadad AR, Moore RA, Carroll D, et al. Assessing the quality of reports of randomized clinical trials: Is blinding necessary? Control Clin Trials 1996;17:1-12.

12. Solomkin JS, Hemsell DL, Sweet R, et al. Evaluation of new anti-infective drugs for the treatment of intraabdominal infections. Infectious Diseases Society of America and the
Food and Drug Administration. Clin Infect Dis 1992; 15(Suppl 1):S33-S42.

13. Blot S, De Waele JJ. Critical issues in the clinical management of complicated intra-abdominal infections. Drugs 2005;65:1611-1620.

14. Calandra T, Cohen J, International Sepsis Forum Definition of Infection in the ICU Consensus Conference. The international sepsis forum consensus conference on definitions of infection in the intensive care unit. Crit Care Med 2005;33:1538-1548.

15. Chromik AM, Meiser A, Hölling J, et al. Identification of patients at risk for development of tertiary peritonitis on a surgical intensive care unit. J Gastrointest Surg 2009;13: 1358-1367.

16. Mishra SP, Tiwary SK, Mishra M, Gupta SK. An introduction of tertiary peritonitis. J Emerg Trauma Shock 2014; 7:121-123.

17. Seguin P, Laviolle B, Chanavaz C, et al. Factors associated with multidrug-resistant bacteria in secondary peritonitis: Impact on antibiotic therapy. Clin Microbiol Infect 2006;12:980-985.

18. Augustin P, Kermarrec N, Muller-Serieys C, et al. Risk factors for multidrug resistant bacteria and optimization of empirical antibiotic therapy in postoperative peritonitis. Crit Care 2010;14:R20.

19. Seguin P, Fédun Y, Laviolle B, et al. Risk factors for multidrug-resistant bacteria in patients with post-operative peritonitis requiring intensive care. J Antimicrob Chemother 2010;65:342-346.

20. Brook I. Bacterial studies of peritoneal cavity and postoperative surgical wound drainage following perforated appendix in children. Ann Surg 1980;192:208-212.

21. Brook I, Frazier EH. Aerobic and anaerobic microbiology in intra-abdominal infections associated with diverticulitis. J Med Microbiol 2000;49:827-830.

22. Dupont H, Carbon C, Carlet J. Monotherapy with a broadspectrum beta-lactam is as effective as its combination with an aminoglycoside in treatment of severe generalized peritonitis: A multicenter randomized controlled trial. Antimicrob Agents Chemother 2000;44:2028-2033.

23. Roehrborn A, Thomas L, Potreck O, et al. The microbiology of postoperative peritonitis. Clin Infect Dis 2001;33:1513-1519.

24. Montravers P, Lepape A, Dubreuil L, et al. Clinical and microbiological profiles of community-acquired and nosocomial intra-abdominal infections: Results of the French prospective, observational EBIIA study. J Antimicrob Chemother 2009;63:785-794.

25. Lee YL, Chen YS, Toh HS, et al. Antimicrobial susceptibility of pathogens isolated from patients with complicated intra-abdominal infections at five medical centers in Taiwan that continuously participated in the Study for Monitoring Antimicrobial Resistance Trends (SMART) from 2006 to 2010. Int J Antimicrob Agents 2012;40(Suppl):S29-S36.

26. Sartelli M, Catena F, Ansaloni, L, et al. Complicated intra-abdominal infections in Europe: A comprehensive review of the CIAO study. World J Emerg Surg 2012; 7:36.

27. van Ruler O, Kiewiet JJ, van Ketel RJ, et al. Initial microbial spectrum in severe secondary peritonitis and relevance for treatment. Eur J Clin Microbiol Infect Dis 2012;31:671-682.

28. Hawser S, Hoban DJ, Badal RE, et al. Epidemiology and antimicrobial susceptibility of Gram-negative aerobic 
bacteria causing intra-abdominal infections during 20102011. J Chemother 2015;27:67-73.

29. Hsueh PR. Study for monitoring antimicrobial resistance trends (SMART) in the Asia-Pacific region, 2002-2010. Int J Antimicrob Agents 2012;40(Suppl):S1-S3.

30. Lob SH, Badal RE, Bouchillon SK, et al. Epidemiology and susceptibility of Gram-negative appendicitis pathogens: SMART 2008-2010. Surg Infect 2013;14:203-208.

31. Yang Q, Zhang H, Wang Y, et al. A 10 year surveillance for antimicrobial susceptibility of Escherichia coli and Klebsiella pneumoniae in community- and hospitalassociated intra-abdominal infections in China. J Med Microbiol 2013;62:1343-1349.

32. Nathens AB, Rotstein OD, Marshall JC. Tertiary peritonitis: Clinical features of a complex nosocomial infection. World J Surg 1998;22:158-163.

33. Weiss G, Meyer F, Lippert H. Infectiological diagnostic problems in tertiary peritonitis. Langenbecks Arch Surg 2006;391:473-482.

34. Marshall JC. Intra-abdominal infections. Microbes Infect 2004;6:1015-1025.

35. Mazuski JE. Clinical challenges and unmet needs in the management of complicated intra-abdominal infections. Surg Infect (Larchmt) 2005;6(Suppl 2):S49-S69.

36. Dellinger EP, Wertz MJ, Meakins JL, et al. Surgical infection stratification system for intra-abdominal infection. Arch Surg 1985;120:21-29.

37. Billing A, Fröhlich D, Mialkowskyj O, et al. Treatment of peritonitis with staged lavage: Prognostic criteria and course of treatment. (Ger) Langenbecks Arch Chir 1992;377:305-313.

38. Christou NV, Barie PS, Dellinger EP, et al. Surgical Infection Society intra-abdominal infection study. Prospective evaluation of management techniques and outcome. Arch Surg 1993;128:193-198.

39. Hopkins JA, Lee JC, Wilson SE. Susceptibility of intraabdominal isolates at operation: A predictor of postoperative infection. Am Surg 1993;59:791-796.

40. Bohnen JM, Mustard RA, Schouten BD. Steroids, APACHE II score, and the outcome of abdominal infection. Arch Surg 1994:129;33-37.

41. Schoeffel U, Jacobs E, Ruf G, et al. Intraperitoneal microorganisms and the severity of peritonitis. Eur J Surg 1995;161:501-508.

42. Christou NV, Turgeon P, Wassef R, et al. Management of intra-abdominal infections. The case for intraoperative cultures and comprehensive broad-spectrum antibiotic coverage. Arch Surg 1996;131:1193-1201.

43. Montravers P, Gauzit R, Muller C, et al. Emergence of antibiotic resistant bacteria in cases of peritonitis after intraabdominal surgery affects the efficacy of empirical antimicrobial therapy. Clin Infect Dis 1996; 23:486-494.

44. Pacelli F, Doglietto GB, Alfieri S, et al. Prognosis in intraabdominal infections. Multivariate analysis on 604 patients. Arch Surg 1996;131:641-645.

45. Barie PS, Vogel SB, Dellinger EP, et al. A randomized, double-blind clinical trial comparing cefepime plus metronidazole with imipenem-cilastatin in the treatment of complicated intra-abdominal infections. Arch Surg 1997;132:1294-1302.

46. Bosscha K, Reijnders K, Hulstaert PF, et al. Prognostic scoring systems to predict outcome in peritonitis and intra-abdominal sepsis. Br J Surg 1997;84:1532-1534.
47. Ohmann C, Yang Q, Hau T, et al: Prognostic modelling in peritonitis. Eur J Surg 1997; 163:53-60.

48. Wacha H, Hau T, Dittmer R, Ohmann C. Risk factors associated with intraabdominal infections: A prospective multicenter study. Langenbecks Arch Surg 1999;384:24-32.

49. Sotto A, Lefrant JY, Fabbro-Peray P, et al. Evaluation of antimicrobial therapy management of 120 consecutive patients with secondary peritonitis. J Antimicrob Chemother 2002;50:569-576.

50. Holzheimer RG, Gathof B. Re-operation for complicated secondary peritonitis- how to identify patients at risk for persistent sepsis. Eur J Med Res 2003;8:125-134.

51. Mulier S, Penninckx F, Verwaest C, et al. Factors affecting mortality in generalized postoperative peritonitis: Multivariate analysis in 96 patients. World J Surg 2003;27:379-384.

52. Hutchins RR, Gunning MP, Lucas DL, et al. Relaparotomy for suspected intraperitoneal sepsis after abdominal surgery. World J Surg 2004;28:137-141.

53. Krobot K, Yin D, Zhang Q, et al. Effect of inappropriate initial empiric antibiotic therapy on outcome of patients with community-acquired intra-abdominal infections requiring surgery. Eur J Clin Microbiol Infect Dis 2004;23:682-687.

54. Sturkenboom MCJM, Goettsch WG, Picelli G, et al. Inappropriate initial treatment of secondary intra-abdominal infections leads to increased risk of clinical failure and costs. Br J Clin Pharmacol 2005;60:438-443.

55. Lamme B, Mahler CW, van Ruler O, et al. Clinical predictors of ongoing infection in secondary peritonitis: Systematic review. World J Surg 2006;30:2170-2181.

56. Montravers P, Dupont H, Gauzit R, et al. Candida as a risk factor for mortality in peritonitis. Crit Care Med 2006;34:646-652.

57. van Ruler O, Lamme B, Gouma DJ, et al. Variables associated with positive findings at relaparotomy in patients with secondary peritonitis. Crit Care Med 2007;35:468-476.

58. Gauzit R, Péan Y, Barth X, et al. Epidemiology, management, and prognosis of secondary non-postoperative peritonitis: A French prospective observational multicenter study. Surg Infect (Larchmt) 2009;10:119-127.

59. Inui T, Haridas M, Claridge JA, Malangoni MA. Mortality for intra-abdominal infection is associated with intrinsic risk factors rather than the source of infection. Surgery 2009;146:654-661.

60. Riché FC, Dray X, Laisné MJ, et al. Factors associated with septic shock and mortality in generalized peritonitis: Comparison between community-acquired and postoperative peritonitis. Crit Care 2009;13:R99.

61. Schneider CP, Seyboth C, Vilsmaier M, et al. Prognostic factors in critically ill patients suffering from secondary peritonitis: A retrospective, observational, survival time analysis. World J Surg 2009;33:34-43.

62. Swenson BR, Metzger R, Hedrick TL, et al. Choosing antibiotics for intra-abdominal infections: What do we mean by "high risk"? Surg Infect (Larchmt) 2009;10:29-39.

63. Kim JJ, Liang MK, Subramanian A, et al. Predictors of relaparotomy after nontrauma emergency general surgery with initial fascial closure. Am J Surg 2011;202:549-552.

64. Jean SS, Ko WC, Xie Y, et al. Clinical characteristics of patients with community-acquired complicated intraabdominal infections: A prospective, multicentre, observational study. Int J Antimicrob Agents 2014;44:222-228.

65. Pupelis G, Drozdova N, Mukans M, Malbrain ML. Serum procalcitonin is a sensitive marker for septic shock and 
mortality in secondary peritonitis. Anaesthesiol Intensive Ther 2014;46:262-273.

66. Bae JM. Clinical characteristics of ceftriaxone plus metronidazole in complicated intra-abdominal infection. Ann Surg Treat Res 2015;89:43-47.

67. Chong YP, Bae IG, Lee SR, et al. Clinical and economic consequences of failure of initial antibiotic therapy for patients with community-onset complicated intra-abdominal infections. PLoS One 2015;10:e119956.

68. Dupont H, Guilbart M, Ntouba A, et al. Can yeast isolation be predicted in complicated secondary non-postoperative intra-abdominal infections? Crit Care 2015;19:60.

69. Neri A, Marrelli D, Scheiterle M, et al. Re-evaluation of Mannheim prognostic index in perforative peritonitis: Prognostic role of advanced age. A prospective cohort study. Int J Surg 2015;13:54-59.

70. Suarez-de-la-Rica A, Maseda E, Anillo V, et al. Biomarkers (procalcitonin, $\mathrm{C}$ reactive protein, and lactate) as predictors of mortality in surgical patients with complicated intra-abdominal infection. Surg Infect (Larchmt) 2015;16:346-351.

71. Tellor B, Skrupky LP, Symons W, et al. Inadequate source control and inappropriate antibiotics are key determinants of mortality in patients with intra-abdominal sepsis and associated bacteremia. Surg Infect (Larchmt) 2015;16: 785-793.

72. Tridente A, Clarke GM, Walden A, et al. Association between trends in clinical variables and outcome in intensive care patients with faecal peritonitis: Analysis of the GenOSept cohort. Crit Care 2015;19:210.

73. Wu Q, Ren J, Wang G, et al. The incidence, clinical outcomes, and risk factors of thrombocytopenia in intraabdominal infection patients: A retrospective cohort study. PLoS One 2016;11:e0147482.

74. Vincent JL, Moreno R, Takala J, et al. Working group on sepsis-related problems of the European Society of Intensive Care Medicine. The SOFA (sepsis-related organ failure assessment) score to describe organ dysfunction/ failure. Intensive Care Med 1996;22:707-710.

75. Blomqvist PG, Andersson RE, Granath F, et al. Mortality after appendectomy in Sweden, 1987-1996. Ann Surg 2001;233:455-460.

76. Dumont R, Cinotti R, Lejus C, et al. The microbiology of community-acquired peritonitis in children. Pediatr Infect Dis J 2011;30:131-135.

77. Sawyer RG, Rosenlof LK, Adams RB, et al. Peritonitis into the 1990s: Changing pathogens and changing strategies in the critically ill. Am Surg 1992;58:82-87.

78. Koperna T, Schulz F. Prognosis and treatment of peritonitis. Do we need new scoring systems? Arch Surg 1996;131:180-186.

79. Panhofer P, Izay B, Riedl M, et al. Age, microbiology and prognostic scores help to differentiate between secondary and tertiary peritonitis. Langenbecks Arch Surg 2009; 394:265-271.

80. Singer M, Deutschman CS, Seymour CW, et al. The Third International Consensus Definitions for Sepsis and Septic Shock (Sepsis-3). JAMA 2016;315:801-810.

81. Koperna T, Schulz F. Relaparotomy in peritonitis: Prognosis and treatment of patients with persisting intraabdominal infection. World J Surg 2000;24:32-37.

82. Sawyer RG, Claridge JA, Nathens AB, et al. Trial of short-course antimicrobial therapy for intraabdominal infection. N Engl J Med 2015;372:1996-2005.
83. Billing A, Fröhlich D, Schildberg FW. Prediction of outcome using the Mannheim peritonitis index in 2003 patients. Peritonitis Study Group. Br J Surg 1994;81: 209-213.

84. Grunau G, Heemken R, Hau T. Predictors of outcome in patients with postoperative intra-abdominal infection. Eur J Surg 1996;162:619-625.

85. Rotstein OD, Pruett TL, Simmons RL. Microbiologic features and treatment of persistent peritonitis in patients in the intensive care unit. Can J Surg 1986;29:247-250.

86. Stiges-Serra A, López MJ, Girvent, M, et al. Postoperative enterococcal infection after treatment of complicated intra-abdominal sepsis. Br J Surg 2002;89:361-367.

87. Hasper D, Schefold JC, Baumgart DC. Management of severe abdominal infections. Recent Pat Antiinfect Drug Discov 2009;4:57-65.

88. Cercenado E, Torroba L, Cantón R, et al. Multicenter study evaluating the role of enterococci in secondary bacterial peritonitis. J Clin Microbiol 2010;48:456-459.

89. Dupont H, Friggeri A, Touzeau J, et al. Enterococci increase the morbidity and mortality associated with severe intra-abdominal infections in elderly patients hospitalized in the intensive care unit. J Antimicrob Chemother 2011; 66:2379-2385.

90. Friedrich AK, Cahan M. Intraabdominal infections in the intensive care unit. J Intensive Care Med 2014;29:247-254.

91. Peña C, Pujol M, Ardanuy C, et al. Epidemiology and successful control of a large outbreak due to Klebsiella pneumoniae producing extended-spectrum $\beta$-lactamases. Antimicrob Agents Chemother 1998;42:53-58.

92. Blot S, Depuydt P, Vogelaers D, et al. Colonization status and appropriate antibiotic therapy for nosocomial bacteremia caused by antibiotic-resistant gram-negative bacteria in an intensive care unit. Infect Control Hosp Epidemiol 2005;26:575-579.

93. Zirakzadeh A, Patel R. Vancomycin-resistant enterococci: Colonization, infection, detection, and treatment. Mayo Clin Proc 2006;81:529-536.

94. Klevens, RM, Morrison MA, Nadle J, et al. Invasive methicillin-resistant Staphylococcus aureus infections in the United States. JAMA 2007;298:1763-1771.

95. Reddy BS, Gatt M, Sowdi R, et al. Gastric colonization predisposes to septic morbidity in surgical patients: A prospective study. Nutrition 2008;24:632-637.

96. Tacconelli E, Cataldo MA. Vancomycin-resistant enterococci (VRE): Transmission and control. Int $\mathbf{J}$ Antimicrob Agents 2008;31:99-106.

97. Harinstein L, Schafer J, D'Amico F. Risk factors associated with the conversion of meticillin-resistant Staphylococcus aureus colonisation to healthcare-associated infection. J Hosp Infect 2011;79:194-197.

98. Schweickert B, Geffers C, Farragher T, et al. The MRSAimport in ICUs is an important predictor for the occurrence of nosocomial MRSA cases. Clin Microbiol Infect 2011;17:901-906.

99. Han JH, Nachamkin I, Zaoutis TE, et al. Risk factors for gastrointestinal tract colonization with extended-spectrum $\beta$-lactamase (ESBL)-producing Escherichia coli and Klebsiella species in hospitalized patients. Infect Cont Hosp Epidemiol 2012;33:1242-1245.

100. Tong SYC, Chen LF, Fowler VG Jr. Colonization, pathogenicity, host susceptibility, and therapeutics for Staphylococcus aureus: What is the clinical relevance? Semin Immunopathol 2012;34:185-200. 
101. Di Carlo P, Gulotta G, Casuccio A, et al. KPC - 3 Klebsiella pneumoniae ST258 clone infection in postoperative abdominal surgery patients in an intensive care setting: Analysis of a case series of 30 patients. BMC Anesthesiol 2013;13:13.

102. Orsi GB, Ciorba V. Vancomycin resistant enterococci healthcare associated infections. Ann Ig 2013;25:485-492.

103. Ziakas PD, Thapa R, Rice LB, Mylonakis E. Trends and significance of VRE colonization in the ICU: A metaanalysis of published studies. PLoS One 2013;8:e75658.

104. Giannella M, Trecarichi EM, De Rosa FG, et al. Risk factors for carbapenem-resistant Klebsiella pneumoniae bloodstream infection among rectal carriers: A prospective observational multicentre study. Clin Microbiol Infect 2014;20:1357-1562.

105. Lübbert C, Becker-Rux D, Rodloff AC, et al. Colonization of liver transplant recipients with KPC-producing Klebsiella pneumoniae is associated with high infection rates and excess mortality: A case-control analysis. Infection 2014;42:309-316.

106. O’Driscoll, Crank CW. Vancomycin-resistant enterococcal infections: Epidemiology, clinical manifestations, and optimal management. Infect Drug Resist 2015;8:217-230.

107. Bayer AS, Blumenkrantz MJ, Montgomerie JZ, et al. Candida peritonitis. Report of 22 cases and review of the English literature. Am J Med 1976;61:832-840.

108. Grewe M, Tsiotos GG, Luque de-Leon E, Sarr MG. Fungal infection in acute necrotizing pancreatitis. J Am Coll Surg 1999;188:408-414.

109. Isenmann R, Schwarz M, Rau B, et al. Characteristics of infection with Candida species in patients with necrotizing pancreatitis. World J Surg 2002;26:372-376.

110. Dupont H, Bourichon A, Paugam-Burtz C, et al. Can yeast isolation in peritoneal fluid be predicted in intensive care unit patients with peritonitis? Crit Care Med 2003;31: 752-757.

111. Harbarth S, Uckay I. Are there patients with peritonitis who require empiric therapy for enterococcus? Eur J Clin Microbiol Infect Dis 2004;23:73-77.

112. Cisneros JM, Rodríguez-Baño J, Fernández-Cuenca F, et al. Risk-factors for the acquisition of imipenemresistant Acinetobacter baumannii in Spain: A nationwide study. Clin Microbiol Infect 2005;11:874-879.

113. Georges B, Conil JM, Dubouix A, et al. Risk of emergence of Pseudomonas aeruginosa resistance to $\beta$-lactam antibiotics in intensive care units. Crit Care Med 2006; 34:1636-1641.

114. Peña C, Gudiol C, Tubau F, et al. Risk-factors for acquisition of extended-spectrum $\beta$-lactamase-producing Escherichia coli among hospitalised patients. Clin Microbiol Infect 2006;12:279-284.

115. Linden PK. Optimizing therapy for vancomycin-resistant enterococci (VRE). Semin Respir Crit Care Med 2007; 28:632-645.

116. Ben-Ami R, Rodríguez-Baño J, Arslan H, et al. A multinational survey of risk factors for infection with extendedspectrum $\beta$-lactamase-producing enterobacteriaceae in nonhospitalized patients. Clin Infect Dis 2009;49:682-690.

117. Fernández-Cuenca F, Gómez-Sánchez M, RodríguezBaño J, et al. Epidemiological and clinical features associated with colonisation/infection by Acinetobacter baumannii with phenotypic heterogeneous resistance to carbapenems. Int J Antimicrob Agents 2012;40:235-238.

118. Samuelsson A, Isaksson B, Chabok A, et al. Changes in the aerobic faecal flora of patients treated with antibiotics for acute intra-abdominal infection. Scand J Infect Dis 2012;44:820-827.

119. Seguin P, Brianchon C, Launey Y, et al. Are enterococci playing a role in postoperative peritonitis in critically ill patients? Eur J Clin Microbiol Infect Dis 2012;31: 1479-1485.

120. Siedelman L, Kline S, Duval S. Risk factors for community- and health facility-acquired extended-spectrum $\beta$ lactamase-producing bacterial infections in patients at the University of Minnesota Medical Center, Fairview. Am J Infect Cont 2012;40:849-853.

121. Liew YX, Tan TT, Lee W, et al. Risk factors for extremedrug resistant Pseudomonas aeruginosa infections in patients with hematologic malignancies. Am J Infect Cont 2013;41:140-144.

122. Cohen MJ, Block CS, Moses AE, Nir-Paz R. Exposure to ertapenem is possibly associated with Pseudomonas aeruginosa antibiotic resistance. Clin Microbiol Infect 2014;20:O188-O196.

123. Tumbarello T, Trecarichi EM, Tumietto F, et al. Predictive models for identification of hospitalized patients harboring KPC-producing Klebsiella pneumoniae. Antimicrob Agents Chemother 2014;58:3514-3520.

124. Chalmers JD, Rother C, Salih W, Ewig S. Healthcareassociated pneumonia does not accurately identify potentially resistant pathogens: A systematic review and meta-analysis. Clin Infect Dis 2014;58:330-339.

125. American Thoracic Society, Infectious Diseases Society of America. Guidelines for the management of adults with hospital-acquired, ventilator-associated, and healthcareassociated pneumonia. Am J Respir Crit Care Med 2005; 171:388-416.

126. Bohnen JM, Marshall JC, Fry DE, et al. Clinical and scientific importance of source control in abdominal infections: Summary of a symposium. Can J Surg 1999;42:122-126.

127. Marshall JC, Innes M. Intensive care unit management of intra-abdominal infection. Crit Care Med 2003;31:22282237.

128. Marshall JC, Maier RV, Jimenez M, Dellinger EP. Source control in the management of severe sepsis and septic shock: An evidence-based review. Crit Care Med 2004; 32[Suppl]:S513-S526.

129. Pieracci FM, Barie PS. Intra-abdominal infections. Curr Opin Crit Care 2007;13:440-449.

130. Weigelt JA. Empiric treatment options in the management of complicated intra-abdominal infections. Cleve Clin J Med 2007;74(Suppl 4);S29-S37.

131. Andeweg CS, Mulder IM, Felt-Bersma RJ, et al. Guidelines of diagnostics and treatment of acute left-sided colonic diverticulitis. Dig Surg 2013;30:278-292.

132. Feingold D, Steele SR, Lee S, et al. Practice parameters for the treatment of sigmoid diverticulitis. Dis Colon Rectum 2014;57:284-294.

133. Nadler EP, Reblock KK, Vaughan KG, et al. Predictors of outcome for children with perforated appendicitis initially treated with non-operative management. Surg Infect (Larchmt) 2004;5:349-356.

134. Andersson RE, Petzold MG. Nonsurgical treatment of appendiceal abscess or phlegmon: A systematic review and meta-analysis. Ann Surg 2007;246:741-748.

135. Simillis C, Symeonides P, Shorthouse AJ, Tekkis PP. A meta-analysis comparing conservative treatment versus acute appendectomy for complicated appendicitis (abscess or phlegmon). Surgery 2010;147:818-829. 
136. Crofts, TJ, Park KG, Steele RJ, et al. A randomized trial of nonoperative treatment for perforated peptic ulcer. N Engl J Med 1989;320:970-973.

137. Marshall C, Ramaswamy P, Bergin FG, et al. Evaluation of a protocol for the non-operative management of perforated peptic ulcer. Br J Surg 1999;86:131-134.

138. Cao F, Li J, Li A, et al. Nonoperative management for perforated peptic ulcer: Who can benefit? Asian J Surg 2014;37:148-153.

139. Jeffrey RB, Federle MP, Tolentino CS. Periappendiceal inflammatory masses: CT-directed management and clinical outcome in 70 patients. Radiology 1988;167:13-16.

140. Brandt D, Gervaz P, Durmishi Y, et al. Percutaneous CT scan-guided drainage vs. antibiotherapy alone for Hinchey II diverticulitis: A case-control study. Dis Colon Rectum 2006;49:1533-1538.

141. Siewert B, Tye G, Kruskal J, et al. Impact of CT-guided drainage in the treatment of diverticular abscesses: Size matters. AJR Am J Roentgenol 2006;186:680-686.

142. Dharmarajan S, Hunt SR, Birnbaum EH, et al. The efficacy of nonoperative management of acute complicated diverticulitis. Dis Colon Rectum 2011;54:663-671.

143. Bakker OJ, van Santvoort HC, Besselink MG, et al. Prevention, detection, and management of infected necrosis in severe acute pancreatitis. Curr Gastroenterol Rep 2009; 11:104-110.

144. Mouli VP, Sreenivas V, Garg PK. Efficacy of conservative treatment, without necrosectomy, for infected pancreatic necrosis: A systematic review and meta-analysis. Gastroenterology 2013;144:333-340.

145. Mentula P, Leppäniemi A. Position paper: Timely interventions in severe acute pancreatitis are crucial for survival. World J Emerg Surg 2014;9:15.

146. De Waele JJ. Early source control in sepsis. Langenbecks Arch Surg 2010;395:489-494.

147. Bloos F, Thomas-Rüddel D, Rüddel H, et al. Impact of compliance with infection management guidelines on outcome in patients with severe sepsis: A prospective observational multi-center study. Crit Care 2014;18:R42.

148. Hecker A, Schneck E, Röhrig R, et al. The impact of early surgical intervention in free intestinal perforation: A time-tointervention pilot study. World J Emerg Surg 2015;10:54.

149. van Goor H. Interventional management of abdominal sepsis: When and how. Langenbecks Arch Surg 2002; 387:191-200.

150. Betsch A, Wiskirchen J, Trübenbach J, et al. CT-guided percutaneous drainage of intra-abdominal abscesses: APACHE III score stratification of 1-year results. Eur Radiol 2002;12:2883-2889.

151. Cinat ME, Wilson SE, Din AM. Determinants for successful percutaneous image-guided drainage of intraabdominal abscess. Arch Surg 2002;137:845-849.

152. Akinci D, Akhan O, Ozmen $\mathrm{MN}$, et al. Percutaneous drainage of 300 intraperitoneal abscesses with long-term follow-up. Cardiovasc Intervent Radiol 2005;28:744-750.

153. Theisen J, Bartels H, Weiss W, et al. Current concepts of percutaneous abscess drainage in postoperative retention. J Gastrointest Surg 2005;9:280-283.

154. Alamili M, Gögenur, I, Rosenberg J. Acute complicated diverticulitis managed by laparoscopic lavage. Dis Colon Rectum 2009;52:1345-1349.

155. Toorenvliet BR, Swank H, Schoones JW, et al. Laparoscopic peritoneal lavage for perforated colonic diverticulitis: A systematic review. Colorectal Dis 2010;12:862-867.
156. Rossi GL, Mentz R, Bertone S, et al. Laparoscopic peritoneal lavage for Hinchey III diverticulitis: Is it as effective as it is applicable? Dis Colon Rectum 2014;57:1384-1390.

157. Cirocchi R, Trastulli S, Vettoretto N, et al. Laparoscopic peritoneal lavage: A definitive treatment for diverticular peritonitis or a "bridge" to elective laparoscopic sigmoidectomy? A systematic review. Medicine (Baltimore) 2015;94:e334.

158. Schultz JK, Yaqub S, Wallon C, et al. Laparoscopic lavage vs primary resection for acute perforated diverticulitis. The SCANDIV randomized clinical trial. JAMA 2015;314:1364-1375.

159. Vennix S, Musters GD, Mulder IM, et al. Laparoscopic peritoneal lavage or sigmoidectomy for perforated diverticulitis with purulent peritonitis: A multicentre, parallelgroup, randomised, open-label trial. Lancet 2015:386: 1269-1277.

160. Kiewiet JJ, van Ruler O, Boermeester MA, Reitsma JB. A decision rule to aid selection of patients with abdominal sepsis requiring a relaparotomy. BMC Surg 2013;13:28.

161. Ordoñez CA, Puyana JC. Management of peritonitis in the critically ill patient. Surg Clin North Am 2006;86:13231349.

162. Weber DG, Bendinelli C, Balogh ZJ. Damage control surgery for abdominal emergencies. Br J Surg 2014;101: e109-e118.

163. Diaz JJ Jr, Cullinane DC, Dutton WD, et al. The management of the open abdomen in trauma and emergency general surgery: Part 1-Damage control. J Trauma 2010;68:1425-1438.

164. Waibel BH, Rotondo MF. Damage control for intraabdominal sepsis. Surg Clin North Am 2012;92:243-257.

165. Balogh ZJ, Leppäniemi A. Patient populations at risk for intra-abdominal hypertension and abdominal compartment syndrome. Am Surg 2011;77(Suppl 1):S12-S16.

166. Schein M. Surgical management of intra-abdominal infection: Is there any evidence? Langenbecks Arch Surg 2002;387:1-7.

167. Lee JC, Peitzman AB. Damage-control laparotomy. Curr Opin Crit Care 2006;12:346-350.

168. Stawicki SP, Brooks A, Bilski T, et al. The concept of damage control: Extending the paradigm to emergency general surgery. Injury 2008;39:93-101.

169. Khan A, Hsee L, Mathur S, Civil I. Damage-control laparotomy in nontrauma patients: Review of indications and outcomes. J Trauma Acute Care Surg 2013;75:365-368.

170. van Ruler O, Mahler CW, Boer KR, et al. Comparison of on-demand vs planned relaparotomy strategy in patients with severe peritonitis: A randomized trial. JAMA 2007;298:865-872.

171. Opmeer BC, Boer KR, van Ruler O, et al. Costs of relaparotomy on-demand versus planned relaparotomy in patients with severe peritonitis: An economic evaluation within a randomized controlled trial. Crit Care 2010; 14:R97.

172. Seiler CA, Brügger L, Forssmann U, et al. Conservative surgical treatment of diffuse peritonitis. Surgery 2000;127:178-184.

173. Lamme B, Boermeester MA, Belt EJ, et al. Mortality and morbidity of planned relaparotomy versus relaparotomy on demand for secondary peritonitis. $\mathrm{Br} \mathrm{J}$ Surg 2004;91:1046-1054.

174. Robledo FA, Luque-de-León E, Suárez R, et al. Open versus closed management of the abdomen in the surgical 
treatment of severe secondary peritonitis: A randomized clinical trial. Surg Infect 2007;8:63-72.

175. Zhang WB, Li N, Wang PF, et al. Infections following damage control laparotomy with abdominal packing. Scand J Infect Dis 2008;40:867-876.

176. Moussavian MR, Richter S, Kollmar O, et al. Staged lavage versus single high-volume lavage in the treatment of feculent/purulent peritonitis: A matched pair analysis. Langenbecks Arch Surg 2009;394:215-220.

177. Mandell K, Arbabi S. Re-laparotomy for severe intraabdominal infections. Surg Infect (Larchmt) 2010;11: 307-310.

178. Platell C, Papadimitriou JM, Hall JC. The influence of lavage on peritonitis. J Am Coll Surg 2000;191:672-680.

179. Moore CB, Smith RS, Herbertson R, Toevs C. Does use of intraoperative irrigation with open or laparoscopic appendectomy reduce post-operative intra-abdominal abscess? Am Surg 2011;77:78-80.

180. Akkoyun I, Tuna AT. Advantages of abandoning abdominal cavity irrigation and drainage in operations performed on children with perforated appendicitis. J Pediatr Surg 2012;47:1886-1890.

181. St. Peter SD, Adibe OO, Iqbal CW, et al. Irrigation versus suction alone during laparoscopic appendectomy for perforated appendicitis: A prospective randomized trial. Ann Surg 2012;256:581-585.

182. Hartwich JE, Carter RF, Wolfe L, et al. The effects of irrigation on outcomes in cases of perforated appendicitis in children. J Surg Res 2013;180:222-225.

183. Leiboff AR, Soroff HS. The treatment of generalized peritonitis by closed postoperative peritoneal lavage. A critical review of the literature. Arch Surg 1987;122: 1005-1010.

184. Suding PN, Orrico RP, Johnson SB, Wilson SE. Concordance of interrater assessments of surgical methods to achieve source control of intra-abdominal infections. Am J Surg 2008;196:70-73.

185. Solomkin JS, Ristagno RL, Das AF, et al. Source control review in clinical trials of anti-infective agents in complicated intra-abdominal infections. Clin Infect Dis 2013;56:1765-1773.

186. Davies HO, Alkhamesi NA, Dawson PM. Peritoneal fluid culture in appendicitis: Review in changing times. Int $\mathbf{J}$ Surg 2010;8:426-429.

187. Mosdell DM, Morris DM, Voltura A, et al. Antibiotic treatment for surgical peritonitis. Ann Surg 1991;214:543-549.

188. Kokoska ER, Silen ML, Tracy TF Jr, et al. The impact of intraoperative culture on treatment and outcome in children with perforated appendicitis. J Pediatr Surg 1999;34:749-753.

189. Çelik A, Ergün O, Ozcan C, et al. Is it justified to obtain routine peritoneal cultures during appendectomy in children? Pediatr Surg Int 2003;19:632-634.

190. Gladman MA, Knowles CH, Gladman LJ, Payne JG. Intra-operative culture in appendicitis: Traditional practice challenged. Ann R Coll Surg Engl 2004;86:196-201.

191. Khan MN, Vidya R, Lee RE. Are routine peritoneal fluid cultures during appendicectomy justified? Ir J Med Sci 2007;176:37-40.

192. Edelsberg J, Berger A, Schell S, et al. Economic consequences of failure of initial antibiotic therapy in hospitalized adults with complicated intra-abdominal infections. Surg Infect (Larchmt) 2008;9:335-347.

193. Kollef MH, Sherman, G, Ward S, Fraser VJ. Inadequate antimicrobial treatment of infections: A risk factor for hospital mortality among critically ill patients. Chest 1999;115:462-474.

194. Garnacho-Montero J, Ortiz-Leyba C, Herrera-Melero, I, et al. Mortality and morbidity attributable to inadequate empirical antimicrobial therapy in patients admitted to the ICU with sepsis: A matched cohort study. J Antimicrob Chemother 2008;61:436-441.

195. Paul M, Shani V, Muchtar E, et al. Systematic review and meta-analysis of the efficacy of appropriate empiric antibiotic therapy for sepsis. Antimicrob Agents Chemother 2010;54:4851-4863.

196. Dellit TH, Owens RC, McGowan JE Jr., et al. Infectious Diseases Society of America and the Society for Healthcare Epidemiology of America guidelines for developing an institutional program to enhance antimicrobial stewardship. Clin Infect Dis 2007;44:159-177.

197. Cotta MO, Roberts JA, Tabah A, et al. Antimicrobial stewardship of $\beta$-lactams in intensive care units. Expert Rev Anti Infect Ther 2014;12:581-595.

198. Garnacho-Montero J, Gutiérrez-Pizarraya A, EscorescaOrtega A, et al. De-escalation of empirical therapy is associated with lower mortality in patients with severe sepsis and septic shock. Intensive Care Med 2014;40:32-40.

199. Davey PG, Marwick C. Appropriate vs. inappropriate antimicrobial therapy. Clin Microbiol Infect 2008;14(Suppl 3): $15-21$.

200. MacGowan AP; BSAC Working Parties on Resistance Surveillance. Clinical implications of antimicrobial resistance for therapy. J Antimicrob Chemother 2008;62(Suppl 2):ii105-ii114.

201. Heseltine PN, Yellin AE, Appleman MD, et al. Perforated and gangrenous appendicitis: An analysis of antibiotic failures. J Infect Dis 1983;148:322-329.

202. Baré M, Castells X, Garcia A, et al. Importance of appropriateness of empiric antibiotic therapy on clinical outcomes in intra-abdominal infections. Int $\mathrm{J}$ Technol Assess Health Care 2006;22:242-248.

203. Chandra A, Dhar P, Dharap S, et al. Cefoperazonesulbactam for treatment of intra-abdominal infections: Results from a randomized, parallel group study in India. Surg Infect (Larchmt) 2008;9:367-376.

204. St. Peter SD, Tsao K, Spilde TL, et al. Single daily dosing ceftriaxone and metronidazole vs standard triple antibiotic regimen for perforated appendicitis in children: A prospective randomized trial. J Pediatr Surg 2008;43:981-985.

205. Dalgic N, Karadag CA, Bayraktar B, et al. Ertapenem versus standard triple antibiotic therapy for the treatment of perforated appendicitis in pediatric patients: A prospective randomized trial. Eur J Pediatr Surg 2014; 24:410-418.

206. Pérez V, Saénz D, Madriz J, et al. A double-blind study of the efficacy and safety of multiple daily doses of amikacin versus one daily dose for children with perforated appendicitis in Costa Rica. Int J Infect Dis 2011;15:e569-e575.

207. Bailey JA, Virgo KS, DiPiro JT, et al. Aminoglycosides for intra-abdominal infection: Equal to the challenge? Surg Infect (Larchmt) 2002;3:315-335.

208. Falagas ME, Matthaiou DK, Karveli EA, Peppas G. Metaanalysis: Randomized controlled trials of clindamycin/ aminoglycoside vs. $\beta$-lactam monotherapy for the treatment of intra-abdominal infections. Aliment Pharmacol Ther 2007;25:537-556.

209. Jauregui LE, Appelbaum PC, Fabian TC, et al. A randomized clinical study of cefoperazone and sulbactam 
versus gentamicin and clindamycin in the treatment of intra-abdominal infections. $\mathrm{J}$ Antimicrob Chemother 1990;25:423-433.

210. Kooi GH, Pit S. Ceftazidime/metronidazole versus netilmicin/metronidazole in the treatment of perforated appendicitis in children. Clin Ther 1990;12:54-60.

211. Solomkin JS, Dellinger EP, Christou NV, Busuttil RW. Results of a multicenter trial comparing imipenem/cilastatin to tobramycin/clindamycin for intra-abdominal infections. Ann Surg 1990;212:581-591.

212. Luke M, Iversen J, Søndergaard J, et al. Ceftriaxone/ metronidazole is more effective than ampicillin/netilmicin/metronidazole in the treatment of bacterial peritonitis. Eur J Surg 1991;157:397-401.

213. Yellin AE, Heseltine PN, Berne TV, et al. The role of Pseudomonas species in patients treated with ampicillin and sulbactam for gangrenous and perforated appendicitis. Surg Gynecol Obstet 1985;161:303-307.

214. Catena F, Vallicelli C, Ansaloni L, et al. T.E.A. study: Threeday ertapenem versus three-day ampicillin-sulbactam. BMC Gastroenterol 2013;13:76.

215. Chen CW, Ming CC, Ma CJ, et al. Prospective, randomized, study of ampicillin-sulbactam versus moxifloxacin monotherapy for the treatment of community-acquired complicated intra-abdominal infections. Surg Infect (Larchmt) 2013;14:389-396.

216. Betrosian AP, Douzinas EE. Ampicillin-sulbactam: An update on the use of parenteral and oral forms in bacterial infections. Expert Opin Drug Metab Toxicol 2009;5: 1099-1112.

217. Yoshioka K, Youngs DJ, Keighley MR. A randomized prospective controlled study of ciprofloxacin with metronidazole versus amoxicillin/clavulanic acid with metronidazole in the treatment of intra-abdominal infection. Infection 1991;19:25-29.

218. Rahnama'1 MS, Wagenvoort JH, van der Linden CJ. Amoxicillin/clavulanate (Augmentin) resistant Escherichia coli in bacterial peritonitis after abdominal surgery-clinical outcome in ICU patients. Neth J Med 2009;67:173-176.

219. Kacmaz B, Sultan N. In vitro susceptibilities of Escherichia coli and Klebsiella spp. to ampicillin-sulbactam and amoxicillin-clavulanic acid. Jpn J Infect Dis 2007;60:227229.

220. Yellin AE, Johnson J, Higareda I, et al. Ertapenem or ticarcillin/clavulanate for the treatment of intra-abdominal infections or acute pelvic infections in pediatric patients. Am J Surg 2007;194:367-374.

221. Jones RN, Stilwell MG, Rhomberg PR, Sader HS. Antipseudomonal activity of piperacillin/tazobactam: More than a decade of experience from the SENTRY Antimicrobial Surveillance Program (1997-2007). Diag Microbiol Infect Dis 2009;65:331-334.

222. Higgins PG, Wisplinghoff $H$, Stefanik D, Seifert $H$. In vitro activities of the $\beta$-lactamase inhibitors clavulanic acid, sulbactam, and tazobactam alone or in combination with $\beta$-lactams against epidemiologically characterized multidrug-resistant Acinetobacter baumannii strains. Antimicrob Agents Chemother 2004;48:15861592.

223. Payne DJ, Cramp R, Winstanley DJ, Knowles DJ. Comparative activities of clavulanic acid, sulbactam, and tazobactam against clinically important $\beta$-lactamases. Antimicrob Agents Chemother 1994;38:767-772.
224. American Society of Health-System Pharmacists. Ticarcillin clavulanate. 2015; www.ashp.org/menu/DrugShortages/ DrugsNoLongerAvailable/Bulletin.aspx $? \mathrm{id}=802$. (Last accessed October 2, 2015).

225. Maltezou HC, Nikolaidis P, Lebesii E, et al. Piperacillin/ tazobactam versus cefotaxime plus metronidazole for treatment of children with intra-abdominal infections requiring surgery. Eur J Clin Microbiol Infect Dis 2001; 20:643-646.

226. Solomkin JS, Yellin AE, Rotstein OD, et al. Ertapenem versus piperacillin/tazobactam in the treatment of complicated intraabdominal infections: Results of a doubleblind, randomized comparative phase III trial. Ann Surg 2003;237:235-245.

227. Erasmo AA, Crisostomo AC, Yan LN, et al. Randomized comparison of piperacillin/tazobactam versus imipenem/ cilastatin in the treatment of patients with intra-abdominal infection. Asian J Surg 2004;27:227-235.

228. Dela Pena AS, Asperger W, Köckerling F, et al. Efficacy and safety of ertapenem versus piperacillin-tazobactam for the treatment of intra-abdominal infections requiring surgical intervention. J Gastroint Surg 2006;10:567-574.

229. Malangoni, MA, Song J, Herrington J, et al. Randomized controlled trial of moxifloxacin compared with piperacillin-tazobactam and amoxicillin-clavulanate for the treatment of complicated intra-abdominal infections. Ann Surg 2006;244:204-211.

230. Namias N, Solomkin JS, Jensen EH, et al. Randomized, multicenter, double-blind study of efficacy, safety, and tolerability of intravenous ertapenem versus piperacillin/ tazobactam in treatment of complicated intra-abdominal infections in hospitalized adults. Surg Infect (Larchmt) 2007;8:15-28.

231. Itani KM, Wilson SE, Awad SS, et al. Ertapenem versus cefotetan prophylaxis in elective colorectal surgery. $\mathrm{N}$ Engl J Med 2006;355:2640-2651.

232. Deierhoi RJ, Dawes LG, Vick C, et al. Choice of intravenous antibiotic prophylaxis for colorectal surgery does matter. J Am Coll Surg 2013;217:763-769.

233. Hendren S, Fritze D, Banerjee M, et al. Antibiotic choice is independently associated with risk of surgical site infection after colectomy. A population-based cohort study. Ann Surg 2013;257:469-475.

234. Babinchak T, Badal R, Hoban D, et al. Trends in susceptibility of selected gram-negative bacilli isolated from intra-abdominal infections in North America: SMART 2005-2010. Diagn Microbiol Infect Dis 2013;76:379-381.

235. Villegas MV, Blanco MG, Sifuentes-Osornio J, Rossi F. Increasing prevalence of extended-spectrum-betalactamase among Gram-negative bacilli in Latin America -2008 update from the Study for Monitoring Antimicrobial Resistance Trends (SMART). Braz J Infect Dis 2011;15:34-39.

236. Boyanova L, Kolarov R, Mitov I. Recent evolution of antibiotic resistance in the anaerobes as compared to previous decades. Anaerobe 2015; 31:4-10.

237. Claros M, Citron DM, Goldstein EJ, et al. Differences in distribution and antimicrobial susceptibility of anaerobes isolated from complicated intra-abdominal infections versus diabetic foot infections. Diag Microbiol Infect Dis 2013;76:546-548.

238. Bratzler DW, Dellinger EP, Olsen KM, et al. Clinical practice guidelines for antimicrobial prophylaxis in surgery. Surg Infect (Larchmt) 2013;14:73-156. 
239. Falagas ME, Karageorgopoulos DE. Extended-spectrum $\beta$-lactamase-producing organisms. J Hosp Infect 2009;73: 345-354.

240. el-Sefi TA, el-Awady HM, Shehata MI, al-Hindi MA. Systemic plus local metronidazole and cephazolin in complicated appendicitis: A prospective controlled trial. J R Coll Surg Edinb 1989;34:13-16.

241. Chen CY, Chen YC, Pu HN, et al. Bacteriology of acute appendicitis and its implication for the use of prophylactic antibiotics. Surg Infect (Larchmt) 2012;13:383-390.

242. Schito GC, Naber KG, Botto H, et al. The ARESC study: An international survey on the antimicrobial resistance of pathogens involved in uncomplicated urinary tract infections. Int J Antimicrob Agents 2009;34:407-413.

243. Huizinga WK, Warren BL, Baker LW, et al. Antibiotic monotherapy with meropenem in the surgical management of intra-abdominal infections. J Antimicrob Chemother 1995;36(Suppl A):179-189.

244. Kempf P, Bauernfeind A, Müller A, Blum J. Meropenem monotherapy versus cefotaxime plus metronidazole combination treatment for serious intra-abdominal infections. Infection 1996;24:473-479.

245. Hoogkamp-Korstanje JA. Ciprofloxacin vs. cefotaxime regimens for the treatment of intra-abdominal infections. Infection 1995;23:278-282.

246. Yellin AE, Hassett JM, Fernandez A, et al. Ertapenem monotherapy versus combination therapy with ceftriaxone plus metronidazole for treatment of complicated intraabdominal infections in adults. Int $\mathbf{J}$ Antimicrob Agents 2002;20:165-173.

247. Starakis I, Karravias D, Asimakopoulos C, et al. Results of a prospective, randomized, double blind comparison of the efficacy and the safety of sequential ciprofloxacin (intravenous/oral) + metronidazole (intravenous/oral) with ceftriaxone (intravenous) +/metronidazole (intravenous/ oral) for the treatment of intra-abdominal infections. Int $\mathrm{J}$ Antimicrobial Agents 2003;21:49-57.

248. Navarro NS Jr, Campos MI, Alvarado R, et al. Ertapenem versus ceftriaxone and metronidazole as treatment for complicated intra-abdominal infections. Int J Surg 2005;3:25-34.

249. Wacha H, Warren B, Bassaris H, et al. Comparison of sequential intravenous/oral ciprofloxacin plus metronidazole with intravenous ceftriaxone plus metronidazole for treatment of complicated intra-abdominal infections. Surg Infect (Larchmt) 2006; 7:341-354.

250. Solomkin J, Zhao YP, Ma EL, et al. Moxifloxacin is noninferior to combination therapy with ceftriaxone plus metronidazole in patients with community-origin complicated intra-abdominal infections. Int $\mathrm{J}$ Antimicrobial Agents 2009;34:439-445.

251. Weiss G, Reimnitz P, Hampel B, et al. Moxifloxacin for the treatment of patients with complicated intraabdominal infections (the AIDA Study). J Chemother 2009;21:170-180.

252. Towfigh S, Pasternak J, Poirier, A. A multicentre, openlabel, randomized comparative study of tigecycline versus ceftriaxone sodium plus metronidazole for the treatment of hospitalized subjects with complicated intra-abdominal infections. Clin Microbiol Infect 2010;16:1274-1281.

253. Qvist N, Warren B, Leister-Tebbe H, et al. Efficacy of tigecycline versus ceftriaxone plus metronidazole for the treatment of complicated intra-abdominal infections: Results from a randomized, controlled trial. Surg Infect (Larchmt) 2012;13:102-109.
254. Badaró R, Molinar F, Seas C, et al. A multicenter comparative study of cefepime versus broad-spectrum antibacterial therapy in moderate and severe bacterial infections. Braz J Infect Dis 2002;6:206-218.

255. Garbino J, Villiger P, Caviezel A, et al. A randomized prospective study of cefepime plus metronidazole with imipenem-cilastatin in the treatment of intra-abdominal infections. Infection 2007;35:161-166.

256. Jacoby GA. AmpC $\beta$-lactamases. Clin Microbiol Rev 2009; 22:161-182.

257. Harris PN, Tambyah PA, Paterson DL. $\beta$-Lactam and $\beta$ lactamase inhibitor combinations in the treatment of extendedspectrum $\beta$-lactamase producing Enterobacteriaceae: Time for a reappraisal in the era of few antibiotic options? Lancet Infect Dis 2015;15:475-485.

258. Tamma PD, Girdwood SCT, Gopaul R, et al. The use of cefepime for treating AmpC $\beta$-lactamase-producing Enterobacteriaceae. Clin Infect Dis 2013;57:781-788.

259. Blanchette LM, Kuti JL, Nicolau DP, Nailor MD. Clinical comparison of ertapenem and cefepime for treatment of infections caused by AmpC beta-lactamase-producing Enterobacteriaceae. Scand J Infect Dis 2014;46:803-808.

260. Ishii Y, Ueda C, Kouyama Y, et al. Evaluation of antimicrobial susceptibility for $\beta$-lactams against clinical isolates from 51 medical centers in Japan (2008). Diag Microbiol Infect Dis 2011;69:443-448.

261. Jones RN, Guzman-Blanco M, Gales AC, et al. Susceptibility rates in Latin American nations: Report from a regional resistance surveillance program (2011). Braz J Infect Dis 2013;17:672-681.

262. Jones RN, Castanheira M, Hu B, et al. Update of contemporary antimicrobial resistance rates across China: Reference testing results for 12 medical centers (2011). Diagn Microbiol Infect Dis 2013;77:258-266.

263. Lucasti C, Hershberger E, Miller M, et al. Multicenter, double-blind, randomized, phase II trial to assess the safety and efficacy of ceftolozane-tazobactam plus metronidazole compared with meropenem in adult patients with complicated intra-abdominal infections. Antimicrob Agents Chemother 2014;58:5350-5357.

264. Solomkin J, Hershberger E, Miller B, et al. Ceftolozane/ tazobactam plus metronidazole for complicated intraabdominal infections in an era of multidrug resistance: Results from a randomized, double-blind, phase 3 trial (ASPECT-cIAI). Clin Infect Dis 2015;60:1462-1471.

265. Maseda E, Aguilar L, Gimenez MJ, Gilsanz F. Ceftolozane/tazobactam (CXA 201) for the treatment of intraabdominal infections. Exp Review Anti Infect Ther 2014;12:1311-1324.

266. Zhanel GG, Chung P, Adam H, et al. Ceftolozane/tazobactam: A novel cephalosporin/ $\beta$ - lactamase inhibitor combination with activity against multidrug-resistant gram-negative bacilli. Drugs 2014;74:31-51.

267. Cho JC, Fiorenza MA, Estrada SJ. Ceftolozane/tazobactam: A novel cephalosporin/ $\beta$-lactamase inhibitor combination. Pharmacotherapy 2015;35:701-715.

268. Liscio JL, Mahoney MV, Hirsch EB. Ceftolozane/tazobactam and ceftazidime/avibactam: Two novel $\beta$-lactam/ $\beta$-lactamase inhibitor combination agents for the treatment of resistant Gram-negative bacterial infections. Int $\mathbf{J}$ Antimicrob Agents 2015;46:266-271.

269. Walkty A, Karlowsky JA, Adam H, et al. In vitro activity of ceftolozane-tazobactam against Pseudomonas aeruginosa isolates obtained from patients in Canadian hospitals 
in the CANWARD study, 2007 to 2012. Antimicrob Agents Chemother 2013;57:5707-5709.

270. Farrell DJ, Flamm RK, Sader HS, Jones RN. Antimicrobial activity of ceftolozane-tazobactam tested against Enterobacteriaceae and Pseudomonas aeruginosa with various resistance patterns isolated in U.S. hospitals (2011-2012). Antimicrob Agents Chemother 2013;57:6305-6310.

271. Farrell DJ, Sader HS, Flamm RK, Jones RN. Ceftolozane/ tazobactam activity tested against Gram-negative bacterial isolates from hospitalised patients with pneumonia in US and European medical centres (2012). Int J Antimicrob Agents 2014;43:533-539.

272. Sader HS, Farrell DJ, Castanheira M, et al. Antimicrobial activity of ceftolozane/tazobactam tested against Pseudomonas aeruginosa and Enterobacteriaceae with various resistance patterns isolated in European hospitals (201112). J Antimicrob Chemother 2014;69:2713-2722.

273. Sader HS, Farrell DJ, Flamm RK, Jones RN. Ceftolozane/ tazobactam activity tested against aerobic Gram-negative organisms isolated from intra-abdominal and urinary tract infections in European and United States hospitals (2012). J Infect 2014;69:266-277.

274. Lucasti C, Popescu I, Ramesh MK, et al. Comparative study of the efficacy and safety of ceftazidime/avibactam plus metronidazole versus meropenem in the treatment of complicated intra-abdominal infections in hospitalized adults: Results of a randomized, double-blind, Phase II trial. J Antimicrob Chemother 2013;68:1183-1192.

275. Mazuski JE, Gasink LB, Armstrong J, et al. Efficacy and safety of ceftazidime-avibactam plus metronidazole versus meropenem in the treatment of complicated intraabdominal infection: Results from a randomized, controlled, double-blind, phase 3 program. Clin Infect Dis 2016;62:1380-1389.

276. Zhanel GG, Lawson CD, Adam H, et al. Ceftazidimeavibactam: A novel cephalosporin/ $\beta$-lactamase inhibitor combination. Drugs 2013;73:159-177.

277. Lagacé-Wiens P, Walkty A, Karlowsky JA. Ceftazidimeavibactam: An evidence-based review of its pharmacology and potential use in the treatment of Gram-negative bacterial infections. Core Evid 2014;9:13-25.

278. Sader HS, Castanheira M, Flamm RK, et al. Ceftazidime/ avibactam tested against Gram-negative bacteria from intensive care unit (ICU) and non-ICU patients, including those with ventilator-associated pneumonia. Int $\mathrm{J}$ Antimicrob Agents 2015;46:53-59.

279. Sader HS, Castanheira M, Mendes RE, et al. Ceftazidimeavibactam activity against multidrug-resistant Pseudomonas aeruginosa isolated in U.S. medical centers in 2012 and 2013. Antimicrob Agents Chemother 2015;59:3656-3659.

280. Cubist Pharmaceuticals. Zerbaxa (ceftolozane/tazobactam): Full prescribing information. 2015; www.accessdata.fda. gov/drugsatfda_docs/label/2014/2068291bl.pdf. (Last accessed August 27, 2015).

281. Sykes RB, Bonner DP, Bush K, Georgopapadakou NH. Azthreonam (SQ 26,776), a synthetic monobactam specifically active against aerobic Gram-negative bacteria. Antimicrob Agents Chemother 1982;21:85-92.

282. McWilliams CS, Condon S, Schwartz RM, Ginocchio CC. Incidence of extended-spectrum- $\beta$ lactamase-producing Escherichia coli and Klebsiella pneumoniae isolates that test susceptible to cephalosporins and aztreonam by the revised CLSI breakpoints. J Clin Microbiol 2014;52: 2653-2655.
283. Terico AT, Gallagher JC. Beta-lactam hypersensitivity and cross-reactivity. J Pharm Pract 2014;27:530-544.

284. De Waele JJ, Tellado JM, Alder J, et al. Randomised clinical trial of moxifloxacin versus ertapenem in complicated intra-abdominal infections: Results of the PROMISE study. Int J Antimicrobial Agents 2013:41:57-64.

285. Schultsz C, Geerlings S. Plasmid-mediated resistance in Enterobacteriaceae: Changing landscape and implications for therapy. Drugs 2012;72:1-16.

286. Zhanel GG, Wiebe R, Dilay L, et al. Comparative review of the carbapenems. Drugs 2007;67:1027-1052.

287. Papp-Wallace KM, Endimiani A, Taracila MA, Bonomo RA. Carbapenems: Past, present, and future. Antimicrob Agents Chemother 2011;55:4943-4960.

288. Lucasti C, Jasovich A, Umeh O, et al. Efficacy and tolerability of IV doripenem versus meropenem in adults with complicated intra-abdominal infection: A phase III, prospective, multicenter, randomized, double-blind, noninferiority study. Clin Ther 2008;30:868-883.

289. Rice DA, Kaniga K, Lee M, Redman R. Activity of doripenem versus comparators in subjects with baseline bacteraemia in six pooled phase 3 clinical trials. Int $\mathbf{J}$ Antimicrob Agents 2013;41:388-392.

290. Cannavino CR, Castaneda-Ruiz B, Redman R, et al. Safety and tolerability of doripenem in hospitalized children with complicated intra-abdominal infection, complicated urinary tract infections and pneumonia. Pediatr Infect Dis J 2015;34:1264-1267.

291. Shionogi \& Co., Ltd. Doribax (doripenem): Full prescribing information. 2015; www.accessdata.fda.gov/ drugsatfda_docs/label/2015/022106s015lbl.pdf. (Last accessed September 7, 2015).

292. Verwaest C; Belgian Multicenter Study Group. Meropenem versus imipenem/cilastatin as empirical monotherapy for serious bacterial infections in the intensive care unit. Clin Microbiol Infect 2000;6:294-302.

293. Solomkin JS, Wilson SE, Christou NV, et al. Results of a clinical trial of clinafloxacin versus imipenem/cilastatin for intraabdominal infections. Ann Surg 2001;233:79-87.

294. Fomin P, Beuran M, Gradauskas A, et al. Tigecycline is efficacious in the treatment of complicated intraabdominal infections. Int J Surg 2005;3:35-47.

295. Oliva ME, Rekha A, Yellin A, et al. A multicenter trial of the efficacy and safety of tigecycline versus imipenem/ cilastatin in patients with complicated intra-abdominal infections. BMC Infect Dis 2005;5:88.

296. Chen Z, Wu J, Zhang, Y, et al. Efficacy and safety of tigecycline monotherapy vs. imipenem/cilastatin in Chinese patients with complicated intra-abdominal infections: A randomized controlled trial. BMC Infect Dis 2010; 10:217.

297. Brismar B, Malmborg AS, Tunevall G, et al. Piperacillintazobactam versus imipenem-cilastatin for treatment of intra-abdominal infections. Antimicrob Agents Chemother 1992;36:2766-2773.

298. Kiratisin P, Chongthaleong A, Tan TY, et al. Comparative in vitro activity of carbapenems against major Gramnegative pathogens: Results of Asia-Pacific surveillance from the COMPACT II study. Int J Antimicrob Agents 2012;39:311-316.

299. Mu YP, Liu RU, Wang LQ, et al. Moxifloxacin monotherapy for treatment of complicated intra-abdominal infections: A meta-analysis of randomised controlled trials. Int J Clin Pract 2012;66:210-217. 
300. Goldstein EJ, Solomkin JS, Citron DM, Alder JD. Clinical efficacy and correlation of clinical outcomes with in vitro susceptibility for anaerobic bacteria in patients with complicated intra-abdominal infections treated with moxifloxacin. Clin Infect Dis 2011;53:1074-1080.

301. Cohn SM, Lipsett PA, Buchman TG, et al. Comparison of intravenous/oral ciprofloxacin plus metronidazole versus piperacillin/tazobactam in the treatment of complicated intraabdominal infections. Ann Surg 2000;232:254-262.

302. Matthaiou DK, Peppas G, Bliziotis IA, Falagas ME. Ciprofloxacin/metronidazole versus $\beta$-lactam-based treatment of intra-abdominal infections: A meta-analysis of comparative trials. Int J Antimicrob Agents 2006;28:159-165.

303. Adefurin A, Sammons H, Jacqz-Aigrain E, Choonara I. Ciprofloxacin safety in paediatrics: A systematic review. Arch Dis Child 2011;96:874-880.

304. Bradley JS, Jackson MA; Committee on Infectious Diseases; American Academy of Pediatrics. The use of systemic and topical fluoroquinolones. Pediatrics 2011;128: e1034-e1045.

305. Bradford PA. Tigecycline: A first in class glycylcycline. Clin Microbiol Newsletter 2004;26:163-168.

306. Prasad P, Sun J, Danner RL, Natanson C. Excess deaths associated with tigecycline after approval based on noninferiority trials. Clin Infect Dis 2012;54:1699-1709.

307. Tasina E, Haidich AB, Kokkali S, Arvanitidou M. Efficacy and safety of tigecycline for the treatment of infectious diseases: A meta-analysis. Lancet Infect Dis 2011; 11:834-844

308. Yahav D, Lador A, Paul M, Leibovici L. Efficacy and safety of tigecycline: A systematic review and metaanalysis. J Antimicrob Chemother 2011;66:1963-1971.

309. Vardakas KZ, Rafailidis PI, Falagas ME. Effectiveness and safety of tigecycline: Focus on use for approved indications. Clin Infect Dis 2012;54:1672-1674.

310. McGovern PC, Wible M, El-Tahtawy A, et al. All-cause mortality imbalance in the tigecycline phase 3 and 4 clinical trials. Int J Antimicrob Agents 2013;41:463-467.

311. Denys GA, Callister SM, Dowzicky MJ. Antimicrobial susceptibility among gram-negative isolates collected in the USA between 2005 and 2011 as part of the Tigecycline Evaluation and Surveillance Trial (T.E.S.T.). Ann Clin Microbiol Antimicrob 2013;12:24.

312. Sader HS, Farrell DJ, Flamm RK, Jones RN. Variation in potency and spectrum of tigecycline activity against bacterial strains from U.S. medical centers since its approval for clinical use (2006 to 2012). Antimicrob Agents Chemother 2014;58:2274-2280.

313. Bassetti M, Nicolini L, Repetto E, et al. Tigecycline use in serious nosocomial infections: A drug use evaluation. BMC Infect Dis 2010;10:287.

314. Eckmann C, Montravers P, Bassetti M, et al. Efficacy of tigecycline for the treatment of complicated intraabdominal infections in real-life clinical practice from five European observational studies. J Antimicrob Chemother 2013;68(Suppl 2):ii25-ii35.

315. Maseda E, Denis SE, Riquelme A, Gilsanz F. Use of tigecycline in critically ill patients with serious nosocomial intra-abdominal infections. Rev Esp Quimioter 2013;26: 56-63.

316. Qureshi ZA, Paterson DL, Potoski BA, et al. Treatment outcome of bacteremia due to KPC-producing Klebsiella pneumoniae: Superiority of combination antimicrobial regimens. Antimicrob Agents Chemother 2012;56:2108-2113.
317. Tzouvelekis LS, Markogiannakis, Psichogiou M, et al. Carbapenemases in Klebsiella pneumoniae and other Enterobacteriaceae: An evolving crisis of global dimensions. Clin Microbiol Rev 2012;25:682-707.

318. Kmeid JG, Youssef MM, Kanafani ZA, Kanj SS. Combination therapy for Gram-negative bacteria: What is the evidence? Expert Rev Anti Infect Ther 2013;11:13551362.

319. Fraser JD, Aguayo P, Leys CM, et al. A complete course of intravenous antibiotics vs a combination of intravenous and oral antibiotics for perforated appendicitis in children: A prospective, randomized trial. J Pediatr Surg 2010; 45:1198-1202.

320. Rice HE, Brown RL, Gollin G, et al. Results of a pilot trial comparing prolonged intravenous antibiotics with sequential intravenous/oral antibiotics for children with perforated appendicitis. Arch Surg 2001;136:1391-1395.

321. Aldridge KE, O'Brien M. In vitro susceptibilities of the Bacteroides fragilis group species: Change in isolation rates significantly affects overall susceptibility data. J Clin Microbiol 2002;40:4349-4352.

322. Deshpande A, Pasupuleti V, Thota P, et al. Communityassociated Clostridium difficile infection and antibiotics: A meta-analysis. J Antimicrob Chemother 2013;68:1951-1961.

323. Owens RC Jr, Donskey CJ, Gaynes RP, et al. Antimicrobialassociated risk factors for Clostridium difficile infection. Clin Infect Dis 2008;46(Suppl 1):S19-S31.

324. Billington EO, Phang SH, Gregson DB, et al. Incidence, risk factors, and outcomes for Enterococcus spp. blood stream infections: A population-based study. Int J Infect Dis 2014;26:76-82.

325. Dahl A, Bruun NE. Enterococcus faecalis infective endocarditis: Focus on clinical aspects. Expert Rev Cardiovasc Ther 2013;11:1247-1257.

326. Fernández-Hidalgo N, Almirante $\mathrm{B}$, Gavaldà $\mathrm{J}$, et al. Ampicillin plus ceftriaxone is as effective as ampicillin plus gentamicin for treating Enterococcus faecalis infective endocarditis. Clin Infect Dis 2013;56:1261-1268.

327. Nadler EP, Reblock KK, Ford HR, Gaines BA. Monotherapy versus multi-drug therapy for the treatment of perforated appendicitis in children. Surg Infect (Larchmt) 2003;4:327-333.

328. Nadler EP, Gaines BA. The Surgical Infection Society guidelines on antimicrobial therapy for children with appendicitis. Surg Infect (Larchmt) 2008;9:75-83.

329. Biedenbach DJ, Bell JM, Sader HS, et al. Antimicrobial susceptibility of Gram-positive bacterial isolates from the Asia-Pacific region and an in vitro evaluation of the bactericidal activity of daptomycin, vancomycin, and teicoplanin: A SENTRY Program Report (2003-2004). Int J Antimicrob Agents 2007;30:143-149.

330. Chatterjee I, Iredell JR, Woods M, Lipman J. The implications of enterococci for the intensive care unit. Crit Care Resusc 2007;9:69-75.

331. McBride SJ, Upton A, Roberts, SA. Clinical characteristics and outcomes of patients with vancomycinsusceptible Enterococcus faecalis and Enterococcus faecium bacteraemia - a five-year retrospective review. Eur J Clin Microbiol Infect Dis 2010;29:107-114.

332. Conde-Estévez D, Grau S, Albanell J, et al. Clinical characteristics and outcomes of patients with vancomycinsusceptible Enterococcus faecalis and Enterococcus faecium bacteraemia in cancer patients. Eur J Clin Microbiol Infect Dis 2011;30:103-108. 
333. Sader HS, Watters AA, Fritsche TR, Jones RN. Daptomycin antimicrobial activity tested against methicillinresistant staphylococci and vancomycin-resistant enterococci isolated in European medical centers (2005). BMC Infect Dis 2007;7:29.

334. Bradley JS. Which antibiotic for resistant Gram-positives, and why? J Infect 2014;68(Suppl 1):S63-S75.

335. Cattoir V, Giard JC. Antibiotic resistance in Enterococcus faecium clinical isolates. Expert Rev Anti Infect Ther 2014;12:239-248.

336. Birmingham MC, Rayner CR, Meagher AK, et al. Linezolid for the treatment of multidrug-resistant, grampositive infections: Experience from a compassionate-use program. Clin Infect Dis 2003;36:159-168.

337. Len O, Montejo M, Cervera C, et al. Daptomycin is safe and effective for the treatment of gram-positive cocci infections in solid organ transplantation. Transpl Infect Dis 2014;16:532-538.

338. Balli EP, Venetis CA, Miyakis S. Systematic review and meta-analysis of linezolid versus daptomycin for treatment of vancomycin-resistant enterococcal bacteremia. Antimicrob Agents Chemother 2014;58:734-739.

339. Chuang YC, Wang JT, Lin HY, Chang SC. Daptomycin versus linezolid for treatment of vancomycin-resistant enterococcal bacteremia: Systematic review and metaanalysis. BMC Infect Dis 2014;14:687.

340. Fierobe L, Decré D, Mùller C, et al. Methicillin-resistant Staphylococcus aureus as a causative agent of postoperative intra-abdominal infection: Relation to nasal colonization. Clin Infect Dis 1999;29:1231-1238.

341. Solomkin JS. Antibiotic resistance in postoperative infections. Crit Care Med 2001;29(Suppl 4):N97-N99.

342. Kobayashi M, Mohri Y, Ohi M, et al. Risk factors for anastomotic leakage and favorable antimicrobial treatment as empirical therapy for intra-abdominal infection in patients undergoing colorectal surgery. Surg Today 2014; 44:487-493.

343. Mohr JF, Murray BE. Point: Vancomycin is not obsolete for the treatment of infection caused by methicillinresistant Staphylococcus aureus. Clin Infect Dis 2007: 44:1536-1542.

344. Liu C, Bayer A, Cosgrove SE, et al. Clinical practice guidelines by the Infectious Diseases Society of America for the treatment of methicillin-resistant Staphylococcus aureus infections in adults and children: Executive summary. Clin Infect Dis 2011;52:285-292.

345. Rivera AM, Boucher HW. Current concepts in antimicrobial therapy against select gram-positive organisms: Methicillin-resistant Staphylococcus aureus, penicillinresistant pneumococci, and vancomycin-resistant enterococci. Mayo Clin Proc 2011;86:1230-1243.

346. Solomkin JS, Bjornson HS, Cainzos M, et al. A consensus statement on empiric therapy for suspected gram-positive infections in surgical patients. Am J Surg 2004;187:134 145.

347. Deresinski S. Counterpoint: Vancomycin and Staphylococcus aureus-an antibiotic enters obsolescence. Clin Infect Dis 2007;44:1543-1548.

348. Pichereau S, Rose WE. Invasive community-associated MRSA infections: Epidemiology and antimicrobial management. Expert Opin Pharmacother 2010;11:3009-3025.

349. Holmes NE, Turnidge JD, Munckhof WJ, et al. Antibiotic choice may not explain poorer outcomes in patients with Staphylococcus aureus bacteremia and high vancomycin minimum inhibitory concentrations. J Infect Dis 2011; 204:340-347.

350. Gupta A, Biyani M, Khaira A. Vancomycin nephrotoxicity: Myths and facts. Neth J Med 2011;69:379-383.

351. Wong-Beringer A, Joo J, Tsed E, Beringer $P$. Vancomycin-associated nephrotoxicity: A critical appraisal of risk with high-dose therapy. Int $\mathrm{J}$ Antimicrob Agents 2011;37:95-101.

352. van Hal SJ, Paterson DL, Lodise TP. Systematic review and meta-analysis of vancomycin-induced nephrotoxicity associated with dosing schedules that maintain troughs between 15 and 20 milligrams per liter. Antimicrob Agents Chemother 2013;57:734-744.

353. Pletz MW, Burkhardt O, Welte T. Nosocomial methicillin-resistant Staphylococcus aureus (MRSA) pneumonia: Linezolid or vancomycin? - Comparison of pharmacology and clinical efficacy. Eur J Med Res 2010; 15:507-513.

354. McDaneld PM, Spooner LM, Mohr JF, Belliveau PP. Use of daptomycin to treat infections with methicillin-resistant Staphylococcus aureus isolates having vancomycin minimum inhibitory concentrations of 1.5 to $2 \mu \mathrm{g} / \mathrm{mL}$. Ann Pharmacother 2013;47:1654-1665.

355. Bassetti M, Baguneid M, Bouza E, et al. European perspective and update on the management of complicated skin and soft tissue infections due to methicillin-resistant Staphylococcus aureus after more than 10 years of experience with linezolid. Clin Microbiol Infect 2014;20(Suppl 4):3-18.

356. Dodds TJ, Hawke CI. Linezolid versus vancomycin for MRSA skin and soft tissue infections (systematic review and meta-analysis). ANZ J Surg 2009;79:629-635.

357. Bally M, Dendukuri N, Sinclair A, et al. A network metaanalysis of antibiotics for treatment of hospitalised patients with suspected or proven meticillin-resistant Staphylococcus aureus infection. Int $\mathbf{J}$ Antimicrob Agents 2012;40:479-495.

358. Rodvold KA, McConeghy KW. Methicillin-resistant Staphylococcus aureus therapy: Past, present, and future. Clin Infect Dis 2014;58(Suppl 1):S20-S27.

359. He W, Zhang Y, Chen H, et al. Efficacy and safety of daptomycin for the treatment of infectious disease: A meta-analysis based on randomized controlled trials. J Antimicrob Chemother 2014;69:3181-3189.

360. Hamill RJ. Amphotericin B formulations: A comparative review of efficacy and toxicity. Drugs 2013;73:919-934.

361. Mora-Duarte J, Betts R, Rotstein C, et al. Comparison of caspofungin and amphotericin B for invasive candidiasis. N Engl J Med 2002;347:2020-2029.

362. Kuse ER, Chetchotisakd P, da Cunha CA, et al. Micafungin versus liposomal amphotericin B for candidaemia and invasive candidosis: A phase III randomised doubleblind trial. Lancet 2007;369:1519-1527.

363. Abele-Horn M, Kopp A, Sternberg U, et al. A randomized study comparing fluconazole with amphotericin B/5flucytosine for the treatment of systemic Candida infections in intensive care patients. Infection 1996; 24:426432.

364. Gafter-Gvili A, Vidal L, Goldberg E, et al. Treatment of invasive candidal infections: Systematic review and metaanalysis. Mayo Clin Proc 2008;83:1011-1021.

365. Reboli AC, Rotstein C, Pappas PG, et al. Anidulafungin versus fluconazole for invasive candidiasis. N Engl J Med 2007;356:2472-2482. 
366. Eggimann P, Francioli P, Bille J, et al. Fluconazole prophylaxis prevents intra-abdominal candidiasis in high-risk surgical patients. Crit Care Med 1999;27:1066-1072.

367. Lass-Flörl C. Triazole antifungal agents in invasive fungal infections: A comparative review. Drugs 2011;71:24052419.

368. Fothergill AW, Sutton DA, McCarthy DI, Wiederhold NP. Impact of new antifungal breakpoints on antifungal resistance in Candida species. J Clin Microbiol 2014;52: 994-997.

369. Malani AN, Kerr LE, Kauffman CA. Voriconazole: How to use this antifungal agent and what to expect. Semin Respir Crit Care Med 2015;36:786-795.

370. Kullberg BJ, Sobel JD, Ruhnke M, et al. Voriconazole versus a regimen of amphotericin $\mathrm{B}$ followed by fluconazole for candidaemia in non-neutropenic patients: A randomised non-inferiority trial. Lancet 2005;366:1435-1442.

371. Queiroz-Telles F, Berezin E, Leverger G, et al. Micafungin versus liposomal amphotericin B for pediatric patients with invasive candidiasis: Substudy of a randomized double-blind trial. Pediatr Infect Dis J 2008;27:820-826.

372. Senn L, Eggimann P, Ksontini R, et al. Caspofungin for prevention of intra-abdominal candidiasis in high-risk surgical patients. Intensive Care Med 2009;35:903-908.

373. Kett DH, Shorr AF, Reboli AC, et al. Anidulafungin compared with fluconazole in severely ill patients with candidemia and other forms of invasive candidiasis: Support for the 2009 IDSA treatment guidelines for candidiasis. Crit Care 2011;15:R253.

374. Neoh CF, Slavin M, Chen SC, et al. Echinocandins in the treatment of candidaemia and invasive candidiasis: Clinical and economic perspectives. Int $\mathbf{J}$ Antimicrob Agents 2014;43:207-214.

375. Pappas PG, Kauffman CA, Andes D, et al. Clinical practice guidelines for the management of candidiasis: 2009 update by the Infectious Diseases Society of America. Clin Infect Dis 2009;48:503-535.

376. Solomkin JS, Reinhart HH, Dellinger EP, et al. Results of a randomized trial comparing sequential intravenous/oral treatment with ciprofloxacin plus metronidazole to imipenem/cilastatin for intra-abdominal infections. Ann Surg 1996;223:303-315.

377. Donahue PE, Smith DL, Yellin AE, et al. Trovafloxacin in the treatment of intra-abdominal infections: Results of a double-blind, multicenter comparison with imipenem/cilastatin. Am J Surg 1998;176(Suppl 6A):53S-61S.

378. Buck RE, Price KE. Cefadroxil, a new broad-spectrum cephalosporin. Antimicrob Agents Chemother 1977;11: 324-330.

379. Neu HC, Fu KP. Cefaclor: In vitro spectrum of activity and beta-lactamase stability. Antimicrob Agents Chemother 1978;13:584-588.

380. Wise R, Andrews JM, Ashby JP, Thornber D. The in-vitro activity of cefpodoxime: A comparison with other oral cephalosporins. J Antimicrob Chemother 1990;25:541-550.

381. Bauernfeind A, Jungwirth R. Antibacterial activity of cefpodoxime in comparison with cefixime, cefdinir, cefatamet, cefibuten, loracarbef, cefprozil, BAY 3522, cefuroxime, cefaclor, and cefadroxil. Infection 1991;19:353-362.

382. Thornsberry C. Review of the in vitro antibacterial activity of cefprozil, a new oral cephalosporin. Clin Infect Dis 1992;14(Suppl 2):S189-S194.

383. Schatz BS, Karavokiros KT, Taeubel MA, Itokazu GS. Comparison of cefprozil, cefpodoxime proxetil, lor- acarbef, cefixime, and ceftibuten. Ann Pharmacother 1996;30:258-268.

384. Scott LJ, Ormrod D, Goa KL. Cefuroxime axetil: An updated review of its use in the management of bacterial infections. Drugs 2001;61:1455-1500.

385. Sader HS, Jones RN. Cefdinir: An oral cephalosporin for the treatment of respiratory tract infections and skin and skin structure infections. Expert Rev Anti Infect Ther 2007;5:29-43.

386. Taylor E, Dev V, Shah D, et al. Complicated appendicitis: Is there a minimum intravenous antibiotic requirement? A prospective randomized trial. Am Surg 2000;66:887-890.

387. Gollin G, Abarbanell A, Moores D. Oral antibiotics in the management of perforated appendicitis in children. Am Surg 2002;68:1072-1074.

388. Adibe OO, Barnaby K, Dobies J, et al. Postoperative antibiotic therapy for children with perforated appendicitis: Long course of intravenous antibiotics versus early conversion to an oral regimen. Am J Surg 2008;195:141-143.

389. Merlino JI, Malangoni MA, Smith CM, Lange RL. Prospective randomized trials affect the outcomes of intraabdominal infection. Ann Surg 2001;233:859-866.

390. Wong PF, Gilliam AD, Kumar S, et al. Antibiotic regimens for secondary peritonitis of gastrointestinal origin in adults. Cochrane Database Syst Rev 2005;2:CD004539.

391. Brook I. Microbiology and management of intra-abdominal infections in children. Pediatr Int 2003;45:123-129.

392. Csendes A, Burdiles P, Maluenda F, et al. Simultaneous bacteriologic assessment of bile from gallbladder and common bile duct in control subjects and patients with gallstones and common duct stones. Arch Surg 1996; 131:389-394.

393. Guillet-Caruba C, Cheikhelard A, Guillet M, et al. Bacteriologic epidemiology and empirical treatment of pediatric complicated appendicitis. Diagn Microbiol Infect Dis 2011;69:376-381.

394. Schmitt F, Clermidi P, Dorsi M, et al. Bacterial studies of complicated appendicitis over a 20-year period and their impact on empirical antibiotic treatment. J Pediatr Surg 2012;47:2055-2062.

395. Society for Healthcare Epidemiology of America, Infectious Diseases Society of America, Pediatric Infectious Diseases Society. Policy statement on antimicrobial stewardship by the Society for Healthcare Epidemiology of America (SHEA), the Infectious Diseases Society of America (IDSA), and the Pediatric Infectious Diseases Society (PIDS). Infect Control Hosp Epidemiol 2012; 33:322-327.

396. Skrupky LP, Tellor BR, Mazuski JE. Current strategies for the treatment of complicated intraabdominal infections. Expert Opin Pharmacother 2013;14:1933-1947.

397. Hawser SP, Bouchillon SK, Hoban DJ, et al. Incidence and antimicrobial susceptibility of Escherichia coli and Klebsiella pneumoniae with extended-spectrum $\beta$-lactamases in community- and hospital-associated intra-abdominal infections in Europe: Results of the 2008 study for Monitoring Antimicrobial Resistance Trends (SMART). Antimicrob Agents Chemother 2010;54:3043-3046.

398. Anaya DA, Nathens AB. Risk factors for severe sepsis in secondary peritonitis. Surg Infect (Larchmt) 2003;4:355-362.

399. Fraser JD, Aguayo P, Sharp SW, et al. Physiologic predictors of postoperative abscess in children with perforated appendicitis: Subset analysis from a prospective randomized trial. Surgery 2010;147:729-732. 
400. Nataraja RM, Teague WJ, Galea J, et al. Comparison of intraabdominal abscess formation after laparoscopic and open appendicectomies in children. J Pediatr Surg 2012; 47:317-321.

401. Fallon SC, Brandt ML, Hassan SF, et al. Evaluating the effectiveness of a discharge protocol for children with advanced appendicitis. J Surg Res 2013;184:347351.

402. Frakking FN, Rottier WC, Dorigo-Zetsma JW, et al. Appropriateness of empirical treatment and outcome in bacteremia caused by extended-spectrum- $\beta$-lactamaseproducing bacteria. Antimicrob Agents Chemother 2013;57:3092-3099.

403. Theunissen C, Cherifi S, Karmali R. Management and outcome of high-risk peritonitis: A retrospective survey 2005-2009. Int J Infect Dis 2011;15:e769-e773.

404. Joo EJ, Kang CI, Ha YE, et al. Impact of inappropriate empiric antimicrobial therapy on outcome in Pseudomonas aeruginosa bacteraemia: A stratified analysis according to sites of infection. Infection 2011;39:309-318.

405. Brunkhorst FM, Oppert M, Marx G, et al. Effect of empirical treatment with moxifloxacin and meropenem vs meropenem on sepsis-related organ dysfunction in patients with severe sepsis: A randomized trial. JAMA 2012;307:2390-2399.

406. Hu Y, Li L, Li W, et al. Combination antibiotic therapy versus monotherapy for Pseudomonas aeruginosa bacteraemia: A meta-analysis of retrospective and prospective studies. Int J Antimicrob Agents 2013;42:492-496.

407. Cometta A, Baumgartner JD, Lew D, et al. Prospective randomized comparison of imipenem monotherapy with imipenem plus netilmicin for treatment of severe infections in nonneutropenic patients. Antimicrob Agents Chemother 1994;38:1309-1313.

408. Kaffarnik MF, Urban M, Hopt UT, Utzolino S. Impact of enterococcus on immunocompetent and immunosuppressed patients with perforation of the small or large bowel. Tech Health Care 2012;20:37-48.

409. Dowzicky MJ. Susceptibility to tigecycline and linezolid among gram-positive isolates collected in the United States as part of the Tigecycline Evaluation and Surveillance Trial (TEST) between 2004 and 2009. Clin Ther 2011;33:1964-1973.

410. Alvarez-Lerma F, Grau S, Ferrández O. Characteristics of doripenem: A new broad-spectrum antibiotic. Drug Des Devel Ther 2009;3:173-190.

411. Suppli M, Aabenhus R, Harboe ZB, et al. Mortality in enterococcal bloodstream infections increases with inappropriate antimicrobial therapy. Clin Microbiol Infect 2011;17:1078-1083.

412. Dupont H, Paugam-Burtz C, Muller-Serieys C, et al. Predictive factors of mortality due to polymicrobial peritonitis with Candida isolation in peritoneal fluid in critically ill patients. Arch Surg 2002;137:1341-1346.

413. Sandven P, Qvist H, Skovlund E, et al. Significance of Candida recovered from intraoperative specimens in patients with intra-abdominal perforations. Crit Care Med 2002;30:541-547.

414. Khoury W, Szold O, Soffer D, et al. Prophylactic fluconazole does not improve outcome in patients with purulent and fecal peritonitis due to lower gastrointestinal perforation. Am Surg 2010;76:197-202.

415. Blot SI, Vandewoudea KH, De Waele JJ. Candida peritonitis. Curr Opin Crit Care 2007;13:195-199.
416. Lee SC, Fung CP, Chen HY, et al. Candida peritonitis due to peptic ulcer perforation: Incidence rate, risk factors, prognosis and susceptibility to fluconazole and amphotericin B. Diagn Microbiol Infect Dis 2002;44:23-27.

417. de Ruiter J, Weel J, Manusama E, et al. The epidemiology of intra-abdominal flora in critically ill patients with secondary and tertiary abdominal sepsis. Infection 2009; 37:522-527.

418. Montravers P, Mira JP, Gangneux JP, et al. A multicentre study of antifungal strategies and outcome of Candida spp. peritonitis in intensive-care units. Clin Microbiol Infect 2011;17:1061-1067.

419. Borch JE, Andersen KE, Bindslev-Jensen C. The prevalence of suspected and challenge-verified penicillin allergy in a university hospital population. Basic Clin Pharmacol Toxicol 2006;98:357-362.

420. Wong BB, Keith PK, Waserman S. Clinical history as a predictor of penicillin skin test outcome. Ann Allergy Asthma Immunol 2006;97:169-174.

421. Sodhi M, Axtell SS, Callahan J, Shekar R. Is it safe to use carbapenems in patients with a history of allergy to penicillin? J Antimicrob Chemother 2004;54:1155-1157.

422. Prescott WA Jr, Kusmierski KA. Clinical importance of carbapenem hypersensitivity in patients with self-reported and documented penicillin allergy. Pharmacotherapy 2007;27:137-142.

423. Endimiani A, Paterson DL. Optimizing therapy for infections caused by Enterobacteriaceae producing extended-spectrum $\beta$-lactamases. Semin Respir Crit Care Med 2007;28:646-655.

424. Pitout JD. Infections with extended-spectrum $\beta$ lactamase-producing Enterobacteriaceae: Changing epidemiology and drug treatment choices. Drugs 2010;70: 313-333.

425. Chopra T, Marchaim D, Veltman J, et al. Impact of cefepime therapy on mortality among patients with bloodstream infections caused by extended-spectrum- $\beta$-lactamaseproducing Klebsiella pneumoniae and Escherichia coli. Antimicrob Agents Chemother 2012;56:3936-3942.

426. Huang CC, Chen YS, Toh HS, et al. Impact of revised CLSI breakpoints for susceptibility to third-generation cephalosporins and carbapenems among Enterobacteriaceae isolates in the Asia-Pacific region: Results from the Study for Monitoring Antimicrobial Resistance Trends (SMART), 2002-2010. Int J Antimicrob Agents 2012;40 (Suppl):S4-S10.

427. Lee NY, Lee CC, Huang WH, et al. Cefepime therapy for monomicrobial bacteremia caused by cefepime-susceptible extended-spectrum beta-lactamase-producing Enterobacteriaceae: MIC matters. Clin Infect Dis 2013;56:488-495.

428. Martinez-Martinez L, Hernandez-Alles S, Alberti S, et al. In vivo selection of porin-deficient mutants of Klebsiella pneumoniae with increased resistance to cefoxitin and expanded-spectrum cephalosporins. Antimicrob Agents Chemother 1996;40:342-348.

429. Nørskov-Lauritsen N, Marchandin H, Dowzicky MJ. Antimicrobial susceptibility of tigecycline and comparators against bacterial isolates collected as part of the TEST study in Europe (2004-2007). Int J Antimicrob Agents 2009;34:121-130.

430. Park YS, Adams-Haduch JM, Shutt KA, et al. Clinical and microbiologic characteristics of cephalosporin-resistant Escherichia coli at three centers in the United States. Antimicrob Agents Chemother 2012;56:1870-1876. 
431. Hawser SP, Badal RE, Bouchillon SK, et al. Monitoring the global in vitro activity of ertapenem against Escherichia coli from intra-abdominal infections: SMART 20022010. Int J Antimicrob Agents 2013;41:224-228.

432. Lynch JP III, Clark NM, Zhanel GG. Evolution of antimicrobial resistance among Enterobacteriaceae (focus on extended spectrum $\beta$-lactamases and carbapenemases). Expert Opin Pharmacother 2013;14:199-210.

433. Zimhony O, Chmelnitsky I, Bardenstein R, et al. Endocarditis caused by extended spectrum- $\beta$-lactamaseproducing Klebsiella pneumoniae: Emergence of resistance to ciprofloxacin and piperacillin-tazobactam during treatment despite initial susceptibility. Antimicrob Agents Chemother 2006;50:3179-3182.

434. Nordmann $P$, Naas T, Poirel L. Global spread of carbapenemase-producing Enterobacteriaceae. Emerg Infect Dis 2011;17:1791-1798.

435. Cantón R, Akóva M, Carmeli Y, et al. Rapid evolution and spread of carbapenemases among Enterobacteriaceae in Europe. Clin Microbiol Infect 2012;18:413-431.

436. Torer N, Yorganci K, Elker K, Sayek I. Prognostic factors of the mortality of postoperative intraabdominal infections. Infection 2010;38:255-260.

437. Chen YH, Hsueh PR. Changing bacteriology of abdominal and surgical sepsis. Curr Opin Infect Dis 2012;25:590-595.

438. Sartelli M, Viale P, Catena F, et al. 2013 WSES guidelines for management of intra-abdominal infections. World $\mathrm{J}$ Emerg Surg 2013;8:3.

439. Kang CI, Chung DR, Ko KS, et al. Clinical predictors for enterococcal bacteraemia in patients with bacteraemic intra-abdominal infections. Scand J Infect Dis 2010;42: 817-820.

440. Dupont H, Vael C, Muller-Serieys C, et al. Prospective evaluation of virulence factors of enterococci isolated from patients with peritonitis: Impact on outcome. Diagn Microbiol Infect Dis 2008;60:247-253.

441. Reik R, Tenover FC, Klein E, McDonald LC. The burden of vancomycin-resistant enterococcal infections in US hospitals, 2003 to 2004. Diagn Microbiol Infect Dis 2008;62:81-85.

442. Gilmore MS, Lebreton F, van Schaik W. Genomic transition of enterococci from gut commensals to leading causes of multidrug-resistant hospital infection in the antibiotic era. Curr Opin Microbiol 2013;16:10-16.

443. Ziakas PD, Pliakos EE, Zervou FN, et al. MRSA and VRE colonization in solid organ transplantation: A metaanalysis of published studies. Am J Transplant 2014;14: 1887-1894.

444. Reinert RR, Low DE, Rossi F, et al. Antimicrobial susceptibility among organisms from the Asia/Pacific Rim, Europe and Latin and North America collected as part of TEST and the in vitro activity of tigecycline. J Antimicrob Chemother 2007;60:1018-1029.

445. Deshpande LM, Fritsche TR, Moet GJ, et al. Antimicrobial resistance and molecular epidemiology of vancomycinresistant enterococci from North America and Europe: A report from the SENTRY antimicrobial surveillance program. Diag Microbiol Infect Dis 2007;58:163-170.

446. Florescu I, Beuran M, Dimov R, et al. Efficacy and safety of tigecycline compared with vancomycin or linezolid for treatment of serious infections with methicillin-resistant Staphylococcus aureus or vancomycin-resistant enterococci: A Phase 3, multicentre, double-blind, randomized study. J Antimicrob Chemother 2008;62(Suppl 1):i17-i28.
447. Heizmann WR, Löschmann PA, Eckmann C. Clinical efficacy of tigecycline used as monotherapy or in combination regimens for complicated infections with documented involvement of multiresistant bacteria. Infection 2015;43:37-43.

448. Nathens AB, Chu PT, Marshall JC. Nosocomial infection in the surgical intensive care unit. Infect Dis Clin North Am 1992;6:657-675.

449. Graham PL III, Lin SX, Larson EL. A U.S. populationbased survey of Staphylococcus aureus colonization. Ann Intern Med 2006;144:318-325.

450. Harbarth S, Sax H, Uckay I, et al. A predictive model for identifying surgical patients at risk of methicillin-resistant Staphylococcus aureus carriage on admission. J Am Coll Surg 2008;207:683-689.

451. McKinnell JA, Miller LG, Eells SJ, et al. A systematic literature review and meta-analysis of factors associated with methicillin-resistant Staphylococcus aureus colonization at time of hospital or intensive care unit admission. Infect Control Hosp Epidemiol 2013;34:1077-1086.

452. Rybak M, Lomaestro B, Rotschafer JC, et al. Therapeutic monitoring of vancomycin in adult patients: A consensus review of the American Society of Health-System Pharmacists, the Infectious Diseases Society of America, and the Society of Infectious Diseases Pharmacists. Am J Health-Syst Pharm 2009;66:82-98.

453. Denton M. Enterobacteriaceae. Int J Antimicrob Agents 2007;29(Suppl 3):S9-S22.

454. Augustin P, Tran-Dinh A, Valin N, et al. Pseudomonas aeruginosa post-operative peritonitis: Clinical features, risk factors, and prognosis. Surg Infect (Larchmt) 2013; 14:297-303.

455. Nordmann P, Cuzon G, Naas T. The real threat of Klebsiella pneumoniae carbapenemase-producing bacteria. Lancet Infect Dis 2009;9:228-236.

456. Zavascki AP, Carvalhaes CG, Picão RC, Gales AC. Multidrug-resistant Pseudomonas aeruginosa and Acinetobacter baumannii: Resistance mechanisms and implications for therapy. Expert Rev Anti Infect Ther 2010;8:71-93.

457. Cornaglia G, Giamarellou H, Rossolini GM. Metallo- $\beta$ lactamases: A last frontier for $\beta$-lactams? Lancet Infect Dis 2011;11:381-393.

458. Dalhoff A. Global fluoroquinolone resistance epidemiology and implictions for clinical use. Interdiscip Perspect Infect Dis 2012;2012:976273.

459. Liu YM, Chen YS, Toh HS, et al. In vitro susceptibilities of non-Enterobacteriaceae isolates from patients with intra-abdominal infections in the Asia-Pacific region from 2003 to 2010: Results from the Study for Monitoring Antimicrobial Resistance Trends (SMART). Int J Antimicrob Agents 2012;40 (Suppl 1):S11-S17.

460. Castanheira M, Deshpande LM, Costello A, et al. Epidemiology and carbapenem resistance mechanisms of carbapenem-non-susceptible Pseudomonas aeruginosa collected during 2009-11 in 14 European and Mediterranean countries. J Antimicrob Chemother 2014;69:1804-1814.

461. Namias N, Samiian L, Nino D, et al. Incidence and susceptibility of pathogenic bacteria vary between intensive care units within a single hospital: Implications for empiric antibiotic strategies. J Trauma 2000;49:638-645.

462. Binkley S, Fishman NO, LaRosa LA, et al. Comparison of unit-specific and hospital-wide antibiograms: Potential implications for selection of empirical antimicrobial therapy. Infect Control Hosp Epidemiol 2006;27:682-687. 
463. Kuster SP, Ruef C, Zbinden R, et al. Stratification of cumulative antibiograms in hospitals for hospital unit, specimen type, isolate sequence and duration of hospital stay. J Antimicrob Chemother 2008;62:1451-1461.

464. Paterson DL. Recommendation for treatment of severe infections caused by Enterobacteriaceae producing extended-spectrum $\beta$-lactamases (ESBLs). Clin Microbiol Infect 2000:6:460-463.

465. Endimiani A, Luzzaro F, Perilli M, et al. Bacteremia due to Klebsiella pneumoniae isolates producing the TEM-52 extended-spectrum $\beta$-lactamase: Treatment outcome of patients receiving imipenem or ciprofloxacin. Clin Infect Dis 2004;38:243-251.

466. Paterson DL, Ko WC, Von Gottberg A, et al. Antibiotic therapy for Klebsiella pneumoniae bacteremia: Implications of production of extended-spectrum $\beta$-lactamases. Clin Infect Dis 2004;39:31-37.

467. Nguyen HM, Shier KL, Graber CJ. Determining a clinical framework for use of cefepime and $\beta$-lactam $/ \beta$-lactamase inhibitors in the treatment of infections caused by extendedspectrum- $\beta$-lactamase-producing Enterobacteriaceae. J Antimicrob Chemother 2014;69:871-880.

468. Hilty M, Sendi P, Seiffert SN, et al. Characterisation and clinical features of Enterobacter cloacae bloodstream infections occurring at a tertiary care university hospital in Switzerland: Is cefepime adequate therapy? Int J Antimicrob Agents 2013;41:236-249.

469. Castanheira M, Mills JC, Costello SE, et al. Ceftazidimeavibactam activity tested against Enterobacteriaceae isolates from U.S. hospitals (2011 to 2013) and characterization of $\beta$-lactamase-producing strains. Antimicrob Agents Chemother 2015;59:3509-3517.

470. Yoshizumi A, Ishii Y, Aoki K, et al. In vitro susceptibility of characterized $\beta$-lactamase-producing Gram-negative bacteria isolated in Japan to ceftazidime-, ceftaroline-, and aztreonam-avibactam combinations. J Infect Chemother 2015;21:148-151.

471. Tumbarello M, Viale P, Viscoli C, et al. Predictors of mortality in bloodstream infections caused by Klebsiella pneumoniae carbapenemase-producing K. pneumoniae: Importance of combination therapy. Clin Infect Dis 2012;55:943-950.

472. Falagas ME, Lourida P, Poulikakos P, et al. Antibiotic treatment of infections due to carbapenem-resistant Enterobacteriaceae: Systematic evaluation of the available evidence. Antimicrob Agents Chemother 2014;58:654-663.

473. Lee CR, Lee JH, Park KS, et al. Global dissemination of carbapenemase-producing Klebsiella pneumoniae: Epidemiology, genetic context, treatment options, and detection methods. Front Microbiol 2016;7:895.

474. Magiorakos AP, Srinivasan A, Carey RB, et al. Multidrugresistant, extensively drug-resistant and pandrug-resistant bacteria: An international expert proposal for interim standard definitions for acquired resistance. Clin Microbiol Infect 2012;18:268-281.

475. Maseda E, Mensa J, Valía JC, et al. Bugs, hosts and ICU environment: Countering pan-resistance in nosocomial microbiota and treating bacterial infections in the critical care setting. Rev Esp Quimioter 2013;26:312-331.

476. Giamarellou H, Poulakou G. Multidrug-resistant Gramnegative infections: What are the treatment options? Drugs 2009;69:1879-1901.

477. Biswas S, Brunel JM, Dubus JC, et al. Colistin: An update on the antibiotic of the 21st century. Expert Rev Anti Infect Ther 2012;10:917-934.
478. Dalfino L, Puntillo F, Mosca A, et al. High-dose, extended-interval colistin administration in critically ill patients: Is this the right dosing strategy? A preliminary study. Clin Infect Dis 2012;54:1720-1726.

479. Vicari G, Bauer SR, Neuner EA, Lam SW. Association between colistin dose and microbiologic outcomes in patients with multidrug-resistant gram-negative bacteremia. Clin Infect Dis 2013;56:398-404.

480. Karaiskos I, Giamarellou H. Multidrug-resistant and extensively drug-resistant Gram-negative pathogens: Current and emerging therapeutic approaches. Expert Opin Pharmacother 2014;15:1351-1370.

481. Kassamali Z, Jain R, Danziger LH. An update on the arsenal for multidrug-resistant Acinetobacter infections: Polymyxin antibiotics. Int J Infect Dis 2015;30:125-132.

482. Blumberg HM, Jarvis WR, Soucie JM, et al. Risk factors for candidal bloodstream infections in surgical intensive care unit patients: The NEMIS prospective multicenter study. Clin Infect Dis 2001;33:177-186.

483. Leleu G, Aegerter P, Guidet B; Collège des Utilisateurs de Base de Données en Réanimation. Systemic candidiasis in intensive care units: A multicenter, matched-cohort study. J Crit Care 2002;17:168-175.

484. Puzniak L, Teutsch S, Powderly W, Polish L. Has the epidemiology of nosocomial candidemia changed? Infect Control Hosp Epidemiol 2004;25:628-633.

485. Paphitou NI, Ostrosky-Zeichner L, Rex JH. Rules for identifying patients at increased risk for candidal infections in the surgical intensive care unit: Approach to developing practical criteria for systematic use in antifungal prophylaxis trials. Med Mycol 2005;43:235-243.

486. León C, Ruiz-Santana S, Saavedra P, et al. A bedside scoring system ("Candida score") for early antifungal treatment in nonneutropenic critically ill patients with Candida colonization. Crit Care Med 2006;34:730-737.

487. Ostrosky-Zeichner L, Sable C, Sobel J, et al. Multicenter retrospective development and validation of a clinical prediction rule for nosocomial invasive candidiasis in the intensive care setting. Eur J Clin Microbiol Infect Dis 2007;26:271-276.

488. Leroy O, Gangneux JP, Montravers P, et al. Epidemiology, management, and risk factors for death of invasive Candida infections in critical care: A multicenter, prospective, observational study in France (2005-2006). Crit Care Med 2009;37:1612-1618.

489. Carneiro HA, Mavrakis A, Mylonakis E. Candida peritonitis: An update on the latest research and treatments. World J Surg 2011;35:2650-2659.

490. Calandra T, Bille J, Schneider R, et al. Clinical significance of Candida isolated from peritoneum in surgical patients. Lancet 1989;2:1437-1440.

491. Hsu FC, Lin PC, Chi CY, et al. Prognostic factors for patients with culture-positive Candida infection undergoing abdominal surgery. J Microbiol Immunol Infect 2009;42:378-384.

492. Solomkin JS, Flohr AB, Quie PG, Simmons RL. The role of Candida in intraperitoneal infections. Surgery 1980;88: 524-530.

493. Pittet D, Monod M, Suter PM, et al. Candida colonization and subsequent infections in critically ill surgical patients. Ann Surg 1994;220:751-758.

494. Sandven P, Giercksky KE; NORGAS Group, and the Norwegian Yeast Study Group. Yeast colonization in surgical patients with intra-abdominal perforations. Eur J Clin Microbiol Infect Dis 2001;20:475-481. 
495. Hoerauf A, Hammer S, Müller-Myhsok B, Rupprecht H. Intra-abdominal Candida infection during acute necrotizing pancreatitis has a high prevalence and is associated with increased mortality. Crit Care Med 1998;26:2010-2015.

496. Vege SS, Gardner TB, Chari ST, et al. Outcomes of intraabdominal fungal vs. bacterial infections in severe acute pancreatitis. Am J Gastroenterol 2009;104:2065-2070.

497. Blot SI, Vandewoude KH, Hoste EA, Colardyn FA. Effects of nosocomial candidemia on outcomes of critically ill patients. Am J Med 2002;113:480-485.

498. Morrell M, Fraser VJ, Kollef MH. Delaying the empiric treatment of candida bloodstream infection until positive blood culture results are obtained: A potential risk factor for hospital mortality. Antimicrob Agents Chemother 2005;49:3640-3645.

499. Shan YS, Sy ED, Wang ST, et al. Early presumptive therapy with fluconazole for occult Candida infection after gastrointestinal surgery. World J Surg 2006;30:119-126.

500. Hsu DI, Nguyen M, Nguyen L, et al. A multicentre study to evaluate the impact of timing of caspofungin administration on outcomes of invasive candidiasis in non-immunocompromised adult patients. J Antimicrob Chemother 2010;65:1765-1770.

501. Kollef M, Micek S, Hampton N, et al. Septic shock attributed to Candida infection: Importance of empiric therapy and source control. Clin Infect Dis 2012;54:1739-1746.

502. Bassetti M, Marchetti M, Chakrabarti A, et al. A research agenda on the management of intra-abdominal candidiasis: Results from a consensus of multinational experts. Intensive Care Med 2013;39:2092-2106.

503. Pea F. Current pharmacological concepts for wise use of echinocandins in the treatment of Candida infections in septic critically ill patients. Expert Rev Ant Infect Ther 2013;11:989-997.

504. Pfaller MA, Andes DR, Diekema DJ, et al. Epidemiology and outcomes of invasive candidiasis due to non-albicans species of Candida in 2,496 patients: Data from the Prospective Antifungal Therapy (PATH) registry 2004-2008. PLoS One 2014;9:e101510.

505. Pfaller MA, Diekema DJ. Progress in antifungal susceptibility testing of Candida spp. by use of Clinical and Laboratory Standards Institute broth microdilution methods, 2010 to 2012. J Clin Microbiol 2012;50:2846-2856.

506. Barie PS, Hydo LJ, Shou J, et al. Influence of antibiotic therapy on mortality of critical surgical illness caused or complicated by infection. Surg Infect (Larchmt) 2005; 6:41-54.

507. Kumar A, Roberts D, Wood KE, et al. Duration of hypotension before initiation of effective antimicrobial therapy is the critical determinant of survival in human septic shock. Crit Care Med 2006;34:1589-1596.

508. Gaieski DF, Mikkelsen ME, Band RA, et al. Impact of time to antibiotics on survival in patients with severe sepsis or septic shock in whom early goal-directed therapy was initiated in the emergency department. Crit Care Med 2010;38:1045-1053.

509. Corona A, Bertolini G, Lipman J, et al. Antibiotic use and impact on outcome from bacteraemic critical illness: The BActeraemia Study in Intensive Care (BASIC). J Antimicrob Chemother 2010;65:1276-1285.

510. Puskarich MA, Trzeciak S, Shapiro NI, et al. Association between timing of antibiotic administration and mortality from septic shock in patients treated with a quantitative resuscitation protocol. Crit Care Med 2011;39:2066-2071.
511. Vilella AL, Seifert CF. Timing and appropriateness of initial antibiotic therapy in newly presenting septic patients. Am J Emerg Med 2014;32:7-13.

512. Ryoo SM, Kim WY, Sohn CH, et al. Prognostic value of timing of antibiotic administration in patients with septic shock treated with early quantitative resuscitation. Am J Med Sci 2015;349:328-333.

513. Mangram AJ, Horan TC, Pearson ML, et al. Guideline for prevention of surgical site infection, 1999. Infect Cont Hosp Epidemiol 1999;20:250-278.

514. Engelman R, Shahian D, Shemin R, et al. The Society of Thoracic Surgeons Practice Guideline Series: Antibiotic prophylaxis in cardiac surgery, part II: Antibiotic choice. Ann Thorac Surg 2007;83:1569-1576.

515. Anderson DJ, Kaye KS, Classen D, et al. Strategies to prevent surgical site infections in acute care hospitals. Infect Cont Hosp Epidemiol 2008;29 (Suppl 1):S51-S61.

516. Classen DC, Evans RS, Persotnik SL, et al. The timing of prophylactic administration of antibiotics and the risk of surgical-wound infection. N Engl J Med 1992;326:281-286.

517. Garey KW, Dao T, Chen H, et al. Timing of vancomycin prophylaxis for cardiac surgery patients and the risk of surgical site infections. J Antimicrob Chemother 2006;58: 645-650.

518. van Kasteren ME, Manniën J, Ott A, et al. Antibiotic prophylaxis and the risk of surgical site infections following total hip arthroplasty: Timely administration is the most important factor. Clin Infect Dis 2007;44:921-927.

519. Weber WP, Marti WR, Zwahlen M, et al. The timing of surgical antimicrobial prophylaxis. Ann Surg 2008; 247:918-926.

520. Steinberg JP, Braun BI, Hellinger WC, et al. Timing of antimicrobial prophylaxis and the risk of surgical site infections: Results from the Trial to Reduce Antimicrobial Prophylaxis Errors. Ann Surg 2009;250:10-16.

521. Zanetti G, Giardina R, Platt R. Intraoperative redosing of cefazolin and risk for surgical site infection in cardiac surgery. Emerg Infect Dis 2001;7:828-831.

522. Morita S, Nishisho I, Nomura T, et al. The significance of the intraoperative repeated dosing of antimicrobials for preventing surgical wound infection in colorectal surgery. Surg Today 2005;35:732-738.

523. United States Food and Drug Administration. Drugs@ FDA. FDA approved drug products. www.accessdata.fda .gov/scripts/cder/drugsatfda/index.cfm. (Last accessed November 19, 2015).

524. Brunton LL, Chabner BA, Knollmann BC (eds). Goodman and Gilman's The Pharmacological Basis of Therapeutics, 12th ed. New York: McGraw-Hill Medical, 2011.

525. Mueller EW, Boucher BA. The use of extended-interval aminoglycoside dosing strategies for the treatment of moderate-to-severe infections encountered in critically ill surgical patients. Surg Infect (Larchmt) 2009;10:563-570.

526. Pea F, Viale P. Bench-to-bedside review: Appropriate antibiotic therapy in severe sepsis and septic shock-does the dose matter? Crit Care 2009;13:214.

527. Eyler RF, Mueller BA. Antibiotic dosing in critically ill patients with acute kidney injury. Nat Rev Nephrol 2011; 7:226-235.

528. Roberts JA, Joynt GM, Choi GY, et al. How to optimise antimicrobial prescriptions in the Intensive Care Unit: Principles of individualised dosing using pharmacokinetics and pharmacodynamics. Int $\mathrm{J}$ Antimicrob Agents 2012;39:187-192. 
529. Luyt CE, Bréchot N, Trouillet JL, Chastre J. Antibiotic stewardship in the intensive care unit. Crit Care 2014; 18:480.

530. Ho VP, Jenkins SG, Afaneh CI, et al. Use of meropenem by continuous infusion to treat a patient with a Bla(kpc-2)positive Klebsiella pneumoniae blood stream infection. Surg Infect 2011;12:325-327.

531. Taccone FS, Cotton F, Roisin S, et al. Optimal meropenem concentrations to treat multidrug-resistant Pseudomonas aeruginosa septic shock. Antimicrob Agents Chemother 2012;56:2129-2131.

532. Taccone FS, Laterre PF, Dugernier T, et al. Insufficient $\beta$ lactam concentrations in the early phase of severe sepsis and septic shock. Crit Care 2010;14:R126.

533. Cockcroft DW, Gault MH. Prediction of creatinine clearance from serum creatinine. Nephron 1976;16:31-41.

534. Patel N, Scheetz MH, Drusano GL, Lodise TP. Determination of antibiotic dosage adjustments in patients with renal impairment: Elements for success. J Antimicrob Chemother 2010;65:2285-2290.

535. Brown DL, Masselink AJ, Lalla CD. Functional range of creatinine clearance for renal drug dosing: A practical solution to the controversy of which weight to use in the Cockcroft-Gault equation. Ann Pharmacother 2013;47: 1039-1044.

536. Fissell WH. Antimicrobial dosing in acute renal replacement. Adv Chronic Kidney Dis 2013;20:85-93.

537. Carlier M, Dumoulin A, Janssen A, et al. Comparison of different equations to assess glomerular filtration in critically ill patients. Intensive Care Med 2015;41:427-435.

538. Choi G, Gomersall CD, Tian Q, et al. Principles of antibacterial dosing in continuous renal replacement therapy. Crit Care Med 2009;37:2268-2282.

539. Gilbert B, Robbins P, Livornese LL Jr. Use of antibacterial agents in renal failure. Infect Dis Clin North Am 2009;23:899-924.

540. Heintz BH, Matzke GR, Dager WE. Antimicrobial dosing concepts and recommendations for critically ill adult patients receiving continuous renal replacement therapy or intermittent hemodialysis. Pharmacotherapy 2009;29: $562-577$.

541. Ulldemolins M, Roberts JA, Lipman J, Rello J. Antibiotic dosing in multiple organ dysfunction syndrome. Chest 2011;139:1210-1220.

542. Halilovic J, Heintz BH. Antibiotic dosing in cirrhosis. Am Health Syst Pharm 2014;71:1621-1634.

543. Bearden DT, Rodvold KA. Dosage adjustments for antibacterials in obese patients: Applying clinical pharmacokinetics. Clin Pharmacokinet 2000;38:415-426.

544. Erstad BL. Dosing of medications in morbidly obese patients in the intensive care unit setting. Intensive Care Med 2004;30:18-32.

545. Pai MP, Bearden DT. Antimicrobial dosing considerations in obese adult patients. Pharmacotherapy 2007;27:1081-1091.

546. Hanley MJ, Abernethy DR, Greenblatt DJ. Effect of obesity on the pharmacokinetics of drugs in humans. Clin Pharamacokinet 2010;49:71-87.

547. Jain R, Chung SM, Khurana M, et al. Implications of obesity for drug therapy: Limitations and challenges. Clin Pharmacol Ther 2011;90:77-89.

548. Janson B, Thursky K. Dosing of antibiotics in obesity. Curr Opin Infect Dis 2012;25:634-649.

549. Verhave JC, Fesler P, Ribstein J, et al. Estimation of renal function in subjects with normal serum creatinine levels:
Influence of age and body mass index. Am J Kidney Dis 2005;46:233-241.

550. Salazar DE, Corcoran GB. Predicting creatinine clearance and renal drug clearance in obese patients from estimated fat-free body mass. Am J Med 1988;84:1053-1060.

551. Snider RD, Kruse JA, Bander JJ, Dunn GH. Accuracy of estimated creatinine clearance in obese patients with stable renal function in the intensive care unit. Pharmacotherapy 1995;15:747-753.

552. Bauer LA, Blouin RA, Griffen WO Jr, et al. Amikacin pharmacokinetics in morbidly obese patients. Am J Hosp Pharm 1980;37:519-522.

553. Bauer LA, Edwards WAD, Dellinger EP, Simonowitz DA. Influence of weight on aminoglycoside pharmacokinetics in normal weight and morbidly obese patients. Eur J Clin Pharmacol 1983;24:643-647.

554. Leader WG, Tsubaki T, Chandler MH. Creatinineclearance estimates for predicting gentamicin pharmacokinetic values in obese patients. Am J Hosp Pharm 1994;51:2125-2130.

555. Traynor AM, Nafziger AN, Bertino JS Jr. Aminoglycoside dosing weight correction factors for patients of various body sizes. Antimicrob Agents Chemother 1995;39:545-548.

556. Payne KD, Hall RG II. Dosing of antibacterial agents in obese adults: Does one size fit all? Expert Rev Anti Infect Ther 2014;12:829-854.

557. Yuk J, Nightingale CH, Sweeney K, et al. Pharmacokinetics of nafcillin in obesity. J Infect Dis 1988;157:10881089.

558. Chen M, Nafziger AN, Drusano GL, et al. Comparative pharmacokinetics and pharmacodynamic target attainment of ertapenem in normal-weight, obese, and extremely obese adults. Antimicrob Agents Chemother 2006;50:1222-1227.

559. Newman D, Scheetz MH, Adeyemi OA, et al. Serum piperacillin/tazobactam pharmacokinetics in a morbidly obese individual. Ann Pharmacother 2007;41:1734-1739.

560. Barbour A, Schmidt S, Rout WR, et al. Soft tissue penetration of cefuroxime determined by clinical microdialysis in morbidly obese patients undergoing abdominal surgery. Int J Antimicrob Agents 2009;34:231-235.

561. Toma O, Suntrup P, Stefanescu A, et al. Pharmacokinetics and tissue penetration of cefoxitin in obesity: Implications for risk of surgical site infection. Anesth Analg 2011;113: 730-737.

562. van Kralingen S, Taks M, Diepstraten J, et al. Pharmacokinetics and protein binding of cefazolin in morbidly obese patients. Eur J Clin Pharmacol 2011;67:985-992.

563. Roberts JA, Lipman J. Optimal doripenem dosing simulations in critically ill nosocomial pneumonia patients with obesity, augmented renal clearance, and decreased bacterial susceptibility. Crit Care Med 2013;41:489-495.

564. Caldwell JB, Nilsen AK. Intravenous ciprofloxacin dosing in a morbidly obese patient. Ann Pharmacother 1994;28: 806.

565. Hollenstein UM, Brunner M, Schmid R, Müller M. Soft tissue concentrations of ciprofloxacin in obese and lean subjects following weight-adjusted dosing. Int $\mathrm{J}$ Obes Relat Metab Disord 2001;25:354-358.

566. Utrup TR, Mueller EW, Healy DP, et al. High-dose ciprofloxacin for serious gram-negative infection in an obese, critically ill patient receiving continuous venovenous hemodiafiltration. Ann Pharmacother 2010;44:1660-1664.

567. Cook AM, Martin C, Adams VR, Morehead RS. Pharmacokinetics of intravenous levofloxacin administered at 
750 milligrams in obese adults. Antimicrob Agents Chemother 2011;55:3240-3243.

568. Kees MG, Weber S, Kees F, Horbach T. Pharmacokinetics of moxifloxacin in plasma and tissue of morbidly obese patients. J Antimicrob Chemother 2011;66:2330-2335.

569. United Kingdom Clinical Pharmacy Association. Drug dosing in extremes of body weight in critically ill patients, 1st ed. 2013; www.scottishintensivecare.org.uk/uploads/ 2014-07-24-19-55-33-Drugdosingatextremesofbod-45662 .pdf. (Last accessed July 15, 2015).

570. Stein GE, Schooley SL, Peloquin CA, et al. Pharmacokinetics and pharmacodynamics of linezolid in obese patients with cellulitis. Ann Pharmacother 2005;39:427-432.

571. Puzniak LA, Morrow LE, Huang DB, Barreto JN. Impact of weight on treatment efficacy and safety in complicated skin and skin structure infections and nosocomial pneumonia caused by methicillin-resistant Staphylococcus aureus. Clin Ther 2013;35:1557-1570.

572. Dvorchik BH, Damphousse D. The pharmacokinetics of daptomycin in moderately obese, morbidly obese, and matched nonobese subjects. J Clin Pharmacol 2005;45: 48-56.

573. Pai MP, Norenberg JP, Anderson T, et al. Influence of morbid obesity on the single-dose pharmacokinetics of daptomycin. Antimicrob Agents Chemother 2007;51: 2741-2747.

574. Zakrison TL, Hille DA, Namias N. Effect of body mass index on treatment of complicated intra-abdominal infections in hospitalized adults: Comparison of ertapenem with piperacillin-tazobactam. Surg Infect 2012;13:38-42.

575. Dietch ZC, Duane TM, Cook CH, et al. Obesity is not associated with antimicrobial treatment failure for intraabdominal infection. Surg Infect (Larchmt) 2016;17:412421.

576. Roberts JA, Lipman J. Antibacterial dosing in intensive care. Pharmacokinetics, degree of disease and pharmacodynamics of sepsis. Clin Pharmacokinet 2006;45:755-773.

577. McKinnon PS, Paladino JA, Schentag JJ. Evaluation of area under the inhibitory curve (AUIC) and time above the minimum inhibitory concentration $(\mathrm{T}>\mathrm{MIC})$ as predictors of outcome for cefepime and ceftazidime in serious bacterial infections. Int J Antimicrob Agents 2008;31:345-351.

578. Roberts JA, Lipman J, Blot S, Rello J. Better outcomes through continuous infusion of time-dependent antibiotics to critically ill patients? Curr Opin Crit Care 2008;14: 390-396.

579. Falagas ME, Tansarli GS, Ikawa K, Vardakas KZ. Clinical outcomes with extended or continuous versus short-term intravenous infusion of carbapenems and piperacillin/tazobactam: A systematic review and meta-analysis. Clin Infect Dis 2013;56:272-82.

580. Shiu J, Wang E, Tejani AM, Wasdell M. Continuous versus intermittent infusions of antibiotics for the treatment of severe acute infections. Cochrane Database Syst Rev 2013:3;CD008481.

581. Seguin P, Verdier MC, Chanavaz C, et al. Plasma and peritoneal concentration following continuous infusion of cefotaxime in patients with secondary peritonitis. J Antimicrob Chemother 2009;63:564-567.

582. Roberts JA, Webb S, Paterson D, et al. A systematic review on clinical benefits of continuous administration of $\beta$-lactam antibiotics. Crit Care Med 2009;37:2071-2078.

583. Dulhunty JM, Roberts JA, Davis JS, et al. A multicenter randomized trial of continuous versus intermittent $\beta$ - lactam infusion in severe sepsis. Am J Respir Crit Care Med 2015;192:1298-1305.

584. Lau WK, Mercer D, Itani KM, et al. Randomized, openlabel, comparative study of piperacillin-tazobactam administered by continuous infusion versus intermittent infusion for treatment of hospitalized patients with complicated intraabdominal infection. Antimicrob Agents Chemother 2006; 50:3556-3561.

585. Li C, Kuti JL, Nightingale $\mathrm{CH}$, et al. Population pharmacokinetics and pharmacodynamics of piperacillin/tazobactam in patients with complicated intra-abdominal infection. J Antimicrob Chemother 2005;56:388-395.

586. Rice LB. The Maxwell Finland Lecture: For the duration-Rational antibiotic administration in an era of antimicrobial resistance and Clostridium difficile. Clin Infect Dis 2008;46:491-496.

587. Nordback I, Sand J, Saaristo R, Paajanen H. Early treatment with antibiotics reduces the need for surgery in acute necrotizing pancreatitis-a single-center randomized study. J Gastrointest Surg 2001;5:113-118.

588. Isenmann R, Rünzi M, Kron M, et al. Prophylactic antibiotic treatment in patients with predicted severe acute pancreatitis: A placebo-controlled, double-blind trial. Gastroenterology 2004;126:997-1004.

589. Dellinger EP, Tellado JM, Soto NE, et al. Early antibiotic treatment for severe acute necrotizing pancreatitis: A randomized, double-blind, placebo-controlled study. Ann Surg 2007;245:674-683.

590. Røkke O, Harbitz TB, Liljedal J, et al. Early treatment of severe pancreatitis with imipenem: A prospective randomized clinical trial. Scand J Gastroenterol 2007;42:771-776.

591. Xue P, Deng LH, Zhang ZD, et al. Effect of antibiotic prophylaxis on acute necrotizing pancreatitis: Results of a randomized controlled trial. J Gastroenterol Hepatol 2009;24:736-742.

592. García-Barrasa A, Borobia FG, Pallares R, et al. A doubleblind, placebo-controlled trial of ciprofloxacin prophylaxis in patients with acute necrotizing pancreatitis. J Gastrointest Surg 2009;13:768-774.

593. Mazaki T, Ishii Y, Takayama T. Meta-analysis of prophylactic antibiotic use in acute necrotizing pancreatitis. Br J Surg 2006;93:674-684.

594. Bai Y, Gao J, Zou DW, Li ZS. Prophylactic antibiotics cannot reduce infected pancreatic necrosis and mortality in acute necrotizing pancreatitis: Evidence from a metaanalysis of randomized controlled trials. Am J Gastroenterol 2008;103:104-110.

595. Jafri NS, Mahid SS, Idstein SR, et al. Antibiotic prophylaxis is not protective in severe acute pancreatitis: A systematic review and meta-analysis. Am J Surg 2009; 197:806-813.

596. Villatoro E, Mulla M, Larvin M. Antibiotic therapy for prophylaxis against infection of pancreatic necrosis in acute pancreatitis. Cochrane Database Syst Rev 2010;5: CD002941.

597. Wittau M, Mayer B, Scheele J, et al. Systematic review and meta-analysis of antibiotic prophylaxis in severe acute pancreatitis. Scand J Gastroenterol 2011;46:261-270.

598. Jiang K, Huang W, Yang XN, Xia Q. Present and future of prophylactic antibiotics for severe acute pancreatitis. World J Gastroenterol 2012;18:279-284.

599. Lim CL, Lee W, Liew YX, et al. Role of antibiotic prophylaxis in necrotizing pancreatitis: A meta-analysis. J Gastrointest Surg 2015;19:480-491. 
600. Dambrauskas Z, Gulbinas A, Pundzius J, Barauskas G. Meta-analysis of prophylactic parenteral antibiotic use in acute necrotizing pancreatitis. Medicina (Kaunas) 2007; 43:291-300.

601. Xu T, Cai Q. Prophylactic antibiotic treatment in acute necrotizing pancreatitis: Results from a meta-analysis. Scand J Gastroenterol 2008;43:1249-1258.

602. Hart PA, Bechtold ML, Marshall JB, et al. Prophylactic antibiotics in necrotizing pancreatitis: A meta-analysis. South Med J 2008;101:1126-1131.

603. Yao L, Huang X, Li Y, et al. Prophylactic antibiotics reduce pancreatic necrosis in acute necrotizing pancreatitis: A meta-analysis of randomized trials. Dig Surg 2010;27:442-449.

604. de Vries AC, Besselink MGH, Buskens E, et al. Randomized controlled trials of antibiotic prophylaxis in severe acute pancreatitis: Relationship between methodological quality and outcome. Pancreatology 2007;7:531-538.

605. Hjern F, Josephson T, Altman D, et al. Conservative treatment of acute colonic diverticulitis: Are antibiotics always mandatory? Scand J Gastroenterol 2007;42:41-47.

606. de Korte N, Kuyvenhoven JP, van der Peet DL, et al. Mild colonic diverticulitis can be treated without antibiotics. A case-control study. Colorectal Dis 2012;14:325-330.

607. Chabok A, Pahlman L, Hjern F, et al. Randomized clinical trial of antibiotics in acute uncomplicated diverticulitis. $\mathrm{Br}$ J Surg 2012;99:532-539.

608. Shabanzadeh DM, Wille-Jorgensen P. Antibiotics for uncomplicated diverticulitis. Cochrane Database Systematic Rev 2012;11:CD009092.

609. McDonald M, Grabsch E, Marshall C, Forbes A. Singleversus multiple-dose antimicrobial prophylaxis for major surgery: A systematic review. Aust N Z J Surg 1998;68: 388-396.

610. Fonseca SN, Kunzle SR, Junqueira MJ, et al. Implementing 1-dose antibiotic prophylaxis for prevention of surgical site infection. Arch Surg 2006;141:1109-1114.

611. Fabian TC, Croce MA, Payne LW, et al. Duration of antibiotic therapy for penetrating abdominal trauma: A prospective trial. Surgery 1992;112:788-794.

612. Bozorgzadeh A, Pizzi WF, Barie PS, et al. The duration of antibiotic administration in penetrating abdominal trauma. Am J Surg 1999;177:125-131.

613. Kirton OC, O’Neill PA, Kestner M, Tortella BJ. Perioperative antibiotic use in high-risk penetrating hollow viscus injury: A prospective randomized, double-blind, placebo-control trial of 24 hours versus 5 days. J Trauma 2000;49:822-832.

614. Mui LM, Ng CS, Wong SK, et al. Optimum duration of prophylactic antibiotics in acute non-perforated appendicitis. ANZ J Surg 2005;75:425-428.

615. Regimbeau JM, Fuks D, Pautrat K, et al. Effect of postoperative antibiotic administration on postoperative infection following cholecystectomy for acute calculous cholecystitis: A randomized clinical trial. JAMA 2014; 312:145-154.

616. Andåker L, Höjer H, Kihlström E, Lindhagen J. Stratified duration of prophylactic antimicrobial treatment in emergency abdominal surgery. Metronidazole-fosfomycin vs. metronidazole-gentamicin in 381 patients. Acta Chir Scand 1987;153:185-192.

617. Schein M, Assalia A, Bachus H. Minimal antibiotic therapy after emergency abdominal surgery: A prospective study. Br J Surg 1994;81:989-991.
618. Gleisner AL, Argenta R, Pimentel M, et al. Infective complications according to duration of antibiotic treatment in acute abdomen. Int J Infect Dis 2004;8:155-162.

619. Hughes MJ, Harrison E, Paterson-Brown S. Post-operative antibiotics after appendectomy and post-operative abscess development: A retrospective analysis. Surg Infect (Larchmt) 2013;14:56-61.

620. van Rossem CC, Schreinemacher MH, Treskes K, et al. Duration of antibiotic treatment after appendicectomy for acute complicated appendicitis. Br J Surg 2014;101:715-719.

621. Hedrick TL, Evans HL, Smith RL, et al. Can we define the ideal duration of antibiotic therapy? Surg Infect (Larchmt) 2006; 7:419-432.

622. Riccio LM, Popovsky KA, Hranjec T, et al. Association of excessive duration of antibiotic therapy for intraabdominal infection with subsequent extra-abdominal infection and death: a study of 2,552 consecutive infections. Surg Infect (Larchmt) 2014;15:41-424.

623. Basoli A, Chirletti P, Cirino E, et al. A prospective, doubleblind, multicenter, randomized trial comparing ertapenem 3 vs $\geq 5$ days in community-acquired intraabdominal infection. J Gastrointest Surg 2008;12:592-600.

624. Lennard ES, Minshew BH, Dellinger EP, Wertz M. Leukocytosis at termination of antibiotic therapy: Its importance for intra-abdominal sepsis. Arch Surg 1980;115: 918-921.

625. Lennard ES, Dellinger EP, Wertz MJ, Minshew BH. Implications of leukocytosis and fever at conclusion of antibiotic therapy for intra-abdominal sepsis. Ann Surg 1982;195:19-24.

626. Daneman N, Shore K, Pinto R, Fowler R. Antibiotic treatment duration for bloodstream infections in critically ill patients: A national survey of Canadian infectious diseases and critical care specialists. Int $\mathbf{J}$ Antimicrob Agents 2011;38:480-485.

627. Havey TC, Fowler RA, Pinto R, et al. Duration of antibiotic therapy for critically ill patients with bloodstream infections: A retrospective cohort study. Can J Infect Dis Med Microbiol 2013;24:129-137.

628. Havey TC, Fowler RA, Daneman N. Duration of antibiotic therapy for bacteremia: A systematic review and meta-analysis. Crit Care 2011;15:R267.

629. Visser MR, Bosscha K, Olsman J, et al. Predictors of recurrence of fulminant bacterial peritonitis after discontinuation of antibiotics in open management of the abdomen. Eur J Surg 1998;164:825-829.

630. Dougherty SH. Antimicrobial culture and susceptibility testing has little value for routine management of secondary bacterial peritonitis. Clin Infect Dis 1997;25 (Suppl 2):S258-S261.

631. Leone M, Bourgoin A, Cambon S, et al. Empirical antimicrobial therapy of septic shock patients: Adequacy and impact on the outcome. Crit Care Med 2003;31:462-467.

632. Morel J, Casoetto J, Jospé R, et al. De-escalation as part of a global strategy of empiric antibiotherapy management. A retrospective study in a medico-surgical intensive care unit. Crit Care 2010;14:R225.

633. Montravers P, Dupont H, Gauzit R, et al. Strategies of initiation and streamlining of antibiotic therapy in 41 French intensive care units. Crit Care 2011;15:R17.

634. Dubrovskaya Y, Papadopoulos J, Scipione MR, et al. Antibiotic stewardship for intra-abdominal infections: Early impact on antimicrobial use and patient outcomes. Infect Cont Hosp Epidemiol 2012;33:427-429. 
635. Heenen S, Jacobs F, Vincent JL. Antibiotic strategies in severe nosocomial sepsis: Why do we not de-escalate more often? Crit Care Med 2012;40:1404-1409.

636. Gonzalez L, Cravoisy A, Barraud D, et al. Factors influencing the implementation of antibiotic de-escalation and impact of this strategy in critically ill patients. Crit Care 2013; 17:R140.

637. Paugam-Burtz C, Dupont H, Marmuse JP, et al. Daily organ-system failure for diagnosis of persistent intraabdominal sepsis after postoperative peritonitis. Intensive Care Med 2002;28:594-598.

638. Novotny AR, Emmanuel K, Hueser N, et al. Procalcitonin ratio indicates successful surgical treatment of abdominal sepsis. Surgery 2009;145:20-26.

639. Barie PS, Williams MD, McCollam JS, et al. Benefit/risk profile of drotrecogin alfa (activated) in surgical patients with severe sepsis. Am J Surg 2004;188:212-220.

640. Sirinek KR. Diagnosis and treatment of intra-abdominal abscesses. Surg Infect (Larchmt) 2000;1:31-38.

641. Lee MJ. Non-traumatic abdominal emergencies: Imaging and intervention in sepsis. Eur Radiol 2002;12:2172-2179.

642. Meeson S, Alvey CM, Golding SJ. Justifying multidetector CT in abdominal sepsis: Time for review? Br J Radiol 2009;82:190-197.

643. Go HL, Baarslag HJ, Vermeulen H, et al. A comparative study to validate the use of ultrasonography and computed tomography in patients with post-operative intra-abdominal sepsis. Eur J Radiol 2005;54:383-387.

644. Lin CM, Hung GU, Chao TH, et al. The limited use of ultrasound in the detection of abdominal abscesses in patients after colorectal surgery: Compared with gallium scan and computed tomography. Hepatogastroenterology 2005;52:79-81.

645. Bader FG, Schröder M, Kujath P, et al. Diffuse postoperative peritonitis - value of diagnostic parameters and impact of early indication for relaparotomy. Eur $\mathrm{J}$ Med Res 2009;14:491-496.

646. Allen BC, Barnhart H, Bashir M, et al. Diagnostic accuracy of intra-abdominal fluid collection characterization in the era of multidetector computed tomography. Am Surg 2012;78:185-189.

647. Gnannt R, Fischer MA, Baechler T, et al. Distinguishing infected from noninfected abdominal fluid collections after surgery: An imaging, clinical, and laboratory-based scoring system. Invest Radiol 2015;50:17-23.

648. Nielsen JW, Kurtovic KJ, Kenney BD, Diefenbach KA. Postoperative timing of computed tomography scans for abscess in pediatric appendicitis. J Surg Res 2016;200:1-7.

649. Sarkissian H, Hyman N, Osler T. Postoperative fluid collections after colon resection: The utility of clinical assessment. Am J Surg 2013;206:551-554.

650. McDermott FD, Heeney A, Kelly ME, et al. Systematic review of preoperative, intraoperative and postoperative risk factors for colorectal anastomotic leaks. Br J Surg 2015;102:462-479.

651. Antevil JL, Egan JC, Woodbury RO, et al. Abdominal computed tomography for postoperative abscess: Is it useful during the first week? J Gastrointest Surg 2006; 10:901-905.

652. van Santvoort HC, Besselink MG, Bakker OJ, et al. A step-up approach or open necrosectomy for necrotizing pancreatitis. N Engl J Med 2010;362:1491-1502.

653. Rangel-Frausto MS, Pittet D, Costigan M, et al. The natural history of the systemic inflammatory response syndrome (SIRS). A prospective study. JAMA 1995; 273:117-123.

654. Niven DJ, Léger C, Stelfox HT, Laupland KB. Fever in the critically ill: A review of epidemiology, immunology, and management. J Intensive Care Med 2012; 27:290-297.

655. Bouadma L, Luyt CE, Tubach F, et al. Use of procalcitonin to reduce patients' exposure to antibiotics in intensive care units (PRORATA trial): A multicentre randomised controlled trial. Lancet 2010;375:463-474.

656. Maseda E, Suarez-de-la-Rica A, Anillo V, et al. Procalcitonin-guided therapy may reduce length of antibiotic treatment in intensive care unit patients with secondary peritonitis: A multicenter retrospective study. J Crit Care 2015;30:537-542.

657. Negm AA, Poos H, Kruck E, et al. Microbiologic analysis of peri-pancreatic fluid collected during EUS in patients with pancreatitis: Impact on antibiotic therapy. Gastrointest Endosc 2013;78:303-311.

658. van Baal MC, Bollen TL, Bakker OJ, et al. The role of routine fine-needle aspiration in the diagnosis of infected necrotizing pancreatitis. Surgery 2014;155:442-448.

659. Hadley GP. Intra-abdominal sepsis—epidemiology, aetiology and management. Semin Pediatr Surg 2014;23:357-362.

660. Andersen BR, Kallehave FL, Andersen HK. Antibiotics versus placebo for prevention of postoperative infection after appendicectomy. Cochrane Database Syst Rev 2005;3:CD001439.

661. Lee SL, Islam S, Cassidy LD, et al. Antibiotics and appendicitis in the pediatric population: an American Pediatric Surgical Association Outcomes and Clinical Trials Committee systematic review. J Pediatr Surg 2010;45:2181-2185.

662. Sirinek KR, Levine BA. A randomized trial of ticarcillin and clavulanate versus gentamicin and clindamycin in patients with complicated appendicitis. Surg Gynecol Obstet 1991;172 (Suppl):30-35.

663. Dougherty SH, Sirinek KR, Schauer PR, et al. Ticarcillin/ clavulanate compared with clindamycin/gentamicin (with or without ampicillin) for the treatment of intra-abdominal infections in pediatric and adult patients. Am Surg 1995;61:297-303.

664. Uhari M, Seppänen J, Heikkinen E. Imipenem-cilastatin vs. tobramycin and metronidazole for appendicitis-related infections. Pediatr Infect Dis J 1992;11:445-450.

665. Allo MD, Bennion RS, Kathir K, et al. Ticarcillin/clavulanate versus imipenem/cilistatin for the treatment of infections associated with gangrenous and perforated appendicitis. Am Surg 1999;65:99-104.

666. Bradley JS, Faulkner KL, Klaugman KP. Efficacy, safety and tolerability of meropenem as empiric antibiotic therapy in hospitalized pediatric patients. Pediatr Infect Dis J 1996;15:749-757.

667. Schropp KP, Kaplan S, Golladay ES, et al. A randomized clinical trial of ampicillin, gentamicin and clindamycin versus cefotaxime and clindamycin in children with ruptured appendicitis. Surg Gynecol Obstet 1991;172:351-356.

668. Ciftci AO, Tanyel FC, Büyükpamukçu N, Hiçsönmez A. Comparative trial of four antibiotic combinations for perforated appendicitis in children. Eur J Surg 1997;163:591-596.

669. Adderson EE, Flynn PM, Hoffman JM. Efficacy and safety of cefepime in pediatric patients: A systematic review and meta-analysis. J Pediatr 2010;157:490-495.

670. Stutman HR. Clinical experience with aztreonam for treatment of infections in children. Rev Infect Dis 1991; 13(Suppl 7):S582-S585. 
671. Burkhardt JE, Hill MA, Carlton WW, Kesterson JW. Histologic and histochemical changes in articular cartilages of immature beagle dogs dosed with difloxacin, a fluoroquinolone. Vet Pathol 1990;27:162-170.

672. Stahlmann R, Kühner S, Shakibaei M, et al. Chondrotoxicity of ciprofloxacin in immature beagle dogs: Immunohistochemistry, electron microscopy and drug plasma concentrations. Arch Toxicol 2000;73:564-572.

673. Bradley JS, Behrendt CE, Arrieta AC, et al. Convalescent phase outpatient parenteral antiinfective therapy for children with complicated appendicitis. Pediatr Infect Dis J 2001;20:19-24.

674. Newman N, Wattad E, Greenberg D, et al. Communityacquired complicated intra-abdominal infections in children hospitalized during 1995-2004 at a paediatric surgery department. Scand J Infect Dis 2009;41:720-726.

675. Meller JL, Reyes HM, Loeff DS, et al. One-drug versus two-drug antibiotic therapy in pediatric perforated appendicitis: A prospective randomized study. Surgery 1991;110:764-767.

676. Emil S, Taylor M, Ndiforchu F, Nguyen N. What are the true advantages of a pediatric appendicitis clinical pathway? Am Surg 2006;72:885-889.

677. Henry MC, Walker A, Silverman BL, et al. Risk factors for the development of abdominal abscess following operation for perforated appendicitis in children: A multicenter case-control study. Arch Surg 2007;142:236-241.

678. Shawyer AC, Hatchell AC, Pemberton J, Flageole H. Compliance with published recommendations for postoperative antibiotic management of children with appendicitis: A chart audit. J Pediatr Surg 2015;50:783-785.

679. Dobremez E, Lavrand F, Lefevre Y, et al. Treatment of post-appendectomy intra-abdominal deep abscesses. Eur J Pediatr Surg 2003;13:393-397.

680. Gasior AC, Marty Knott E, Ostlie DJ, St. Peter SD. To drain or not to drain: An analysis of abscess drains in the treatment of appendicitis with abscess. Pediatr Surg Int 2013;29:455-458.

681. Rao SC, Basani L, Simmer K, et al. Peritoneal drainage versus laparotomy as initial surgical treatment for perforated necrotizing enterocolitis or spontaneous intestinal perforation in preterm low birth weight infants. Cochrane Database Syst Rev 2011;6:CD006182.
682. Moss RL, Dimmitt RA, Barnhart DC, et al. Laparotomy versus peritoneal drainage for necrotizing enterocolitis and perforation. N Engl J Med 2006;354:2225-2234.

683. Rees CM, Eaton S, Kiely EM, et al. Peritoneal drainage or laparotomy for neonatal bowel perforation? A randomized controlled trial. Ann Surg 2008;248:44-51.

684. Tickell D, Duke T. Evidence behind the WHO guidelines: Hospital care for children: For young infants with suspected necrotizing enterocolitis (NEC), what is the effectiveness of different parenteral antibiotic regimens in preventing progression and sequelae? J Trop Pediatr 2010;56:373-378.

685. Brook I. Microbiology and management of neonatal necrotizing enterocolitis. Am J Perinatol 2008;25:111-118.

686. Thompson AM, Bizzarro MJ. Necrotizing enterocolitis in newborns. Pathogenesis, prevention and management. Drugs 2008;68:1227-1238.

687. Shah D, Sinn JK. Antibiotic regimens for the empirical treatment of newborn infants with necrotising enterocolitis. Cochrane Database Syst Rev 2012;8:CD007448.

688. Zani A, Eaton S, Puri P, et al. International survey on the management of necrotizing enterocolitis. Eur J Pediatr Surg 2015;25:27-33.

689. Solomkin JS, Ramesh MK, Cesnauskas G, et al. Phase 2, randomized, double-blind study of the efficacy and safety of two dose regimens of eravacycline versus ertapenem for adult community-acquired complicated intraabdominal infections. Antimicrob Agents Chemother 2014;58:1847-1854.

690. Lucasti C, Vasile L, Sandesc D, et al. Phase 2, doseranging study of relebactam with imipenem-cilastatin in subjects with complicated intra-abdominal infection. Antimicrob Agents Chemother 2016;60:6234-6243.

Address correspondence to: Dr. John E. Mazuski Department of Surgery Washington University School of Medicine Campus Box 8109 660 S. Euclid Avenue Saint Louis, MO 63110-1093

E-mail:mazuskij@wustl.edu 